NISTIR 7575

\title{
Proceedings of the Dynamic Measurement and Control for Autonomous Manufacturing Workshop
}

\author{
Edited by: \\ Tsai Hong \\ Roger Eastman \\ Roger Bostelman \\ Hui-Min Huang \\ U.S DEPARTMENT OF COMMERCE \\ National Institute of Standards and Technology \\ Intelligent Systems Division \\ Gaithersburg, MD 20899-8230
}

and

Brian McNorris

SICK, Inc.

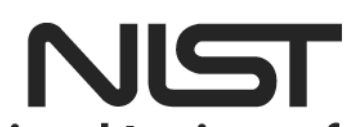

National Institute of Standards and Technology

U.S. Department of Commerce 
NISTIR 7575

\title{
Proceedings of the Dynamic Measurement and Control for Autonomous Manufacturing Workshop
}

\author{
Edited by: \\ Tsai Hong \\ Roger Eastman \\ Roger Bostelman \\ Hui-Min Huang \\ U.S DEPARTMENT OF COMMERCE \\ Technology Administration \\ National Institute of Standards and Technology \\ Intelligent Systems Division \\ Gaithersburg, MD 20899-8230
}

and

Brian McNorris

SICK, Inc

May 2009

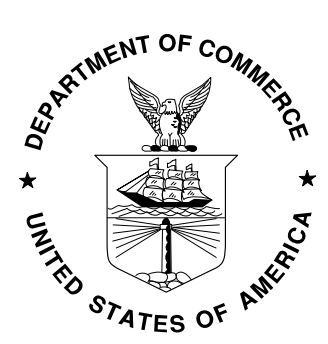

U.S. DEPARTMENT OF COMMERCE

Gary Locke, Secretary 



\section{PROCEEDINGS OF THE DYNAMIC MEASUREMENT AND CONTROL FOR AUTONOMOUS MANUFACTURING WORKSHOP}

Edited by:

Tsai Hong, Roger Eastman, Roger Bostelman, Hui-Min Huang

National Institute of Standards and Technology (NIST)

Brian McMorris

SICK, Inc.

Dates:

October $10-11,2007$

Location:

Loyola College of Maryland

Columbia, Maryland

Sponsors:

NIST, Loyola College in Maryland, and SICK, Inc. 


\section{TABLE OF CONTENTS}

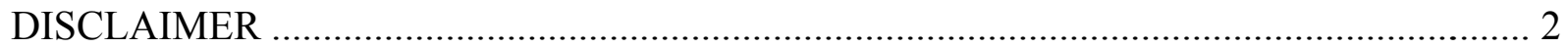

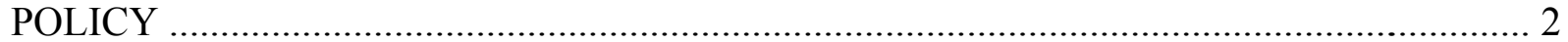

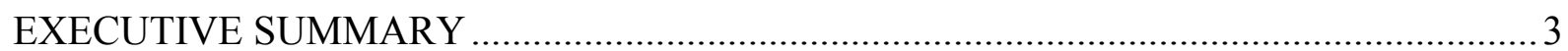

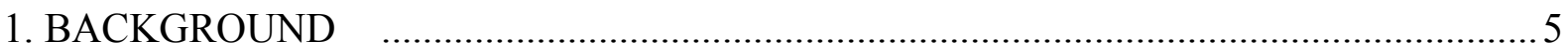

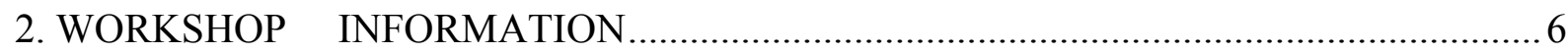

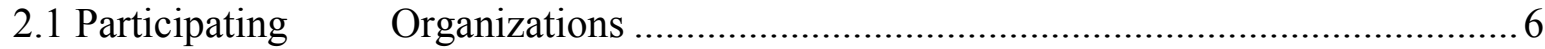

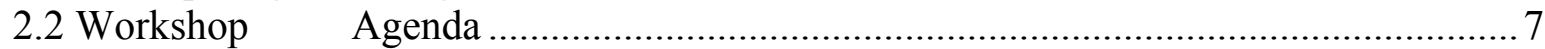

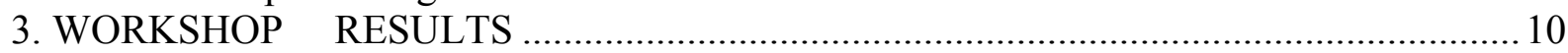

3.1 Day 1 Breakout Sessions Outcome .................................................................. 10

A. $\quad$ Requirements for dynamic 6DOF metrology for automated general assembly ....10

B. $\quad$ Control and perception needs for automated guided vehicles (AGVs) .................13

C. Information needs for real-time process monitoring and control ..........................15

D. Automated manipulators, their subcomponents and controls needed for dynamic

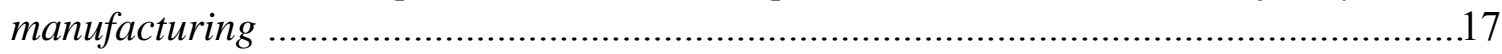

3.2 Day 2 Wrap-up Discussion and Results ...................................................20

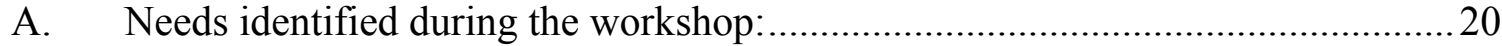

B. Action Items and Associated Volunteers........................................................ 20

4. INITIAL ACTION ITEMS RESULTS ................................................................ 22

4.1 Formulating New Projects for Identified Critical Needs ..................................22

4.2 Further Investigation on Industrial Needs ....................................................22

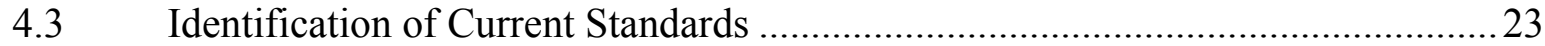

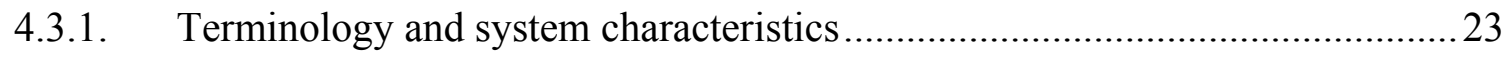

4.3.2. Safety and performance ...................................................................... 24

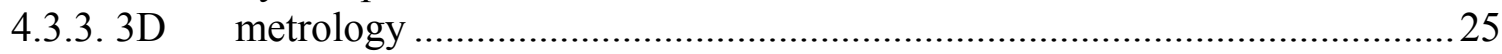

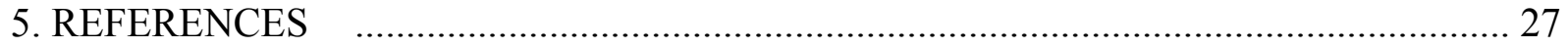

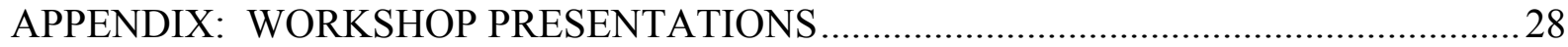




\section{DISCLAIMER}

Certain trade names and company products are mentioned in the text or identified in certain illustrations. When permissions for publication were provided to National Institute of Standards and Technology (NIST), the presentations of the participating companies or products are included in the Appendix to facilitate communications among the participants. In no case does such an identification imply recommendation or endorsement by the NIST, nor does it imply that the products are necessarily the best available for the purpose.

The opinions expressed in this Workshop Report are those of the workshop participants' and are not the official opinions of NIST.

\section{POLICY}

It is NIST's policy to us e the International System of Units (SI). However, som e of the units used in the workshop presentations and papers are in U.S. custom ary units because of the intended audience. Conversions from the U. S. customary units to SI have been $\mathrm{m}$ ade where possible. 


\section{EXECUTIVE SUMMARY}

The Dynamic Measurement and Control for Autonomous Manufacturing workshop was held on October 10 - 11, 2007 at the Columbia Graduate Center of Loyola College in Maryland. Forty-eight people attended the one and one-half day event which was moderated by Roger Bostelman and Tsai Hong of the National Institute of Standards and Technology (NIST) with assistance from Roger Eastman of Loyola and cosponsored by Brian McMorris of SICK, Inc. Attendees included current and potential users of autonomous manufacturing equipment, manufacturers of general assembly equipment, robotic arms and automated guided vehicles, and of machine vision and three-dimensional (3D) sensors, system integrators, government representatives from NIST and National Science Foundation (NSF), and academics with research specialties in computer vision and robotics.

The workshop was a part of an effort by NIST Manufacturing Engineering Laboratory (MEL) to assist industry in articulating the general requirements for advanced automation in manufacturing. The stated goal of the workshop was:

"To collect community input on requirements for the operation of next generation manufacturing robots, automated guided vehicles (AGVs), and intelligent assist devices in dynamic, changing environments. Specific topics to be considered are perception needs for dynamic visual servoing in autonomous assembly and requirements for the safe and effective operation of robot arms and AGVs in dynamic environments."

Current robot and AGV installations are generally rigid, sensor-poor and expensive to reconfigure. Standards, metrics, and measures are required to assist general assembly manufacturers in introducing new technologies and deploying flexible, sensor-rich systems suitable for dynamic operating environments. These new systems could enable equipment reuse, fewer dedicated installations, and faster and more flexible plant reconfiguration, resulting in significant cost savings and higher productivity. Industry, academia, and government research institute input and collaboration will enable NIST to articulate requirements and to explore methodologies to assist the manufacturers in evaluating and validating new technologies.

The first morning of the workshop consisted of a series of presentations given by manufacturers highlighting their needs, by academics on the state of current research in relevant areas, and by sensor vendors on the relevant capabilities. Also presented was the status of ASTM E57 3D Imaging System standards development efforts. In the afternoon, the attendees participated in four breakout sessions on particular challenges before reassembling to share the results. The focuses of the break out groups are

A. dynamic metrology and perception on the assembly line,

B. needs for enhanced robot control systems to operate in dynamic environments,

C. the requirements for advanced AGV use in dynamic environments, and

D. real-time sensing to validate and update virtual simulations. 
Most of these topics originally arose in the Fall 2006 Smart Assembly Workshop held at NIST. Workshop discussions continued the next morning in a plenary setting to summarize and to develop action items. The results are summarized below:

1. Perception systems for automation in dynamic environments must be comprehensive, pervasive and redundant. In scenarios such as a robot grasping a moving part off an assembly line, a single, narrowly focused sensor will not be sufficient. A single sensor may fail, may not be robust enough for the task, may not sense other objects that could become obstacles, may not be able to adequately sense humans in the workspace to prevent accidents, may have the wrong wavelength or modality to be useful in a task, or may have a fixed resolution while the task requires sensing at multiple resolutions. Perception for such scenarios will require sensor fusion and control logic to facilitate arbitration between multiple subsystems. Sensory modeling must be improved so that the performance can be compared and evaluated.

2. While most participants agreed on the general nature and need for next generation robots and perception systems, the group wanted to see more specifically defined and challenging scenarios to focus future discussions and to direct future research, much as the DARPA Grand Challenge does for mobile robotics. This issue was established as a follow-up action item for this group. Related to this issue, common themes in the breakout session discussions were the need for terminology standards, high level robot control vocabularies; common interfaces that support operations in dynamic environments, and articulation of requirements to identify standard useful tasks.

3. The need for performance standards and measurement techniques for localization was echoed by multiple breakout session groups. Dynamic metrology is required to provide reference measurements so that the performance of the perception systems can be evaluated for safety, for AGV navigation, and for moving part manipulation in changing environments. To judge whether an AGV or a robot arm can perform a task which involves motion with respect to a second moving object, we need techniques for calibrating and measuring absolute and relative motions.

4. Moving to next generation robotics with safe and reliable performance in dynamic environments will require the development of an entire cycle of commercial interests that can produce the components to be integrated into next generation assembly lines. As such, system integrators and standard specifications are needed for the purposes of component interfacing and test and evaluation. This concept and technology, in its entirety, is young and evolving, therefore, may take time to mature. Also hindering the progress is a chicken-andegg problem with new technology, because few companies can afford to invest in research or the application of a technology until the technology has been demonstrated as successful. To alleviate the dilemma, participants proposed to use testbeds and high fidelity simulation systems so that the technology can be validated before commercialization should begin.

5. Software is an important element of the perception systems for the control the motion of robots, AGVs and other automation devices. Capabilities are required to validate the software to ensure safety and to achieve reliable performance. 


\section{BACKGROUND}

In most manufacturing assembly and material transport environments, parts are delivered on moving lines to be picked up and attached to the base assemblies, which are usually also moving. Currently, an automated facility must be equipped with expensive, custom designed mechanisms, such as specific bowl feeders and conveyances and fixtures to control the motion and the positioning of the parts. Systems that are customized for certain current needs are typically not scalable enough to handle next generation manufacturing that features unstructured and dynamic environment. Such a new environment requires systems that are real-time controllable, agile, adaptable, flexible, and reconfigurable. Also required are nextgeneration safety technologies that advance human-robot collaboration to a new level.

New technologies, including advanced perception and advanced planning and motion control are required to achieve this next generation manufacturing capability. It is key to have sensor technology that can perceive the position of a part under unconstrained motion and either inspect the part or direct a robot to manipulate it while still in motion. The technology must also be able to perceive possibly co-existent humans and other moving objects and generate corresponding safety actions. To enable this functionality, a method is needed to continuously measure the six-degree of freedom (6DOF) location and orientation of an unconstrained moving object. Existing affordable pose measurement systems are too inaccurate, brittle and slow. No standards exist to evaluate the accuracy of such systems or to guide users in their adoption. There are a number of candidate non-contact technologies with good potential including stereo cameras, laser triangulation, structured light, interferometry, scanning ladar, flash ladar and monocular geometric matching smart cameras, but there is a lack of common terminology and common vendor accuracy measurements leading to confusion in user comparisons and marketplace hurdles. As a result, few are in use and many manufacturing tasks are not automated or make use of humans and assistive devices. A reference standard for dynamic 6DOF pose measurement would advance the technology by establishing metrics for the evaluation of these systems and techniques. In addition to automated assembly tasks on a traditional line, advanced perception systems would be useful in material transport and other industrial tasks associated with manufacturing.

Terminology standardization is recognized as a means of facilitating next generation dynamic manufacturing. Given the size, rich history, and ongoing research and development efforts of manufacturing industry, there is abundant vocabulary that is either existent or evolving. For example, practitioners begin to use such terms as Next Generation Robots, but with different meanings. Some use the term to mean robots with the capabilities of higher payloads or inherent safety design. Standardization can be beneficial. In addition, there are terms that are developed in the defense and homeland security unmanned systems (UMS) community that can be explored for the manufacturing automation purposes as the industry is moving more and more toward autonomous operations and intelligent manufacturing for the sake of safety and productivity. The Terminology part of the NIST Autonomy Levels for Unmanned Systems (ALFUS) Framework [B.h.1] presents these opportunities that should be worthy of exploration.

The ability to measure the positions and orientations of components as they move would 
result in considerable cost savings in applications such as automobile manufacturing by replacing expensive fixed installations with more intelligent combinations of sensing and automation. The ability would also enable greater flexibility and adaptability for U.S. manufacturers and better enable them to compete with foreign firms where greater investments have been made recently in robotic technology. There would be substantial, immediate benefits in industry segments like automotive and airplane assembly, but the technology is fundamental and could be widely applied. A reference standard would also assist the academic community in establishing clear performance metrics for research systems and algorithms.

\section{WORKSHOP INFORMATION}

\subsection{Participating Organizations}

Sponsoring Organizations

National Institute of Standards and Technology

Loyola College in Maryland

SICK, Inc.

End Users - Presentation Topics

General Motors - Autonomous Assembly

Washington Post - Newspaper Manufacturer

US Postal Service - Distribution

General Dynamics Electric Boat - Shipbuilding

SICK, Inc. - Next Generation Robots

Ford Motor Company - Process Modeling

Academics and Government - Presentation Topics

Carnegie Mellon University - AGV Control

Purdue University - Dynamic Visual Servoing

SRI International - Visual Simultaneous Localization and Mapping (VSLAM)

NIST Building and Fire Research Laboratory - 3D Imaging Systems Standards

Sensor, Robot and AGV Manufacturers - Current and New Products

Sensors:

SICK, Inc. - Non-Contact Measurement

Automated Precision, Inc. - 6 Degrees of Freedom (DOF) Measurement

TYZX - Stereo Imaging

Shafi (USA) - Stereo Imaging

Mesa Imaging - Range Camera

Robot Arms:

Barrett - WAM Robot Arm
FANUC $\quad$ Robotics
Vecna - Bear Robot


Automated Guided Vehicles:

Egemin Automation - Automated Guided Vehicles

FMC - Automated Guided Vehicles

Demonstrations of Products

Mesa Imaging - Range Camera

API - 6D measurement system

SICK - laser measurement

TYZX - 3D Stereo Vision Platform

Barret - robot arm

\subsection{Workshop Agenda}

\section{Wednesday, October 10, 2007}

8:00-8:20 AM Welcoming Remarks (5 min. each) - Room 230/210

Opening Remarks: Roger Eastman, Professor

Loyola College in Maryland

NIST, Manufacturing Engineering Laboratory (MEL), Intelligent Systems Division

Overview: Al Wavering, Acting Deputy Director, MEL

Overview: Roger Bostelman, Manager

Intelligent Control of Mobility Systems Program

8:20 - 10:00 AM End Users (15 min. each +5 min. Q/A, set-up)

What are the main, prioritized manufacturing issues?

End User 1 - Automobile Manufacturer

Roland Menassa, General Motors - Autonomous Assembly

End User 2 - Newspaper Manufacturer

Conrad Rehill, Washington Post

End User 3 - Distribution

Joyce Guthrie, USPS

End User 4 - Shipbuilding

Ken Fast, GDEB (presented by Roger Bostelman, NIST)

End User/Facilitator 5 - Next Generation Robots

Brian

McMorris, SICK, Inc.

10:00 - 10:20 AM Q\&A / Discussion

10:20 - 10:30 AM Break 
10:30 - 11:10 AM Academia and Government (10 min. each)

Past / present research

Carnegie Mellon University, George Kantor - AGV Control

Purdue University, Avi Kak - Dynamic Visual Servoing

SRI International, Moti Agrawal - VSLAM

NIST Building and Fire Research Laboratory, Alan Lytle - Standards

11:10 - 11:30 AM Q\&A / Discussion

11:30 - 12:15 PM Sensors, Robots and AGV's - (3-5 min. each)

Current/New Products to Support Dynamic Measurement and Control

for Autonomous Manufacturing

Sensors:

SICK,

TYZX,

Shaf

\author{
Brian McMorris \\ Automated Precision, Inc., Tom McLean \\ Gaile G. Gordon \\ i (USA), Adil Shafi
}

Mesa Imaging - Range Camera, Peter Hunt

Robot Arms: Barrett, FANUC Robotics and Vecna, Claude Dinsmoor

Automated Guided Vehicles: Egemin Automation and FMC

12:15 - 1:45 PM Lunch and Exhibits - Room 230/210

Demos of products: - Rooms 208, 251

- Mesa Imaging - Range Camera

- API - 6D measurement system

- SICK - laser measurement

- TYZX - 3D Stereo Vision Platform

- Barret - robot arm

1:45 AM - 1:55 PM Charge to Breakouts Groups

Roger Eastman, Breakout information

2:00 - 3:45 PM Breakouts

Breakout Groups to Deliberate and Draft Research Recommendations and Performance Metrics Requirements

A. Requirements for dynamic 6DOF metrology for automated general assembly

Moderators: Roger Eastman, Loyola University and Tsai Hong, NIST

Room : 262

B. Control and perception needs for automated guided vehicles

Moderators: Roger Bostelman and Stephen Balakirsky, NIST

Room $\quad: 270$ 
C. Perception needs for real-time process monitoring and control

Moderators: Mike Shneier and Hui-Min Huang, NIST

Room: 280

D. Robot arms, their subcomponents and controls needed for dynamic

m anufacturing

Moderators: Fred Proctor and John Horst, NIST

Room: 272

3:45-4:00 PM Break

4:00 - 5:00 PM Plenary Session - Room 230/210

Breakout groups to present summaries

Thursday, October 11,2007

8:15 - 8:30 AM Welcoming Remarks

Roger Bostelman, NIST

8:30 - 9:00 AM End User 6 - Automobile Manufacturer

Dimitar Filev, Ford - Process modeling

9:00 - 10:30 AM End User Panel Discussion:

Where To Go From Here: Standards and Technology Roadmap

10:30 - 10:45 AM Break

10:45 - 11:45 AM Academic Panel Discussion:

Where To Go From Here: Research Roadmap

11:45 - 12:00 AM Wrap-up Summary

Tsai Hong, NIST

Where to go from here: Report and other follow on activities

12:00 PM Adjourn 


\title{
3. WORKSHOP RESULTS
}

\subsection{Day 1 Breakout Sessions Outcome}

The workshop organizers identified the following critical issues to be addressed by the participants:
A. dynamic metrology and perception on the assembly line,
B. needs for enhanced robot control systems to operation in dynamic environments,
C. the requirements for advanced AGV use in dynamic environments, and
D. real-time sensing to validate and update virtual simulations.

Four breakout groups were formed correspondingly. Each was assigned a central theme that highlighted a key issue in the envisioned dynamic manufacturing environment. Also provided were a corresponding vision and a preliminary information statement intended to foster discussions. Participants were assigned to the groups according to their affiliations. The objective is to have vendors, users, and developers evenly distributed in the breakout sessions to facilitate well-rounded viewpoints of the issues. Note that the actual participations were different from the assigned as some participants felt that they had contributions to offer for the other groups and attended multiple sessions.

The group activities and results were described in the following sections.

\section{A. Requirements for dynamic 6DOF metrology for automated general assembly}

Moderators: Roger Eastman, Loyola College in Maryland and Tsai Hong, NIST

Group Assignments:

Name
Jane Shi
Jonathan St. Clair
Peyush Jain
Steve Freedman
Peter Kamp
Kam Lau
Zaifeng Chen
Dave Strzegowski
Jamie Nichol
Roger Eastman
Avi Kak
Tsai Hong
Daniel Dementhon

\author{
Organization \\ General Motors (GM) \\ Boeing \\ Goddard Space Flight Center \\ SICK \\ SICK Germany \\ Automated Precision, Inc \\ Automated Precision, Inc \\ General Dynamics Robotic Systems (GDRS) \\ Vecna Technologies \\ Loyola Univ. \\ Purdue University \\ NIST \\ National Science Foundation
}




\section{Information Provided Prior to the Workshop}

$\underline{\text { Vision }}$

To achieve flexible and reconfigurable automation of manufacturing processes through sensor technology that can perceive the poses of a part in motion by dynamic 6DOF pose measurement.

Preliminary information to foster discussion:

In many manufacturing assembly environments, parts are delivered on moving lines and must be picked up and attached to the corresponding part being manufactured, which is also moving. Current technology typically requires the line to stop while an action is performed or a measurement taken. To achieve flexible and reconfigurable automation of assembly processes, it would help to have sensor technology that can dynamically perceive the pose of a part, in 6DOF. What issues may be involved in using improved or advanced sensor technologies to achieve accurate and robust 6DOF measurements? Are current sensor technologies up to the task? Where are technological advances needed? In new sensors? In the improvement of current sensors through factors such as advanced resolution and frame rate? In improved algorithms for motion and pose analysis? Will the solution require the fusion of data from multiple sensors?

\section{Results of 6 DOF Metrology Group Discussions}

During their discussion, the workshop participants listed the following elements as important to the development and success of the envisioned new technology.

$\underline{\text { Roadblocks and Challenges }}$

These elements were determined to be problems faced by the new technology:

- Advanced 6DOF perception is a young technology, in its initial phases and with maturity perhaps 10 years away, and will face a question of economic viability.

- An entire commercial ecosystem will be required, involving sensor manufacturers, system integrations, robots manufacturers, standards organizations, and others.

- A successful system will need to exhibit continuous adaptation to changing conditions, as such, the system will have to be very complex, hence very difficult and expensive to develop.

- A successful system will need to be robust and have clearly specified capabilities and limitations. The extremely large numbers of possible parts that may be needed and situations that may occur in the envisioned dynamic and unstructured manufacturing environments will make this new technology difficult to achieve.

\section{Concrete Scenarios}

The participants put together a few cases of interest to manufacturers for the phased development and evaluation of 6DOF sensors. The scenarios are listed in order of difficulty:

1. First case: mating of two rigid parts under dynamic conditions, as typically encountered in automotive general assembly.

2. Second case: mating of a flexible part, such as a hose, to a moving rigid part. 
3. Third case: mating of parts, rigid or not, that would involve complex path planning, such as an attachment inside a vehicle or base assembly.

4. Fourth case: manipulation of non-rigid attachments associated with parts, such as an electronic automotive part with a number of wiring leads attached.

\section{$\underline{\text { Solutions }}$}

The group listed the following characteristics as important to a successful advanced perception solution:

- A successful 6DOF perception system should be comprehensive, pervasive, redundant, multi-level and multi-resolution. Such a system can perceive an entire scene, sense the position of a part over a wide range of distances, and be robust against the isolated failure of components or sensors.

- A successful sensor system that is faster and more accurate might be a substitute for more intelligence, as better information about the world can reduce the requirements for reasoning.

- A successful perception system should be a part of an overall solution, balanced with other concerns. For example, the need for more accurate sensing during a robotic pickup operation may be mitigated by improving the compliance of the grippers, allowing less precise sensing.

What types of sensors?

The group produced an initial listing of the categories of sensors that are likely to be used in a solution, shown below:

- Single camera

- Multiple cameras (stereo)

- Range sensors

- Structured light

- Laser scanning

- Flash ladar

- Force/torque sensors

$\underline{\text { Research needs }}$

The group considered areas in which new research will be required to solve the problem. The following are its findings:

- Research should be conducted in the use of multiple, heterogeneous sensors, including fusing sensor data, enabling cooperation among sensors, and arbitrating between sensors with conflicting data.

- Research should be conducted in the modeling of sensor performance in static and dynamic environments. Research should also be conducted in the subsequent estimation methods of the sensory measurement confidence levels.

$\underline{\text { Sensor Requirements/Metrics }}$

While the number of possible manufacturing applications is very large and hard to easily characterize, the group made some initial progress in the area of spatial and temporal tolerances, as described below:

- For general assembly, the position of an object should be measured to about $0.32 \mathrm{~cm}$ 
$(1 / 8$ in).

- For specialized assembly tasks, these tolerances may be tightened to $1 / 4000 \mathrm{~cm}$ (1/1000 in).

- The latency of the sensor system should be minimal, ideally near 0 . All things equal, a higher sampling rate is better.

- The performance of a sensor system should be commensurate with the motion statistics of the manufacturing environment, taking into account range, velocity and accelerations.

Standards Needs

- The pose of a part needs to be measured with a specified standard deviation with respect to each of the 6 degrees of freedom when the line is moving at a given speed with specified statistical properties.

\section{B. Control and perception needs for automated guided vehicles (AGVs)}

Moderators: Roger Bostelman and Stephen Balakirsky, NIST

Group Assignments:

Name

James Wells

Joyce Guthrie

Brian McMorris

Peter Hunt

Joe Stanford

Gaile G. Gordon

William T. Townsend

Mark Longacre

Brad Byle

Andreas Hofmann

Moti Agrawal

George Kantor

Roger Bostelman

Steve Balakirsky

\author{
Organization \\ GM \\ USPS \\ SICK \\ Mesa Imaging \\ Automated Precision, Inc \\ TYZX \\ Barrett Technology \\ FMC \\ Egemin Automation \\ Vecna Technologies \\ SRI International \\ CMU \\ NIST \\ NIST
}

\section{Information Provided Prior to the Workshop}

Vision

To achieve improved AGVs that can be more rapidly deployed and can operate in dynamic, unstructured environments

Preliminary information to foster discussion:

In their current form, AGVs are useful but limited on the tasks that they can perform. They are not able to access all unstructured areas of a plant, may require particular plant floor design to accommodate them, and may need special fixtures for loading and unloading. The problems become more difficult when people, parts and a mixture of AGVs are moving in the environment. What issues must be solved before the technology 
is universal, flexible and robust enough to support dynamic manufacturing? What are the issues in drive systems, in absolute and dynamic positioning systems, and in AGVcoordination among themselves, with central systems, and with humans? What are the issues in modeling and simulation, such as simulating the simultaneous motions of a fleet of AGVs to achieve high factory efficiencies? Are new, radical designs needed to perform required tasks, such as motion in spaces design primarily for humans or in carrying manipulators for tasks like installing a wiper blade on a moving automotive assembly line?

\section{Results of 6 DOF Metrology Group Discussions}

During their discussion, the workshop participants listed the following elements as important to the development and success of the technology:

$\underline{\text { Roadblocks }}$

The following elements were determined to be problems and questions faced by the new technology:

- A useful AGV must be capable of localization, finding its location relative to a map well. Questions related to localization were determined to be:

o What is the best use of an internal GPS for absolute positioning and mapping?

o What is the process to find a vehicle location with respect to a known point (localization)?

o What tolerance is needed for the dynamic measurements? Would an accuracy or repeatability of $10 \mathrm{~mm}$ be adequate?

o Will localization be achieved with multiple sensors or one "magic" sensor?

0 Is there a need for retrofitting current facilities with localization capabilities to enable them to handle unstructured environments? For example, is there a need to convert from a rigid set of markers/tracks, installed on the floor or at other places, to a flexible AGV system with dynamic localization capabilities? If so, what would be the cost?

- Advanced AGVs will need to have functionality that meets user demand and costs that are affordable. Questions are:

0 What's keeping users from using more AGV's, AGC's (smart-carts), and forklifts/tuggers? Is there a chicken-and-egg problem, where apparent lack of demand is holding back development?

0 What are the infrastructural costs to supporting an intelligent AGV?

o Is there a demand for robot arms on vehicles?

o What would be the advanced safety standards for mobile robots? There is a lack of clear definitions for safe operation of an AGV.

o Can AGVs be made taller to have a higher work volume, yet are still stable?

o Can they be made faster, yet still safe and have adequate stopping distances?

o Can they be made easy to use and flexible, so they can be quickly brought across assembly lines?

o Can they be made scalable, from low to high volume, and manual to autonomous in operation?

- Other technical issues in AGV development and acceptance:

o From the perspectives of real time control and scheduling, should the control be 
integrated on the vehicle or distributed in the workplace?

o Are there low cost sensors that are robust enough, come with adequate support, yet meet the new needs in safety and performance?

o Is battery technology holding back AGV performance?

0 Are there adequate standards for AGVs and military vehicles, along the lines of TRL (Technology Readiness Level) or ALFUS, for commercial use?

$\underline{\text { Required solutions and research needs }}$

- We need to develop better standards, performance metrics, and system specification methodology related to:

- Safety standards for AGVs to categorize and quantify risks to humans.

o Collaborative AGVs that work together on tasks.

o Capabilities of arms on vehicles that enable mobile manipulation.

- Specifications of plans, standard task vocabularies and levels of autonomy (ALFUS) to enable easier descriptions of vehicle capabilities.

o Criteria for ease and intuitiveness of AGV programming, including

- Better high-level programming languages,

- Improved user interfaces for direct teaching modes.

o Performance under varying environmental conditions, such as lighting.

o Localization and mapping, including

- How accurate must a vehicle be to safely navigate and tow objects, parts trays, and carts?

- How accurate is a map produced by sensors?

- How accurately can an AGV localize itself?

- We need lower cost and better vehicular technology for:

o Brake locks that can fully support both emergency and protective stops.

o Suspension and intelligent compensation technology that can accommodate variations in suspension. For example, soft suspension can lead to localization problems related to dead reckoning.

o Mobile manipulation with integrated $\operatorname{arm}(\mathrm{s})$ on vehicle.

o Coordinated control and planning for single or multiple AGVs.

o Sensors for functionality and safety.

\section{Information needs for real-time process monitoring and control}

Moderators: Mike Shneier and Hui-Min Huang, NIST

Group Assignments:

Name

Conrad Rehill

Jacqueline LeMoigne-Stewart

Stephan Schmitz

Clarence Burns

Jay Li

Adil Shafi

Eric Beaudoin
Organization

Washington Post

Goddard Space Flight Center

SICK Germany

Automated Precision, Inc

Automated Precision, Inc

Shafi (USA)

GDRS 
James Albus

Jeremy Zoss

Rama Chellappa

German Londono

Mike Shneier

Hui-Min Huang
NIST Fellow

Southwest Research Institute

University of Maryland

Purdue University

NIST

NIST

\section{Information Provided Prior to the Workshop}

Vision

To achieve manufacturing line efficiency and quality improvement by better acquisition and use of on-line (perception-based) and a priori (model-based) information.

Preliminary information to foster discussion:

Knowledge of the current state of the assembly line can be critical in achieving efficiency, in avoiding bottlenecks and quality problems, in planning for higher efficiencies or line redesign, and in keeping virtual line simulation coordinated with the real-world. Advanced sensors may be able to play a role in monitoring the assembly line and performing dynamic metrology on parts in motion, advancing the current field of machine vision to better adapt to unstructured environments. What information do users need from perception systems to manage and control their process? What does the virtual assembly information contribute to the process? What are the dynamic metrology capabilities in perception systems that improve process control in unstructured environments?

\section{Results of 6 DOF Metrology Group Discussions}

$\underline{\text { Roadblocks }}$

- The vision statement was found to be in line with the industrial problems and no adjustments were made.

Problems:

- The labor force has a shortage of workers skilled in automated machine operation.

- It is difficult to integrate new technology (machine vision, etc.) into existing systems.

- Intelligent equipment is expensive and hard to evaluate.

- Knowledge acquisition and representation are difficult problems for automated manufacturing.

- It is difficult for automated systems to detect and identify critical events from current information sources.

- Current safety requirements can hinder human operations in robotic environments there is a need to clearly identify what happens and take actions to mitigate the effect. For example, having humans nearby should not necessarily shut the robots down.

- Small batch jobs and customized orders exemplify the problem of the variety of things to be measured. These point to the need for automation.

- Equipment can malfunction in dirty environments. Examples include simple situations like dust on sensors and uneven floors that interfere with AGV dead reckoning.

- There are many sources of errors and exceptions. For example, error models can 
become ineffective.

- It is important, but difficult to, have post-operation verification - make sure that the operation succeeds what is supposed to be done, especially in safety related issues.

- With the contributions of a representative of the newspaper industry, the discussion yielded a number of fruitful items specific to that industry. They are:

o Inserts of newspapers count as a significant portion of a newspaper company's revenue. An important requirement is to deliver them to where the advertisers want, according to zip codes, streets, or other demographic concerns.

o Heavy time constraints exist to identify particular pallets for inserts, followed by their loading and delivery.

o There is a need to label particular bundles for accurate tracking. Currently need to produce 700,000 copies daily.

o Vision systems may be a good technology to identify and retain knowledge about the bundles.

0 Bundles may break in the process and mess up the counting system.

o Current accuracy rate is about $98.6 \%$ for the Washington Post AGV operation.

$\underline{\text { Solutions }}$

- Need to understand and model the full operation of AGVs. All the possible exceptions must be listed and programmed into the systems to become parts of the model.

- Need metrics to evaluate the robustness/costs/performance of the implemented algorithms.

Research needs

(Did not get to this topic.)

Requirements/Metrics

(Did not get to this topic.)

$\underline{\text { Standards }}$

(Did not get to this topic.)

\section{Automated manipulators, their subcomponents and controls needed for dynamic manufacturing}

Moderators: Fred Proctor and John Horst, NIST

Group Assignments:

Name

Maravas, Michael

Theodore Bugtong

Wolfgang Bay

Yuanqun Liu

Tom McLean

Mark Bankard

\author{
Organization \\ USPS \\ Goddard Space Flight Center \\ SICK Germany \\ Automated Precision, Inc \\ Automated Precision, Inc \\ GDRS
}


Brian Zenowich

Claude Dinsmoor

Daniel Theobald

Johnny Park

John Horst

Fred Proctor
Barrett Technology

GE Fanuc

Vecna Technologies

Purdue

NIST

NIST

\section{Information Provided Prior to the Workshop}

$\underline{\text { Vision }}$

- To achieve better flexibility and ease of deployment and operation of automated manipulators in dynamic environments

- Customers: domain experts, not technology experts, need assistance in technology choices.

- Deployment benefits accrue mostly for one-of-a-kind installations; operation benefits accrue for everyone.

- Dynamic: everything can change: environment, process, product

Preliminary information to foster discussion:

To achieve better flexibility and ease of deployment, robot arms, intelligent assist devices and other programmable devices for automated handling of material will need to operate more and more in dynamic environments where people, parts, conveyers and AGVs are moving. In the advanced case, robot arms will be mounted on AGVs or humanoid platforms and operating in a dynamic environment where they will be interacting with people. What issues are there in advancing the technology so this can be accomplished safely and efficiently? Are there issues in arm design, in programmability, or in standardization of interface between robot controller and other system elements such as PLCs and sensors? Are there issues in underlying control theory or calibration and validation of system performance? What elements of the systems will need to be enhanced to take advantage of advanced 3D sensors?

\section{Results of 6 DOF Metrology Group Discussions}

Roadblocks

The group voted to prioritize the identified roadblocks. Participants could vote for multiple items. The size of a vote (following each item) represents a measure of group consensus towards prioritization. The ones without a vote were seen as either lower priority or not common issues across the entire industry.

- Achieving safe and flexible robotics and enabling collaboration with humans-resulting in new applications: 14

- Improving software-based safety chain, including possibly revisions to the existent standards. This step would enable the achievement of the other roadblocks on this list: 7

- Making AGVs with robot arms easy to use and to set up, including deployment and development: 8

- Enabling conformance tests, performance tests, and verification against a specification: 8 
- Eliminating incompatibility, e.g., issues with data exchange, connectors, programming languages: 5

- Dealing with obsolescence of AGVs.

- Hardening systems for work in dirty environments.

The highest-ranking requirements are further elaborated as below:

$\underline{\text { R1: Achieving safe and flexible robotics: enabling collaboration }}$

o Technologies needed: better collision sensing and avoidance.

o Metrics needed: what is damaging to a person? For example, the head injury criteria from auto industry might be applicable.

o Standards needed: software-based safety chain.

o Research needed: what constitutes safe behaviors (varies widely across applications and robot types); what are effective ways for robots and humans to collaborate?

R2: Ease of use, setup, including deployment and development

o Technologies needed: application development techniques that domain experts are familiar with.

o Standards needed: interface to robotic functions that support operations in a dynamic environment.

R3: Conformance tests, performance tests, verification against a specification

o Metrics needed: performance for the identified range of human-robot interactions; trust or perception of safety.

o Standards needed (other than the safety standard): motion detection, frame rates, reaction bandwidth, or any that supplements the current Robotic Industries Association (RIA) standards.

0 Research needed: trust and perception of safety, risk assessment. 


\subsection{Day 2 Wrap-up Discussion and Results}

Participants engaged in a discussion that summarized the first day's findings and looked to follow-up actions. The following are the results:

A. Needs identified during the workshop:

a. Better and more complete standards in the areas of:

- Interfaces for equipment and software interconnection

- Terminology - lists of common tasks and commonly used terms

- Evaluation metrics and methods for sensor, AGV, robot and overall system performance

The development efforts should be synchronized with the defense industry activities.

b. More use of scenarios and competitions to drive development:

- Industry associations could pool resources to establish challenges.

- Establish a list of the challenges and competitions for researchers to study.

- Simulation challenges are much easier to be set up. NIST runs one on a simulated AGV.

c. Manufacturers need to provide more information on automation issues and impacts:

- Form consortia to collect general issues and observations on challenges faced.

- Establish individual research on specific industry needs.

d. Better supporting technologies:

- Establish the capability to seamlessly run tests from virtual to real.

- Develop techniques to monitor the automation progress based on requirements and metrics.

B. Action Items and Associated Volunteers

a. Overarching goal: develop standards for interfaces, performance, metrics, and terminology, e.g., robot safety and software components.

b. Become involved in RIA standard processes:

- Jim Wells, Roger Bostelman, and Brian McMorris will begin looking into how to start process of developing future standards. The types of standards lab tests that can be beneficial to the industry include: feasibility, conformance, and performance.

- Hui-Min Huang will look into terminology standards: identify manufacturing terms, look for existing standards.

c. Create well-defined challenge scenarios:

- Joyce Guthrie, Jane Shi, Roger Bostelman, and Stephen Balakirsky.

- Non-rigid components, e.g., carpets, cable harnesses, hoses are to be included.

d. Develop cooperation with industry associations and systems integrators to target research on "needs:" 
- Automotive Industry Action Group (AIAG), Mechanical Contractors Association of America (MCAA), Material Handling Industry of America (MHIA), RIA, and Society of Manufacturing Engineers (SME) are among the relevant associations.

- Jim Wells has experience in this and should be a lead.

- Names of systems integrators can be forwarded to Jim Wells and Roger Bostelman.

e. There seems to be no trade organizations that focus on systems integrators.

f. Set up Workshop mail group:

- Fred Proctor

g. Complete workshop report:

- Workshop organizers

h. Next workshops/meetings and other interesting forums -

- Combine need-focused meetings with major conferences.

- Robot Industry Forum, Orlando, Nov. 2007

- IEEE Conference on Computer Vision and Pattern Recognition (CVPR, which tends to be academic), International Manufacturing Technology Show (IMTS, September 2010), International Robots, Vision \& Motion Control Show hosted by RIA (June 2009) [2, 3, 4]. 


\section{INITIAL ACTION ITEMS RESULTS}

As of this report date, work has already begun on the identified action items and the results are described in the following sections.

\subsection{Formulating New Projects for Identified Critical Needs}

To address a key sensory requirement for dynamic manufacturing, NIST has embarked on a dynamic 6DOF pose measurement project. The goal is to devise a method to continuously measure the locations and orientations of an unconstrained moving object. Current automated assembly systems typically measure the pose of an object only in highly constrained situations, such as parts moving at a fixed speed in a rigid conveyance, or by stopping the assembly line to sense the precise position of the part. Locating and tracking an arbitrary object under unconstrained motion is very difficult as majors issues exist for most of the sensing technologies. For example, optical camera-related technology may involve loss of 3D information through projection. The 6DOF related technology must require the pose to be reconstructed from the data in real-time for which the equations and algorithms are not yet fully understood. A reference standard for dynamic 6DOF pose measurement would advance the technology by establishing metrics for the evaluation of these systems and techniques.

The project will develop methods for continuous measurement for manufacturing applications such as automobile manufacturing and for evaluating the sources of error in the measurements, including finding out how to minimize the errors. This will require techniques to calibrate the reference and test systems, to synchronize measurements for comparisons, to evaluate the raw sensor data that is used to compute 6DOF pose, and to track the contribution and propagation of errors in subsystems.

The ability to measure the positions and orientations of components as they move would result in considerable cost savings in applications such as automobile manufacturing. The ability will allow expensive fixed installations to be replaced with more intelligent combinations of sensing and automation, and, thus, better enable US manufacturers to compete with foreign firms where greater investments have been made recently in robotic technology. Although our current focus is on the substantial, immediate benefits in industry segments like automotive and airplane assembly, the technology is fundamental and could be widely applied. A reference standard would also assist the academic community in establishing clear performance metrics for research systems and algorithms.

Roger Bostelman and Stephen Balakirsky began coordinating space allocation with the NIST machine shop and designing a testbed for a NIST exploratory project on vehicle navigation through unstructured environments. The testbed will include a robot arm mounted on overhead rail to enable a number of automation scenarios. This testbed will serve as a beginning for well-defined scenario testing as they arise.

\subsection{Further Investigation on Industrial Needs}

This workshop and its proposed future standards development effort were discussed in the $15^{\text {th }}$ Annual Robotics Industry Forum that Roger Bostelman, Brian McMorris, and Jim Wells 
attended in Orlando on November 7-9, 2007.

Jim Wells discussed with RIA participants at the Orlando Robotics Industry Forum about research on "needs."

\subsection{Identification of Current Standards}

Hui-Min Huang took an action item of researching the current robotic standards in the areas of vocabulary for tasks and systems. The results include a collection of ISO, ANSI, and RIA standards that mostly deal with low level devices, coordinate systems, geometry/kinematics, programming, and limited performance evaluation. They are summarized later in this section. This finding points to a possible broad-scope structure for robotic standards that may encompass multiple levels of abstraction for the knowledge. Aspects of task structures, a general purpose unmanned systems terminology, and ontology may all be covered in the structure. It would be interesting to find out how the concept of autonomy levels can be applied in the manufacturing domain. Corresponding terms like Unmanned Flexible Manufacturing System (UFMS), and Unmanned Workstation (UWS) might be explored to embed various levels of operator interactions.

\subsubsection{Terminology and system characteristics}

The following standards are identified and are listed according to the publishing organizations.

\section{ISO}

ISO 14539: 2000

Manipulating industrial robots -- Object handling with grasp-type grippers -- Vocabulary and presentation of characteristics

Categories: types of handling, grasps, coordinate systems and sensing in object handling, types of grasp-type grippers, types of end effectors, elements of grasp-type grippers, types of grasp-type grippers, types of fingers, finger control, clamping elements, robot interfaces, safety in grasps and grasping

ISO 9787: 1999

Manipulating industrial robots -- Coordinate systems and motion nomenclatures Content: world, base, mechanical interface, and tool coordinate systems, robot motion, robot axes

\section{ISO 9946: 1999}

Manipulating industrial robots -- Presentation of characteristics

Manufacturer shall provide: application, power source, mechanical structure, working space, coordinate system, external dimension and mass, base mounting surface, mechanical interface (how end effectors are mounted on robotic wrists), control, task programming and program loading, environment, velocity, resolution, performance criteria, safety

ISO 11593: 1996

Manipulating industrial robots -- Automatic end effector exchange systems -- Vocabulary 
and presentation of characteristics

Categories: external shape, main dimension, position and orientation in coupling

procedure, coupling and releasing forces, load, magazine interface, tool exchange time

ISO 8373: 1994/1996

Manipulating industrial robots - Vocabulary

An amendment ISO 8373:1994/Amd 1:1996 and a corrigendum ISO 8373/Cor.1:1996

followed.

Categories: general terms, mechanical structure, geometry and kinematics, programming and control, performance

\section{AIAA:}

R-103: 2004

AIAA Recommended Practice: Terminology for Unmanned Aerial Vehicles and Remotely Operated Aircraft

\section{AIAA S-066}

Standard Vocabulary for Space Automation and Robotics (1995)

\section{ASTM:}

$\underline{\text { E } 2521-07 \mathrm{a}}$

Standard Terminology for Urban Search and Rescue Robotic Operation

$\underline{\text { E } 2544}$

Standard Terminology for Three-Dimensional (3D) Imaging Systems

\section{IEEE:}

IEEE 100-2000

The Authoritative Dictionary of IEEE Standards Terms (Seventh Edition, 2000)

\section{NIST:}

NIST SP 1011-I-2.0

Autonomy Levels for Unmanned Systems Framework, Volume I: Terminology, Version 2.0

\subsubsection{Safety and performance}

\section{ISO:}

ISO 10218-1, -2: 2006

Robots for industrial environments - Safety requirements

ISO 9409-1, -2, -3: 2004

Manipulating industrial robots - Mechanical interfaces

$\underline{\text { ISO 9506-1, -2: } 2003}$

Industrial automation systems - Manufacturing Message Specification 
ISO 9283: 1998

Manipulating industrial robots - Performance criteria and related test methods

\section{ANSI/RIA}

ANSI/RIA R15.05-1: 1990

Point-to-Point and Static Performance Characteristics - Evaluation

ANSI/RIA R15.05-2: 1992

Path-Related and Dynamic Performance Characteristics - Evaluation

ANSI/RIA R15.05-3: 1992

Reliability Acceptance Testing - Guidelines

\section{NIST:}

NIST SP 1011-I-2.0

Autonomy Levels for Unmanned Systems Framework, Volume II: Framework Models, Version 1.0

\subsubsection{D metrology}

Existing standards for 3D metrology, useful in defining terminology, artifacts and protocols that might be relevant to this effort exist [6]. In addition, there are ongoing efforts for developing performance standards for imaging systems. Below list three of these efforts:

- ASTM: 3D imaging sensors [7]

The ASTM Committee E57 on 3D Imaging Systems has been investigating standards for 3D imaging sensors, with the Building and Fire Research Lab (BRFL) at NIST taking a leadership role. The BRFL conducted workshops with sensor vendors and other interested parties in 2003, 2005 and 2006, and has done work to define terminology and initial protocols. The current focus is on static, large-scale metrology.

- IACMM: Non-contact metrology

The International Association of Coordinate Measurement Machine Manufacturers (IACMM) is supporting work on Optical Sensor Interface Standard (OSIS). This standard is intended to aid the integration of non-contact sensor technologies into traditional contact coordinate measurement machines. The standard has three elements on physical interfaces, software interfaces, and calibration. The latter effort covers accuracy specification and validation for the 3D data from non-contact sensors. The scope of this effort may cover 3D imaging systems of interest and dynamic and 6DOF performance is not emphasized. NIST participates in this project.

- EMVA: Machine vision sensor performance The European Machine Vision Association (EMVA) has the 1288 standard effort, "Standard for Measurement and Presentation of Specifications for Machine Vision Sensors and Cameras." The scope of the standard currently covers monochrome digital area scan cameras and should be extended to line and color cameras. The format could be a model for 
reporting 3D imaging performance. The standard is developed in a modular fashion, with each module defining a physical sensor model for characterizing sensor response, a protocol for testing the characteristics, and a format for presenting and analyzing the results. 


\section{REFERENCES}

1. Autonomy Levels for Unmanned Systems (ALFUS) Framework, Volume II: Framework Models Version 1.0, NIST Special Publication 1011-II-1.0, Huang, H., Ed., National Institute of Standards and Technology, Gaithersburg, MD, December 2007

2. http://www.cvpr.org/

3. http://www.imts.com/

4. http://www.robotics.org/

5. Hong, T.S., et al., Dynamic metrology: Evaluation of 3D Imaging Systems for Dynamic Sensing Applications in Manufacturing, NIST Draft Report, January 2009

6. Proceedings of the LADAR Calibration Facility Workshop June 12 - 13, 2003, NIST Internal Report, NISTIR 7054, Cheok, G., Ed., October 2003

7. http://www.astm.org/COMMIT/COMMITTEE/E57.htm 


\section{APPENDIX：WORKSHOP PRESENTATIONS}

Overview: Roger Bostelman, Manager

Intelligent Control of Mobility Systems Program ....

End Users

Automobile Manufacturer

Jane Shi, et al., General Motors - Autonomous Assembly .................... 34

Newspaper Manufacturer

Conrad Rehill, Washington Post .................................... 41

Distribution

Joyce Guthrie, USPS ................................................. 48

Shipbuilding

Ken Fast, GDEB - presented by Roger Bostelman, NIST ............................... 51

Next Generation Robots

Brian McMorris, SICK, Inc.

Academics and Government

Carnegie Mellon University, George Kantor - AGV Control .................... 68

Purdue University, Avi Kak - Dynamic Visual Servoing ................................ 75

SRI International, Moti Agrawal - VSLAM ................................... 88

NIST Building and Fire Research Laboratory, Alan Lytle - Standards ................... 97

Sensors, Robots and AGV's

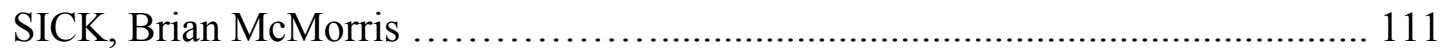

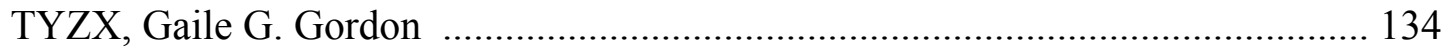

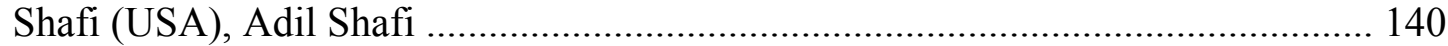

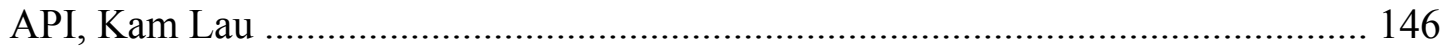




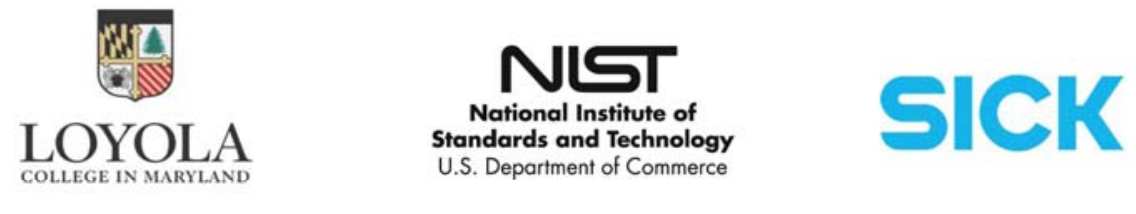

\title{
Dynamic Measurement and Control for Autonomous Manufacturing Workshop
}

\author{
Roger Bostelman, Tsai Hong, Stephen Balakirsky, \\ Elena Messina, Hui-Min Huang, NIST \\ Roger Eastman, Loyola College in Maryland \\ Brian McMorris \& Steve Freedman, SICK
}

Loyola College in Maryland, Columbia, MD

October 10-11, 2007

workshop slide \#1

\section{Vision}

- "Industrial robots guided by machine vision have the potential to revolutionize manufacturing processes, improving repeatability, cycle rate, reliability and safety on the plant floor, while reducing costs associated with labour and fixturing."

- Vision-guided robotics - March/April 2007 - Examining the technology's impact on the plant floor

- By Mary Del Ciancio

- "with vision guidance, robots can be deployed increasingly in places where robotic automation was not imaginable before, and you can see that this has a powerful effect on the landscape of manufacturing and the way we will lay out our plants of the future."

- Babak Habibi, President, Braintech 


\section{Roadblocks}

- lighting - ambient, sunlight, low light, part appearance

- training

- integration

- physical constraints of the sensor system

- location/logistics - where the system will go and who will operate it.

- financial issues - system cost, ROI

- practical issues - upkeep, maintenance and training

- control of the environment - airborne particles, abrasives, vibrations

- timing - e.g., latency control between the robot controller

- data type -2D, 3D

- communications - with robots, single or multiple sensors

- fixtureless/moving parts

- ....

workshop slide \#3

\section{Industrial Robot Roadblocks}

- $95 \%+$ of industrial robots are used without sensors in the outer loop

\section{Robot Challenges}

- Systems Integrations - largest portion of robot supply chain

- Largely disconnected from robot providers

- There are few established standards for system design

- Few tools available for comprehensive modeling

- Need programming and I/O support

Henrik I Christensen KUKA Chair of Robotics, Georgia Tech hic@cc.gatech.edu 


\section{Workshop Challenge}

- How do we address these roadblocks?

- Research

- Technology

- Standards

- Performance Metrics

\section{Current}

- automotive industry - assembly and processing of engine and body components.

- food industry - pick products from conveyors for packaging into individual containers or cartons.

- pharmaceutical industry - locate medical supplies on moving belts for packing into shipping cartons.

- metalworking industries - finding metal castings on pallets and loading $\mathrm{CNC}$ machines to make finished component products

- bin-picking applications today that just a few years ago were thought to be impossible." Roney:

- "Material handling is the low-hanging fruit that this technology can be used to capitalize on today,

- Typical [2D] applications [are] picking from a stationary or moving conveyor, pallet loading/unloading, conveyor tracking, and component assembly

- Typical [3D] applications are auto-racking and bin-picking. We are also seeing interest for robotic deburring and material removal applications (e.g., find and deburr parts) McLauglin, Boatner 


\section{WELCOME! \\ Attending Organizations}

- National Institute of Standards and Technology

- Loyola College of Maryland

- SICK - US and Germany

- General Motors

- Ford

- Washington Post

- US Postal Service

- Goddard Space Flight Center

- Mesa Imaging

- Automatic Precision, Inc.

- TYZX

- Shafi (USA)
- General Dynamics Robotic Systems

- Barrett Technology

- GE Fanuc

- FMC

- Egemin Automation

- Vecna Technologies

- Southwest Research Institute

- Purdue University

- Stanford Research Institute

- Univ. of MD

- CMU

- National Science Foundation

\section{Final Agenda}

Wednesday, October 10, 2007

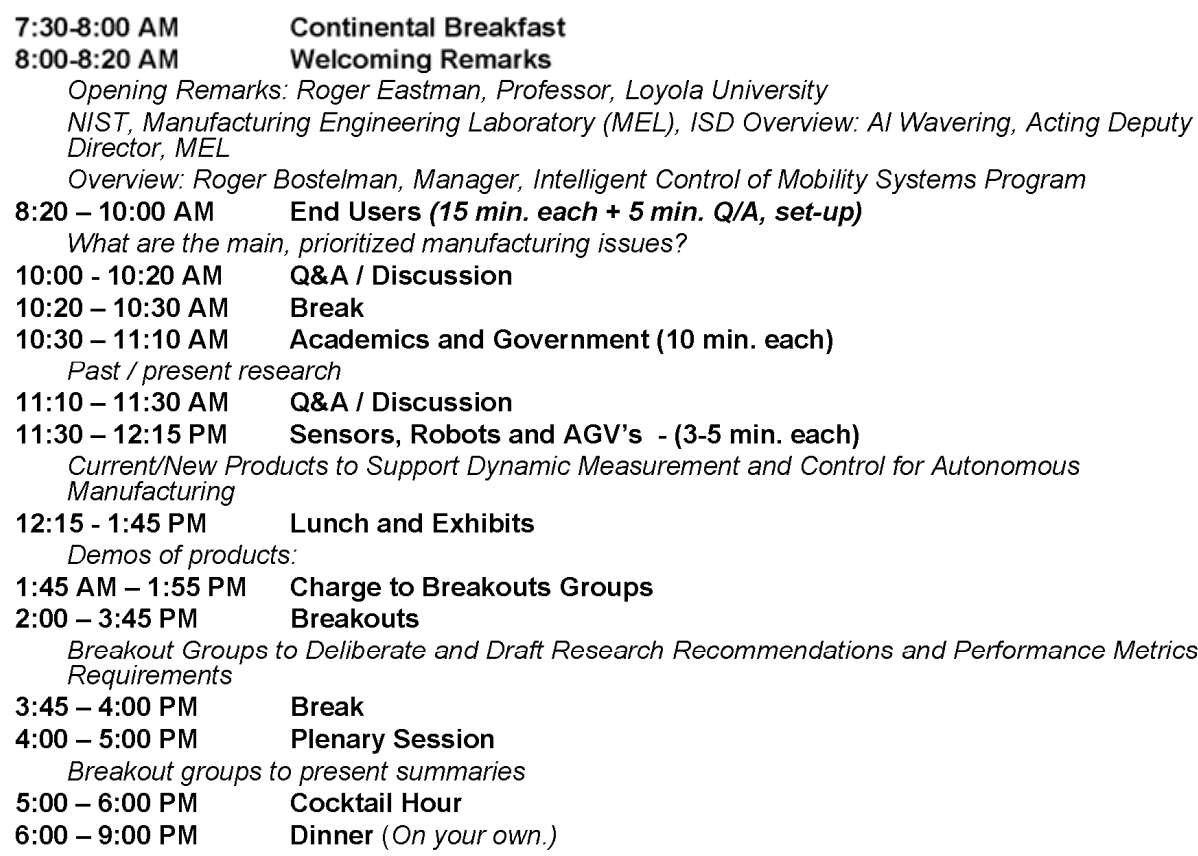




\section{Final Agenda \\ Thursday, October 11, 2007}

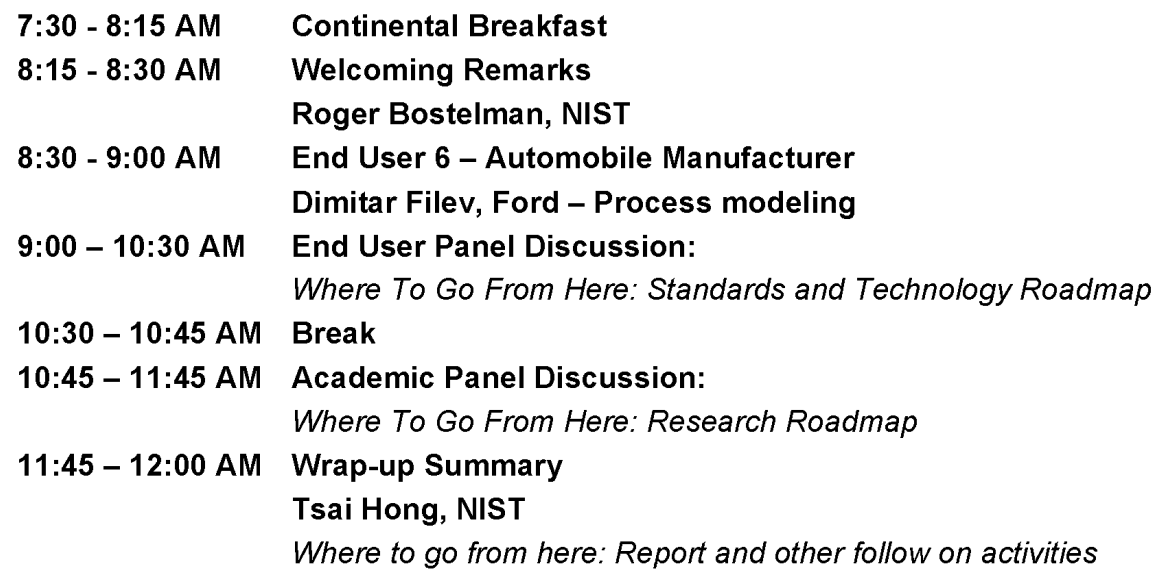

12:00 PM Adjourn

\section{Where To Go From Here?}

- Combine and Prioritize across breakouts:

- Research

- Technology

- Standards

- Performance Metrics 


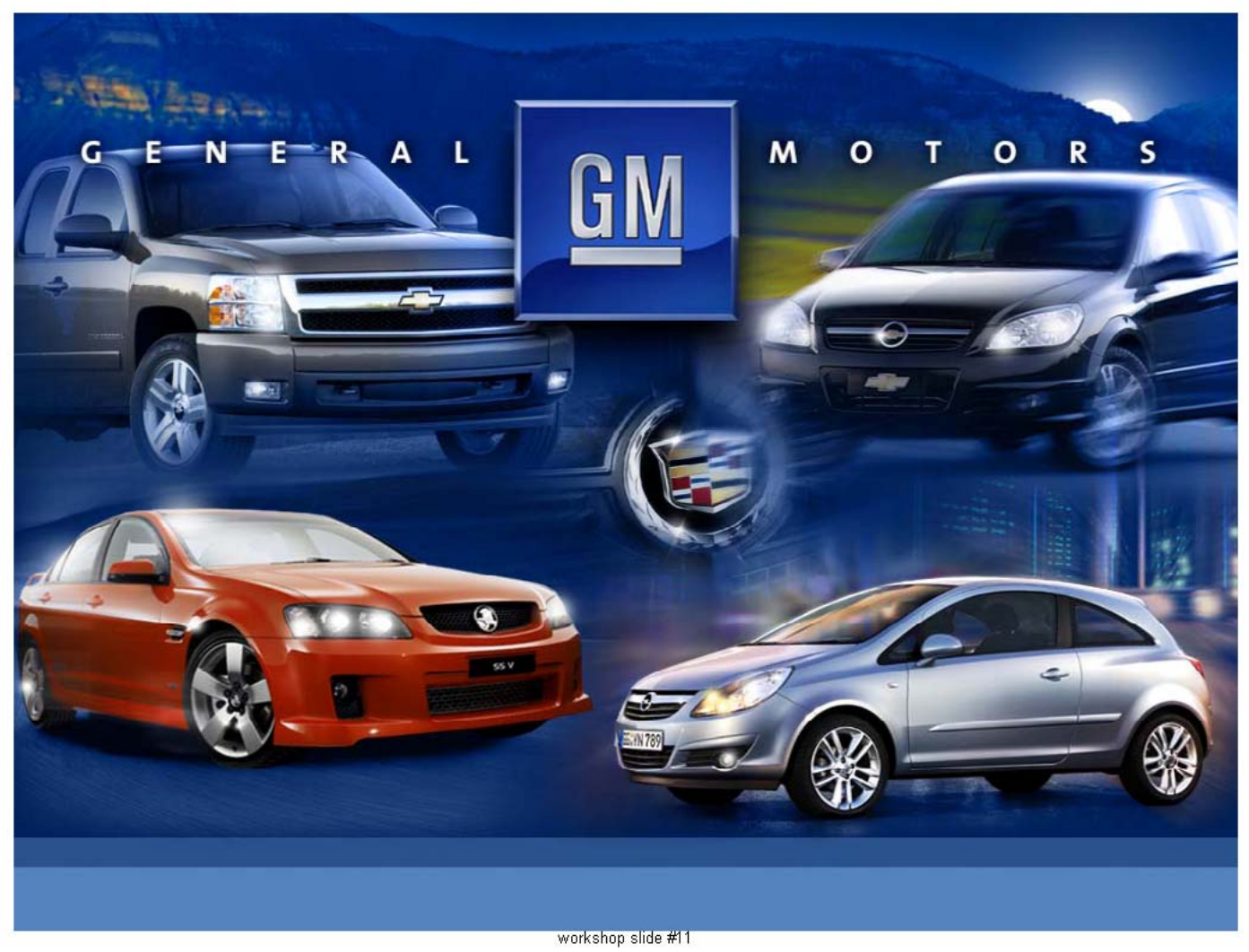

NIST Workshop on: Dynamic Measurement and Control for Automated Manufacturing October 10 $10^{\text {th }}, 2007$

Perception and Autonomous Robotic Assembly

Roland Menassa, Jane Shi, Jim Wells

Robotics, Tooling \& Equipment Mfg Systems Research Lab General Motors R\&D Center 


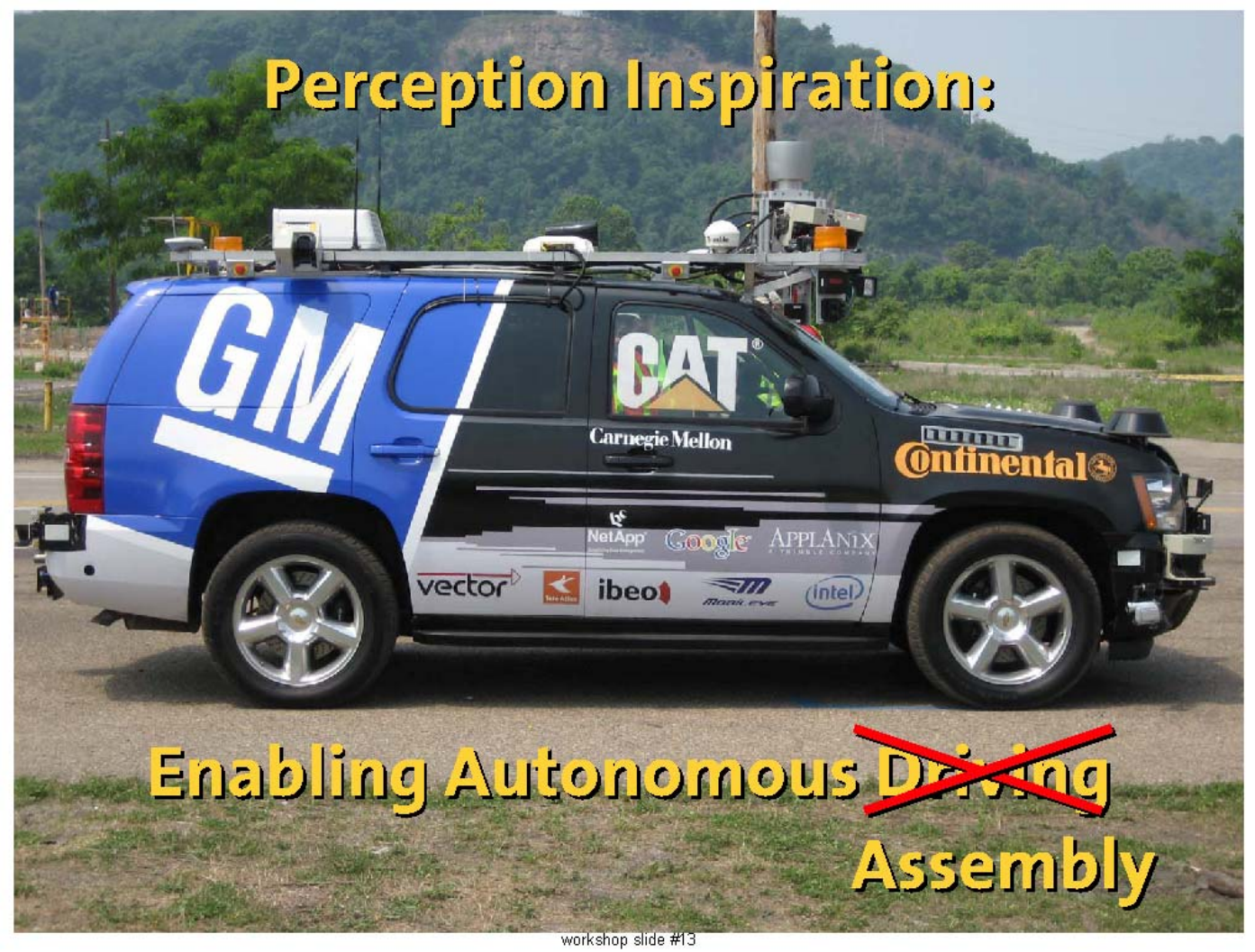

\section{Perception Inspiration:}

Sensors, Sensors, Sensors, ... Everywhere

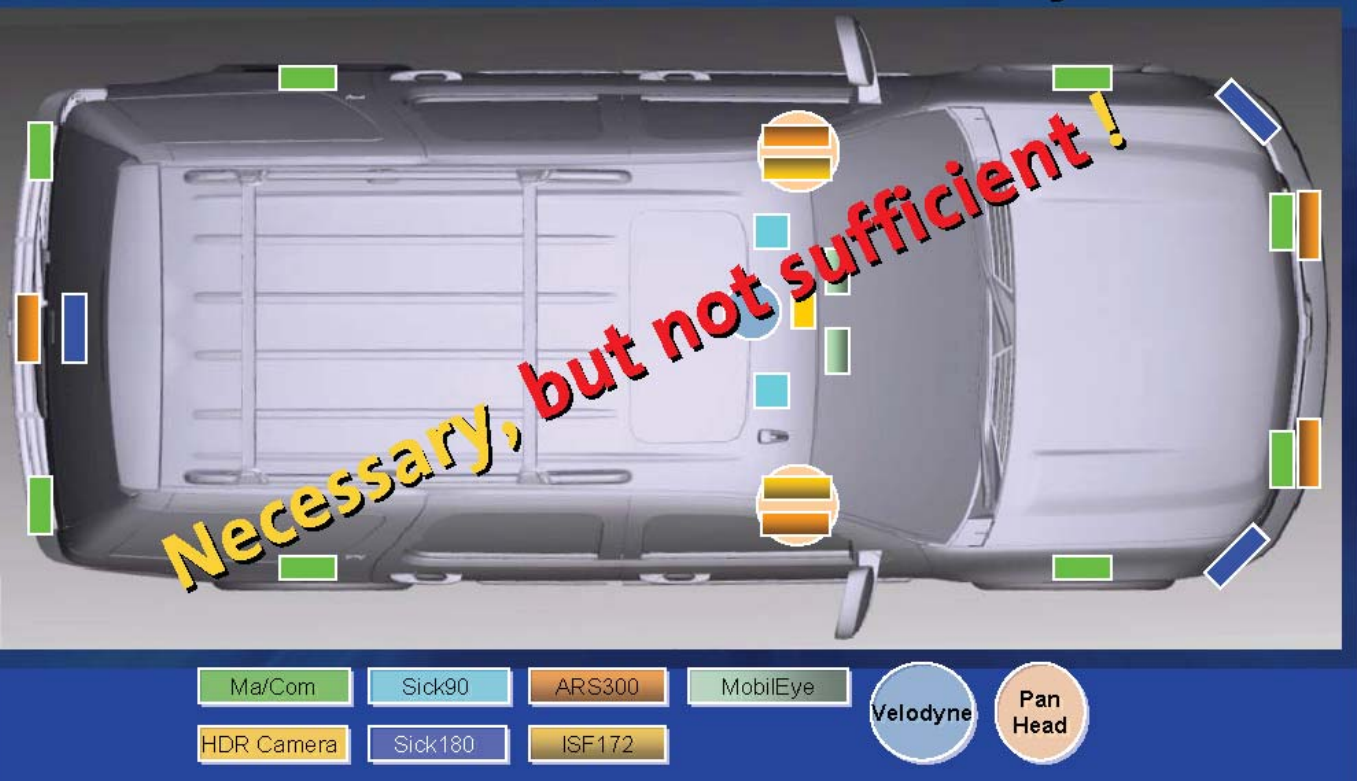

Workshop slide \#14 


\section{Perception Inspiration: Sensors Serve their Purposes}

On-road driving

Obstacle avoidance

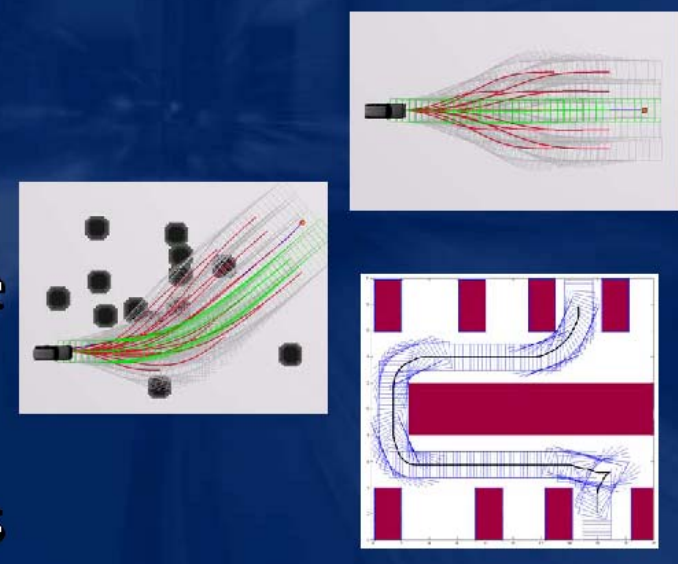

Parking maneuvers

\section{Autonomous System of High Capabilities}

Requires

Intelligent High Level Planning,

Robust and Adaptive Low Level Behavior Control

With Adequate Dynamic System Response

$$
\text { One Examples NIST'S 4D/RCS }
$$




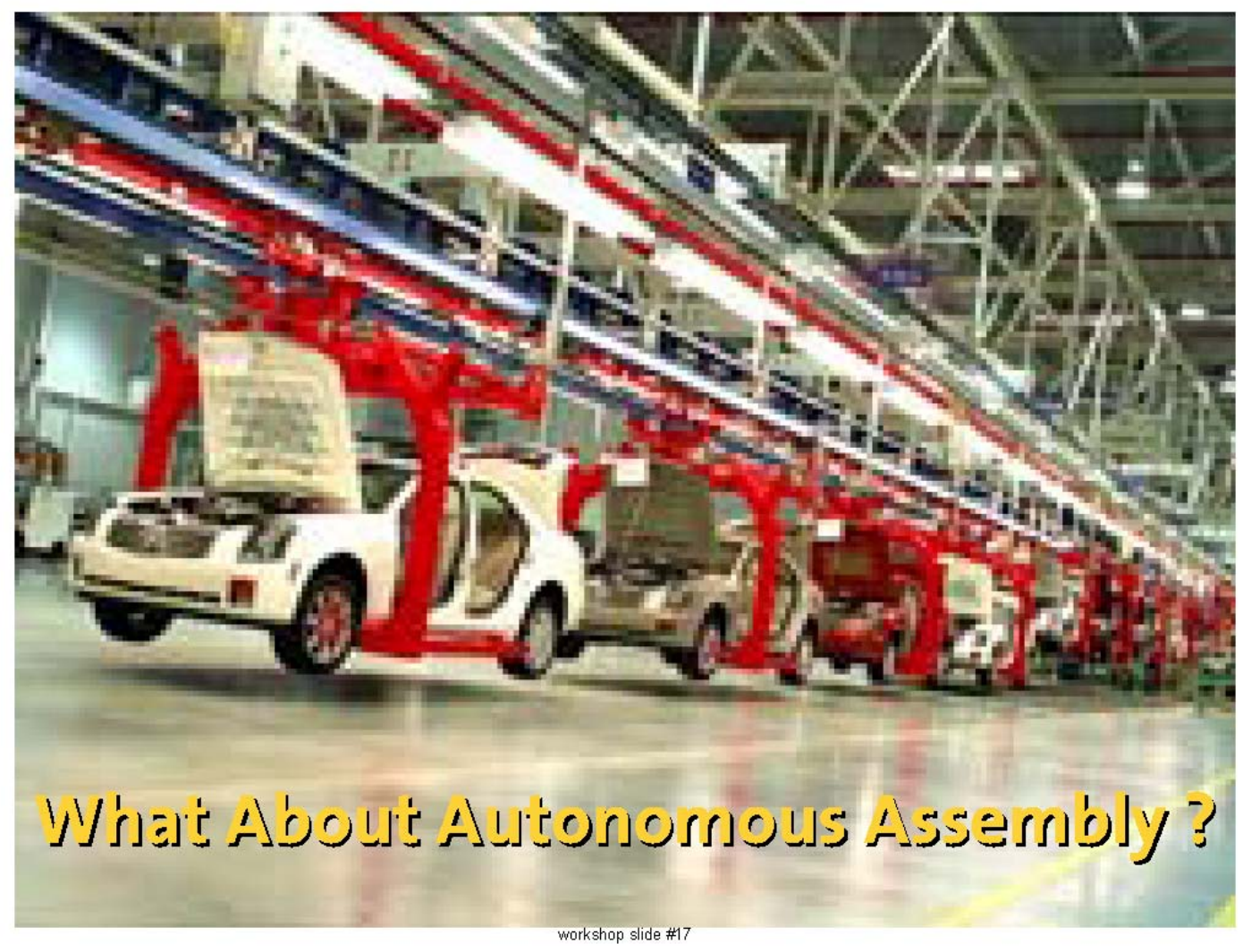

\section{Automotive Manufacturing}

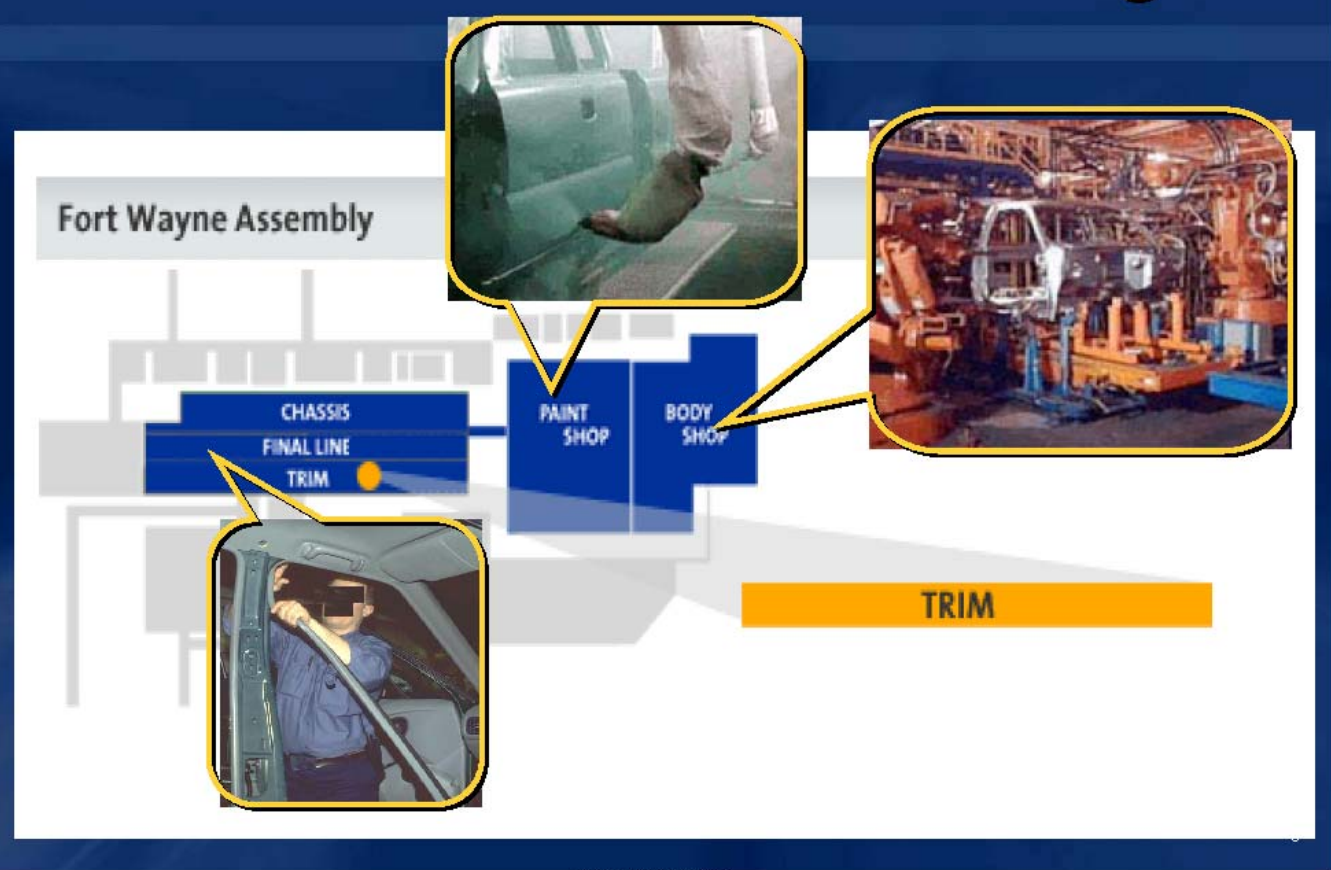

Workshop side \#18 


\section{Automotive General Assembly}

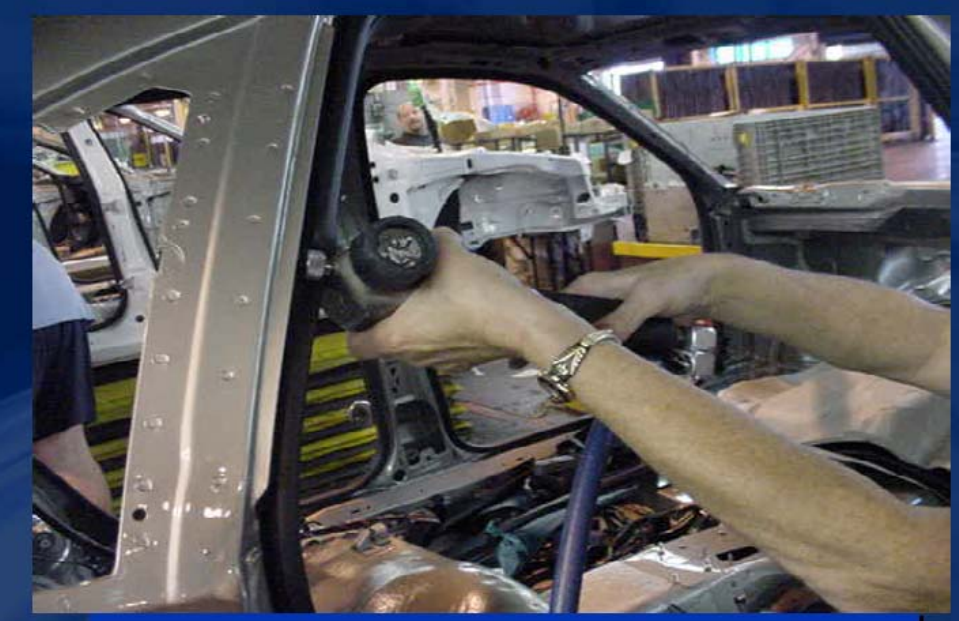

http://gmtv.feedroom.com/index.jsp?auto_band=x\&/ff=sv\&fr_story= b07964c0dc5936a75fc24bc5beb97169c55f390e

\section{Complexity of Assembly Tasks}

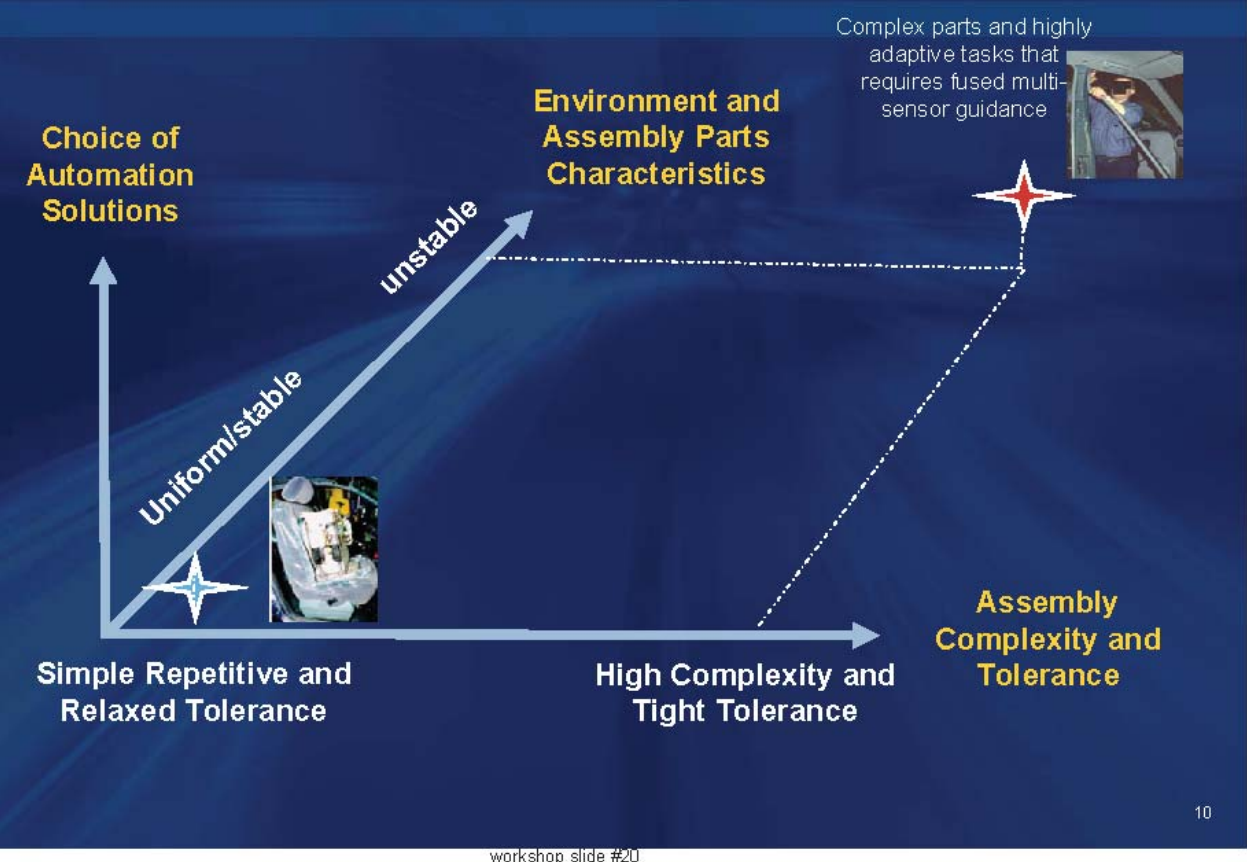

workshop side \#20 


\section{Automation of GA}

Complexity of GA tasks dictate the robotic automation solution:

- Intensive and pervasive sensing at right location and multiple locations

- Intelligent adaptation to parts and environmental variation - no bodyshop type precision locating mechanism available for automotive general assembly

- Coexist in the dynamic environment where operators perform the

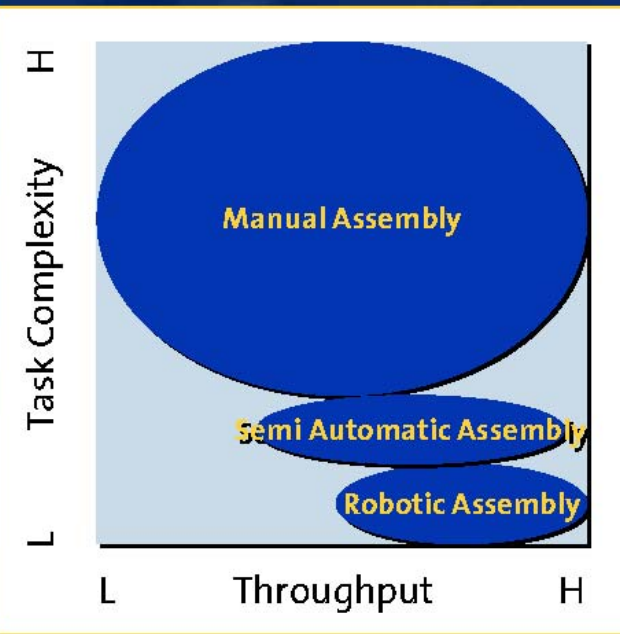
assembly task

\section{What About the Performance?}

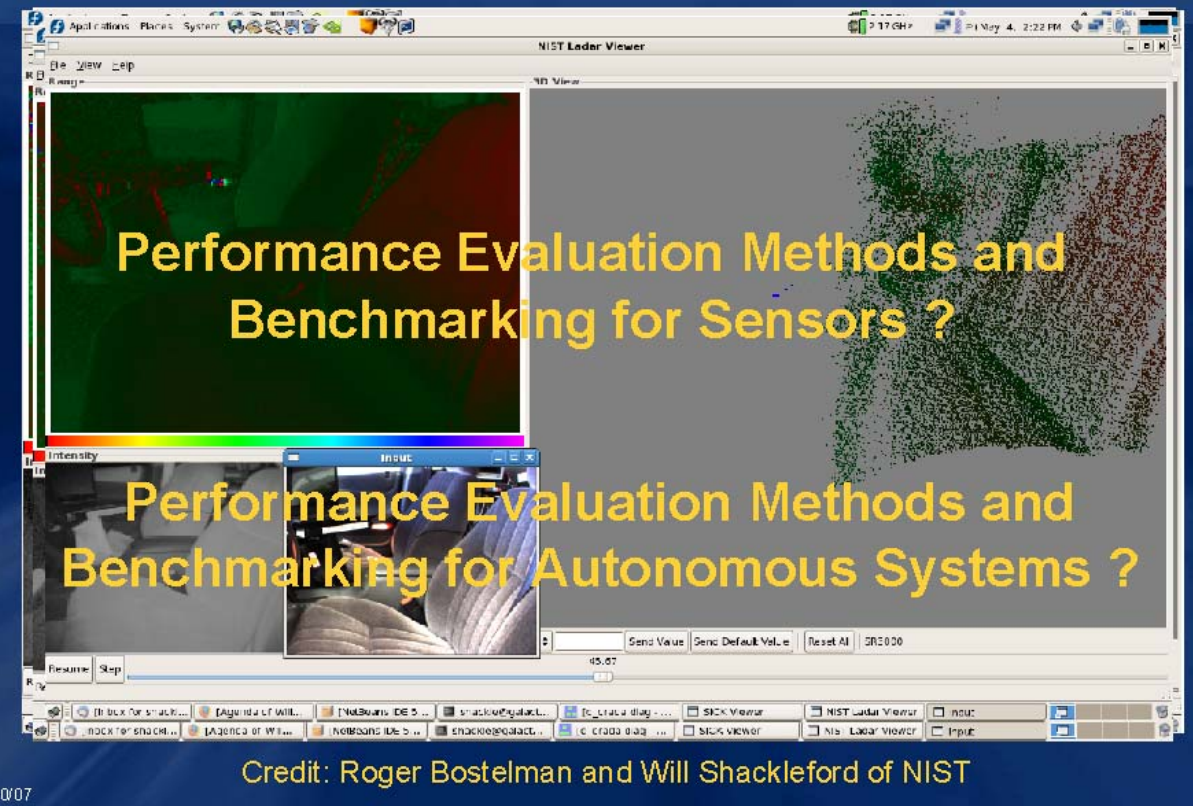

Workshop slide \#22 


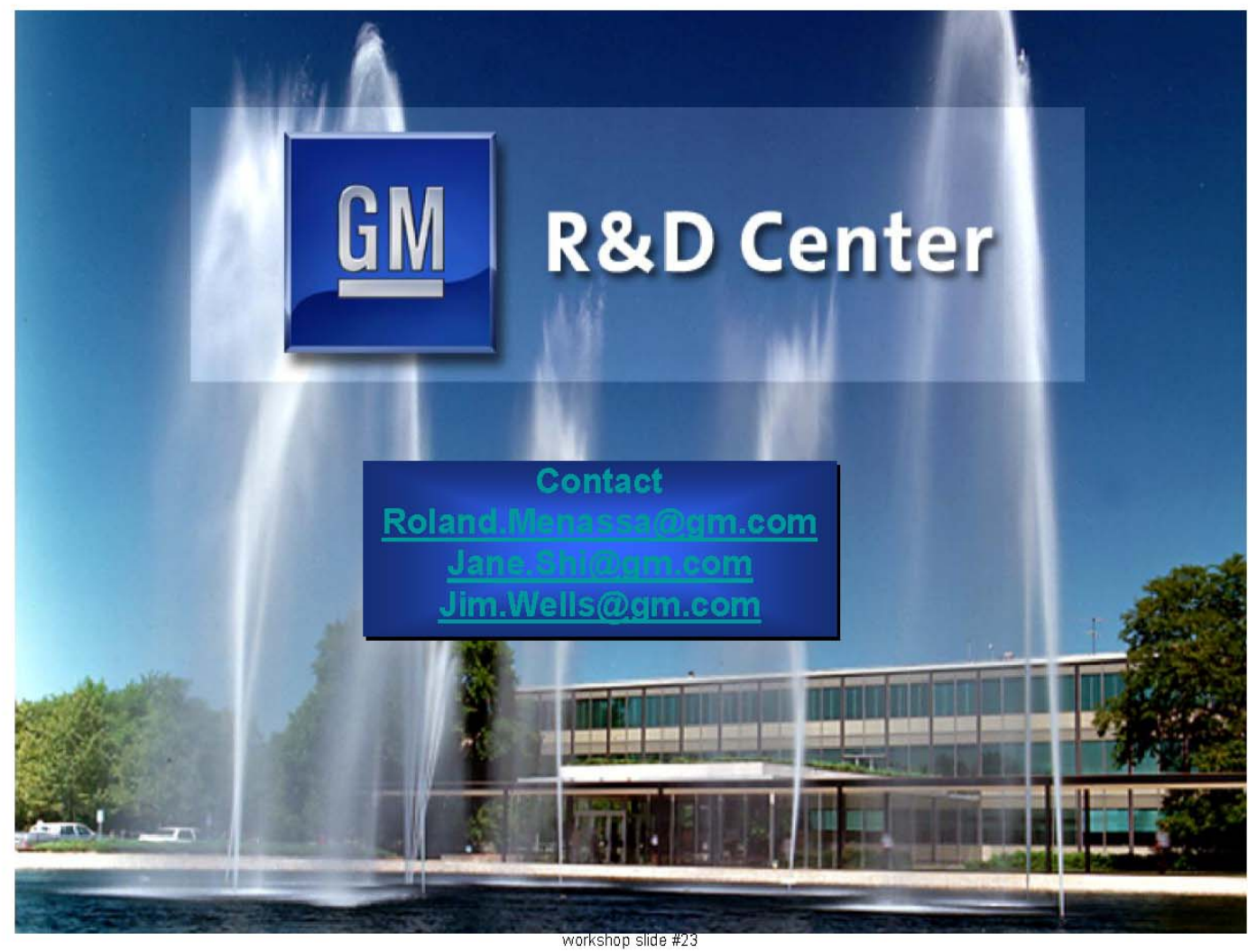




\title{
The Was $_{\text {ajuington }}$ jost
}

Automation Challenges

\author{
Conrad Rehill
}

Manager of Systems Engineering

workshop slide \#24

\section{Production Facilities}

- NW Washington, DC

- Newsroom \& Business Functions

- Springfield, Virginia

- Printing: DC, Virginia, National Edition

- Weekday pre-print advertising insert

- Sunday pre-print advertising collation

- College Park, Maryland

- Printing: Maryland \& North 


\section{Changing Business}

- The product: News \& Advertising

- Decline in newspaper readership nationwide

- Internct, Cable Telcvision ... Recycling (?)

- Increasing selectivity on advertisers part

- increased focus on targeted delivery of advertising with smaller zones / higher penetration

\section{Newsprint Delivery}

- FMC PICS system (installed 1998)

- Redundant control system

- 16 vehicles, 14 required to meet peak demand

- 150,000 kg Virginia / 100,000 kg Maryland per night 


\section{What affects reliability?}

- System

- controls

- vehicles

- Human factors

- Operators

- Maintenance
- Environment

- vibration / shock

- RF environment

\section{Vibration \& Shock}
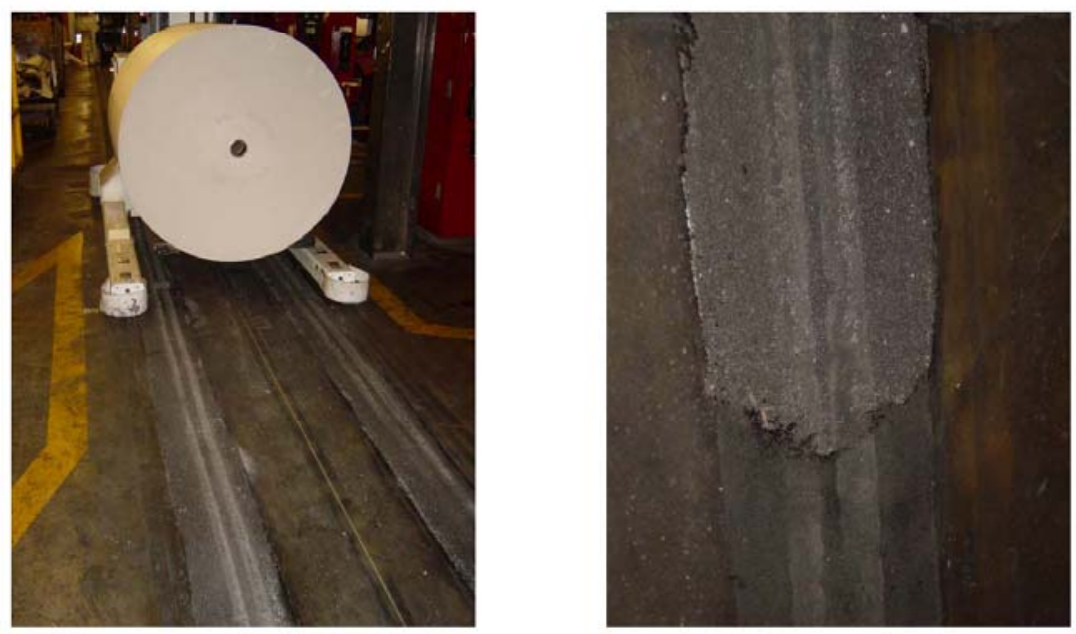


\section{Vibration \& Shock}

- Original condition of floor never met AGV vendor specifications

- flatness, incline, slab gap

- Continual replacement of vibration-worn vehicle electronics and suspension components

- The fix: cut new epoxy resin "lanes" in floor providing a seamless travel path

\section{Analyzing the Fix}

- New travel lanes required shot blasting 1" deep channels in existing floor

- Poor cleanup of shot material becomes embedded in vehicle wheels

- Shot-embedded wheels wear 3/4" grooves in areas of floor not yet repaired with epoxy resin 


\section{Analyzing the Fix}

- Excessive vibration while traversing worn areas of floor and shock of transitioning from worn to unworn levels increase vehicle breakdown rate

- Approach critical number of vehicles simultaneously down (14 of 16 required to meet daily peak demand)

\section{Continuous Monitoring}

- Use PC/104 board with standard WiFi

- onboard 12-bit A-D conversion

- single-axis accelerometer

- tap into vehicle onboard power

- RS-232 serial data tap to eavesdrop on hostvehicle communication: location

- dump data across WiFi at vehicle closest approach to data repository 


\section{Other Opportunities}

- Vision systems: color registration

- Replacement of existing system costly

- Maintenance of existing system questionable

- Use GNU tools with COTS PCI-based counter, $\mathrm{DIO}$, and frame grabber cards

- Vision systems: bundle tracking

- need to have knowledge of bundle identity prior to application of a label

\section{Color Registration}

- The task: identify the registration pattern within a field that may contain confusing data

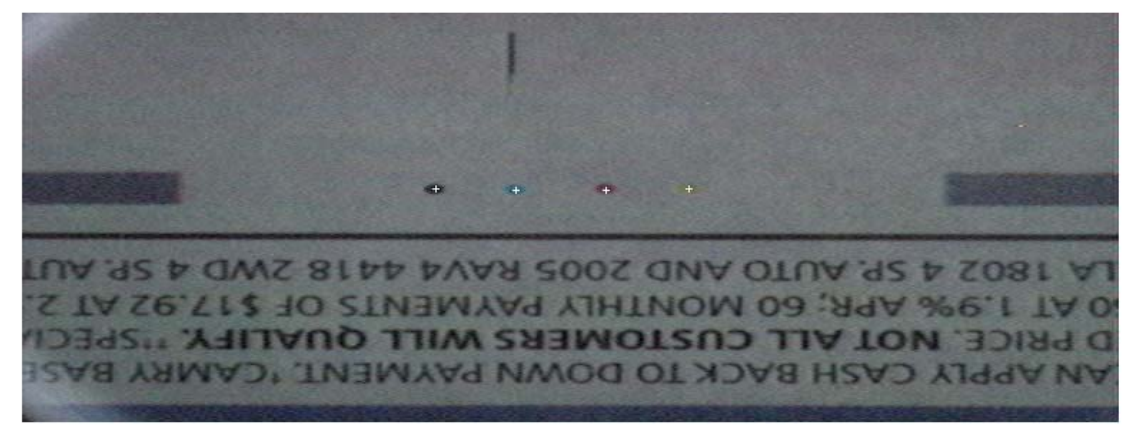




\section{THANK YOU!}

Questions?

workshop slide \#37 


\title{
Sensors On The Robotic Containerization System (RCS)
}

\author{
Engineering
}

Package and Material Handling Development

Joyce Guthrie

\section{RCS}

USPS has purchased and installed 167 systems

$>$ Each system contains 2 gantry style robots

$>$ USPS has decided to add 10 more RCS III systems to the fleet, bringing the total to 177 systems by 2009

$\square$ First RCS I system was installed in Santa Ana, CA in $\mathbf{2 0 0 0}$

$\square$ Last RCS II system was installed in St. Louis, MO in September 2007

$\square$ USPS is the largest user of Gantry robots in the world 


\section{RCS - Small Video's}
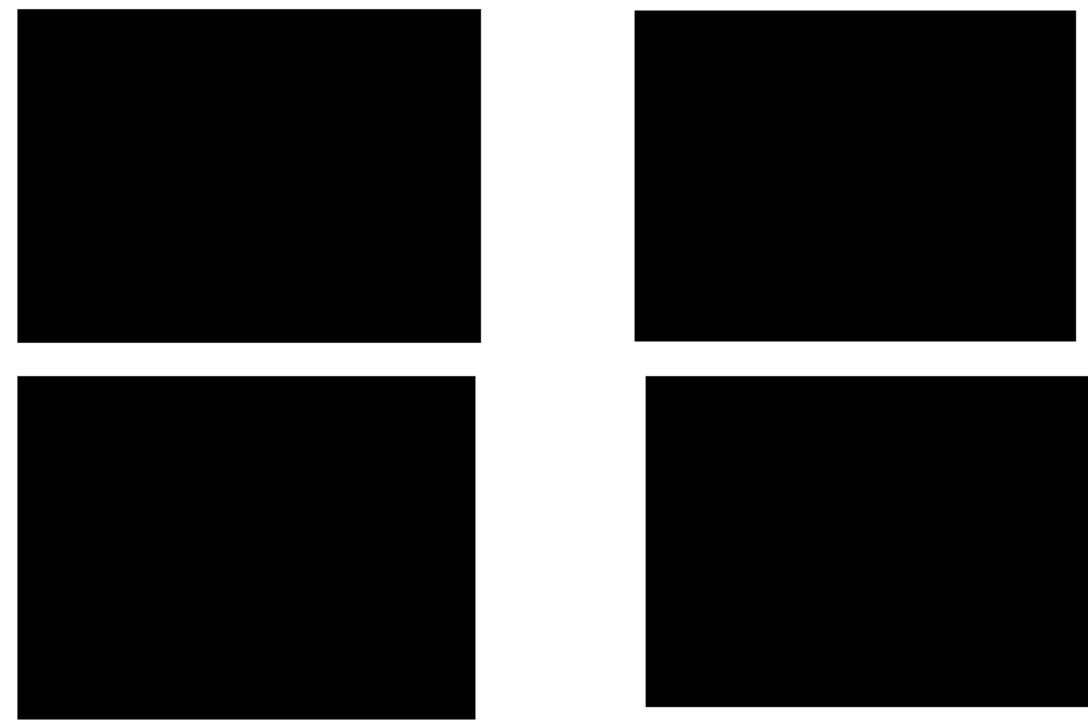

\section{RCS}

\section{Sensors}

We use a wide variety of sensing with the robotic system

- Collision sensing on the robot arm and EOAT (end of arm tool)

- This is a Applied Robotics sensor that is attached to the arm prior to the EOAT for detection of ay collisions in the sideways position

- Search sensor

- To detect if a shelf in the container is there

- Detection sensor

- Pallet/container detection (to detect what is in the position)

- Docking station for present and type

- SMM detection stand 


\section{RCS}

\section{Sensors (continued)}

- Safety sensing

- Levels of access control sensing

- $1^{\text {st }}$ level is physical door

- Door interlocks

- Light curtains

- On gantry pop up hard stops

- Switches to determine which zone the robot is in

- Pull cords

- Plexiglas doors with interlock switch at pick-up station

- Tray present sensing for zone control

RCS

$\square$ Sensors - Lesson Learned

Issues

- Dirt effects them

- Sensitivity range (if adjustable)

- Type of material on the retro-reflective (bounce back issue)

- Alignment sensitivity

- Obsolescence

- Connector configurations 


\section{Shipbuilding: Automation Issues}

Ken Fast, General Dynamic Electric Boat supplied as generic information 860-433-6432, kfast@ebmail.gdeb.com

Presented by:

Roger Bostelman, NIST

at the

Dynamic Measurement and Control for Autonomous Manufacturing Workshop

Oct. 10, 2007

workshop slide \#44

\section{Overview/outline}

Shipbuilding offers some unique challenges in manufacturing:

- large, heavy structures

- long build time

- single-item build

- limited repeat

- (limited indoor fabrication space)

- large range of operations/disciplines

Everything is custom build due to long build time.

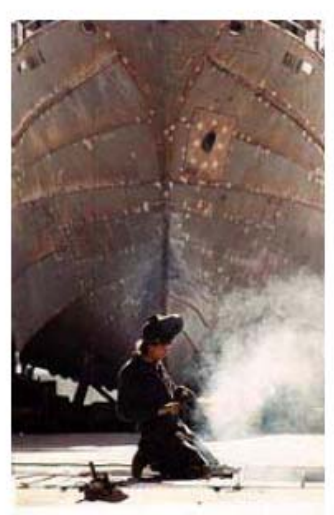

EUROP Sectorial Report on Industrial Robot Automation 


\section{Naval shipbuilding has some additional requirements}

- specialty materials - high strength steels, stainless, nickel/copper

- relatively tight tolerances

Probably costs more as a result

\section{Measurement tasks}

- piece/part verification

- flat cut plate - CNC cut now

- rolled/shaped plate

- assembly layout - not much fixturing

- assembly verification

- equipment mating holes/surfaces - in place!

- equipment/assembly installation, alignment

- large unit join alignment

- E.g., join two 40' x 40' x 100' weighing 100 tons 


\section{Unique Measurements}

\section{(at least for submarines)}

- circularity

- reference planes

- arbitrary references that may not be on the part

- E.g., 4' away from the part

\section{Automation - state of industry}

- all piece parts design-to-cut (CAM)

- mostly automated pipe bending

- some robotic welding

- some automated sheet metal cutting/forming

- waterjet, laser, oxy-fuel

- some automated plate forming 


\section{(non) Automation}

- permanent fixturing/workcells

- custom build, single item limits use of

- dedicated floor layout from unit to unit

- long build schedule, limited repeat, and large structures cause difficulty in maintaining

- automated material movement

- large, heavy items, outdoor transit, and changing layout limit use of

- automated material movement

- most material delivery requires lifting (sometimes heavy), safety issues limit

- automated welding/coatings application

- odd shapes, constrained spaces, material specifications limit use of

- (multi-pass welding, thick sections, pre-heating)

workshop slide \#50

\section{Design product model issues}

- limited manufacturing information in design

- limited repeat

- E.g., a lot of odd shapes (subs)

- 3rd party design

- may not have exactly what's needed on the drawing to make the part

- reference planes, odd shapes 


\section{Existing measurement techniques}

- part layout marking (laser etch)

- optical surveys

- photogrammetry

- laser alignment

- laser tracking

- "string" lines

- still works well!

\section{Current work efforts}

- part "families" - similar tasks/shapes with dedicated, but flexible, workstations

- (more) dedicated floor layout

- flexible, accurate location technologies - iGPS

- increased use of layout marking

- increased integration of manufacturing data with design product model

- robotic/automated welding

- robotic/automated coatings 


\section{Wish List}

- large area reference line/plane projection

- (a-la laser-level used in construction) - although, not visible in space, can't always measure to it from a tape measure

- multiple planes

- relative alignment (not level)

- solid steel interference

- line-of-sight issues

- automated welder

- field deployed, easy setup, easy program, multipass

\section{Thanks Ken!}




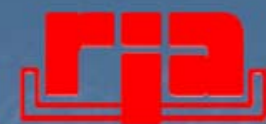

Robotics Industry Association

Lean - Next Generation Robots

Mike Calardo ABB Inc.

Robotics Division

Collaborative Robotics Adaptation

Brian McMorris

SICK Inc.

Market Manager - Robotics

worranop stide 758

\section{Lean Next Generation Robots}

\lrcorner Are these Next Generation Robots?

Are these Industrial Robots?
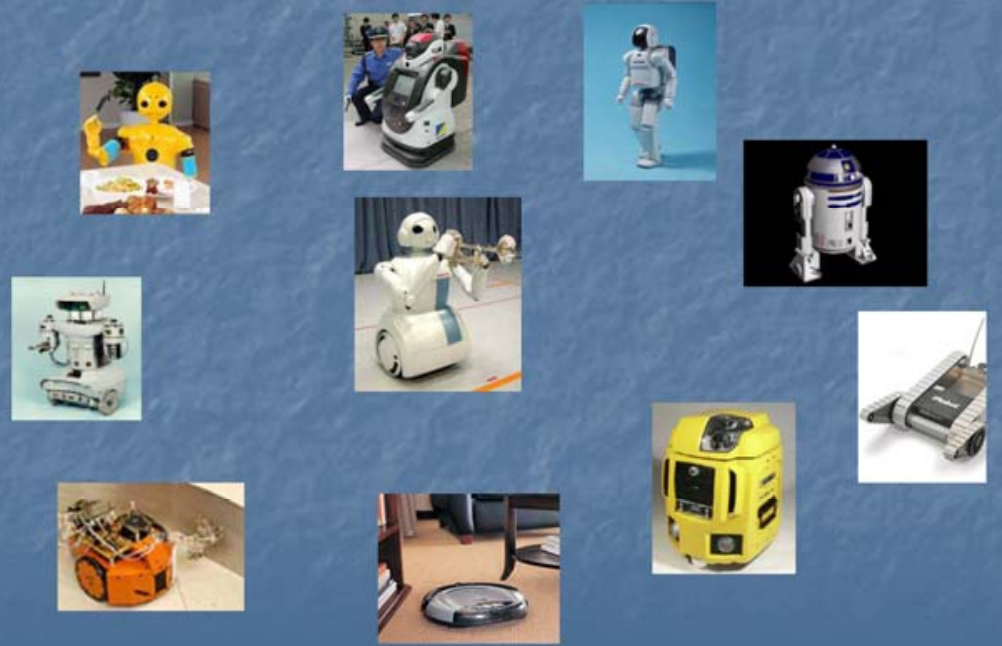

workshop slide "ISt 


\section{Next Generation Robots}

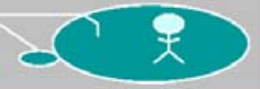

\section{\lrcorner Are these Industrial Robots?}
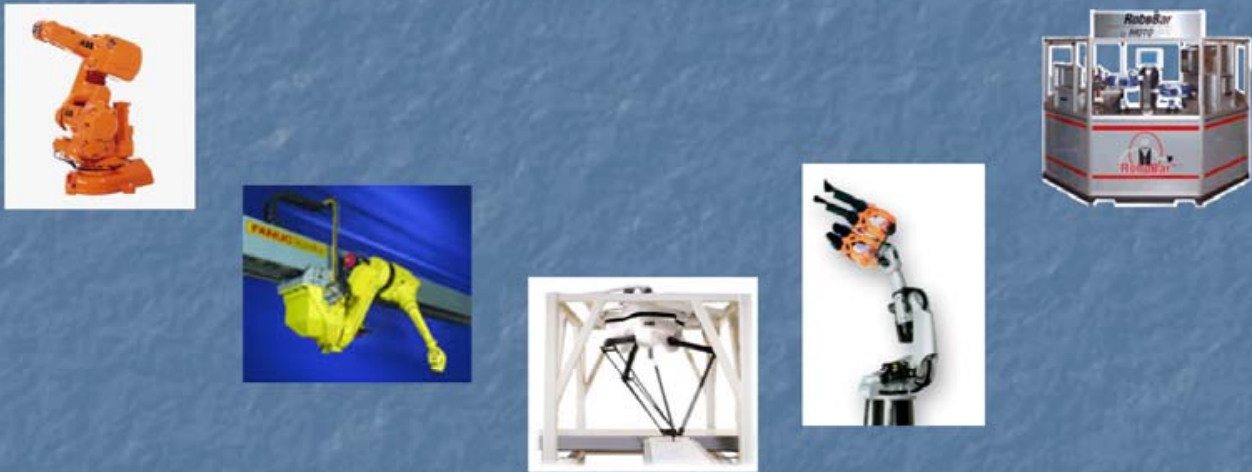

workstop silide 858

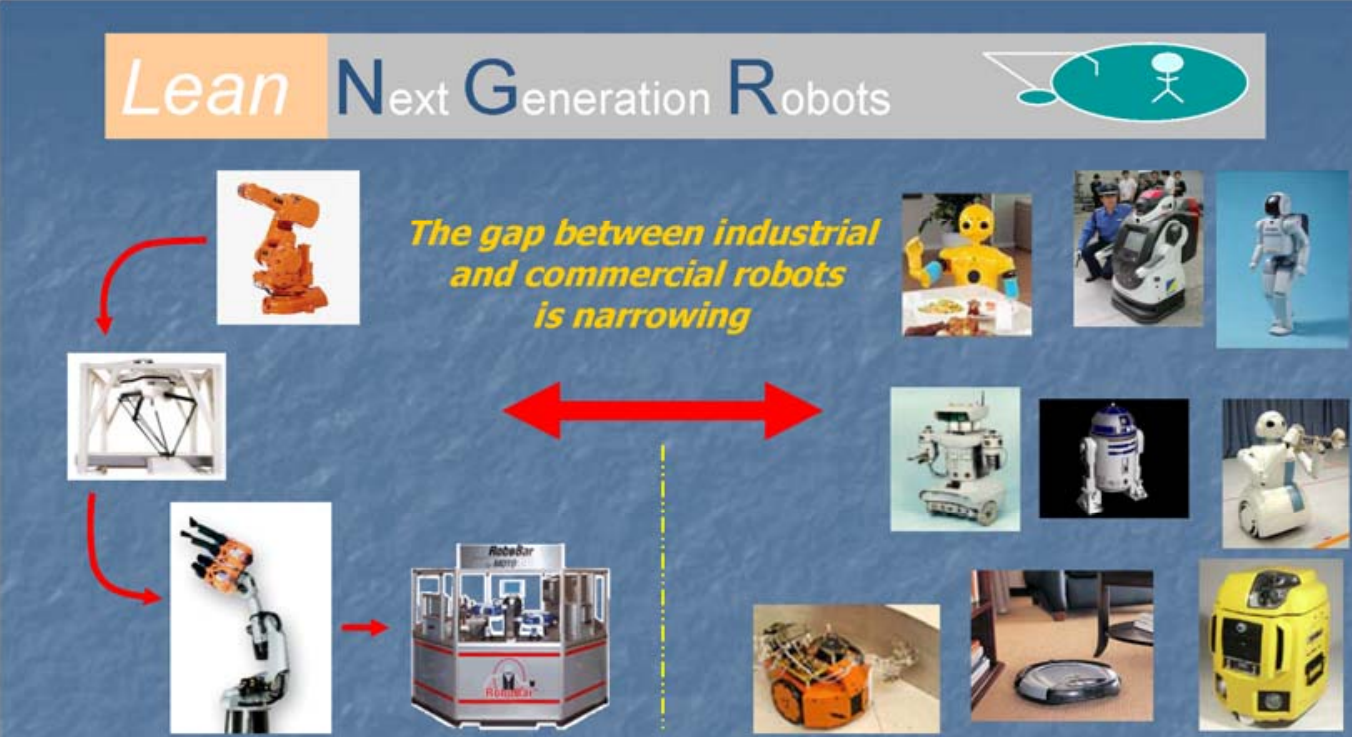

"The older industrial robotics segment, which was developed over the second half of the last century, consists of immobile, single task robots that have little interaction with humans or the world around them. They are termed industrial robots because they are exclusively employed in manufacturing and factory floor automation. You know the type. They are the robots, actually the articulated robotic arms, responsible for spot welding our cars, painting our refrigerators and checking for irregularities in assembled products."- Dan Kara President of Robotics Trends

Workshop side $\$ 59$ 


\section{Next $\mathbf{G}_{\text {eneration }} \mathrm{R}_{\text {obots }}$}

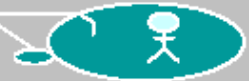

\section{What is a Jean Next Generation Robot?}

\lrcorner The main characteristic of lean Next Generation Robots is that they will be capable of working more closely with Humans

I ANSIRIA/SO 10218 Standards are in development to safiely enable this functionality which is called "Collaborative Operation"

\section{Lean Next Generation Robots} Which installation is safe?
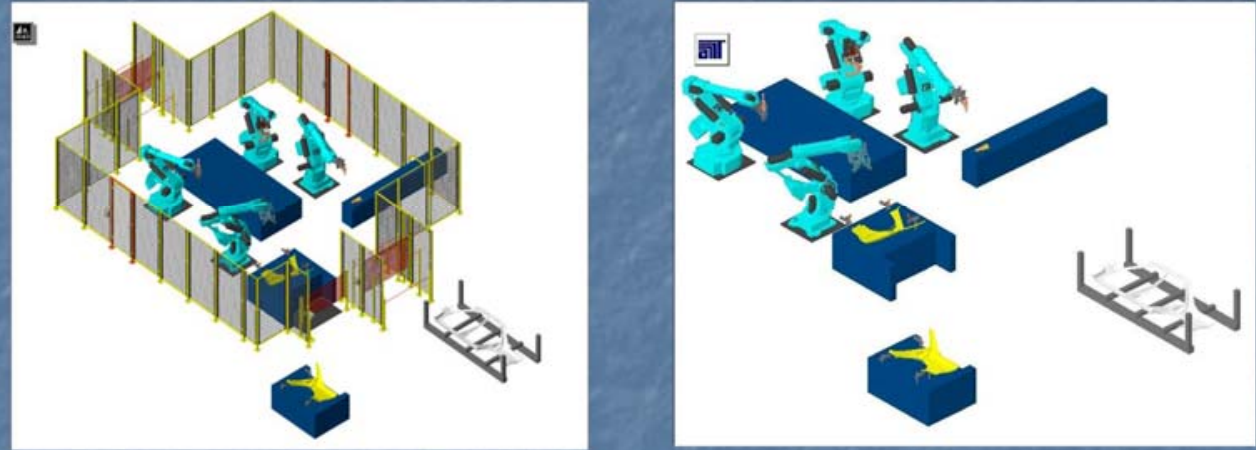

With future NGR technology, the answer may not necessarily be the one with the highest fence! 


\section{Next Generation Robots}

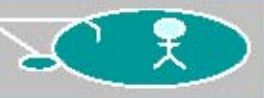

\section{Evolution of Industrial Robot Safety}

I Early 1980 's - No safety standards specific to robots

\lrcorner 1986 - First RIA 15.06 Safety Standard Published, revisions - 1992 \& 1999

I 2007 - ANSI/RIAMSO 10218-1 Adopted (August)

I However, as this level of safety has been raised, the amount of restrictions have increased. Basically the direction has been to prevent close human interaction with the robot whenever possible.

\lrcorner In these early years "safe" meant higher fences, more E-

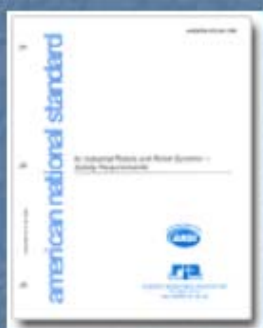
Stops and more ways to keep humans away from the automation. Increased clearances distances, more floor space required, more fencing, more complex and more costly.

I This trend is restrictive, very expensive and inefiicient!

Workstopsibe

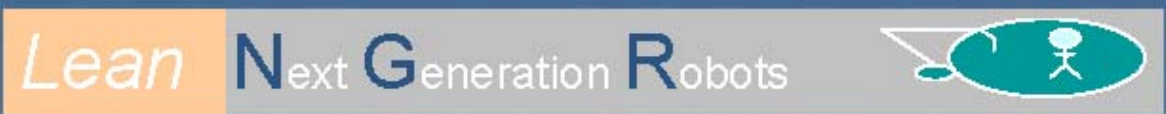

\lrcorner Safe meant stop (requiring a fault clearance and manual restart) whenever a human was too close; But, is this best for productivity?

\lrcorner Is it best to stop the robot with a binary decisjon just because someone is near it?

\lrcorner What if the robot slowed down when someone was close and stopped when they are too close and in danger?

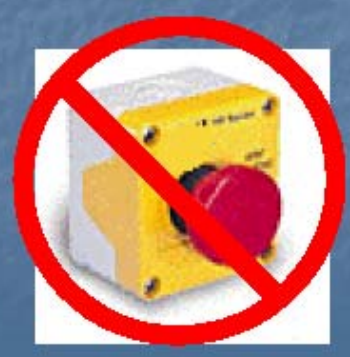




\section{Next Generation Robotis}

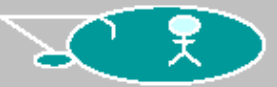

\section{NGR Technology}

\lrcorner In the next several years robot controllers will not only have control reliable, redundant stopping circuits; but they will also have control reliable feedback for axis position and motion is used as well.

I Software logic can be used for safety functions that were formerly limited only to hardware circuits.

\lrcorner Software logic can be used for saffety functions as long as it is protected from reprogramming, redundant and cross checked with multiple CPU monitoring. (This technology is used today in Safety rated PLCS)

\lrcorner Newly released (August 2007) ANSI/RIA/ISO 10218-1 standard defines Collaborative Operation Requirements in Clause 5.10

- 5.10.2 - The robot shall stop when a human is in the collaborative workspace...

I 5.10 .3 - When provide, hand guiding equipment shall be located close to the end-effector and shall be equipped with: a) an emergency stop and b) an enabling device...

- 5.10.4 - The robot shall maintain a separation distance from the operator (necessitating detection by a visual/optical means); This distance shall be in accordance with ISO 13855. Failure to maintain the separation shall result in a protective stop... 


\section{Next Generation Robots}

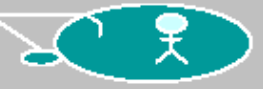

\section{Lean}

Lean means to eliminate waste. For robot cells and lines lean means:

- Decreased Floor Space

- Safer, yet lower cost saffety integration

$\checkmark$ Faster Integration/Startup Time

- More Flexibility

\lrcorner Lower operating cost

$\checkmark$ Increased uptime - High MTBF, Low MTTR

\section{Lean $\mathrm{N}_{\text {ext }} \mathbf{G}_{\text {eneration }} \mathbf{R}_{\text {obots }} \mathrm{C}$}

\section{Benefits of Lean NGR}

\lrcorner Decreased floor space

I Increased productivity

\lrcorner Reduced waste

I Reduced guarding

\lrcorner Zero clearance

\lrcorner Improved performance

Safety improvements that allow new applications je. collaboration with human activitjes 


\section{Next Generation Robots}

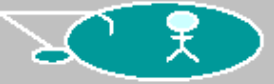

Drivers for NGR Performance Standard

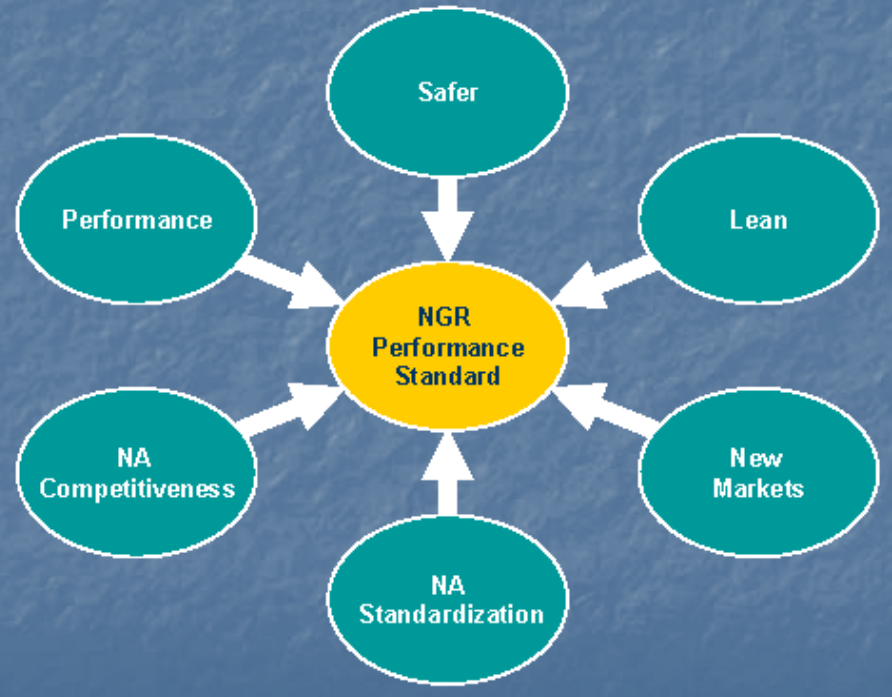

Workstopsibe

\section{Lean Next Generation Robots}

\section{Design considerations with NGR}

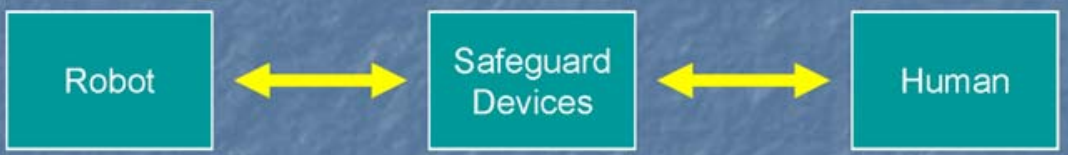

Size and Mass

Force Capability

Speed Capability

Reliability

Safety Rated Category to

connect safeguard

devices

Performance Criteria
Safety Rated Category Level

Type of device

Combinations of devices used

Cell layout considerations

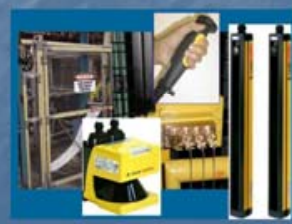

Proximity to machine

Frequency of Proximity

Level of Hazard Detection by the robot

Training and knowledge level of Human 


\section{Lean Next Generation Robots $D$}

\section{Examples of Lean NGR}

\lrcorner Replacement of mechanical switches on robot for safety zones
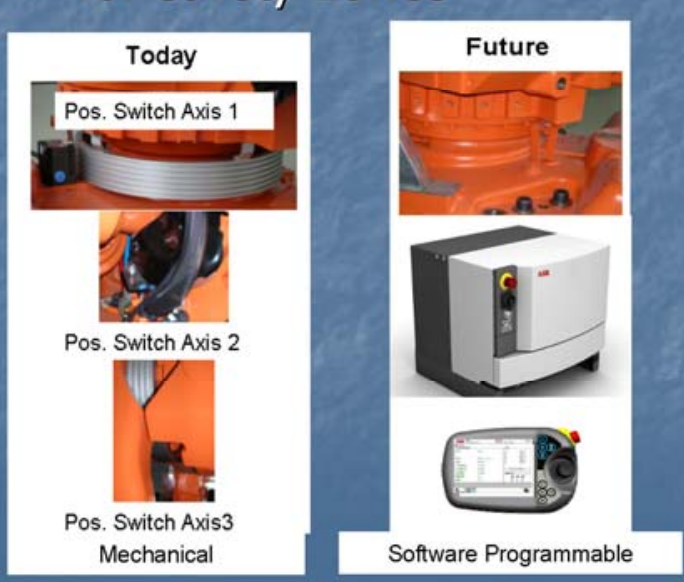

Eliminates mechanical axis switches

\lrcorner Benefits:

-Simpler setup

- Lower maintenance

-Higher MTBF

$\downarrow$ New Standard: ANSI/RIA/ISO 10218-1 Clause

5.12 - General Provisions

5.12.3: Safety-rated soft axis and space limiting

5.12.4: Dynamic limiting devices (vision / optical)

workshop side $\$ 70$

\section{Lean Next Generation Robots}

\section{Examples of Lean NGR}

\section{Safe Standstill}

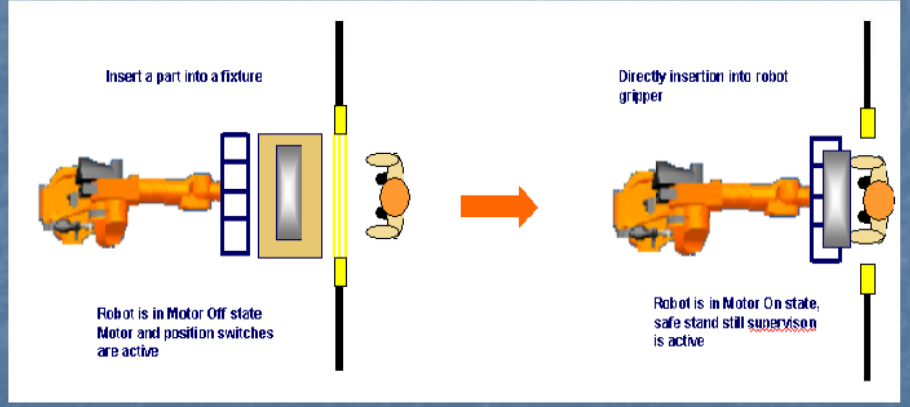

Benefits:

- Eliminate set down station

- No lost time to re-enable the robot drives after operator leaves the load zone 


\section{Lean Next Generation Robotis}

\section{Examples of Lean NGR}

\lrcorner Reduce speed if Human is near

\lrcorner Stop if Human is in danger

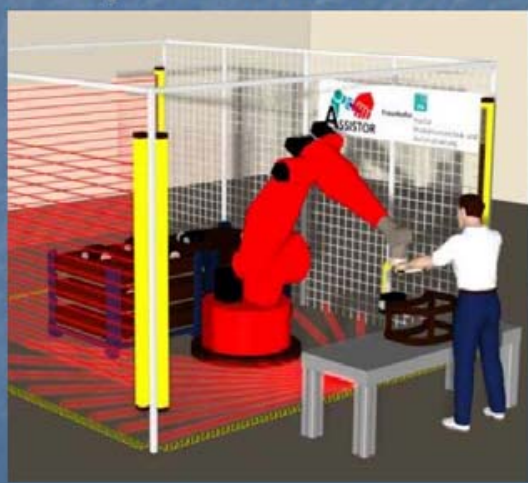

Benefits:

- Less lost time from accidental stoppage Smaller cell layout by lowering stopping distance of robot in operator area.

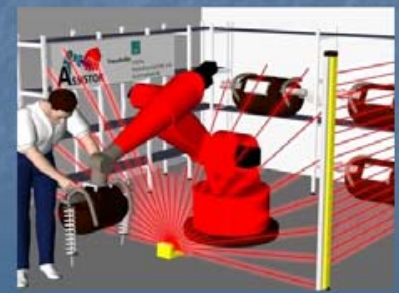

Morkshop sirde in/2

\section{Lean Next Generation Robots $C$}

\section{Examples of Lean NGR}
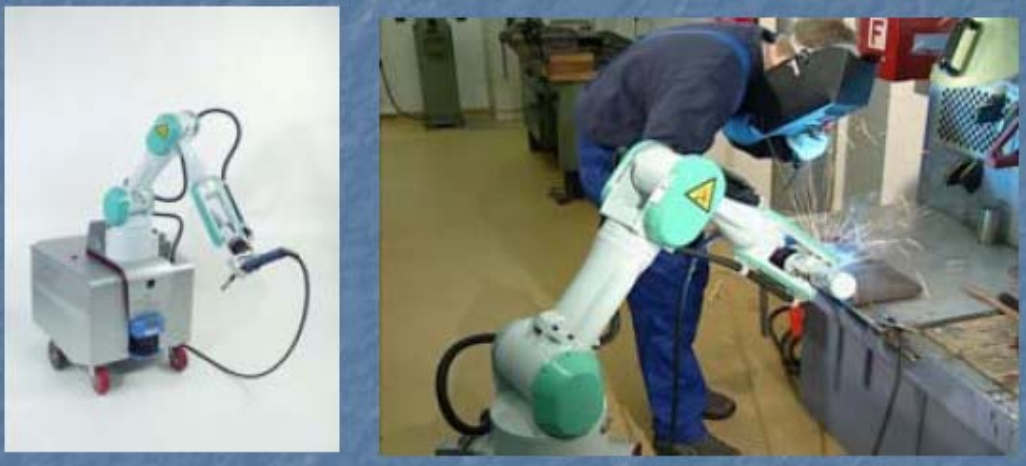


\section{Next Generation Robots $c D$}

Concept to categorize robots based on Safety Features

\begin{tabular}{|c|c|c|c|c|c|c|c|}
\hline & \multicolumn{3}{|c|}{ Jld Safeguards } & \multicolumn{4}{|c|}{ Human Proximity $S$ afeguards } \\
\hline housrial Robot Safety Category & 1 & 2 & 3 & 4 & 5 & 6 & 7 \\
\hline Emergency Stop & $x$ & & & & & & \\
\hline $\begin{array}{l}\text { Stopping Circuit } \\
\text { Brakes } \\
\text { Adustable Hard Stops } \\
\text { Brake Release }\end{array}$ & & $\begin{array}{l}x \\
x \\
x \\
x\end{array}$ & & & & & \\
\hline Hold to Run Switch & & $x$ & & & & & \\
\hline $\begin{array}{l}\text { Slow Speed Teach } \\
\text { Enabling Device }\end{array}$ & & $x$ & $x$ & & & & \\
\hline $\begin{array}{l}\text { Control Relabile Stopping Circuits } \\
\text { Control Relabile Axis Range switches (DLD) }\end{array}$ & & & $\begin{array}{l}x \\
x\end{array}$ & & & & \\
\hline $\begin{array}{l}\text { Redundant Feedback(RF) Range limit } \\
\text { Redundant Motion detection c apabiltiy } \\
\text { Proximity Slow Speed Auto Mode }\end{array}$ & & & & $\begin{array}{l}\bar{X} \\
x \\
x\end{array}$ & & & \\
\hline Safe lio / Field Bus & & & & & $x$ & & \\
\hline $\begin{array}{l}\text { Auto Mode Safe Speed Limit (RF) } \\
\text { Auto Mode Safe AcceliDecel Limit (RF) } \\
\text { Lowered Torque limits }\end{array}$ & & & & & & $\begin{array}{l}x \\
x \\
x\end{array}$ & \\
\hline $\begin{array}{l}\text { Human proximity Detection } \\
\text { Human Position avoidance }\end{array}$ & & & & & & & $\begin{array}{l}x \\
x\end{array}$ \\
\hline
\end{tabular}

Workstopslise the

\section{Lean Next Generation Robots - ?}

\section{Conclusjions}

\lrcorner NGR technology is in our future

\lrcorner NGR technology has promise to be safer and leaner than present technology

\lrcorner Performance and Safety Standards will need to be written to accommodate the new technology

\lrcorner Robotics industry manufacturers, integrators and users will need to understand the capabilitjes 


\section{Next $G_{\text {eneration }} R_{\text {obots }}$}

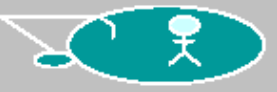

RIA actions

\lrcorner Work Group formed to begin discussing a framework of Lean NGR Performance standard.

\lrcorner Awareness presentations to stimulate interest and acceptance within working groups representing manufacturers \& users. RIA, AIAG, USCAR etc. 


\title{
Mobile Manipulation: Going beyond AGVs
}

\author{
George Kantor \\ kantor@cmu.edu \\ The Robotics Institute, \\ Carnegie Mellon Univeristy \\ in collaboration with: \\ Sanjiv Singh \\ Seth Koterba \\ Brad Hamner \\ D.H. Shin \\ M. Hwangbo
}

Dynamic Measurement and

Control for Autonomous

Manufacturing Workshop

10 October 2007

\section{AGV Overview}

- Automated Guided Vehicles (AGVs) follow prespecified guidepaths

- Wide range of material handling applications

- Many vehicles forms (forked, tow, loader)

- Many manufacturers (AGV, FMC, Savant, Webb, Egemin, $\mathrm{COH}$ )

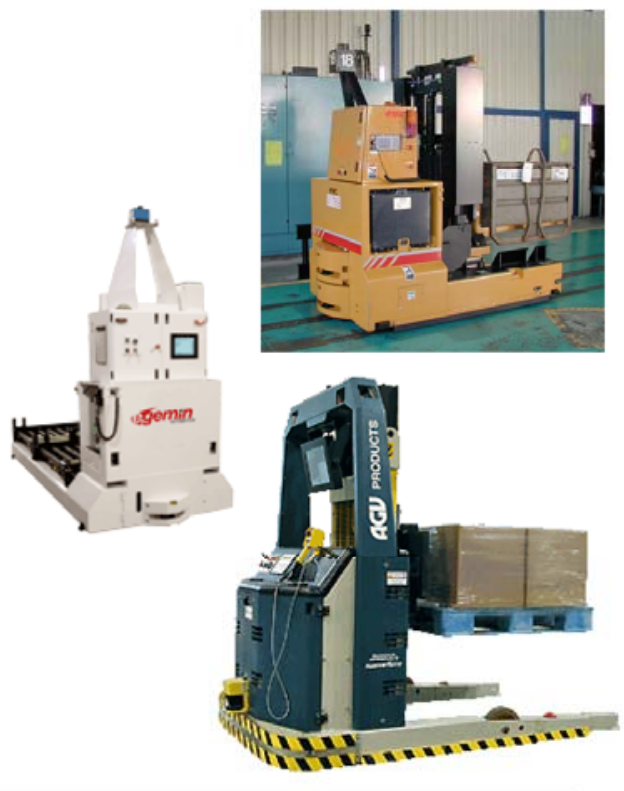




\section{Key Issue: Localization}

- Wired -- guidepath defined by wires embedded in floor

- Inertial -- localization from magnetic beacons embedded in floor

- Laser -- localization using laser scanner and reflectors

- Visual -- localization using on-board cameras (e.g., SEEGrid)

\section{Manipulator Overview}

- Robot arms with fixed workcells

- Many applications

- Over 30 years of commercial success

- Many manufacturers (ABB, FANUC, KUKA, Denso, Unimation, many others)
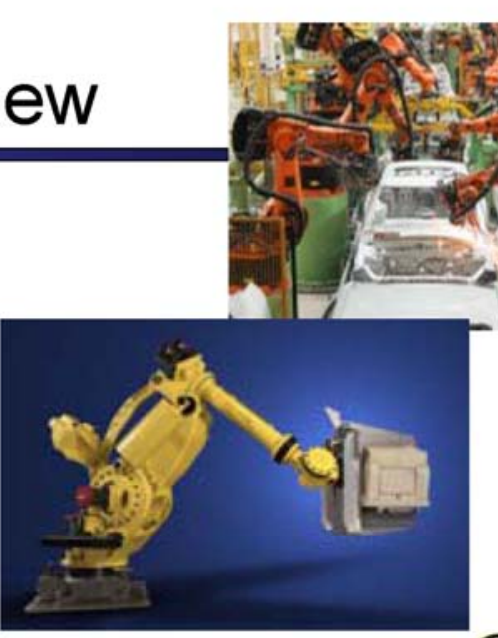


\section{Key Issue: Manipulability}

- Manipulability affects:

- Accuracy

- Strength

- Speed

- Is a function of configuration

- Must be considered when planning tasks

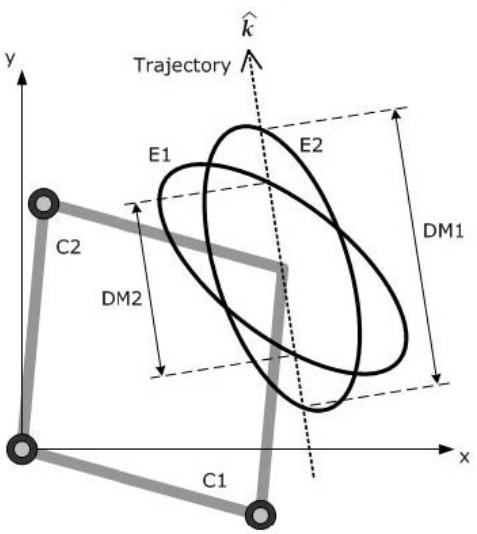

\section{Mobile Manipulation}

- Large workcell size

- Reduced infrastructure

- Flexible manufacturing
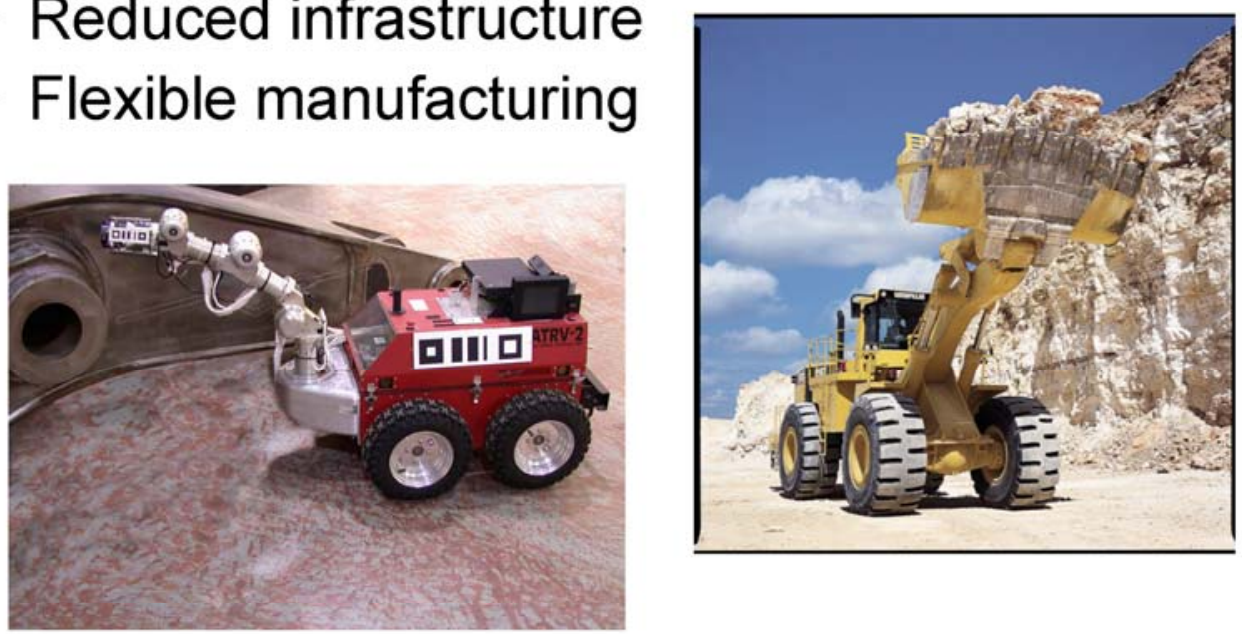


\section{Key Issue: Accuracy}

\section{accuracy $=$ localization + manipulability}

\section{Related Work}

- Coordination of locomotion and manipulation

- Redundancy optimization: Carriker

- Maximizing manipulability: Yamamoto

- Compensation of the dynamic interaction of the base and the manipulator:

- Tip over: Huang and Sugano

- Vehicle suspension: Hootsmans

- Cooperation of multiple mobile manipulators

- Derived from the force control methodology

- Control execution (RMRC for mobile manipulator)

\section{A First Approach}

\section{Selecting base poses:}

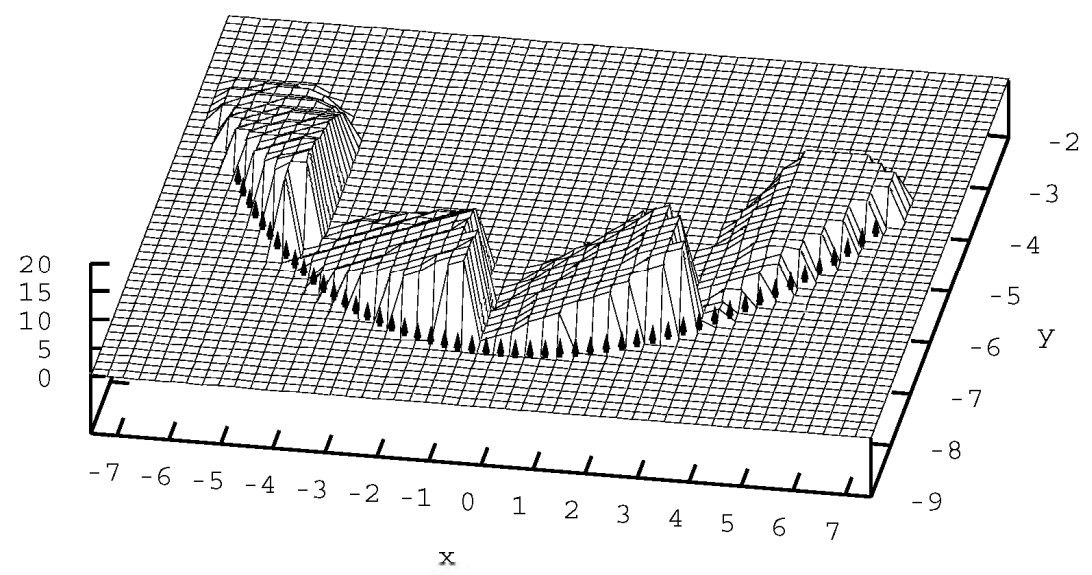

Each grid cell gets a score based on how much of the path and how well the it can be covered with the base at that point. 


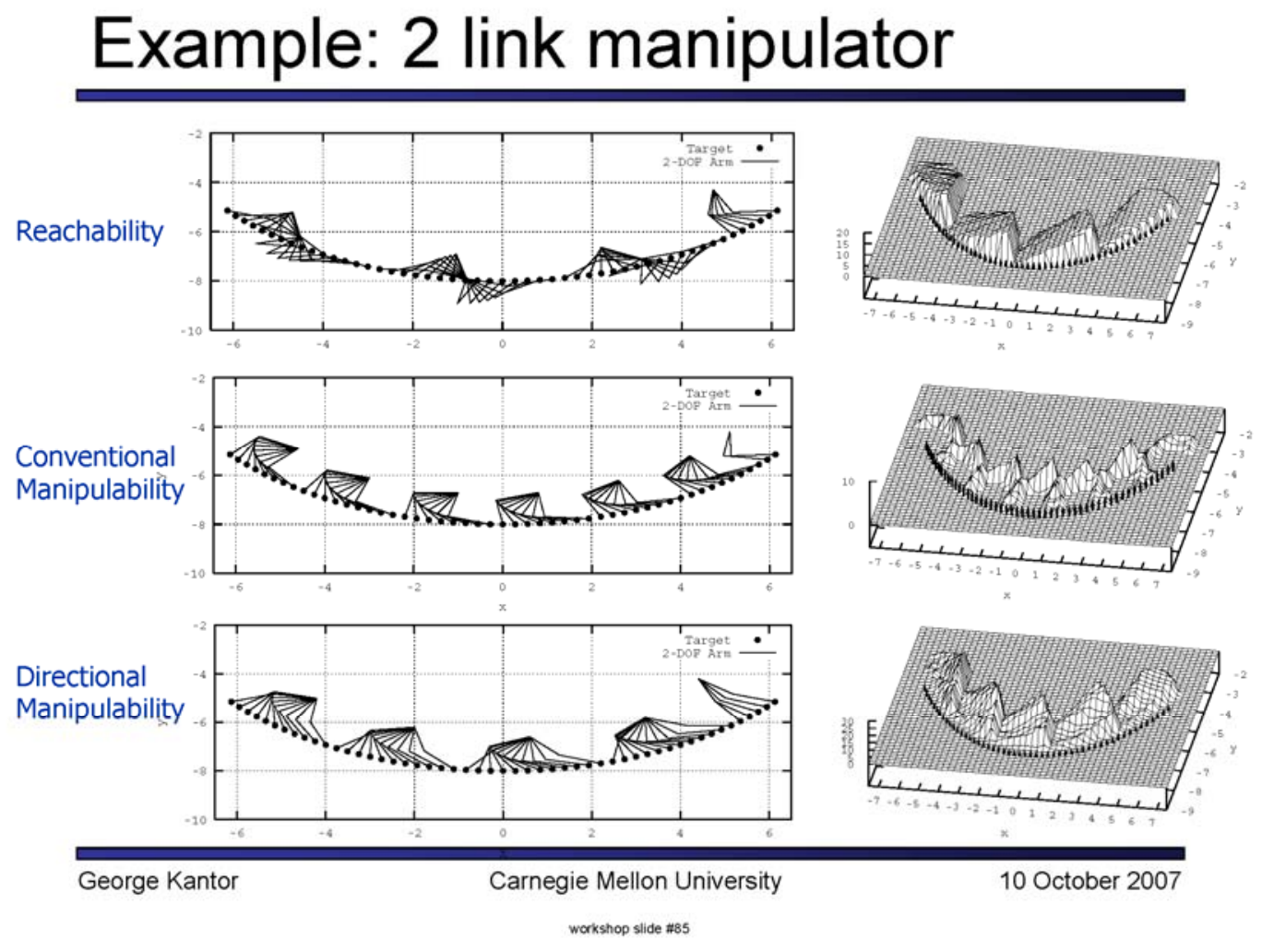

\section{Experimental Results}

QuickTime $^{\mathrm{TM}}$ and a

needed to see this picture. 


\section{Current/Future Direction}

\section{Integrated base/manipulator motion}

QuickTime $^{\mathrm{TM}}$ and a

Cinepak decompressor

are needed to see this picture.

\section{A Different Direction for AGVs}

QuickTime TW and a

Cinepak decompressor
are needed to see this picture. 


\title{
A Different Direction for AGVs
}

\author{
QuickTime $^{\mathrm{TM}}$ and a \\ Motion JPEG OpenDML decompressor \\ are needed to see this picture.
}




\section{Dynamic Visual Servoing}

Avi Kak

Johnny Park

German Holguin

Robot Vision Lab

Purdue University

\section{PURDUE \\ U N I V E R S I T Y}

morks

\section{PURDUE}

Purdue Robot Vision Lab

\section{Pundue Robot Vision Lab}

$\square$ Founded in 1978

$\square$ Performs state-of-the-art research in sensory intelligence for the machines of the future

口 Produced 39 Ph.D.'s so far 


\section{PURDUE}

\section{Significant Industrial Collaborations}

$\square$ Olympus Corporation (2004 present)

$\square$ Infosys Technologies (2007 present)

$\square$ Ford Motor Company (1994 2006)

口 Mitsubishi Heavy Industries (1992 1993)

口 Denso Corporation (1991 1995)

$\square$ Hitachi Heavy Industries (1991 1997)

Relevant Research Projects

$\square$ 3D Object Recognition and Bin Picking

$\square$ Vision-Guided Mobile Robot Navigation

$\square$ 3D Modeling

$\square$ Real-Time Background Subtraction in Video Imagery

$\square$ Distributed Sensor Networks

$\square$ Line Tracking for Assembly On-the-fly 


\section{PURDUE}

Line Tracking for Assembly-on-the-fly

$\square$ Goal:

- Develop a vision-guided robotic system that can operate on a moving assembly line

- Replace "stop-stations" in the assembly line

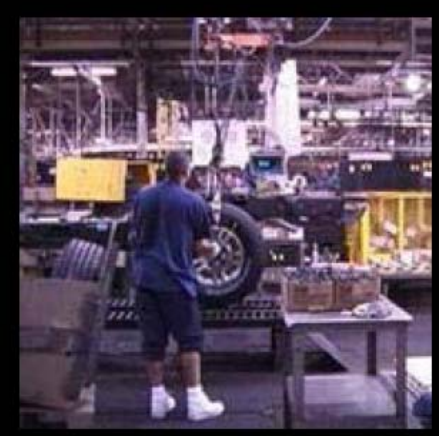

\section{Line Tracking System (video)}

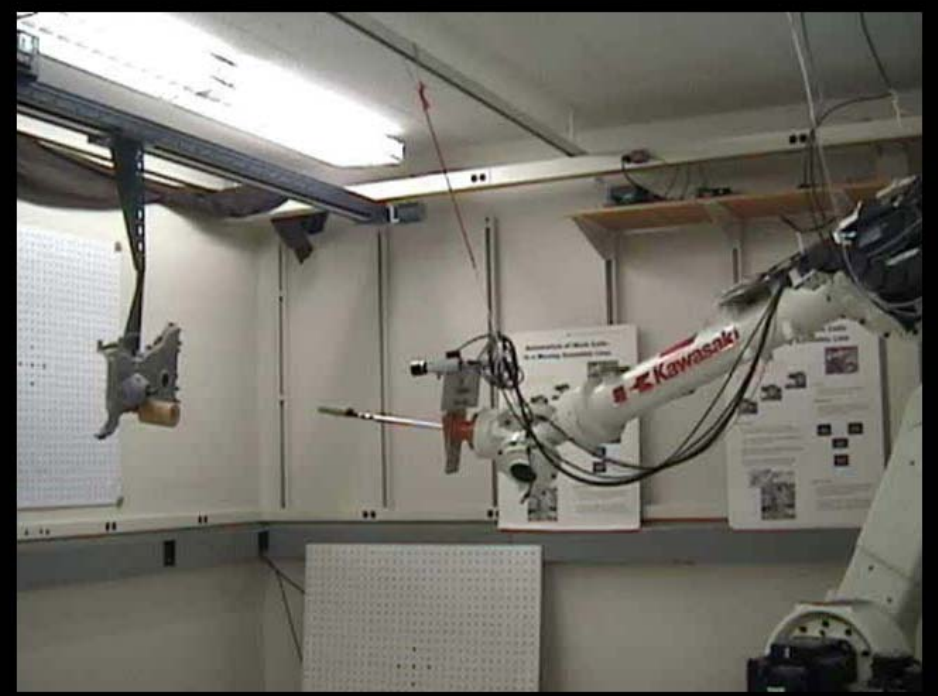




\section{System Overview}

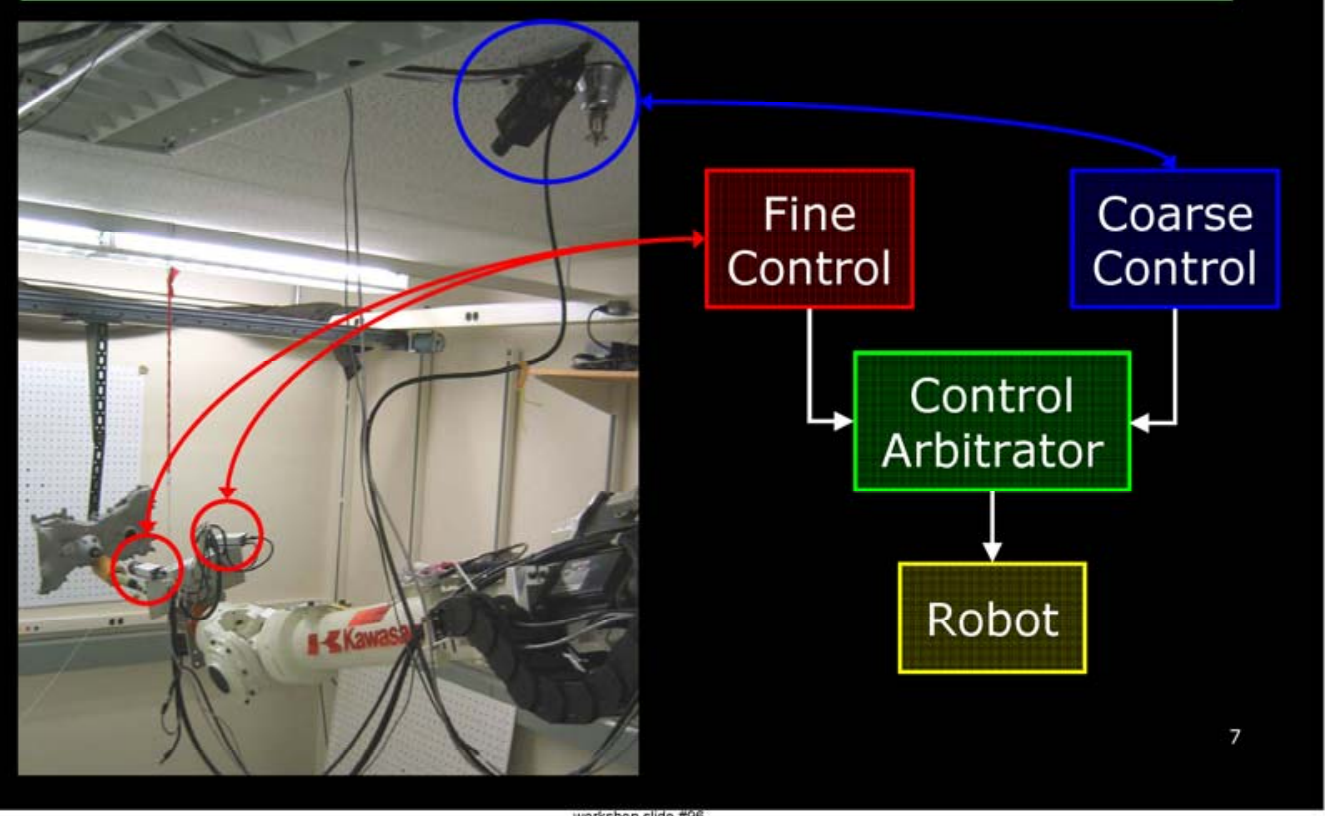

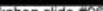

\section{PURDUE}

\section{Course Control}

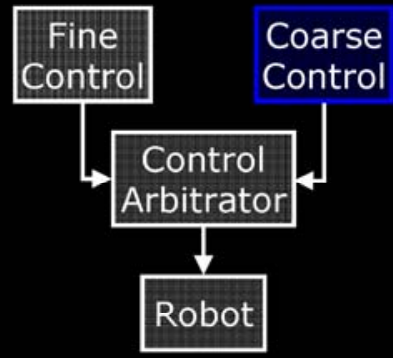

$\square$ Computes an approximate location of the target object in its field of view

$\square$ Executes the initial approach of the robot towards the target object 


\section{Coarse Control (video)}

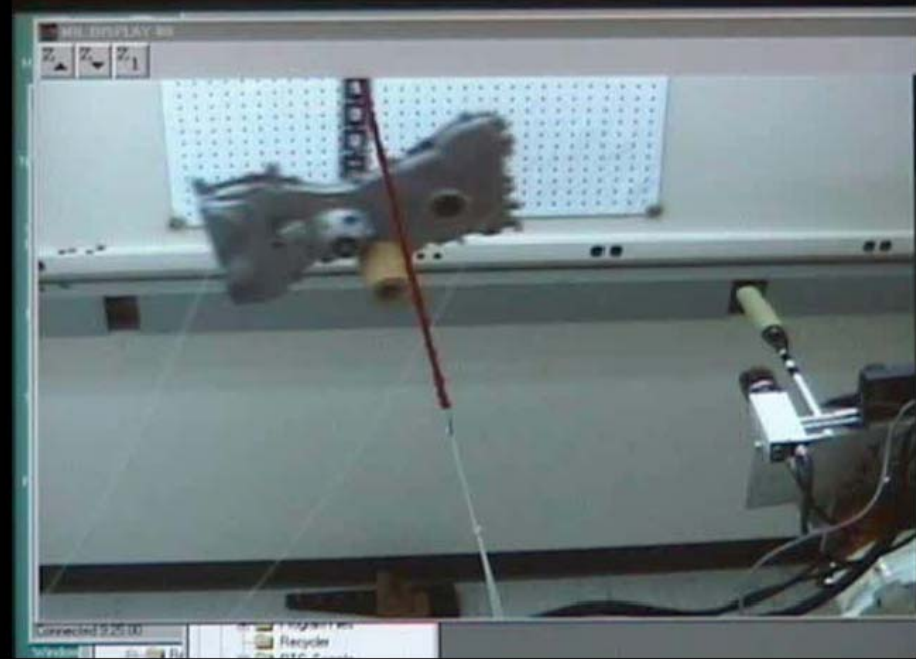

Fine Control
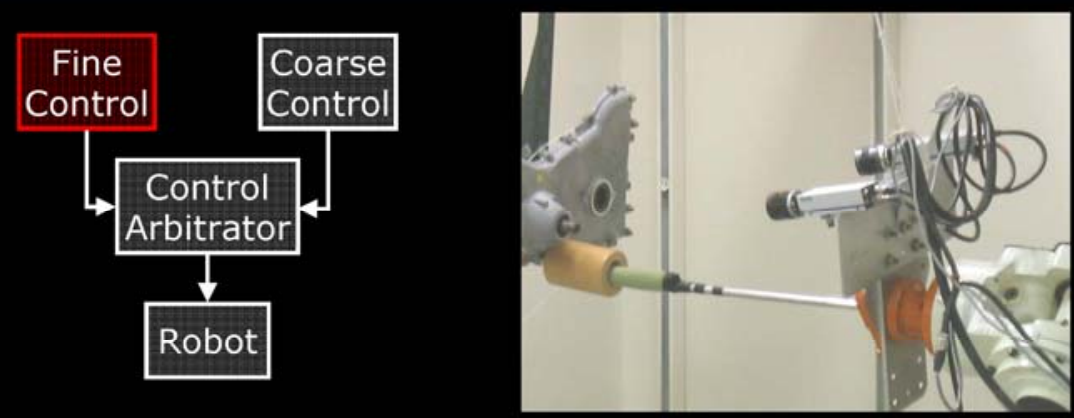

$\square$ Computes precise position and orientation of the target object to perform a specific assembly task 


\section{Fine Control (video)}

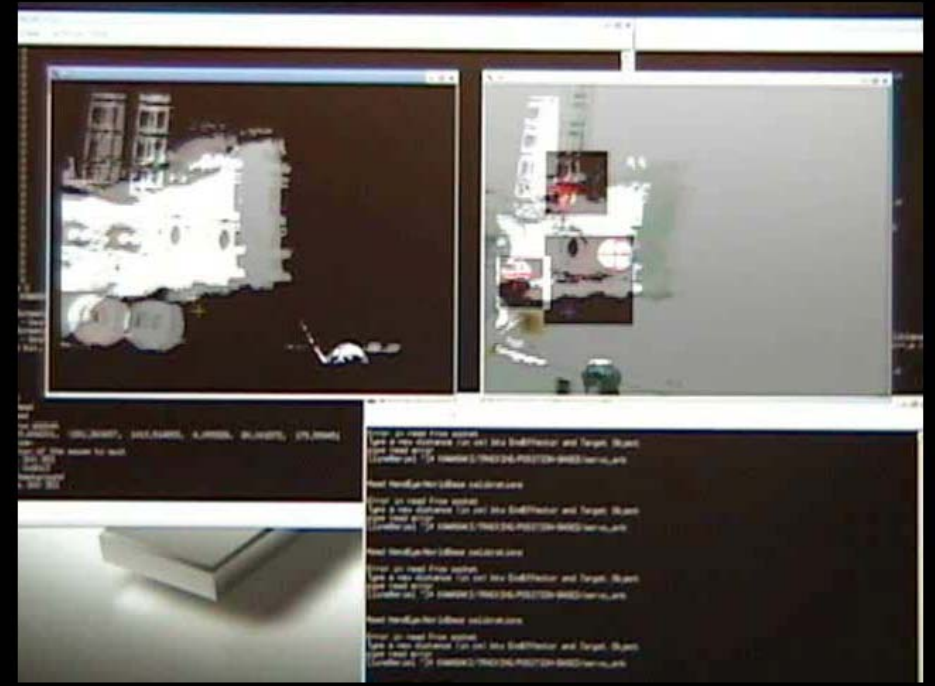

\section{Control Arbitrator}

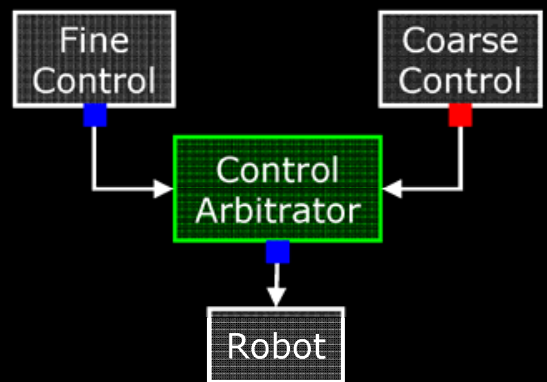

$\square$ Determines which module input to use to control the robot 
Line Tracking System (video)

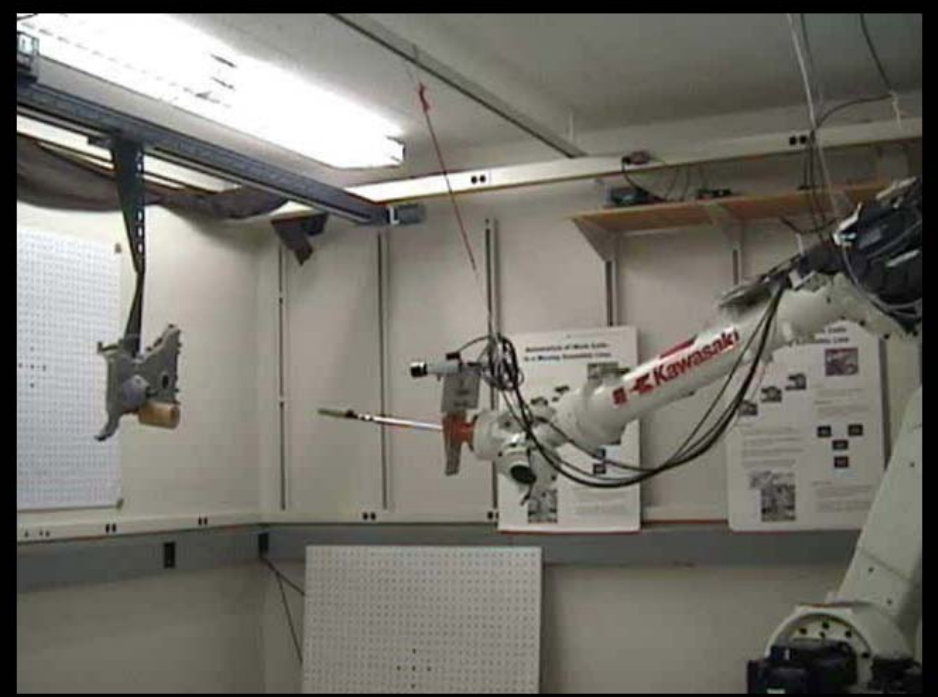

Distributed and Hierarchical

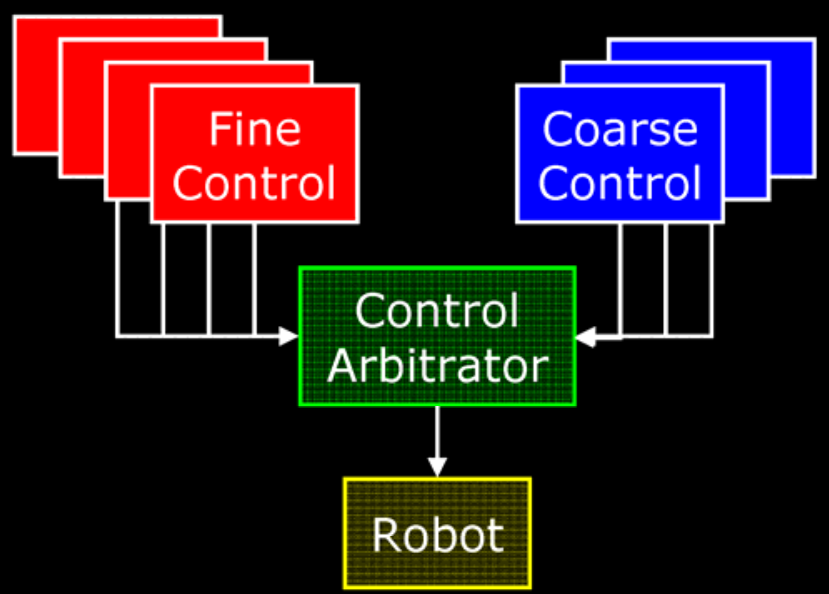




\section{Fine Control Alternative \#1:}

Model-based Tracking
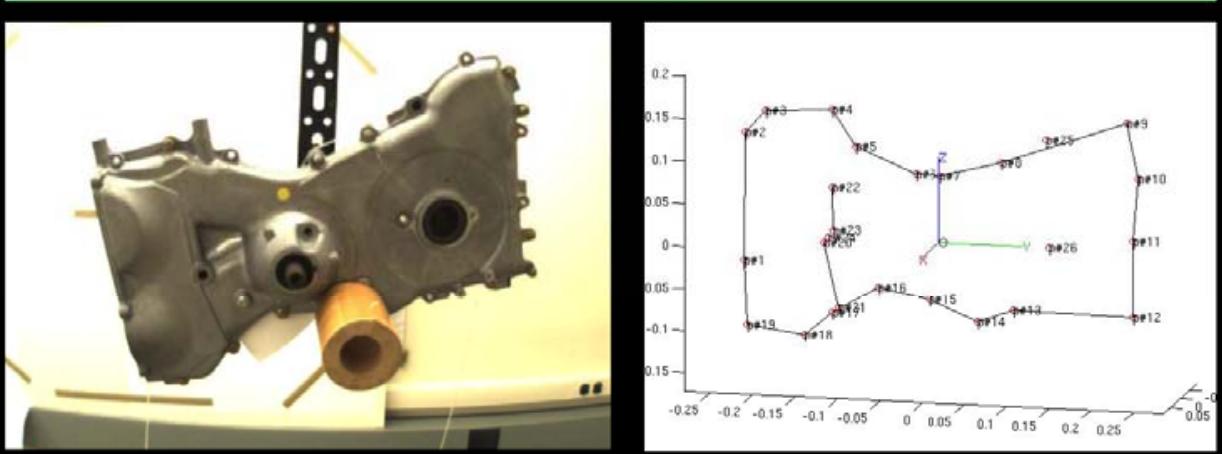

$\square$ A model of the "salient" features of the object is constructed (e.g., straight lines).

$\square$ Model is projected to the image, and by matching the model features in the scene, the pose of the ${ }_{15}$ object is calculated.

tikstop stice 9104

\section{PURDUE}

Model-based Tracking (video)

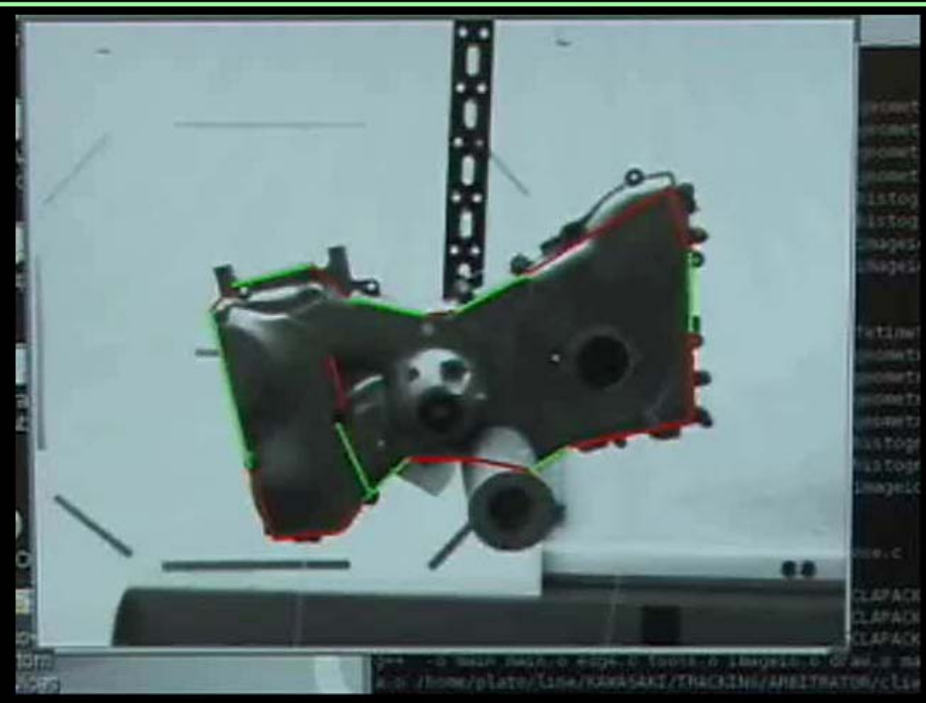

Actual processing speed 


\section{PURDUE}

Model-based Tracking (video)

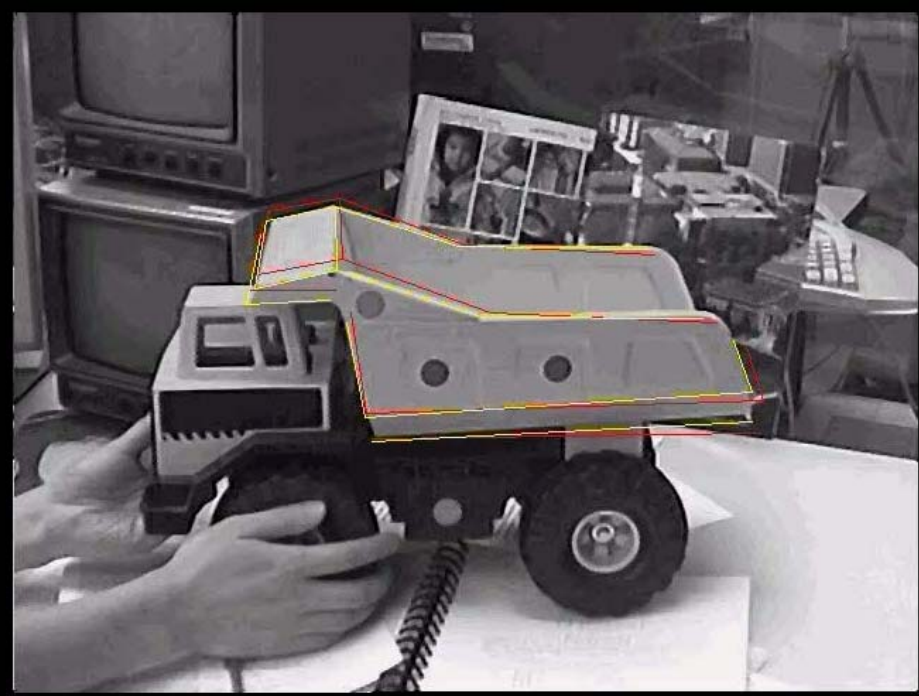

Model-based Tracking (video)

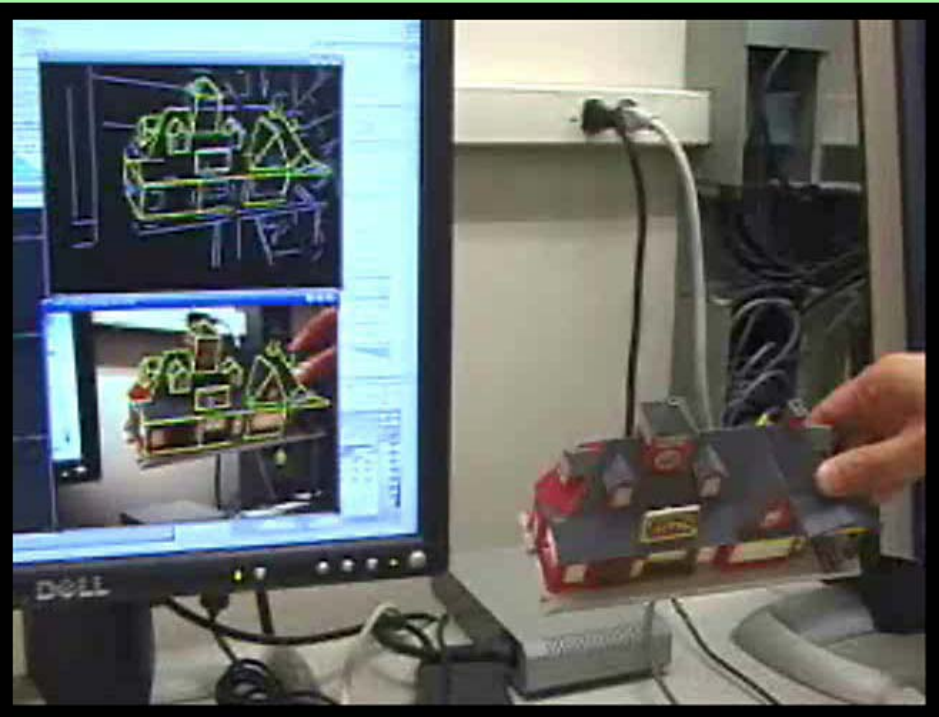




\section{PURDUE}

Multi-loop Fine Control System

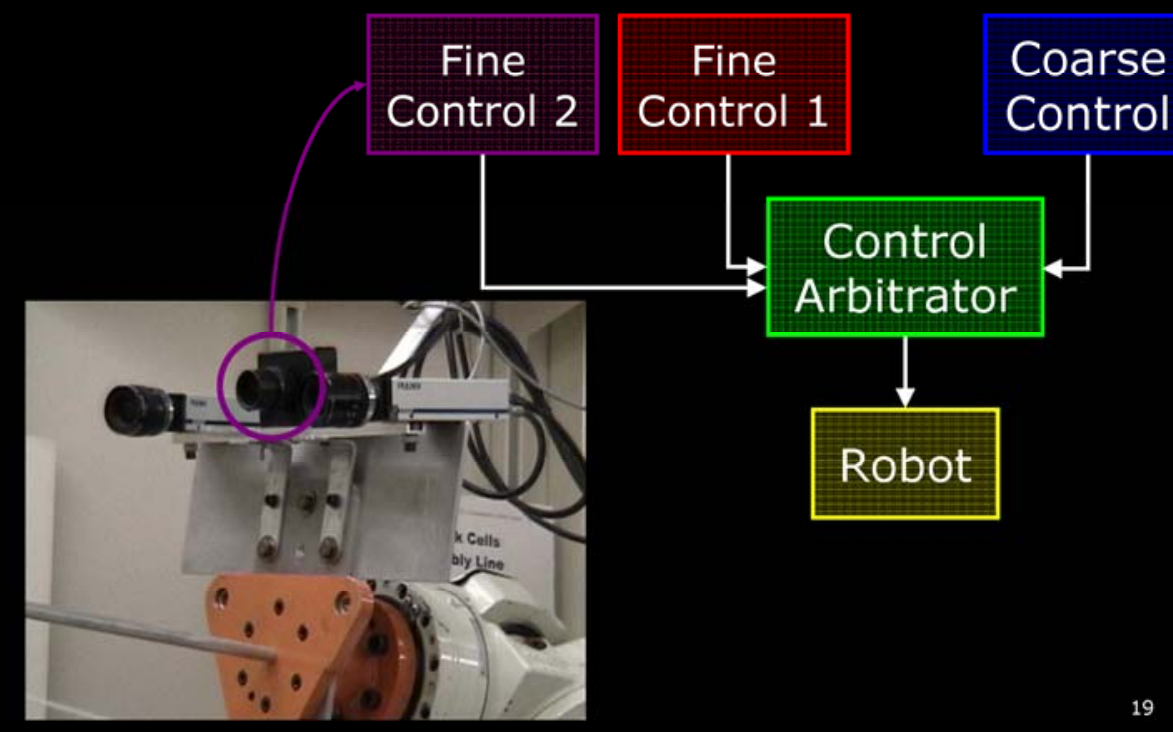

Workshop slide \#108

Single-loop Fine Control System

PURDUE

Under Occlusion (video)

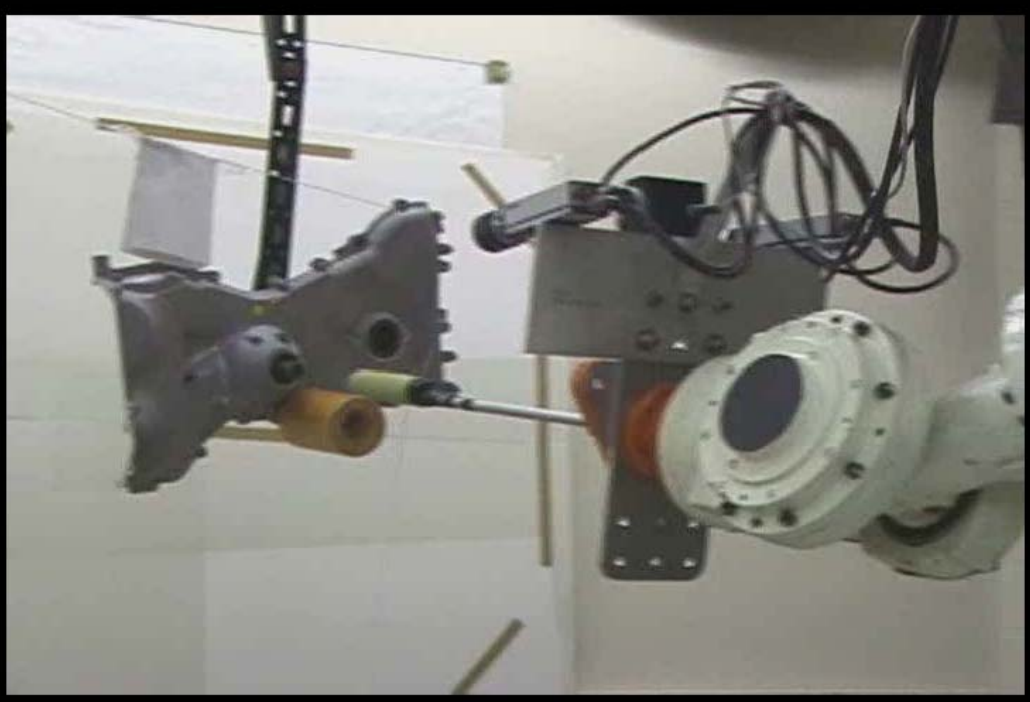


Multi-loop Fine Control System PURDUE Under Occlusion (video)

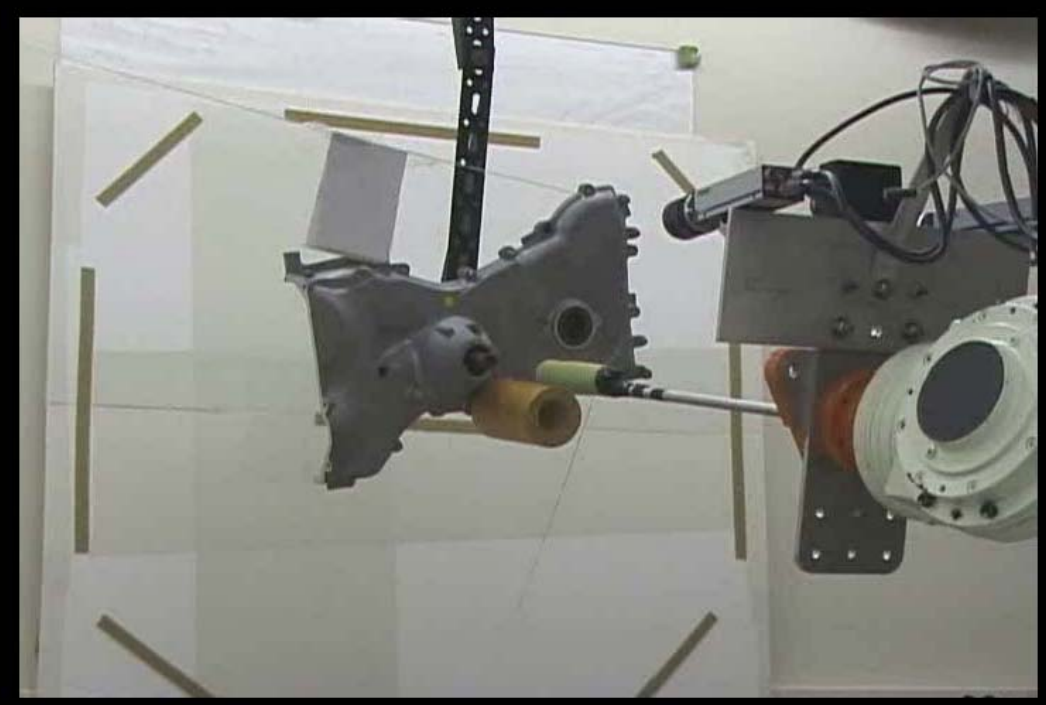

Multi-loop Fine Control System

PURDUE

Under Occlusion (video)

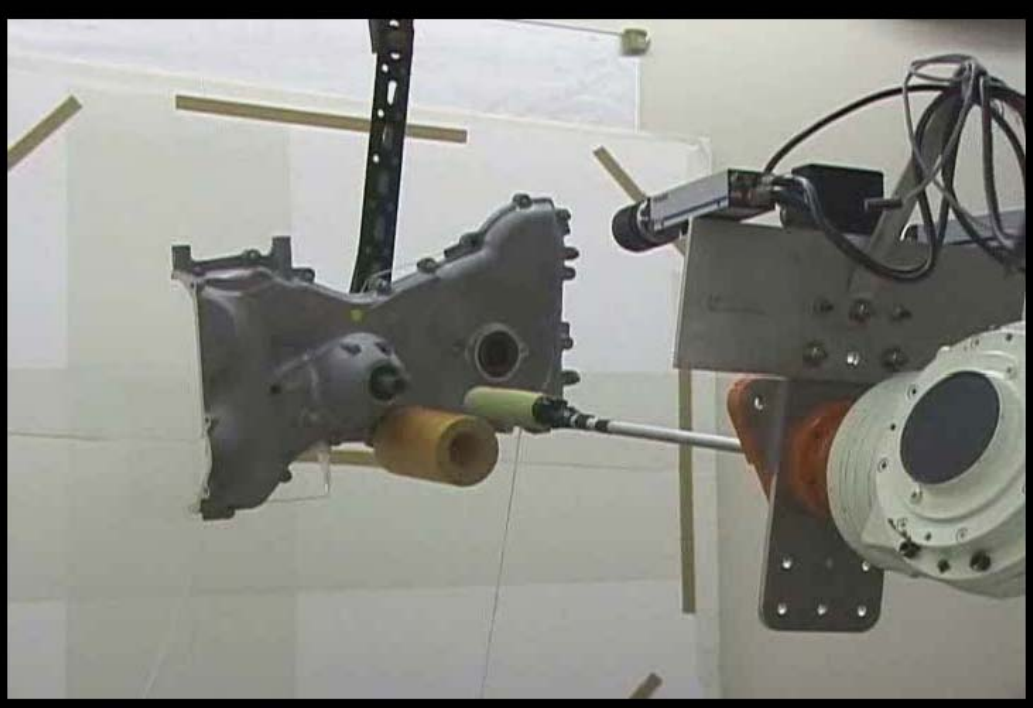


Single-loop Fine Control System PURDUE Under Illumination Change (video)

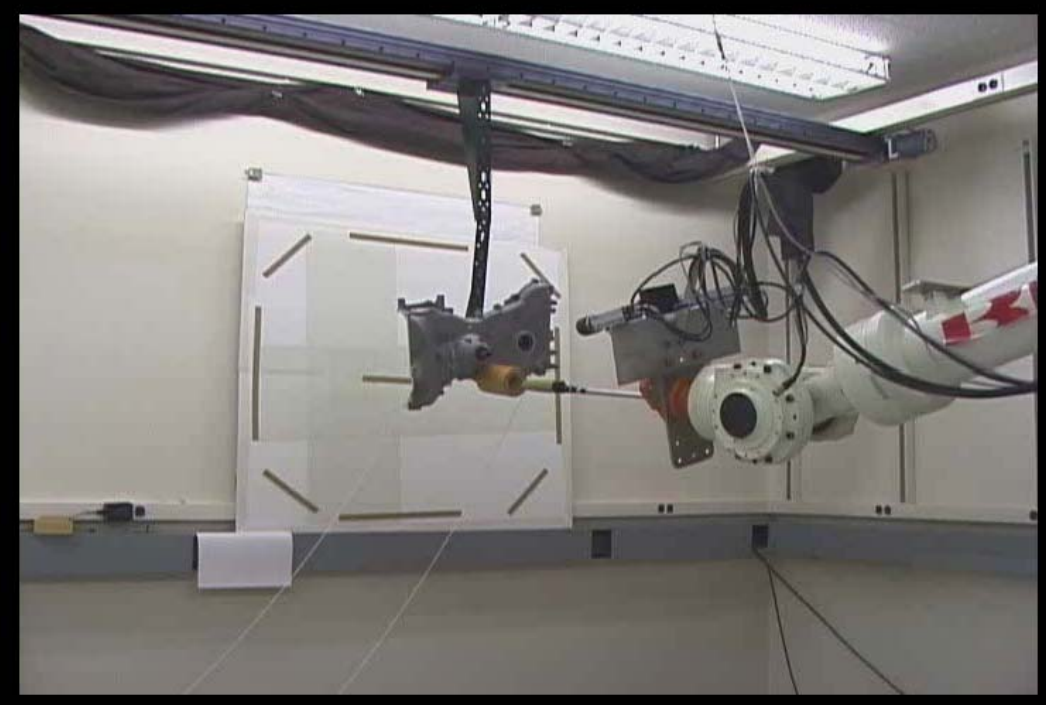

Multi-loop Fine Control System

PURDUE

Under Illumination Change (video)

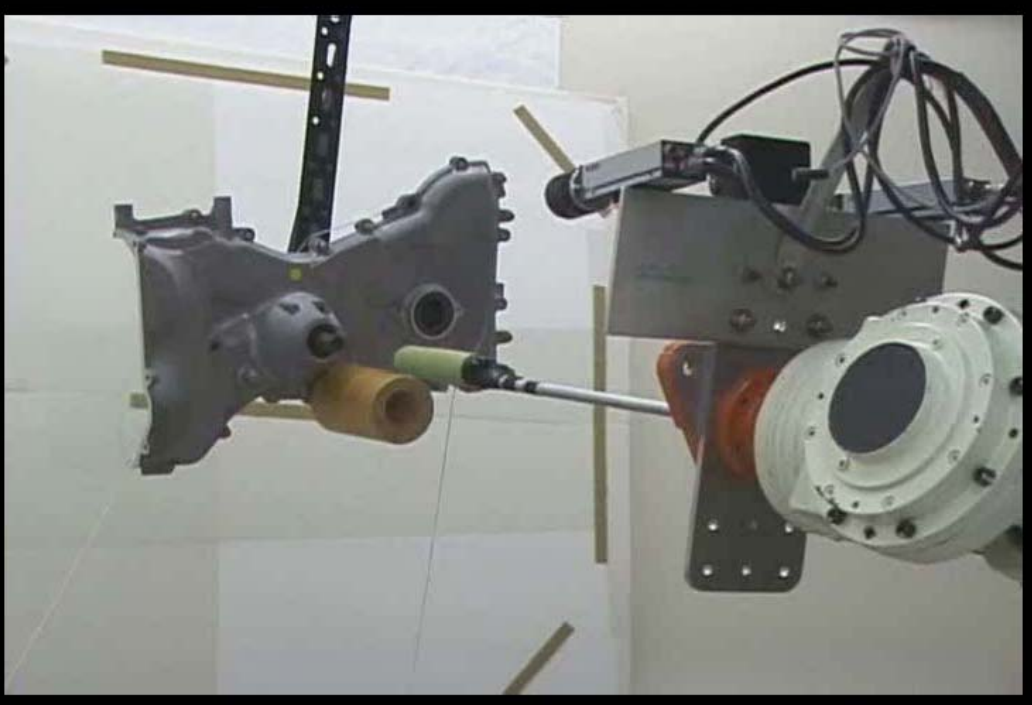




\section{For More Information}

$\square$ Please visit our website:

http://rvl .ecn. purdue.edu 


\section{Visual SLAM: Past, Present \& Future

\author{
Motilal Agrawal, Kurt Konolige, Joan Sola \\ SRI International \\ NIST Workshop on \\ Dynamic Measurement and Control for \\ Automated Manufacturing \\ October 10-11,2007
}

www.ai.sri.com/ agrawal, konolige

workshop slide \#1 15

Overview of the talk

$10 / 09 / 07$

Introduction to Visual Odometry \& SLAM

History

Visual Odometry Principle

Current Status and Directions

Results on various datasets 


\section{Visual Odometry \& SLAM}

Task

- VO: estimate the pose of a vehicle

- SLAM: Build maps and stay localized in this map

Sensors

- Accelerometers/IMU accumulate error rapidly

- Wheel Odometry is subject to slip, sliding

- GPS (WAAS) is accurate to 3-5 $\mathrm{m}$ in the best case in open outdoor terrain; is worse under tree canopy, inside buildings and is subject to jamming;

- Visual Odometry (VO) has tremendous potential

- Can complement other sensors

\section{Applications}

- Estimating 6 DOF pose of objects on the assembly line

- Estimating the pose of a robot indoors

- Autonomous navigation

workshop slide \#117

\section{History}

\section{Cameras are cheap now}

- Stereo cameras work best for visual SLAM

Computing power has gone up

- Specialized hardware for stereo exist

Vision algorithms for structure from motion are now viable

- Maturity in Computer Vision

A few systems around the globe for real time visual SLAM

- SRI has been developing Visual Odometry for three years now 
VO principle

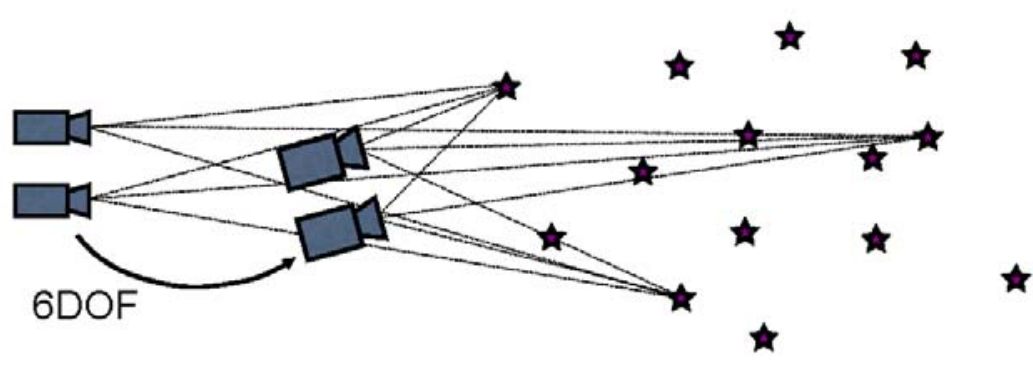

Images with 3D information from stereo

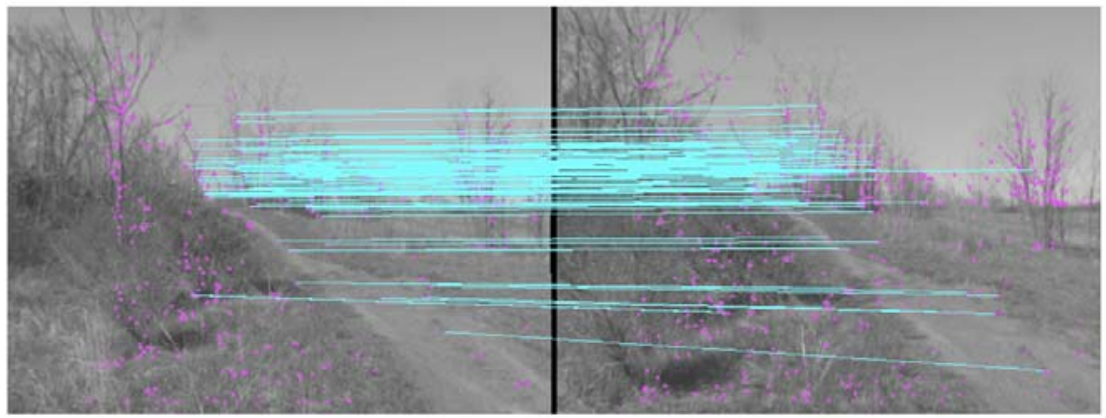

workshop slide "119

\section{VO provides 6DOF relative motions}

Use of Key frames and Window mesh reduces drift

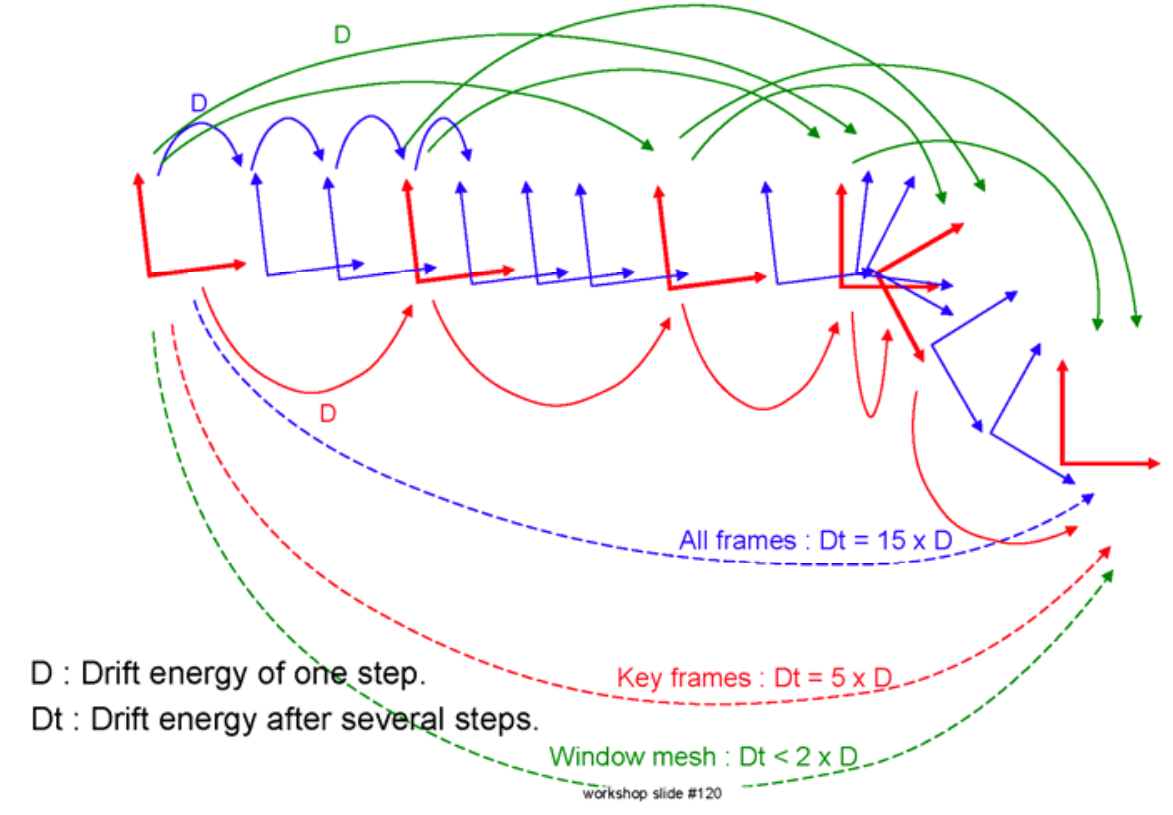




\section{Indoor Feature Tracking}

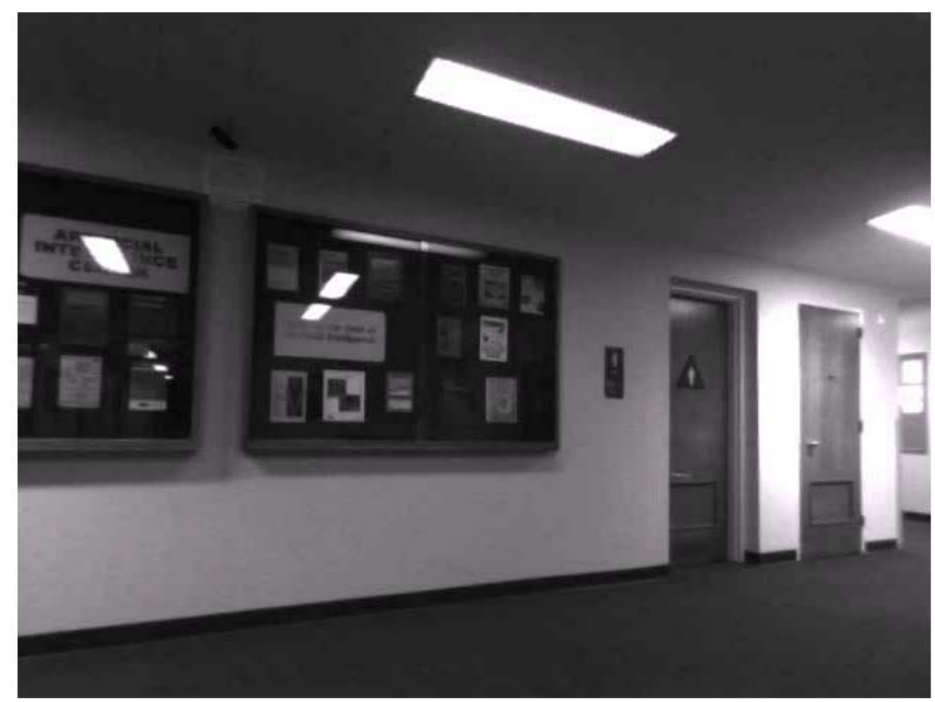

\section{Indoor office environment}

VO in an indoor office corridor

Loop of 40 meters

Loop closure error $<0.4 \mathrm{~m}$

Crosses show the trajectory forward trajectory in blue return trajectory in red The 3D tracked features are marked as dots

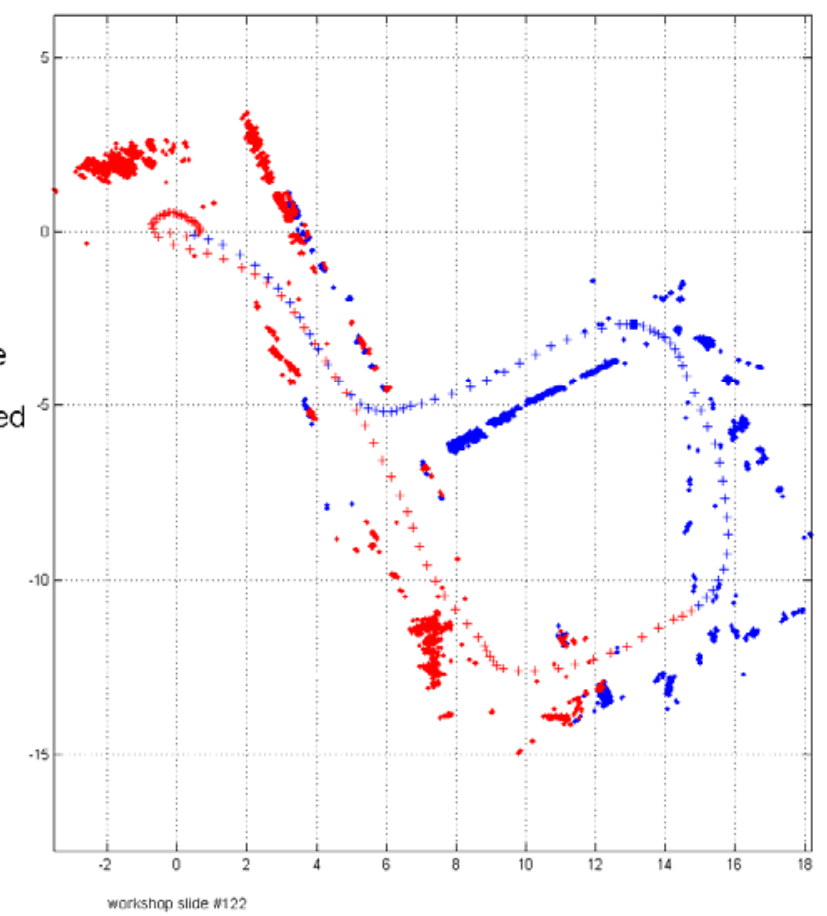




\section{Current status}

$>$ Practical, inexpensive, real-time vision based system for localization

Localizes within 1\% error over large distances

- Experimental validation over $9 \mathrm{~km}$ on outdoor terrain

System ideal for autonomous navigation of a robot

Long term drifts minimized through integration with a low cost absolute sensor

- Gravity normal from IMU

$>$ System tested out on datasets from other people

\section{Ongoing work}

Good features for tracking

- Indoor vs outdoor

SLAM and loop closure

- Maps remain consistent in spite of drift

Visual landmarks recognition

- Relocalization

Integration with IMU at the sensor level

- IMU provides an absolute gravity normal to provide the angle corrections

Visual SLAM workshop

- IROS 2007, Nov 2, 2007

- CVPR 2008, June 232008 
Results on datasets provided by other people

10/09/07

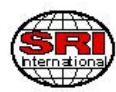

Visual Odometry Example: Urban environment

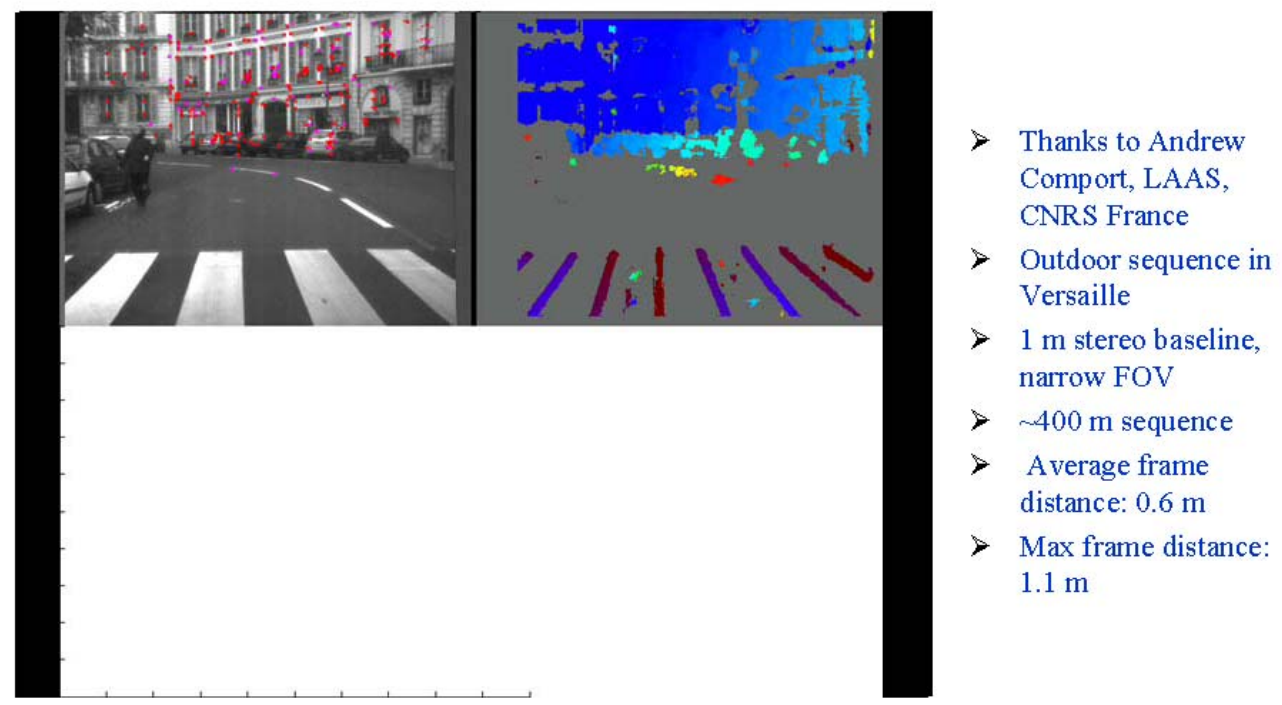




\section{Visual Odometry Example}
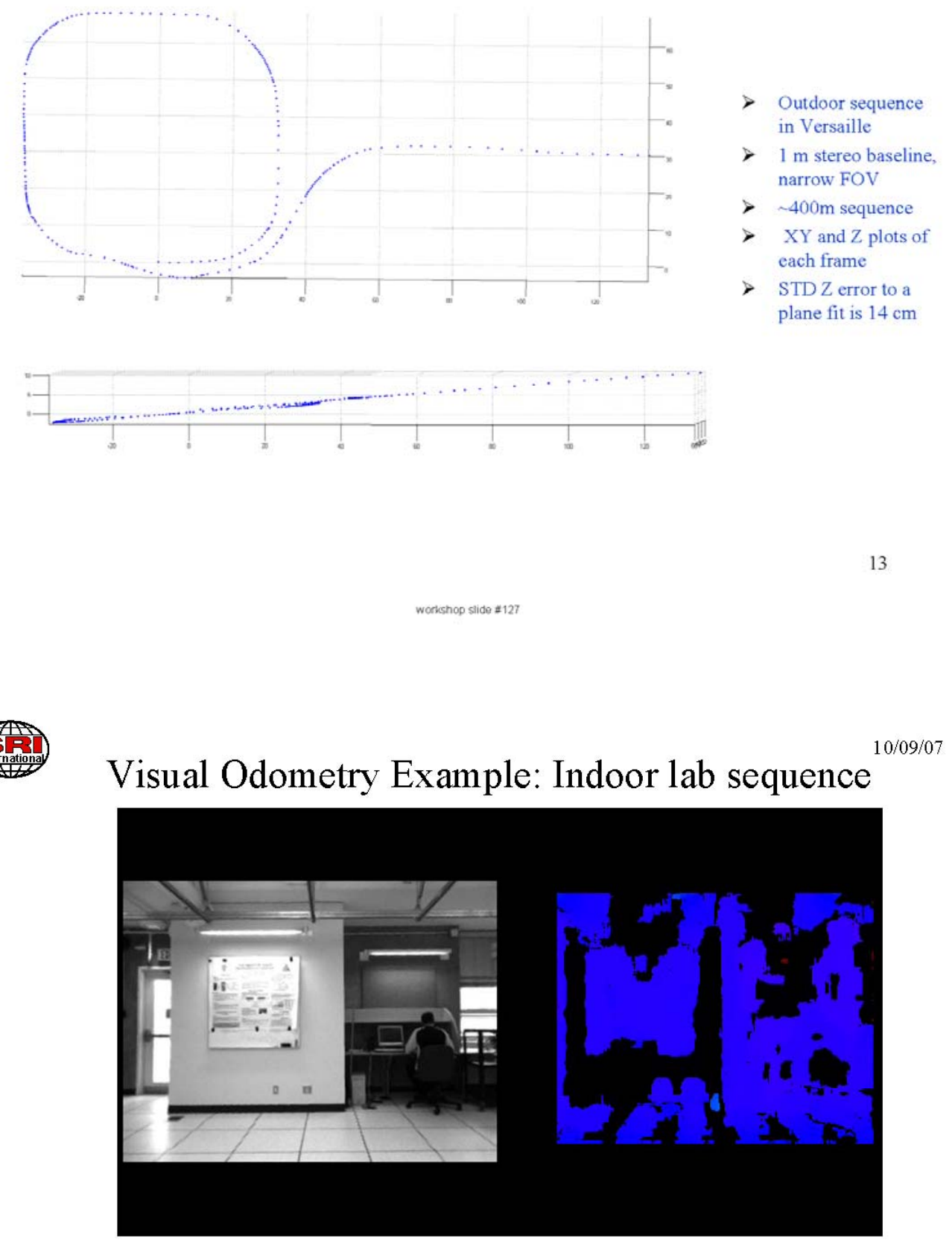

- Thanks to Robert Sim, UBC, Canada

- Indoor lab sequence

- $12 \mathrm{~cm}$ stereo baseline, wide FOV

- $\sim 100 \mathrm{~m}$ sequence, $\sim 1200$ key frames

- 17 tack points in the VSLAM graph 


\section{Visual Odometry Example}

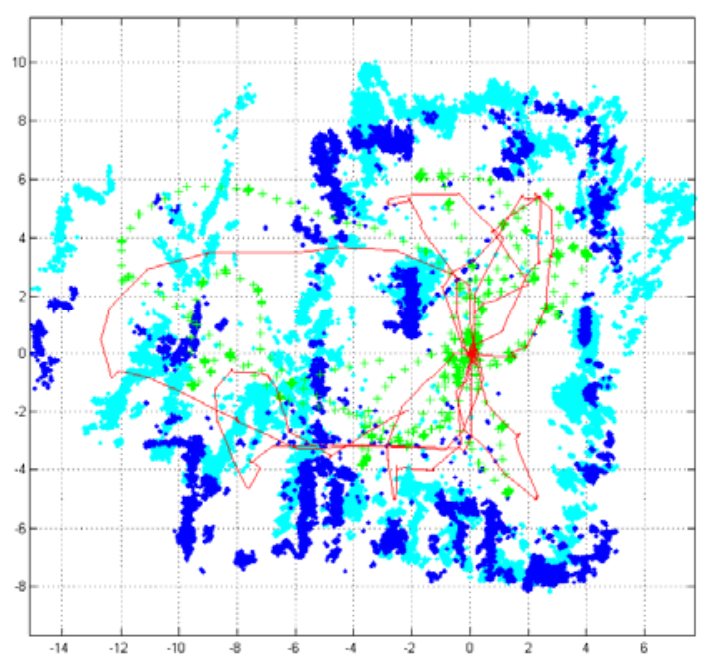

Indoor lab sequence

$>12 \mathrm{~cm}$ stereo baseline, wide FOV

$>\sim 100 \mathrm{~m}$ sequence, $\sim 1200$ key frames

$>$ Green crosses are uncorrected VO; cyan environment points

$>$ Red segments are VSLAM-corrected poses; blue environment points

$>17$ tack points in the VSLAM graph

\section{Loop closure error}

Robot driven in a loop over waypoints accurately survey ed using RTK GPS

$>$ Loop closure error $2.8 \mathrm{~m}(<1 \%)$

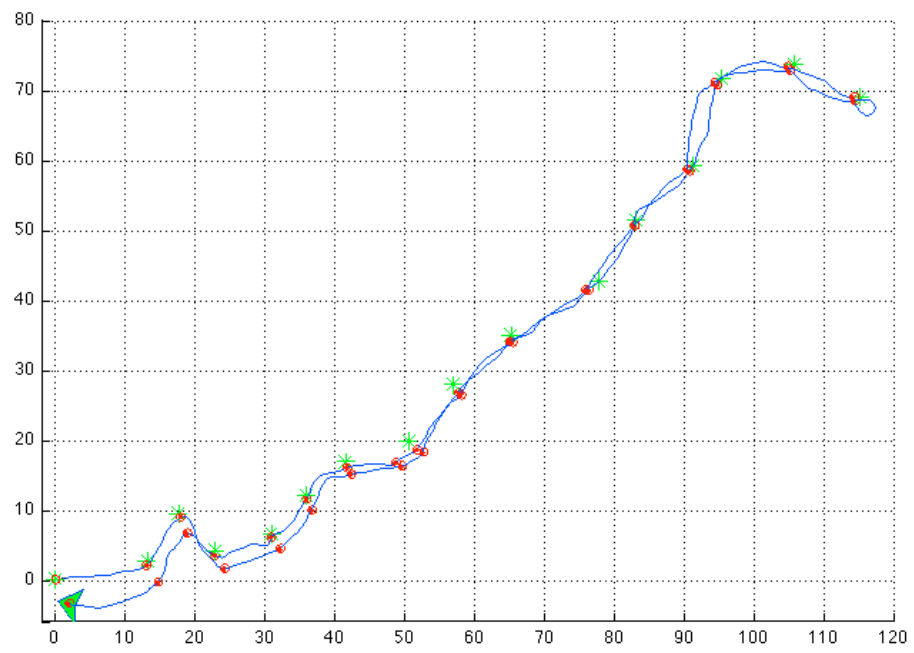

workshop side \#130 
a very large outdoor sequence (t) RTK GPS (ground truth) path length

$50 \mathrm{~m}$ end error $(0.5 \%)$

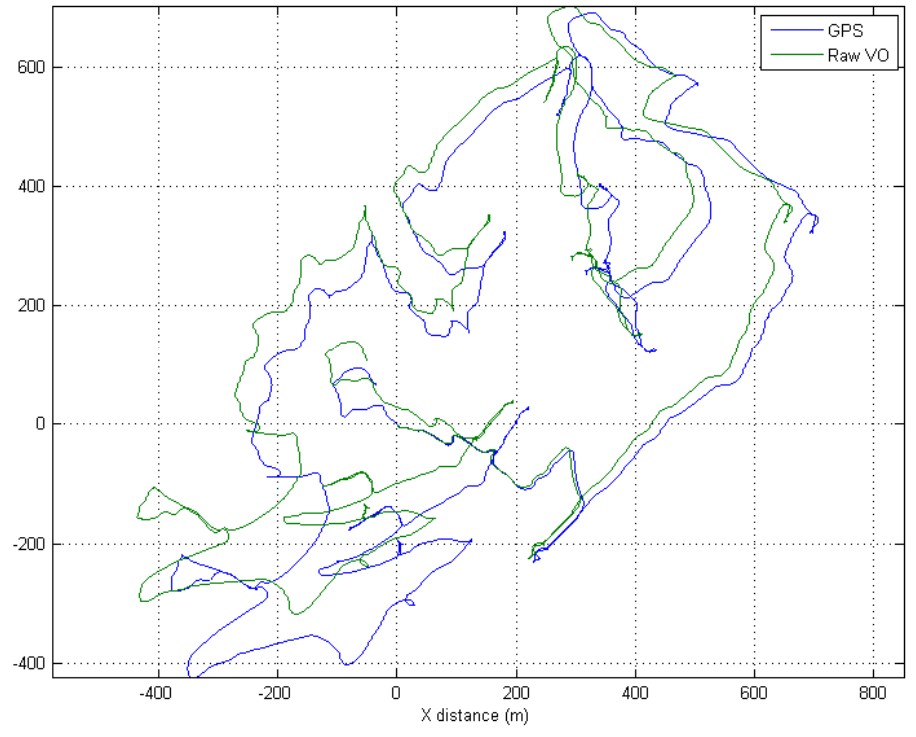

workshop slide\#131

\section{Exrsion with IMU using Kalman Filter} ind error $(0.1 \%)$

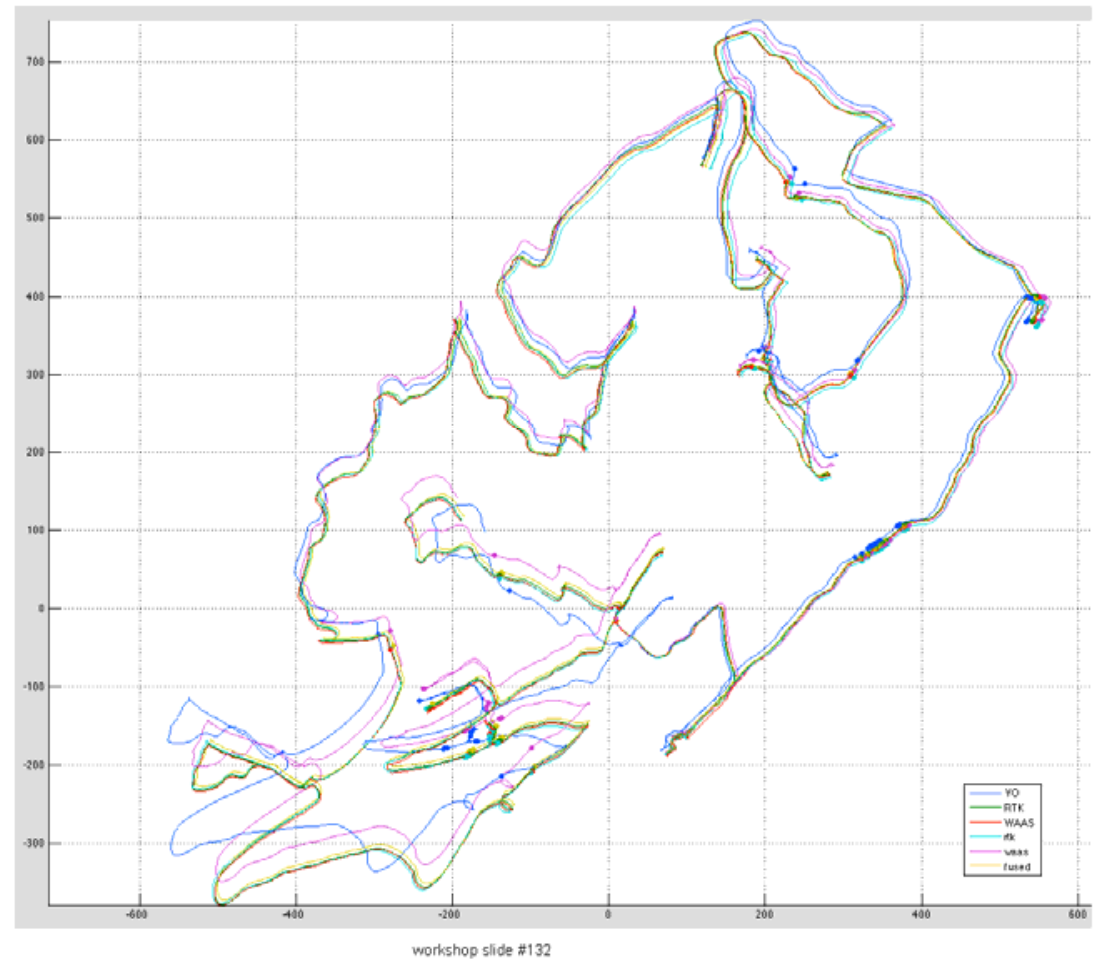




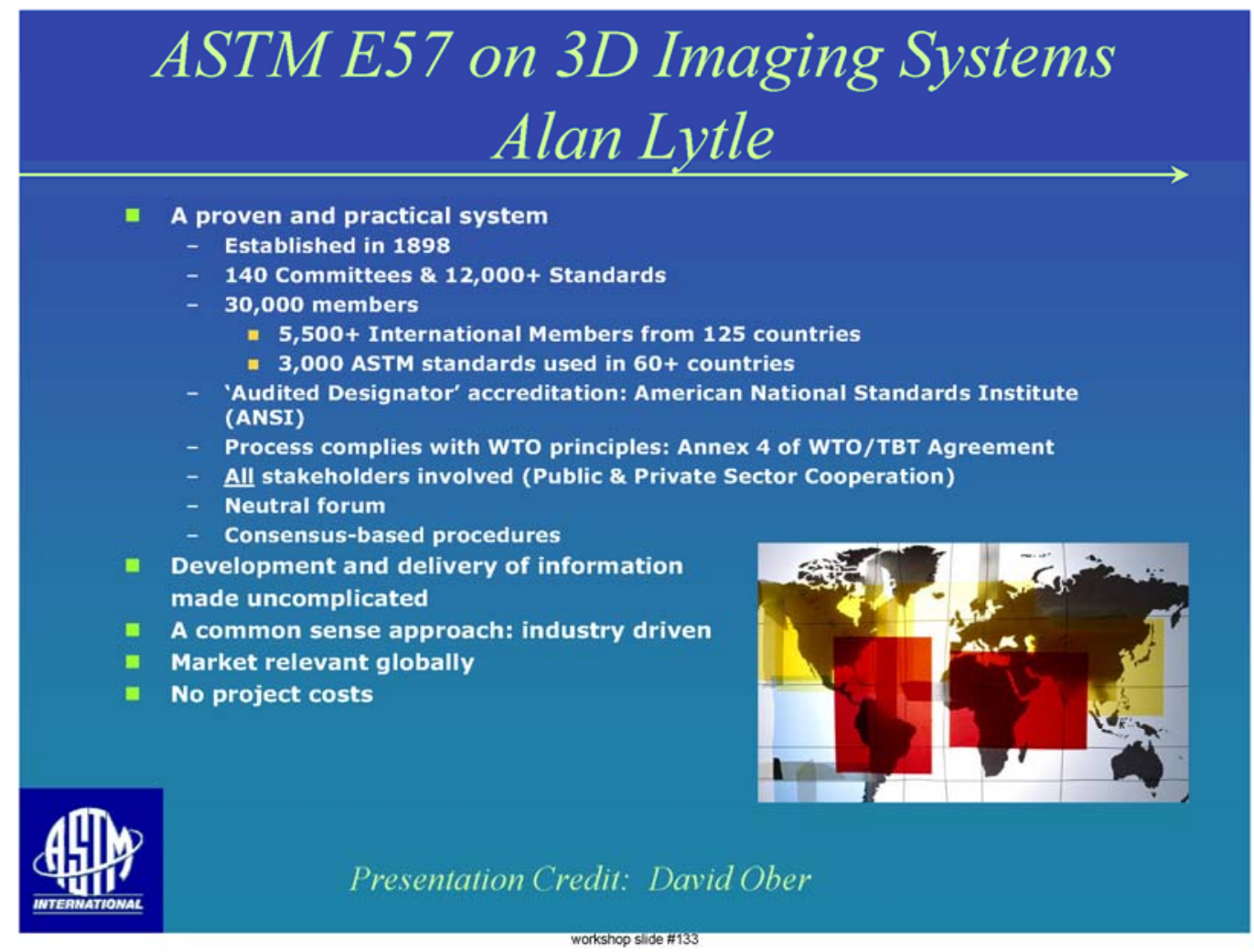

workshop silde \#133

\section{E57 on 3D Imaging Systems}

-Organized June 7, 2006 by Industry

- Two Organized Meetings since E57 creation. (This does not include individual subcommittee meetings, task groups, or work items)

口Current E57 Roster: 113 Individuals and Organizations 


\section{E57 Committee Officers}

- Chairman: Alan Lytle, NIST

- Vice-Chairman: Alan Aindow, Leica Hds

- Recording Secretary: Steve Hand, Survice

- Membership Secretary: Tom Greaves, Spar Point Research

- Member at Large: Tad Fry, AnheuserBusch Incorporated

- Staff Manager: Pat Picariello, ASTM

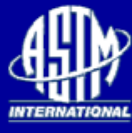

orkshop silice \$135

\section{E57 Scope}

The development of standards for 3D imaging systems, which include, but are not limited to laser scanners (also known as LADAR or laser radars) and optical range cameras (also known as flash LADAR or 3D range camera).

The initial focus will be on standards for 3D imaging systems specification and performance evaluation for applications including, but not limited to:

Construction and Maintenance

Surveying

Mapping and Terrain Characterization

Manufacturing (e.g., aerospace, shipbuilding, etc.)

Transportation

Mining

. Mobility

. Historic preservation

. Forensics

The work of this Committee will be coordinated with other ASTM Committees and outside organizations mutual interest.

workshop slice \$136 


\section{E57 Subcommittees}

口E57.01: Terminology

口E57.02: Test Methods

口E57.03: Best Practices

E57.04: Interoperability

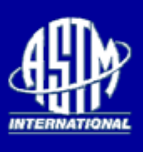

\section{E57.01 Terminology}

-Scope: The Development of terminology commonly used for 3D imaging systems. The work of this subcommittee will be conducted with other ASTM E57 Subcommittees.

nChairman: Gerry Cheok, NIST

Vice Chairman: Kam Saidi, NIST 


\section{E57.01 Terminology - Update}

- January 2007 - Approved ASTM E2544, includes

- 8 terms specific to 3D imaging systems

- Other commonly used metrology terms as defined by other standards

- Accuracy

- Bias

Precision

- Calibration

- Compensation

- Conventional true value

- Error of measurement

- Indicating (measuring) instrument

- Limiting conditions

- Maximum permissible error

- Measurand

Random error

Rated conditions

Relative error

Repeatability

Reproducibility

Systematic error

True value

Uncertainty of measurement

- May 2007 - Second ballot for additional terms to ASTM 2544

- 15 terms submitted

- 8 terms approved

4닌

- Resolved most of negative votes at June 2007 meeting

\section{E57.01 Terminology-Approved Terms}

3D imaging systems

Angular increment

Beam propagation ratio

Beam width

First return

Flash LADAR

Instrument origin

Last return

Workshop silco \$139 


\section{E57.01 Terminology}

Negatives to be Resolved and Re-balloted

\section{D imaging systems}

- Beam diameter

- Beam divergence angles

- Control points

- Registration

- Stigmatic beam

- Spot size

\section{4पा

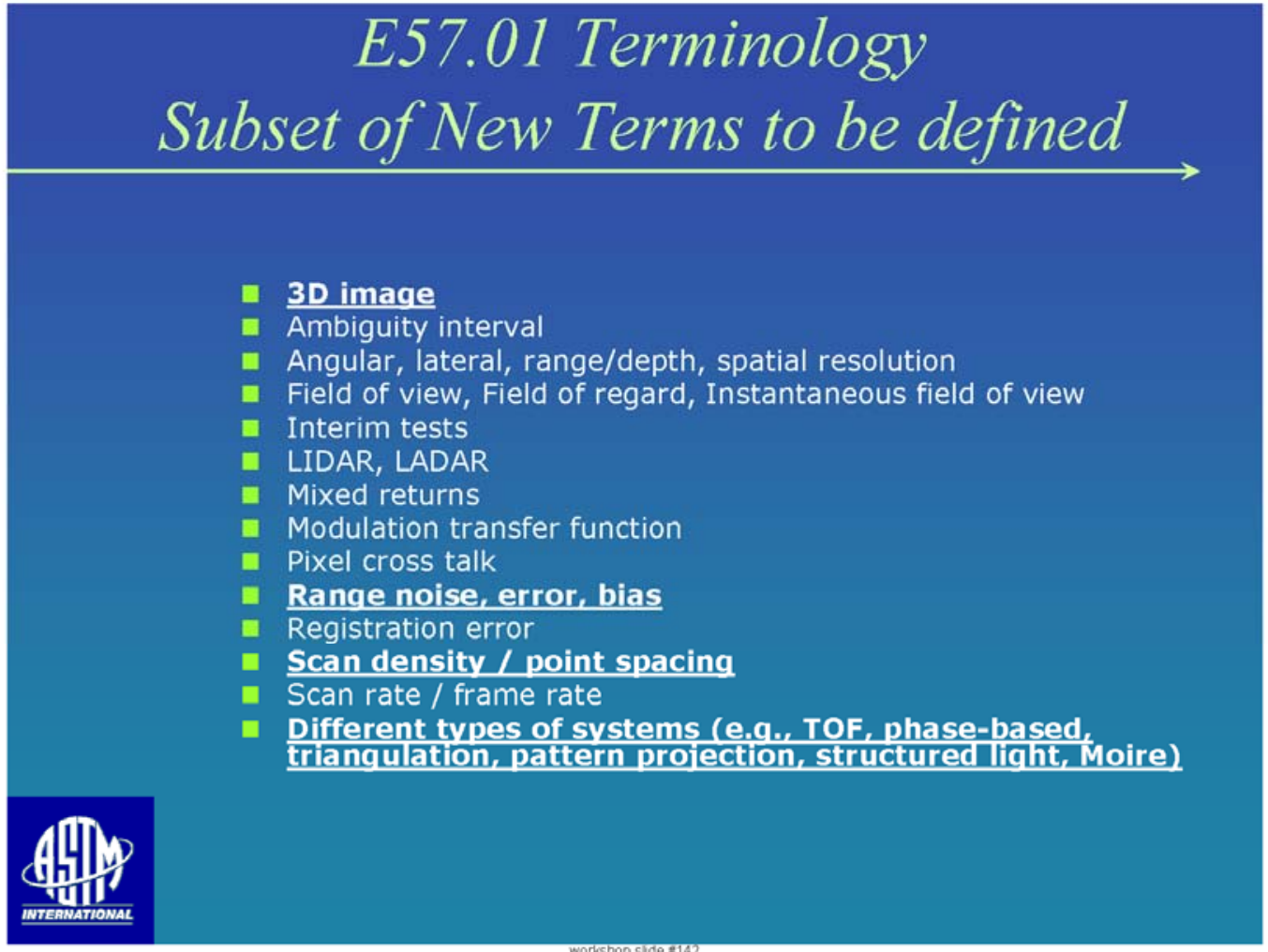

Workshop şत्र श142 


\section{E57.01 - Future Tasks}

continue work on approximately 40 new terms

-Teleconferences every 2 weeks

contact: Gerry Cheok, NIST cheok@nist.gov

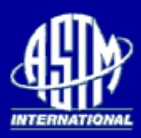

\section{E57.02 Test Methods}

$\square$ Scope: The development of standard protocols that will be used to characterize 3D Imaging System performance.

- Chairman: David Ober, Metris

vice Chairman: Darin Ingimarson, QUANTAPOINT

- Secretary: Mike Garvey, M7 Technologies 


\section{E57.02 Test Methods - Overview}

Each Test Method: Define purpose of test

- Data Collection

- Requirements: Environment stability, lighting, etc.

- Setup General: Hardware (sphere, plane, reflectance) height, IA, Range, etc.

- Setup Instrument specifics: Point Spacing, Dwell time, Data Rate, Internal filter settings, etc.

- Measurements: Scan data (XYZ or RAE, RGB, Signal integrity: SNR or Intensity, etc.), temperature, pressure, humidity, light, wind, etc.)

- Data Analysis

- Data Filters (Allow post processing manufacture filters vs. raw data)

- Conversion Interoperability (data format)

- Algorithms (Process the data)

- Outliers vs. statistically meaningful data

- Results Report

- Manufacture specifications (and how they integrate with the analysis AND results) Data presentation (MPE, STD, Histogram, Mean, RMS, \% Outliers, $\%$ data missing, \% coverage, etc.)

\section{E57.02 Test Methods - Update}

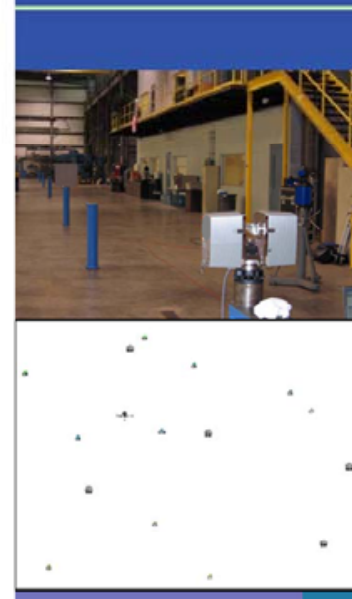

Concentration: Scanners with Maximum Test Range $<120$ meters

Rance Uncertainty Protocol: (Included Data Collection, Analysis, \& Report) Tested at M7 Technologies \& NIST.

Protocol undergoing next revision.

- Angle Uncertainty Protocol: Two data collection approaches tested: Spheres and Flat Planes at M7 Technologies \& Quantapoint respectively.

(Analysis \& report still TBD)

Planar \& Spherical Analysis Simulation: Analysis of existing Plane \& Sphere Fit routines on detecting unbiased instrument Range, Azimuth, \& Elevation uncertainty 

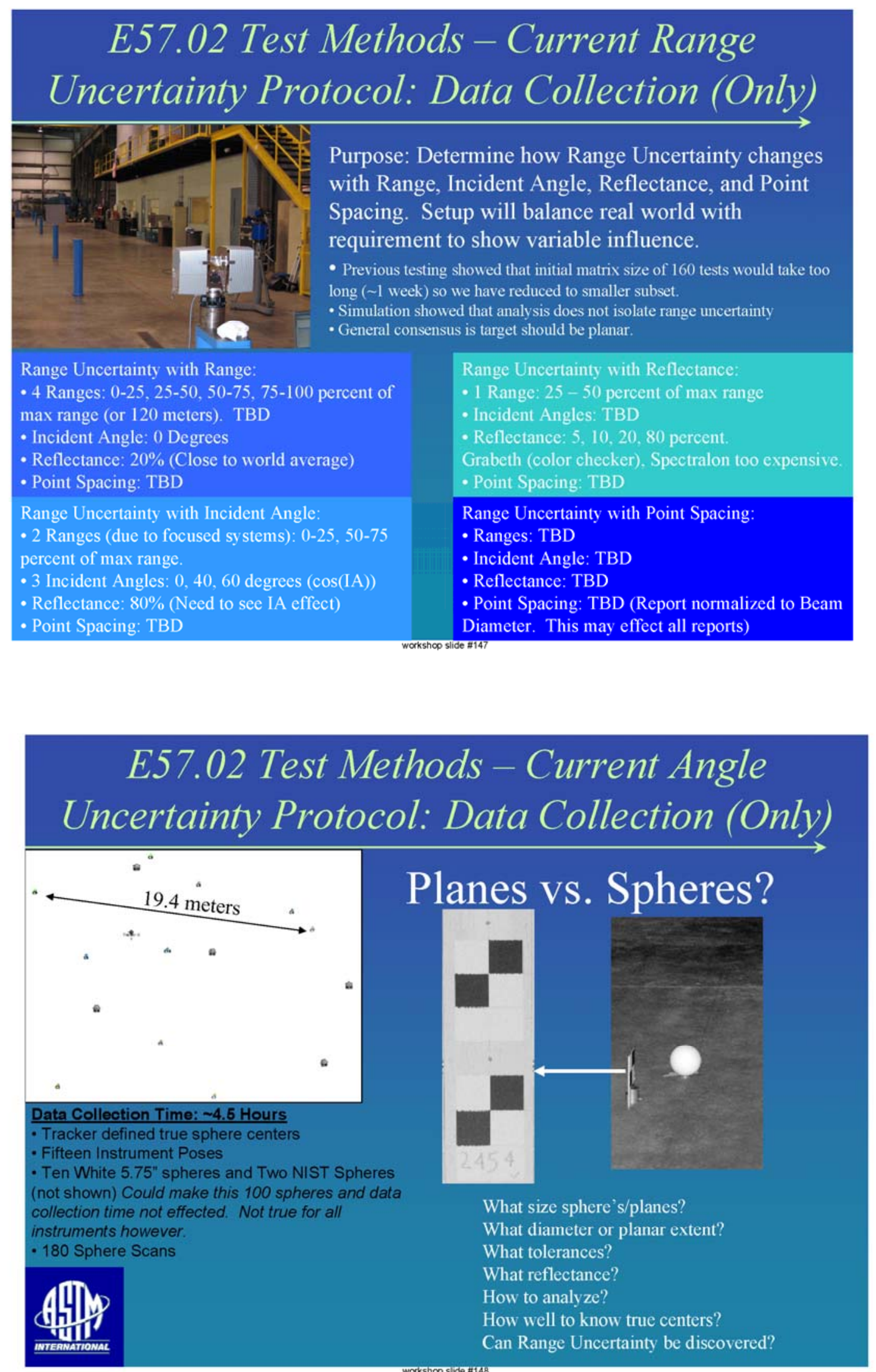

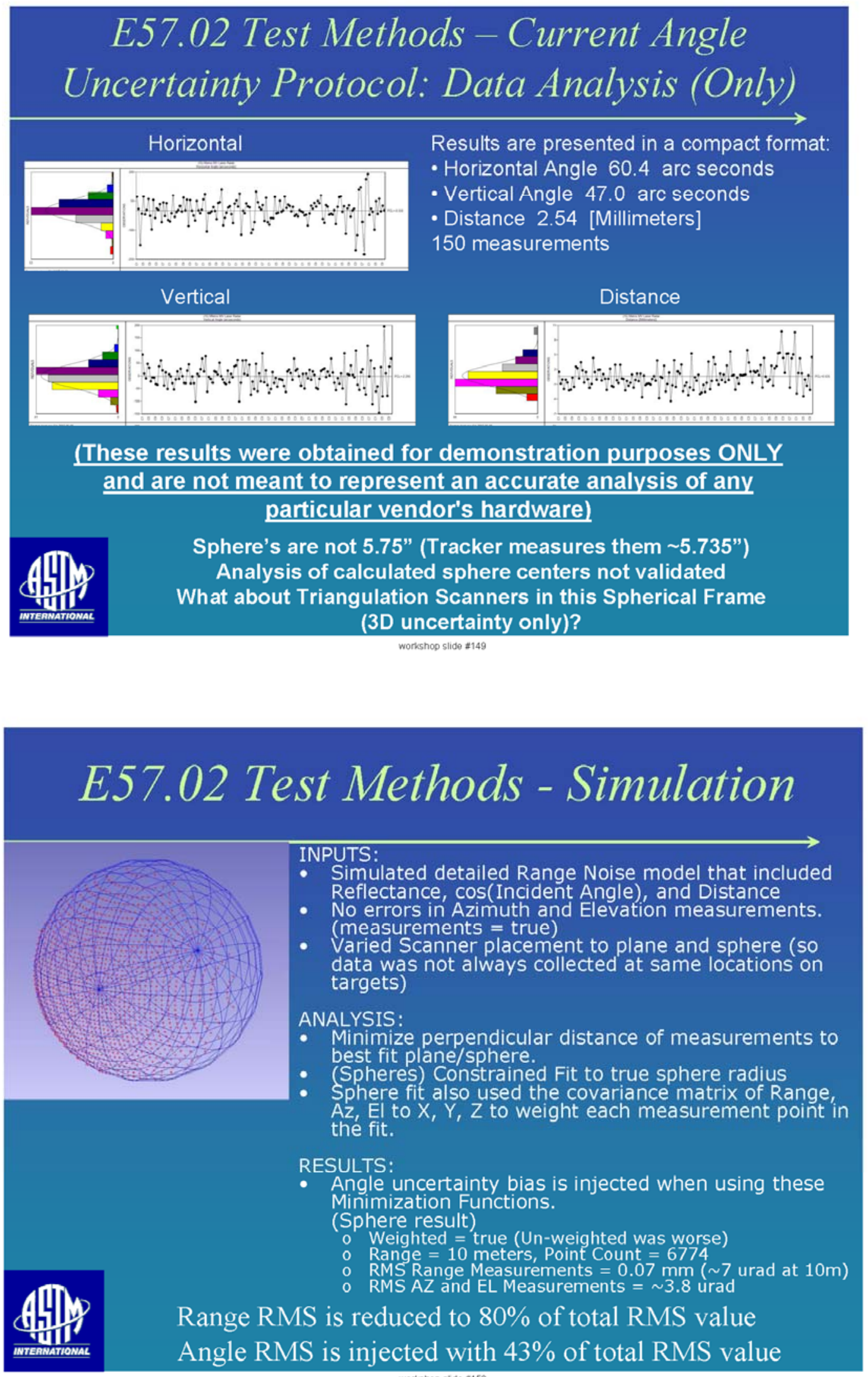

workshop side \#150 


\section{E57.02 Test Methods - Up Next}

Meet Bi-weekly (or monthly as work progresses)

- Examine ISO/TC Terrestrial Laser Scanners Protocol: UNIBWM - 85577

- Complete the Range Uncertainty Data Collection Protocol

- Continue Analysis Simulation Study

- Develop best fit routines to reduce bias transfer between 3 dimensions for planes and spheres. (NIST has an algorithm being developed)

- Committee can decide to live with this bias transfer

- Develop the Range Uncertainty Analysis Protocol

- Develop the Range Uncertainty Results Report

- Test the new Range Uncertainty Protocol

- Leica and Faro have indicated that they are willing to run these tests at their facilities.

- NIST \& M7 Technologies continue to also provide their facilities for testing as well.

- Future Tasks

- Resolution uncertainty

- Dynamic Range

- Adapt tests to other instrument technologies (line scanners, Airborne scanners, etc.)

\section{E57.03 Best Practices}

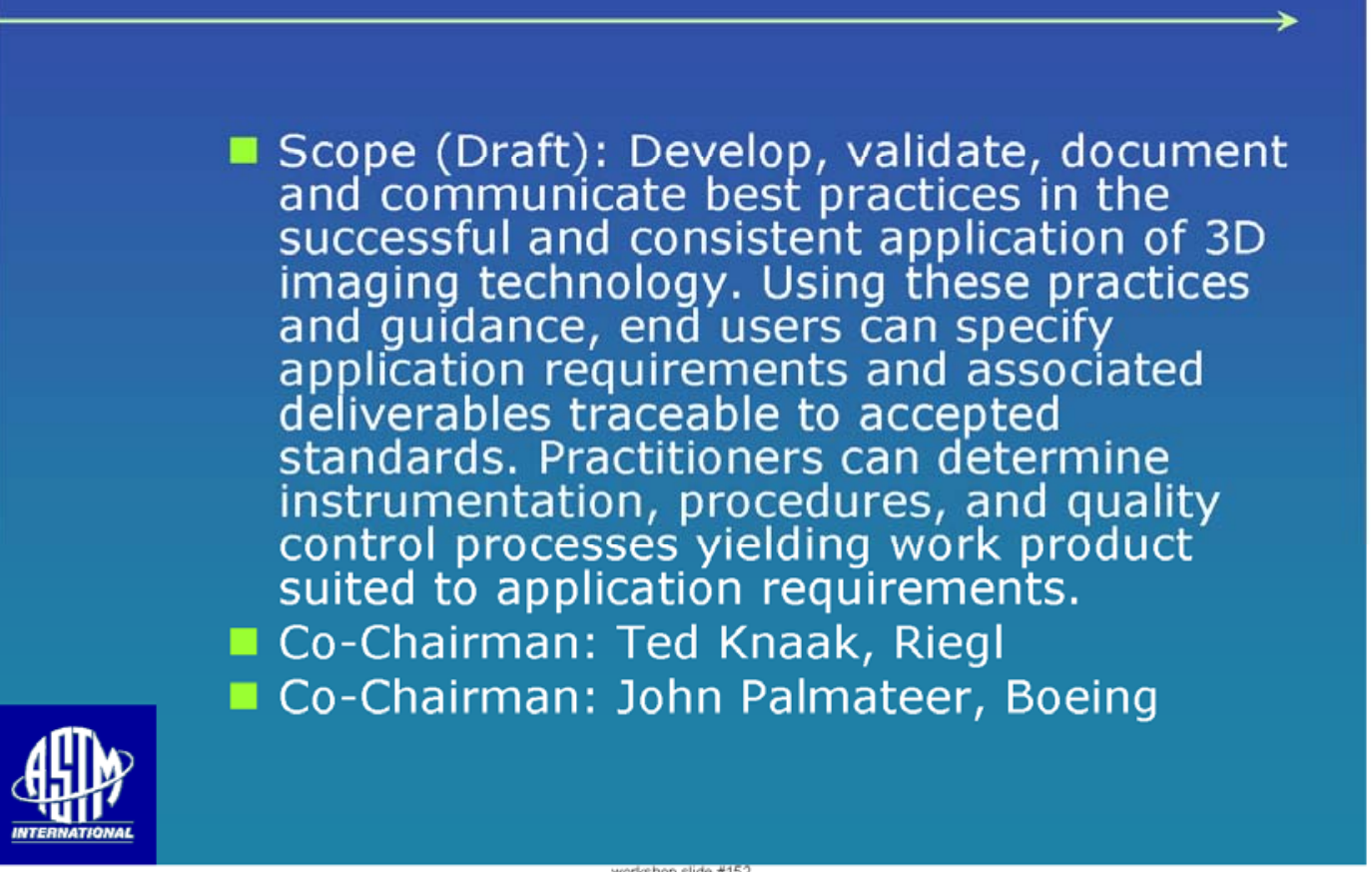

workshop side $\$ 152$ 


\section{E57.03 Best Practices - Structure (July 5, 2007)}

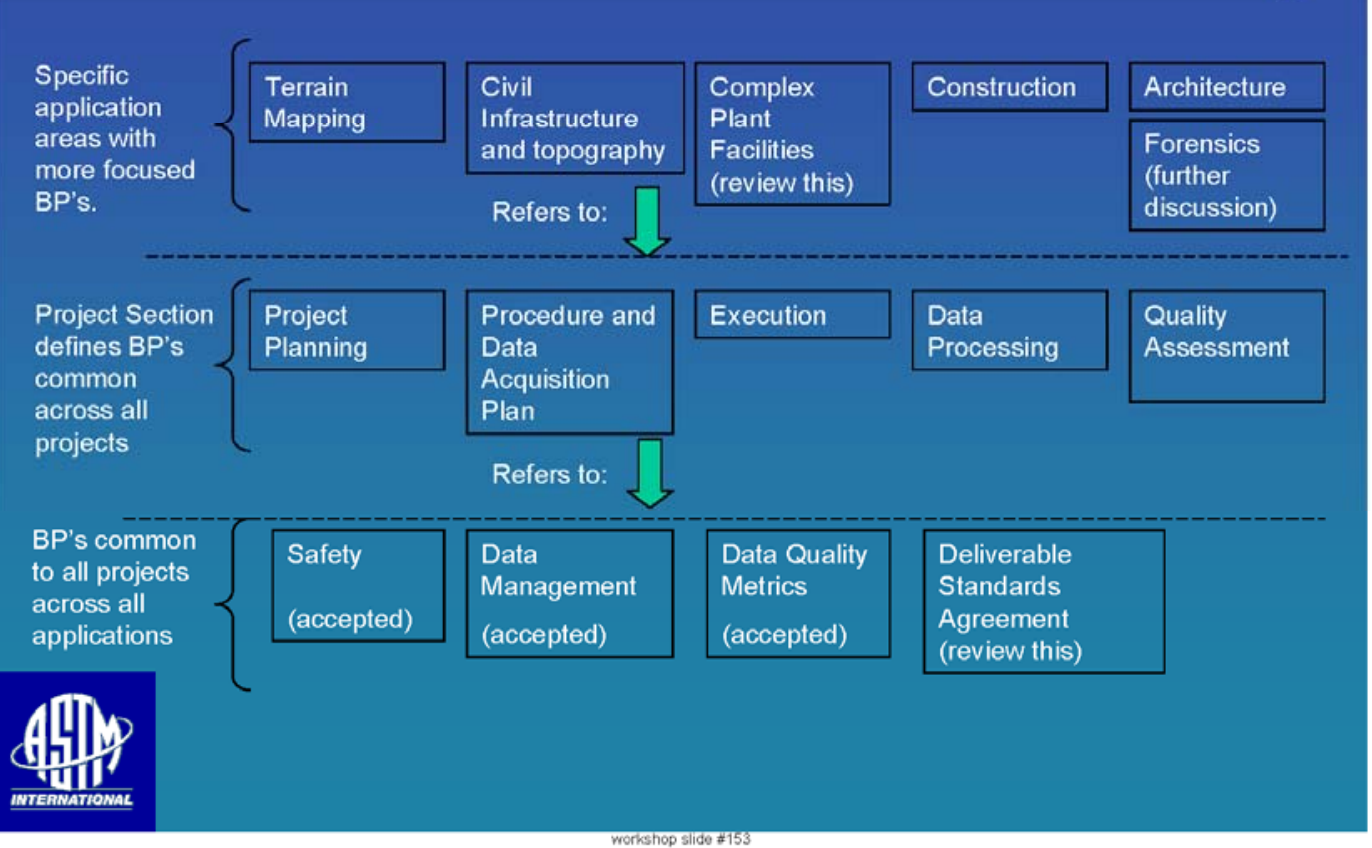

\section{E57.03 Best Practices - Future work}

V Vote on Scope Statement

- Develop Document Management (MicroSoft Teamshare or ASTM tool)

- Develop General Best Practice Outline (Introduction, Scope, Contents, etc.)

Develop Task Groups:

- Data Management Best Practice

- Safety Best Practice

- Data Quality Best Practice 


\section{E57.04 Interoperability}

- Scope: To develop and promulgate open, standard data exchange mechanisms for 3D imaging system derived data in order to promote its widest possible use.

-Chairman: Gene Roe, Autodesk

vice Chairman: Mark Klusza, Trimble

\section{(니

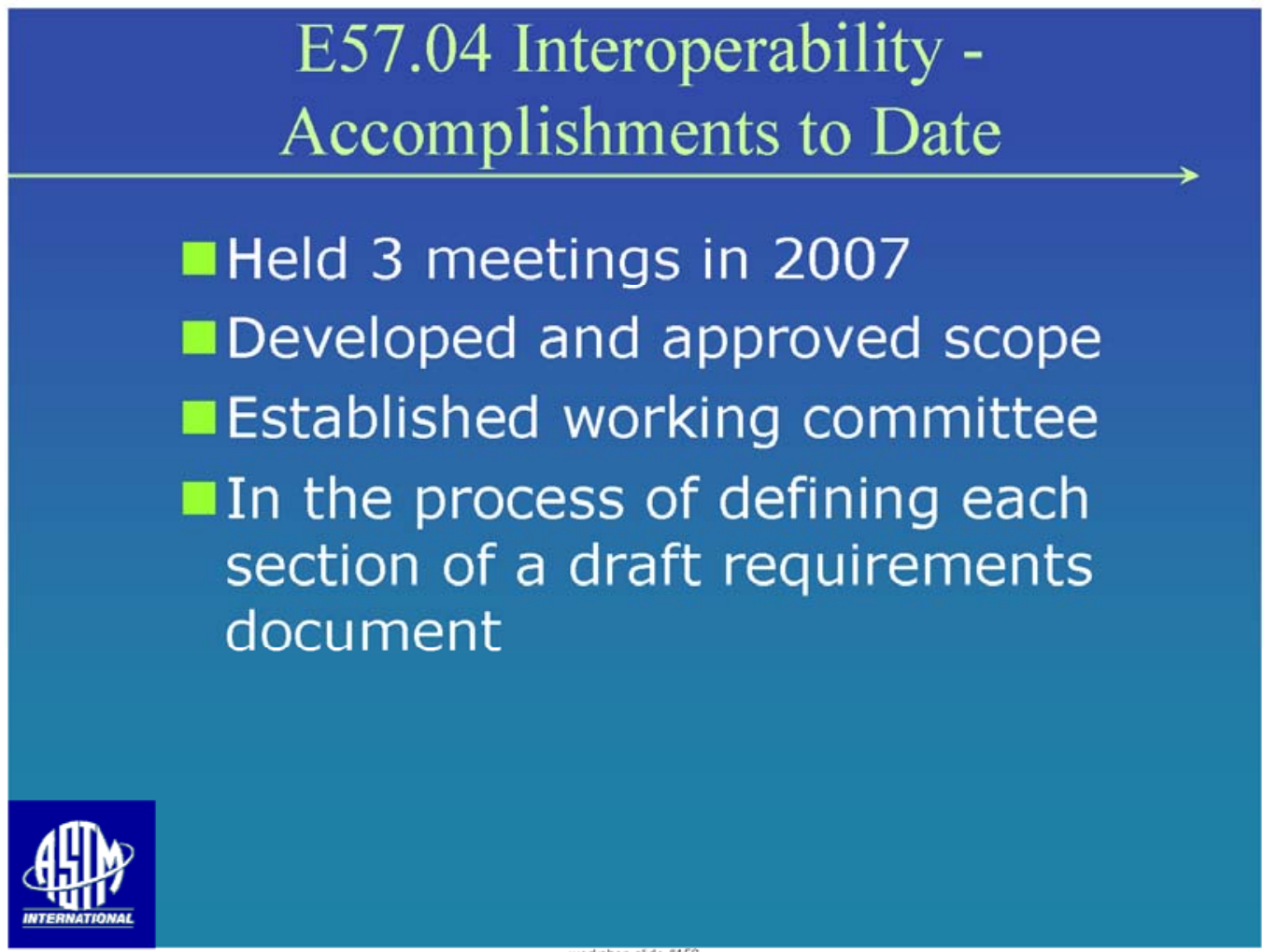




\section{E57.04 Data Interoperability - Goals}

Research existing data exchange formats such as LAS and XML

- Seek the input and involvement of all interested parties

Develop and proposed a Version 1 , common data exchange format that meets the needs of the industry within 12 months

\section{E57.04 Data Interoperability - 12 Month Work Plan}

- Develop requirements definitions by 9/1/2007

D Draft requirements document by $12 / 1 / 2007$

- Review and edit by $1 / 1 / 2008$

- Deliver final to ASTM January 2008 meeting

Develop draft of exchange format by $4 / 1 / 08$

- Obtain approval by ASTM summer meeting 2008

Interact with other standards organizations

Contact: Gene Roe, Gene.Roe@autodesk.com 


\section{E57 on 3D Imaging Systems}

-We need you (manufacturers and users) to come help E57 define a successful 3D Imaging standard.

口uestions? 


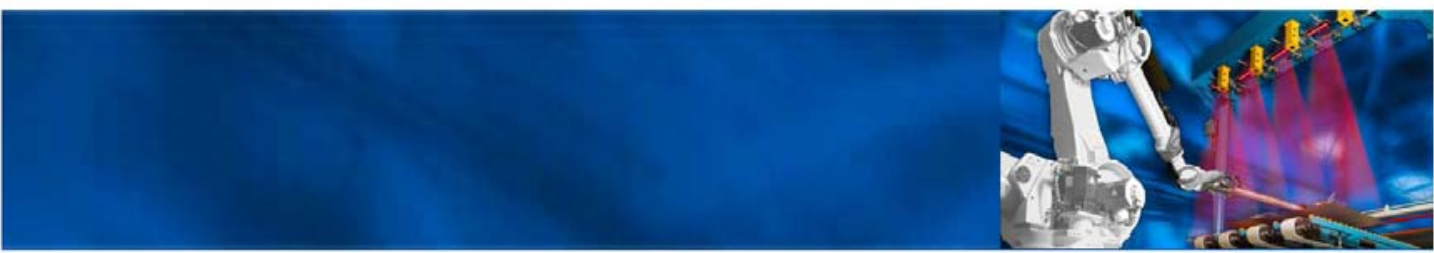

\section{D Vision, Robots and Movement - Putting it all Together}

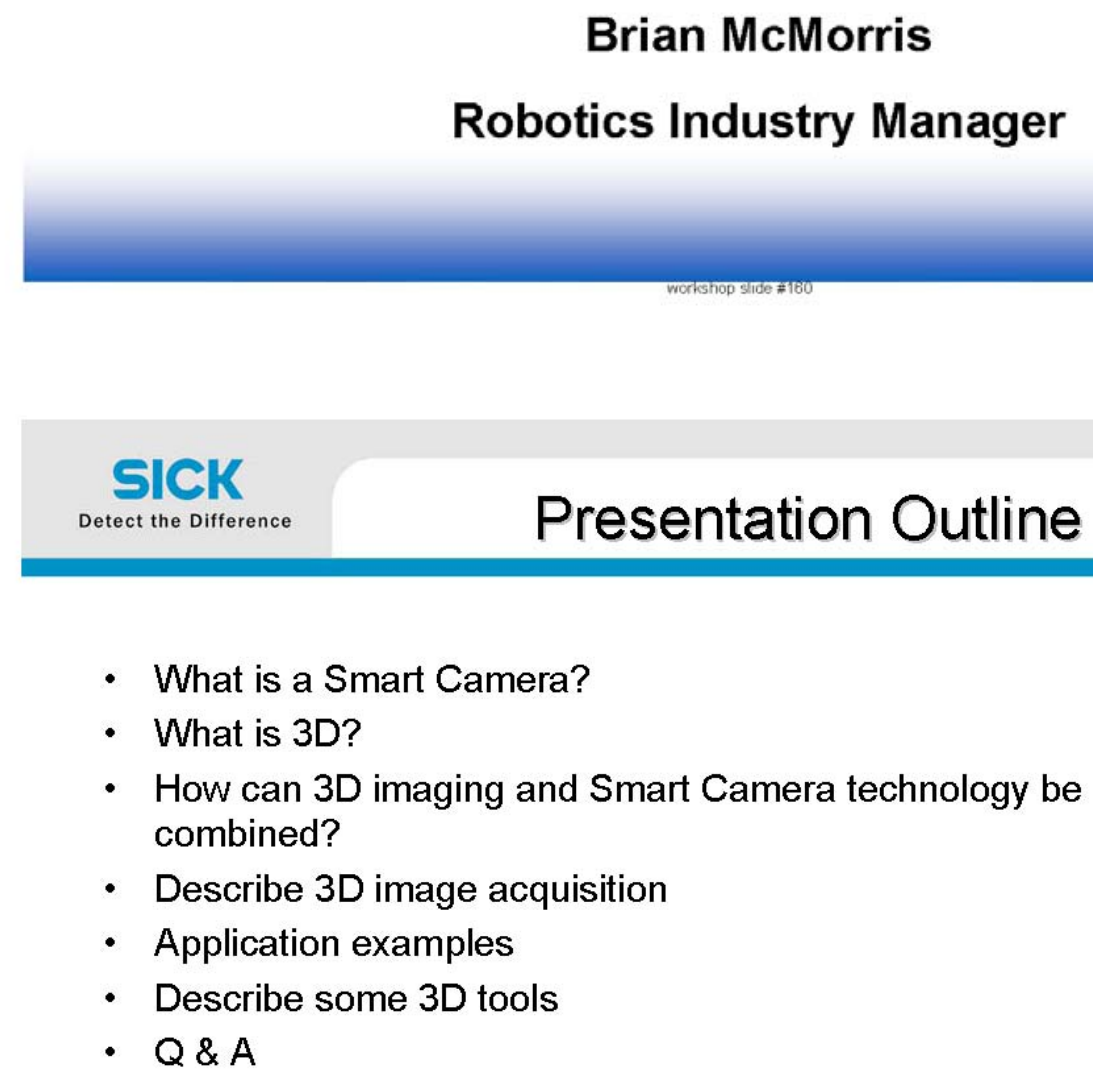




\section{What is a Smart Camera?}

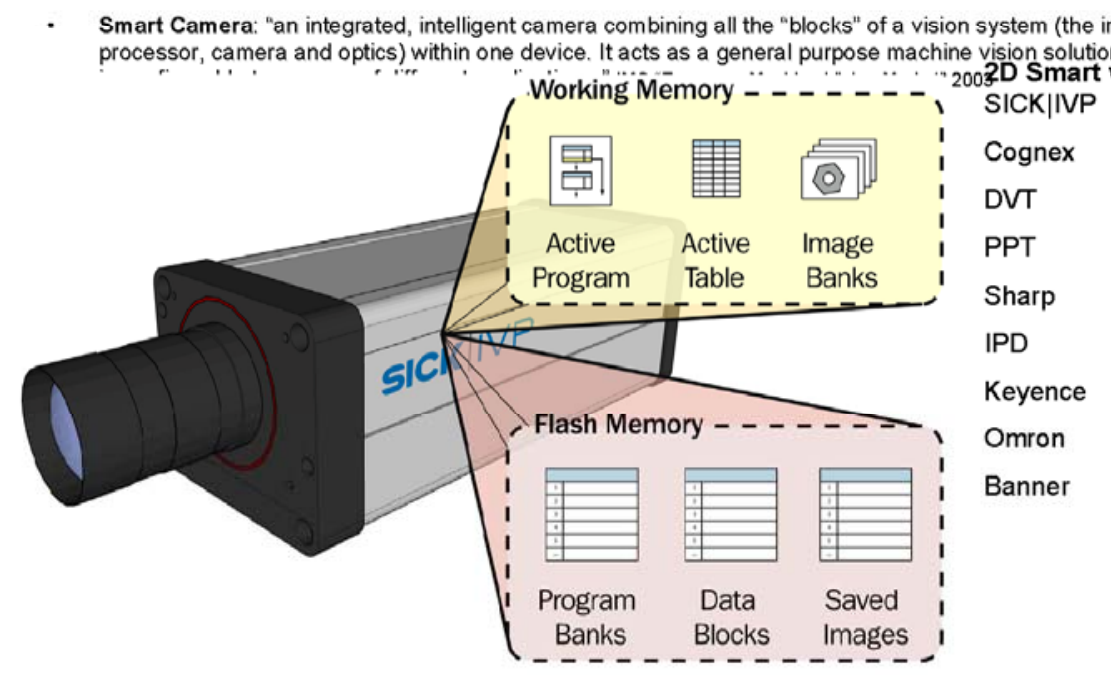

\section{Applications Difficult to Solve with 2D}

Missing objects
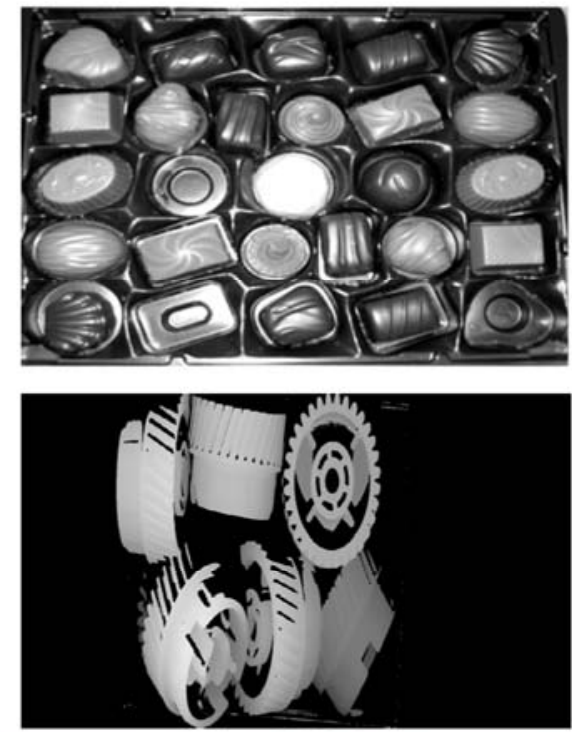

Low contrast applications

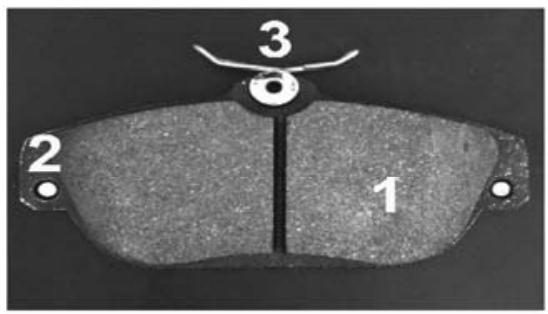

Bin picking random placement. X-X-Y-Z data required to pick object. 


\section{SICK}

Detect the Difference

\section{Encoder or Constant Speed}

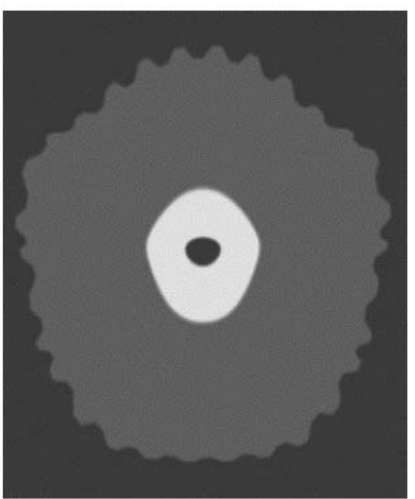

The velocity is uneven

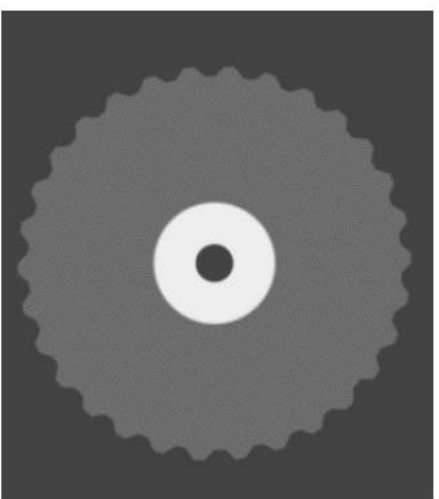

Constant velocity or encoder feedback

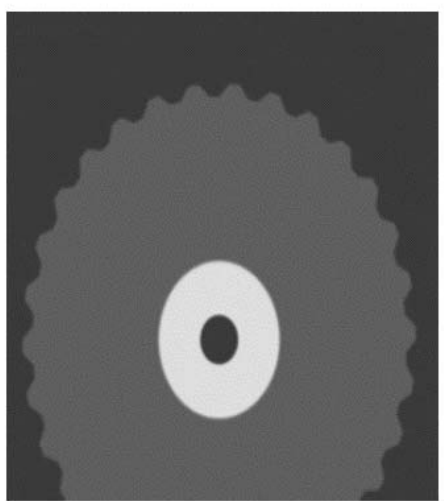

The velocity is too slow

\section{For robust implementations use encoders}

\section{$3 D+$ smart + tools}

We combined a Smart Camera, tools and 3D imagir Camera!!!

- 3D imaging (3D image capture, encoders, calibrated units -> 3D image and profiles)

- Smart (stand alone, general purpose vision processor)

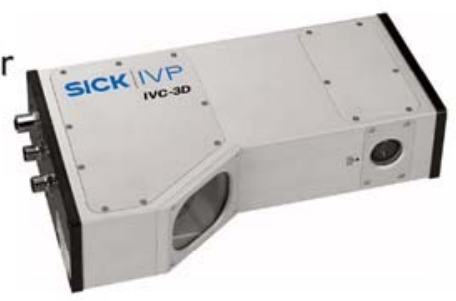

- Tools (well known 2D tools +3D specific tools for profile and 3D image processing)

Smart 3D technology vs. Smart gauge

Smart 3D technology vs. Streaming 3D device 
Acquisition of $3 \mathrm{D}+$ intensity using a single laser. Processing too complex for current 3D Smart technology.

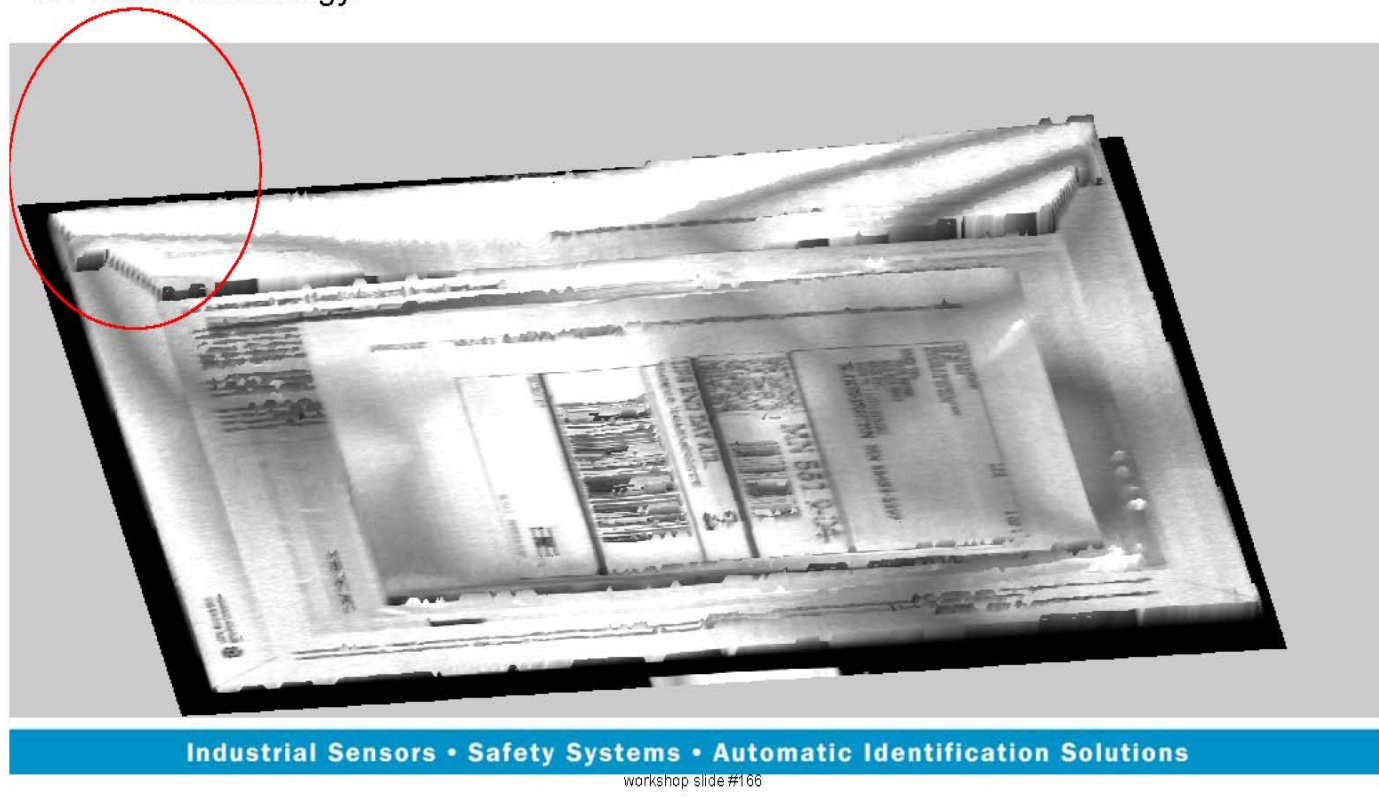

\section{SICK}

Detect the Difference

\section{Let's Solve a GOOD Application}

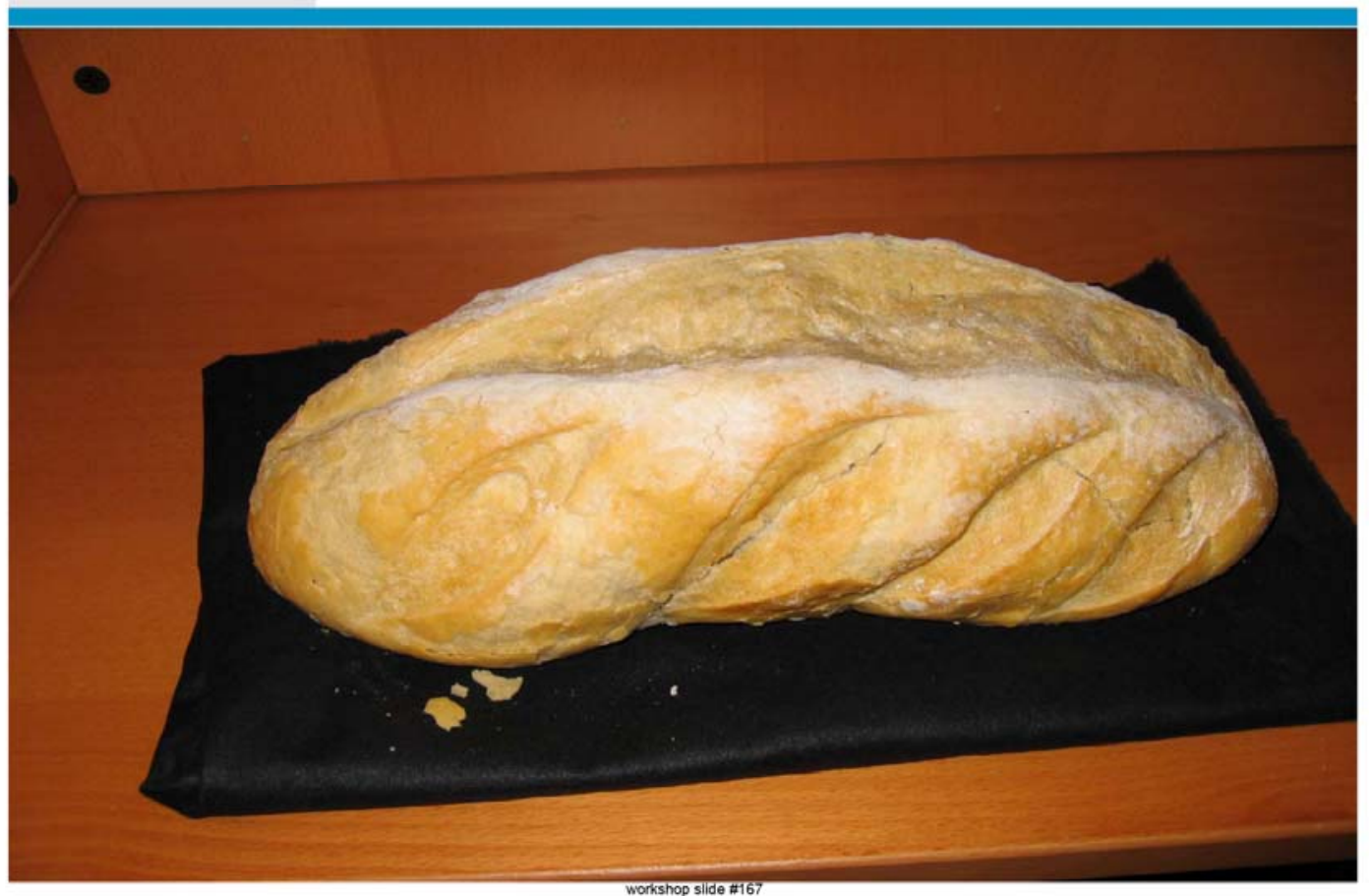

workshop side \#167 


\section{Slice the Loaf Using 3D Vision Tools}

- The number of slices depends on how hungry you are

- Volume of one slice $=$ Volume of loaf $/$ number of slices

- Set a thin ROI (one pixel high), moving ROI at the beginning of the loaf and set the accumulated volume to 0 .

For number of slices:

- Calculate the volume of the thin part of the loaf that is inside the $\mathrm{ROI}$ and add this volume to the accumulated volume.

- Move the ROI one pixel at a time and add the volume inside the $\mathrm{ROI}$ to the accumulated volume.

- When the accumulated volume becomes larger than the desired volume of a slice, cut the loaf (or just mark the location) and reset the accumulated volume.

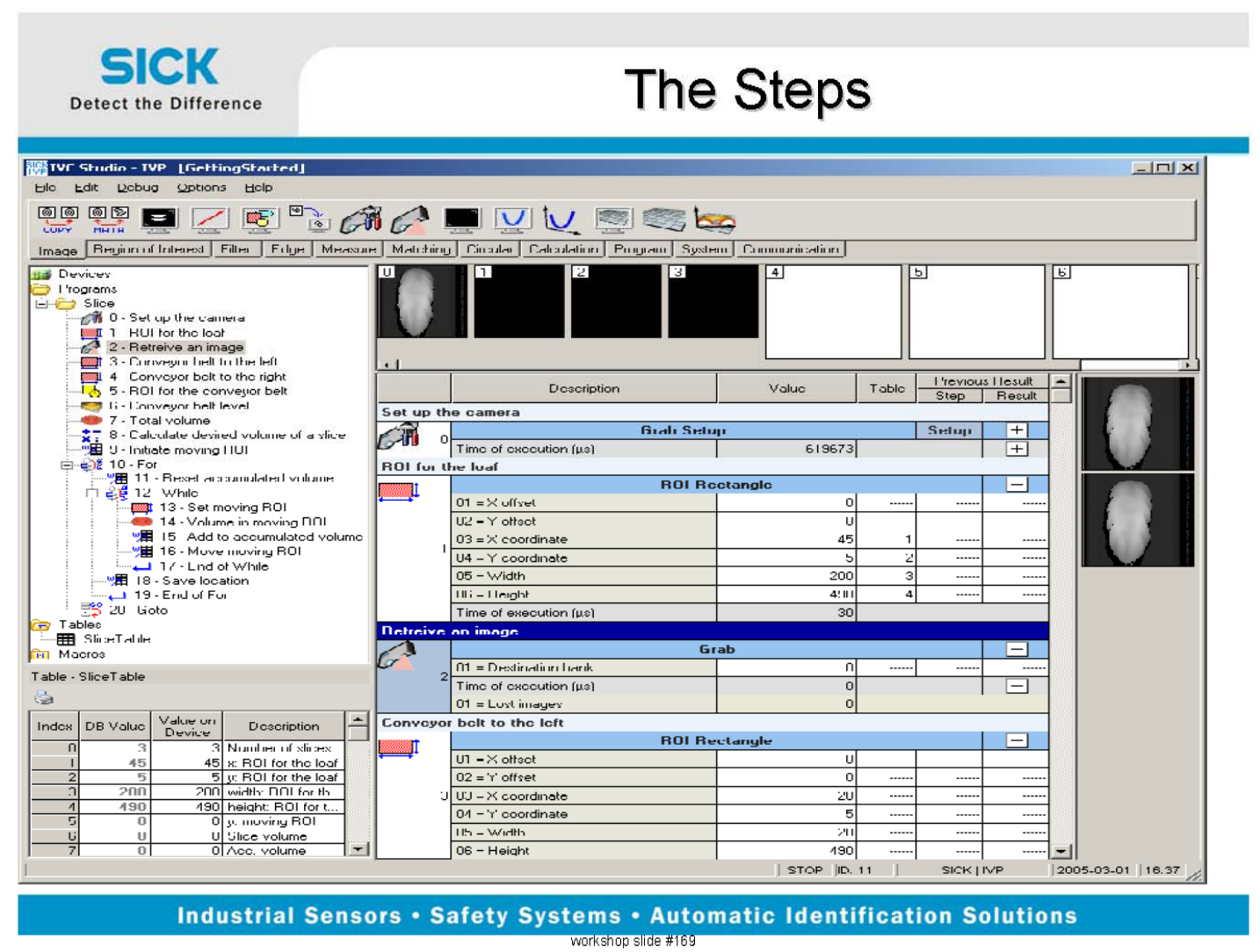




\section{Dedicated 3D Image Processing Tools}

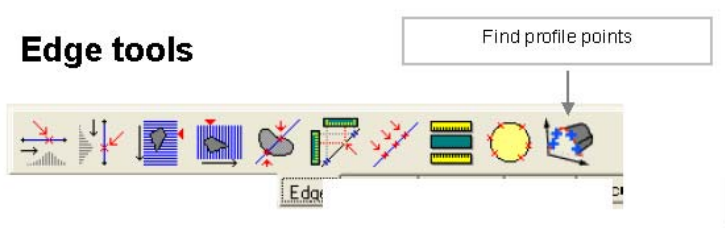

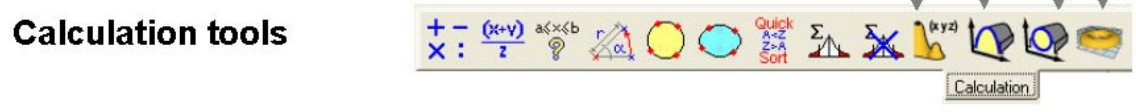
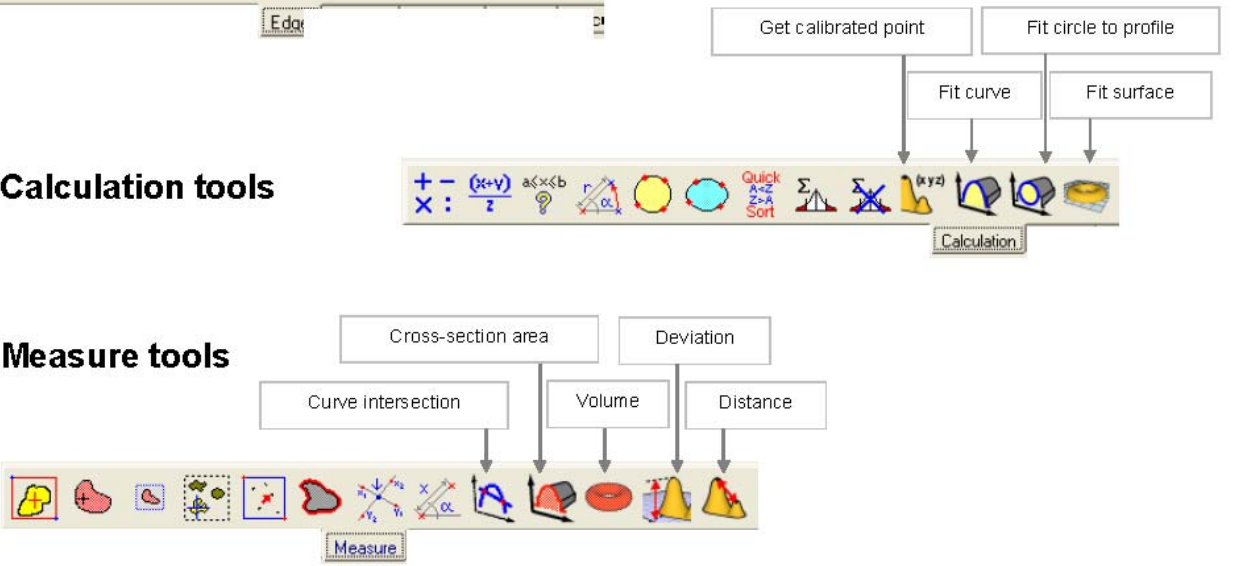

\section{Dedicated 3D Image Processing Tools}

\section{Image tools}

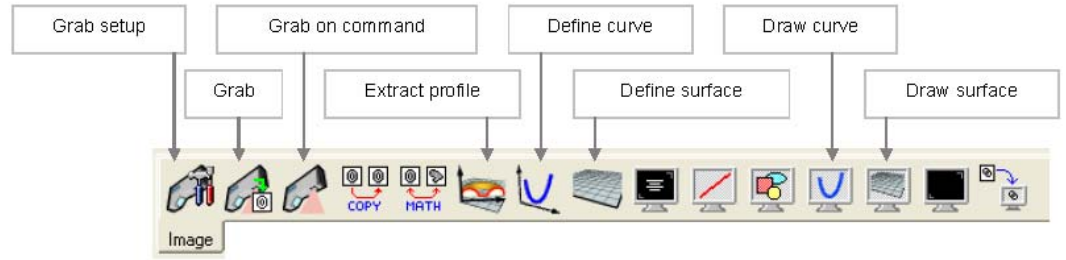

ROI tools

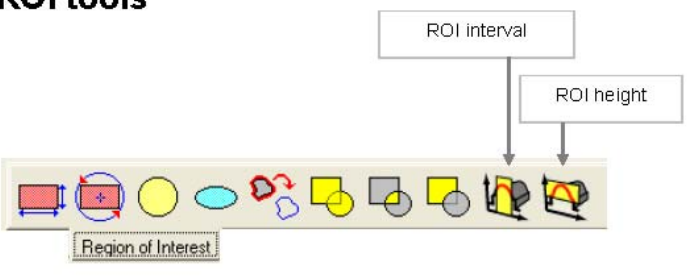

Filter tools

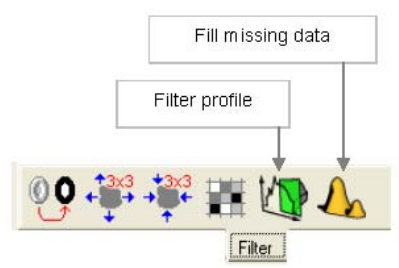




\section{SICK}

Detect the Difference

\section{Brake Pad Inspection}

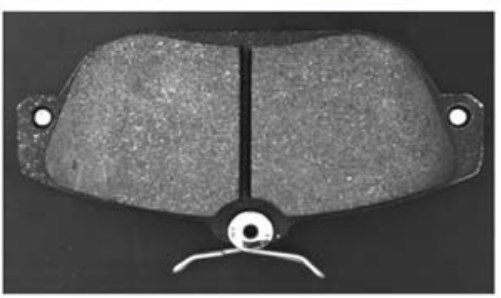

i4t 0 - If Error Goto

Co 1 - Initialization

2-Wait for trigger to go high

- 3 - Reset the first image bank

wh 4 - Reset Fail flag

Q? 5 - Start an image aquisition

- 6 - Reset all other image banks

3. 7 - Place the new image in bank0

8 - Detect Surface Cavity

마 9 -Define the entire image bank

10 - Define the base surface

19. 11 - Draw the base surface

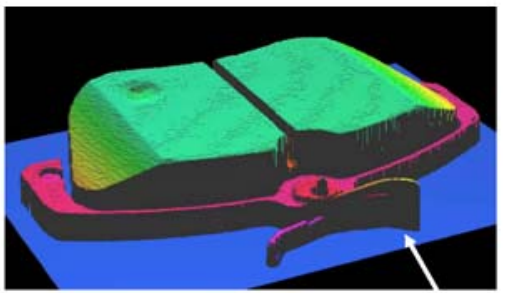

uㅐ 12 - Add all pixels higher than the base surface

[6] 13 - Find one point that will allways be on top of the object

D 14 - Find the outline of the top of the object

of 15 - Define a Region of Interest inside the top of the object

16 - Fit a surface to the top of the object within that ROI

In 17 - Find the height of that surface

4. 18 - Locate the surface cavity within the ROI

Q5 19 - If then Goto

므 20 - Add Text

"困 21 - Set Fail flag

+ 792 -Detecting cable

\$I 29 - Jump on Detected Defects

How is the spring angle and height measured with 3D tools?

[ 30 - Print Pass Text

[क] 31 - Display

\% 32 - Read Input

\section{Industrial Configurations for 3D Smart}

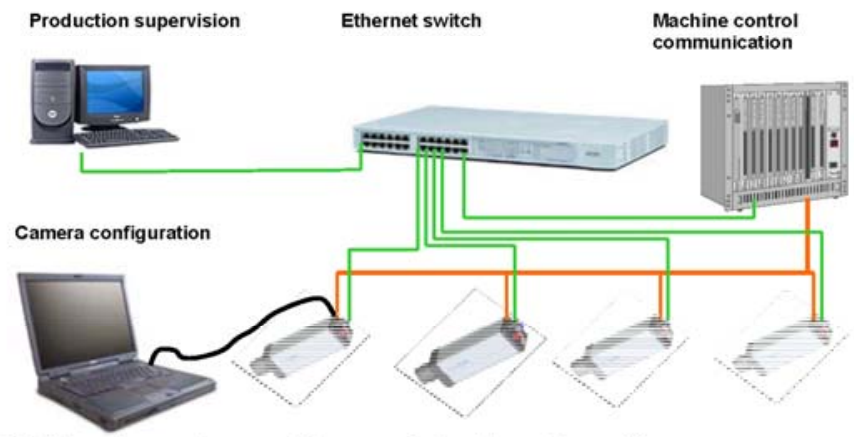

- $3 D$ and 2D Smart can be used in a variety of configurations:

- Stand-alone single camera unit

- Stand-alone multiple camera unit

- Managed by a control system

- Monitored by a PC 
A 3D image shows the topology of an object, or the distance from the bottom (or reference plane) to a point on the surface of the object. The brighter a pixel is in the image, the higher up that point is on the object.

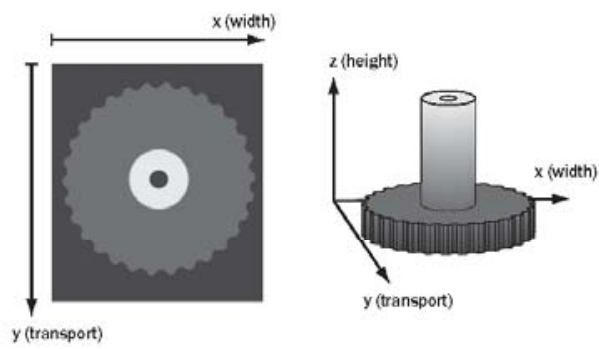

SICK

Detect the Difference

\section{Finding the Volume}

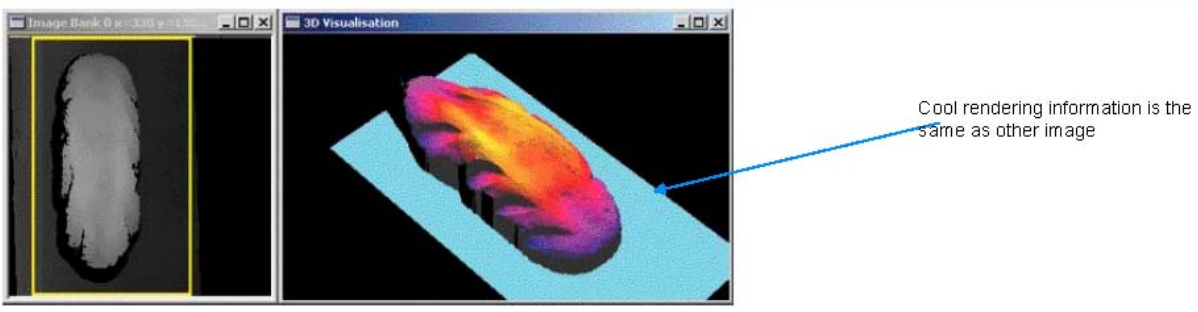

RoI Rectangle (find the bread)

The rectangle that is the region of interest (ROI) in which we expect to find the loaf or the conveyor belt.

- Rol Union (find the conveyer)

- Two ROls join. An ROI is specified by referring to the program step in which the ROI is created.

- Fit Surface (find the crust)

An inage bank containing a 3D image, an ROI, and the type of surface to fit to the part of the $3 \mathrm{D}$ image inside the ROI.

- Volume (calculate the bread volume)

- An image bank containing a 3D image, an ROl and a surface to use as zero-level when calculating the volume inside the ROI. Anything in the image below the zero-level is ignored when calculating the volume.

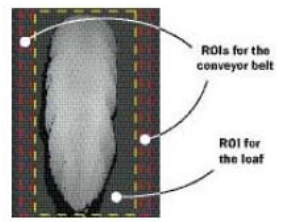

Industrial Sensors - Safety Systems - Automatic Identification Solutions

Workshop side \# 175 


\section{SICK}

Detect the Difference

\section{IVC-3D Smart Applications}
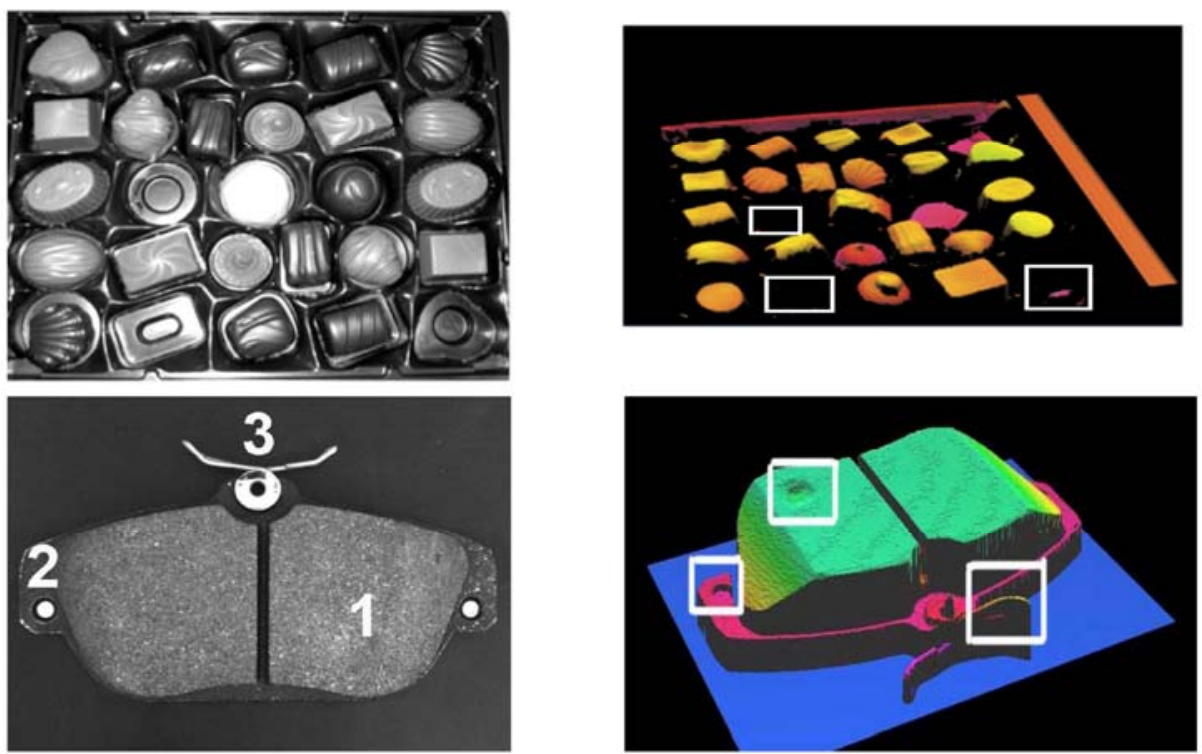

\section{Ideal 3D Applications for SICK Vision}

- Weld Seam and Glue Bead Inspection: Vision Guided Robotics

- Part Picking: Random Orientation in Bin, Auto Racking, Conveyor, Chain Hangar

- Machined and cast parts with non-square edges (poor shadowing)

- Washers (not flat), extrusions, metal stamping (no contrast)

- Palletizing, Depalletizing, Stacking, Case Packing

- Machine Tending: Load and Unload : Vision Guided Robotics

- Low contrast imaging applications: rubber, plastic, asphalt, baked goods

- All types of volume surface feature applications:

- Metal machined and welded parts

- High speed surface inspection, e.g. In-motion Railroad rails and rail beds, highway surface quality (mapping potholes and cracks)

- Packaging (confirm integrity of boxes, presence/absence of product)

- Baking and cookie inspection

- Tires, gaskets, automotive trim parts (low contrast, non-squared edges)

- Pharmaceutical applications (blister and fill levels)

- Turbine blade inspections

- Size distribution by volumetric calculation (single and multiple objects) 


\section{Robot Auto Industry Applications}

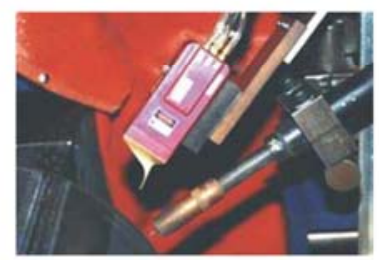

Welding

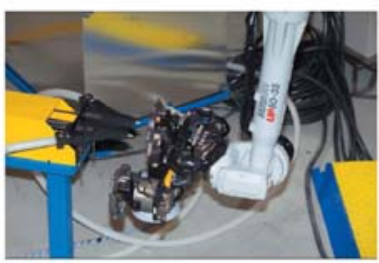

Assembly

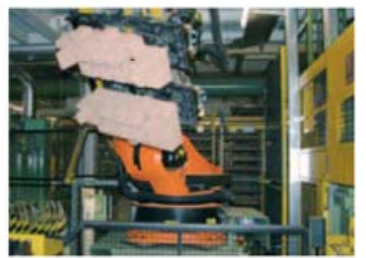

Handling

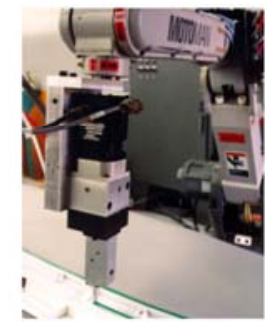

Glue Dispensing

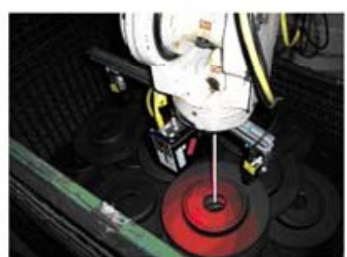

Machining

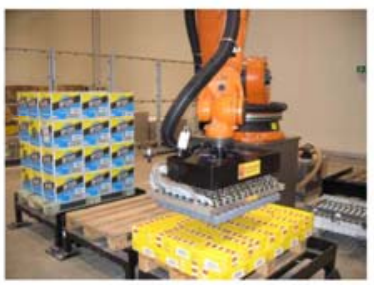

Picking / Palletizing

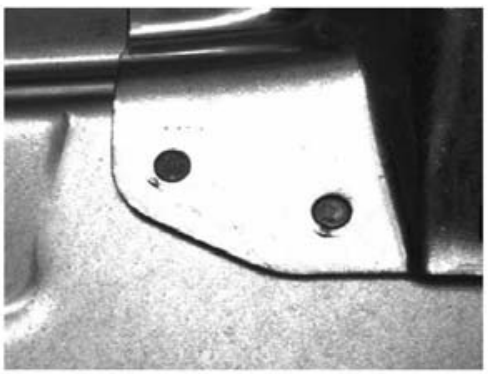

- Inspection for weld presence and position using $2 \mathrm{D}$ vision

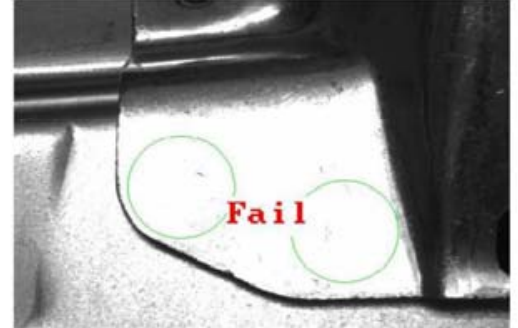

Found welds $=0$ Industrial Sensors - Safety Systems - Automatic Identification Solutions

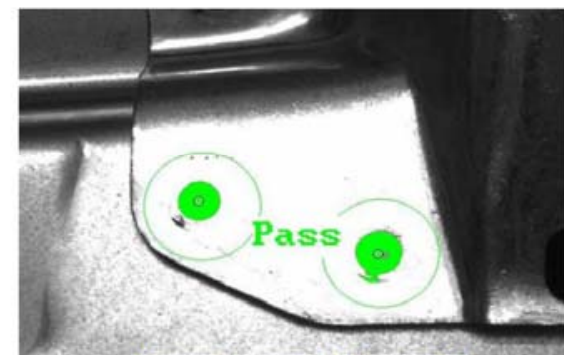

Found welds $=2$ 


\section{What is 3D Profiling?}

\section{Detection, Positioning and Inspection with 3D Shape}

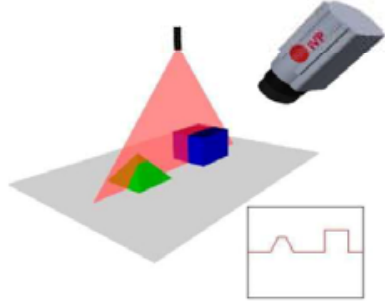

Which object is on top?
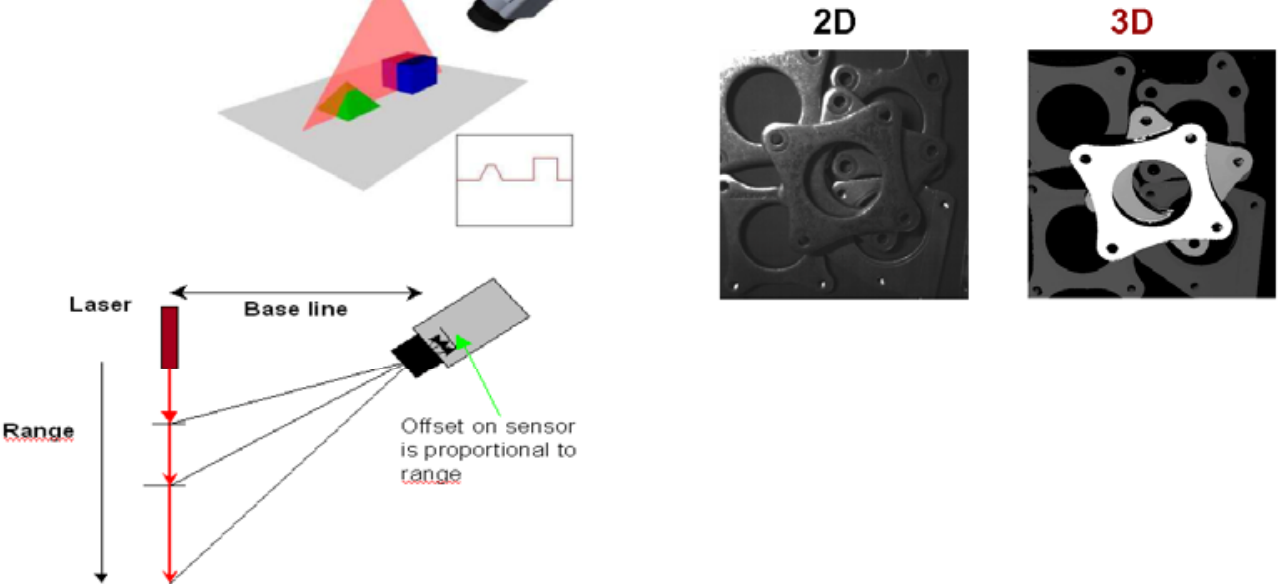

\section{D Vision Buzz Words}

- Occlusion: Laser and lens

- Laser class: Class II eye safety. Can affect frame rate

- Resolution: (for guidance only)

- Cross (X), Width/number of pixels

- Example $500 \mathrm{~mm}$ wide belt, a sample every $0.5 \mathrm{~mm}$

- Movement direction $(Y)$ Velocity/ frame rate

- Example $1 \mathrm{~m} / \mathrm{s} / 5000->5$ profile $/ \mathrm{mm}$-> 500 profiles for $50 \mathrm{~mm}$ long object

- Height (Z): Depends of FOV (Sub pixel techniques)

- 1 inch $\sim 5$ microns ( 1 inch FOV (width) is possible to achieve about 5 microns $Z$ resolution

- 5 inches $~ 1 / 1000$ inch

- 30 inches $~ 1 / 100$ inch

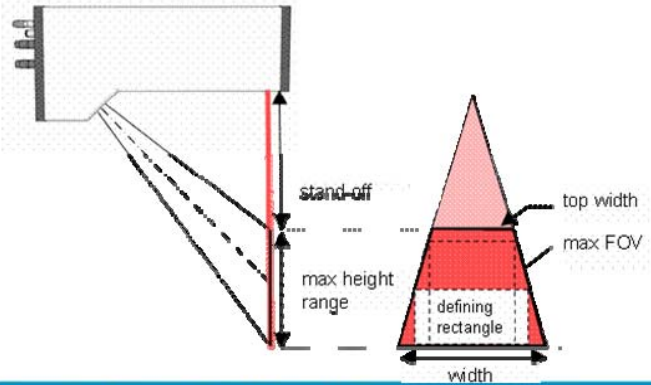




\section{D Calibration Basics}
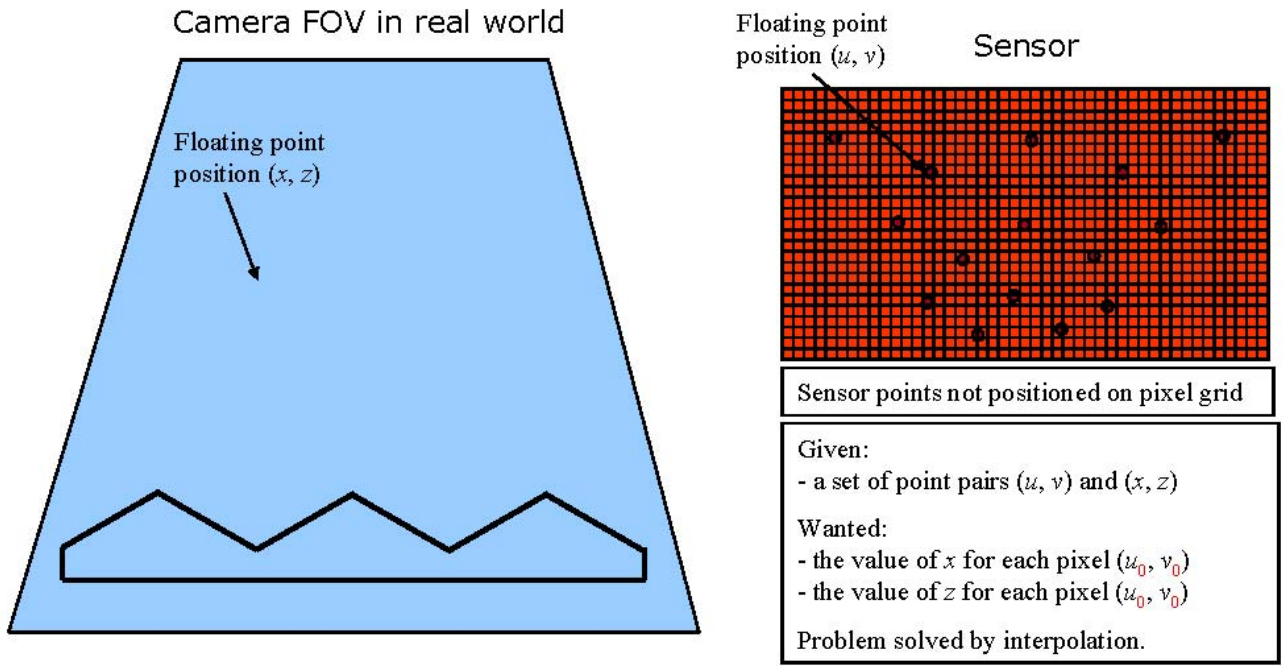

\section{Local Surface Fitting Model Calibration}

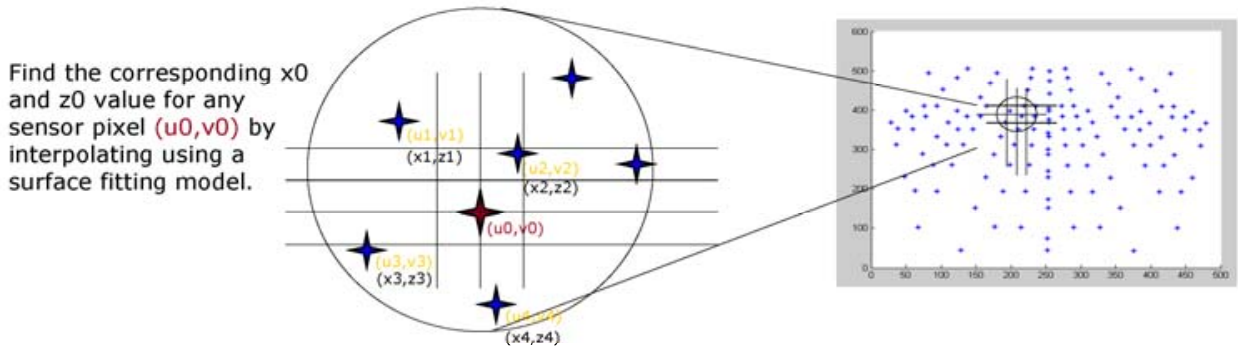

The surface fitting model should interpolate from the 6-10 nearest neighbours to get both the $x$ and $z$

Generate 2 look-up tables. value.
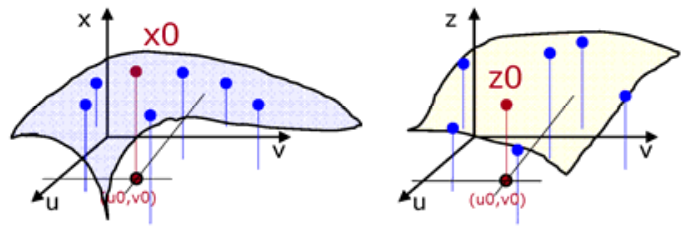

One for $x$ and one for $z$

values for each sensor

pixel.

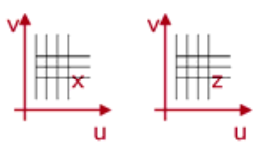

Industrial Sensors - Safety Systems - Automatic Identification Solutions 


\section{SICK}

Detect the Difference

\section{Correcting for Effects of Perspective}

\section{Non-uniform Samples Calibration Along X}
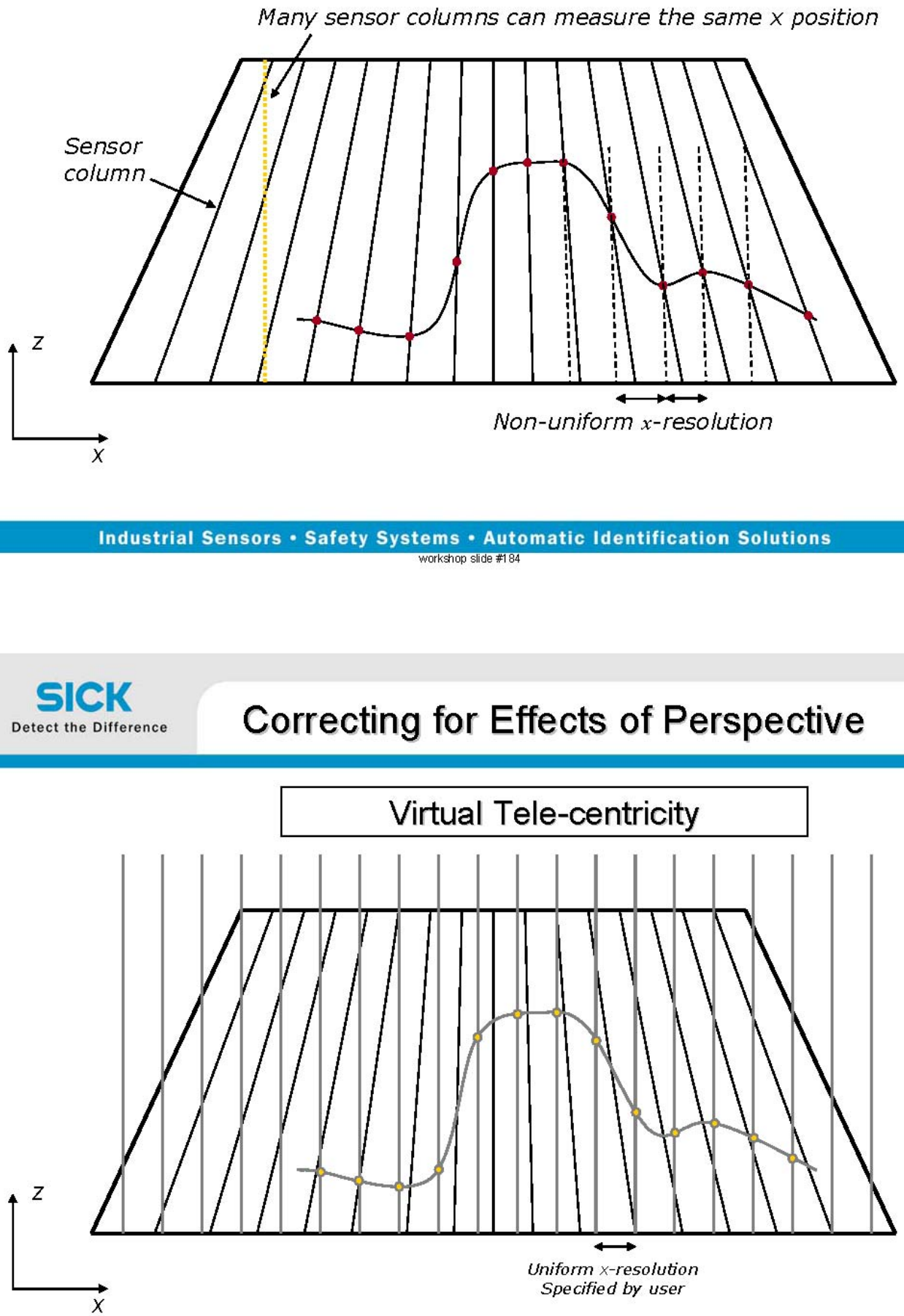


\section{Delta Robot Bread Packaging}

- A 3D camera locates bread buns for packaging by a delta robot

- Faulty buns are rejected

- Stand-alone operation, no PC needed for image processing

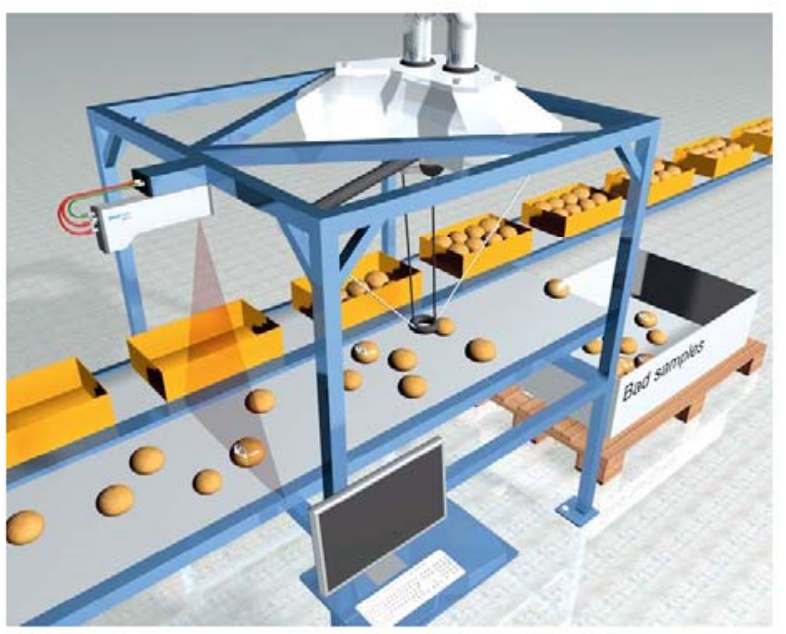

\section{Camera Robot Calibration}

- The 3D camera delivers results in robot coordinates!

- Calibration method

- Mount the camera

- Scan a calibration object. The camera measures key positions

- Point out the key positions with the robot

- The camera calculates the coordinate transformation
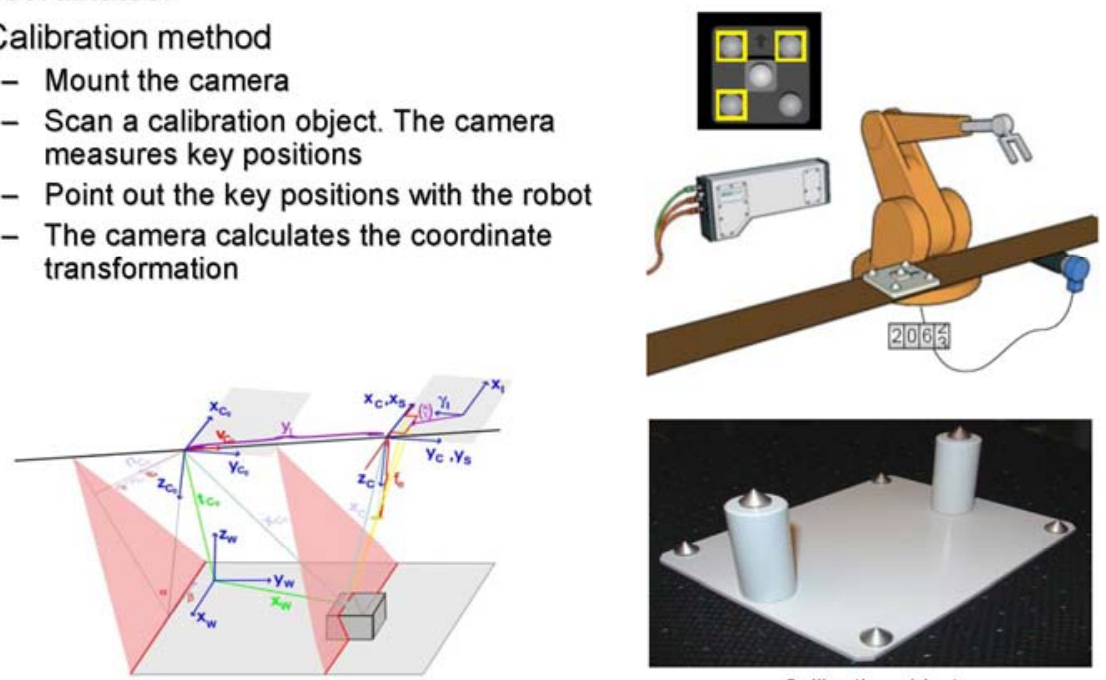

Calibration object 


\section{SICK}

Detect the Difference

\section{Conveyer and Robot Calibration}

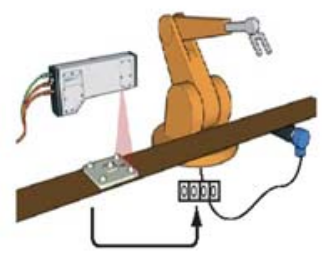

3

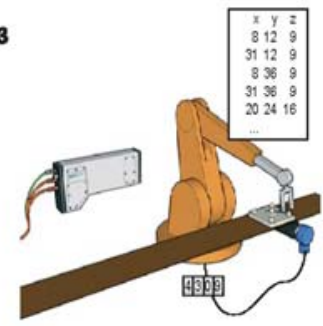

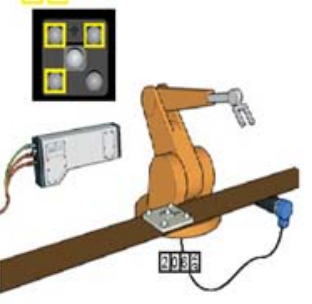

4

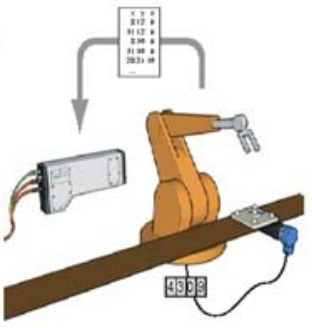

1. Grab a 3D image of the reference object. The Smart Camera sends a trigger pulse to the robot

2. Mark the reference points in the $3 \mathrm{D}$ image and move the object to the robot. The robot uses an encoder to keep track of the object's movement.

3. Get the robot's coordinates for the reference points.

4. Import the robot's coordinates if necessary, adjusted by the movement - to the Smart Camera and calculate the transform.

- Allows picking of complex products

- 3D shape is often much more important than $2 \mathrm{D}$ pattern when picking up objects

- Does not require unique features for part location

- Contrast-independent inspections

- Dark products on dark conveyor

- Color-insensitive

- Insensitive to dirt or patterns

- Robots often handle products before their appearance has been finalized (e.g. painting)

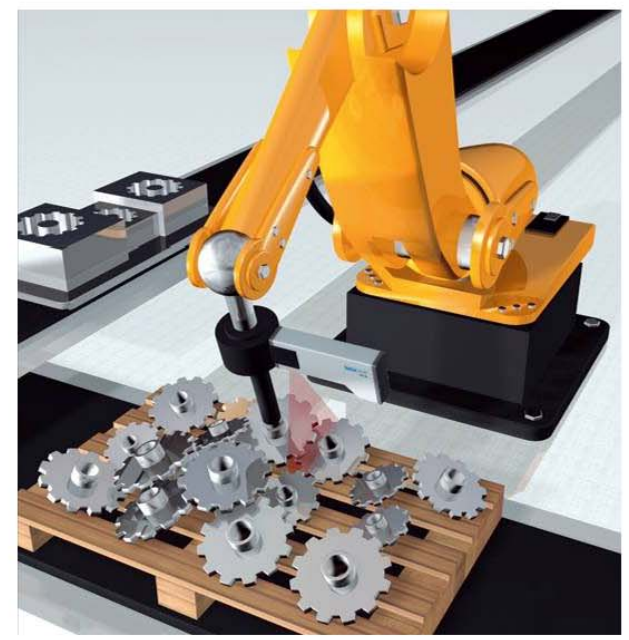




\section{Bin Picking}

Some of the factors that determine bin picking complexity

- Part: Shape, surface features, material, size, fragility

- Presentation: Bin with/without sides, random, matrix, stacked parts, layers

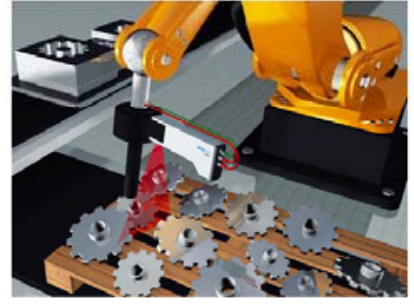

- Required throughput (parts/second): Robot movement capabilities, part scanning

- Precision: System requirements for pick point precision and the ramifications for calibration, transformation and robot position

- Other: Collision avoidance requirements, robot interface

\section{SICK}

Detect the Difference

\section{D Robot Calibration, similar to 2D}

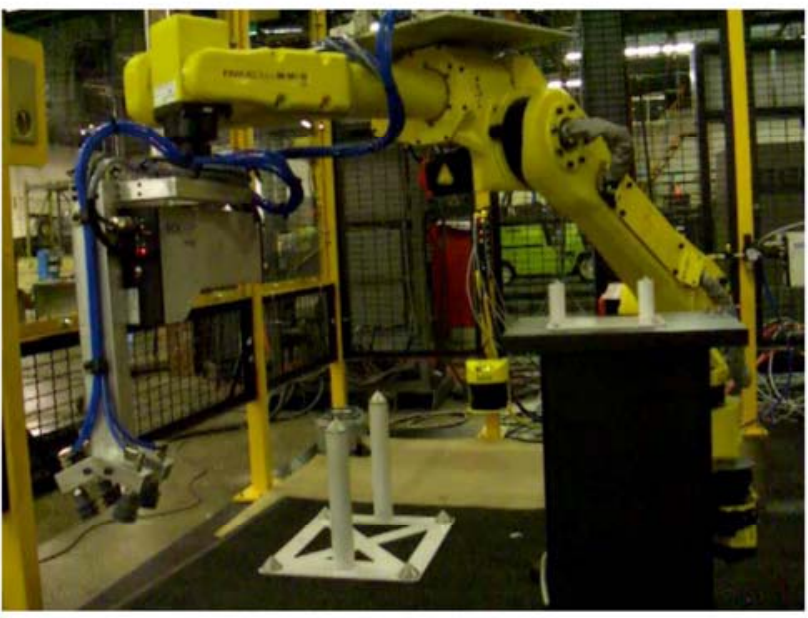

Calibrating robot with SICK 3D reference tool

Transforming vision system coordinates to robot coordinates

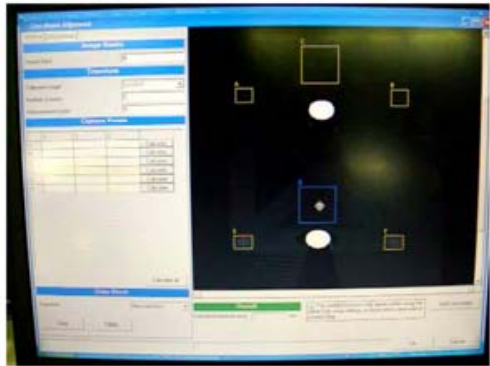




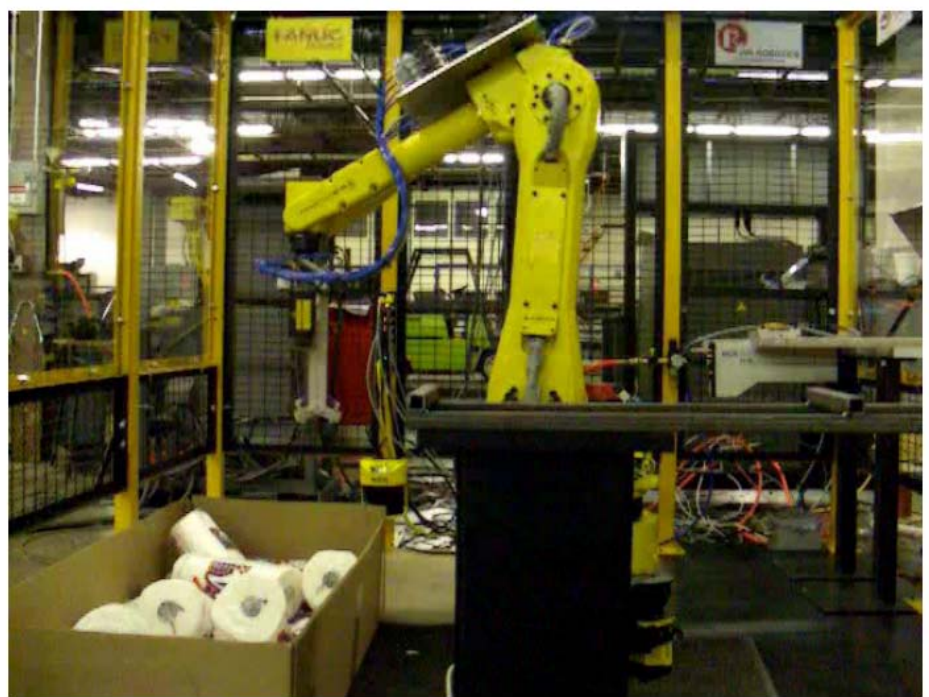

Example of random bin picking using Smart Camera (IVC-3D-200); no fixtures and non-uniform objects to pick

\section{Random Bin Picking is Here}

- Acquire 3D image of objects

- Report coordinates and orientation in 6 DOF (Degrees of Freedom) to the robot controller

- Robot picks the approriate object

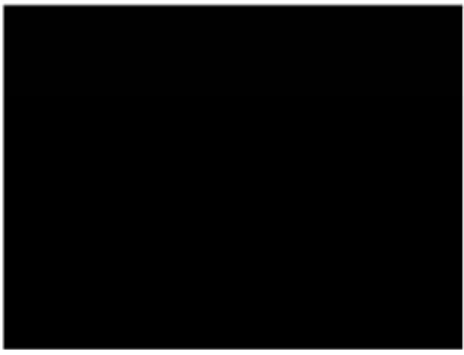

Fraunhofer Institute IPA \& RoboMan (using IVC Ranger camera)

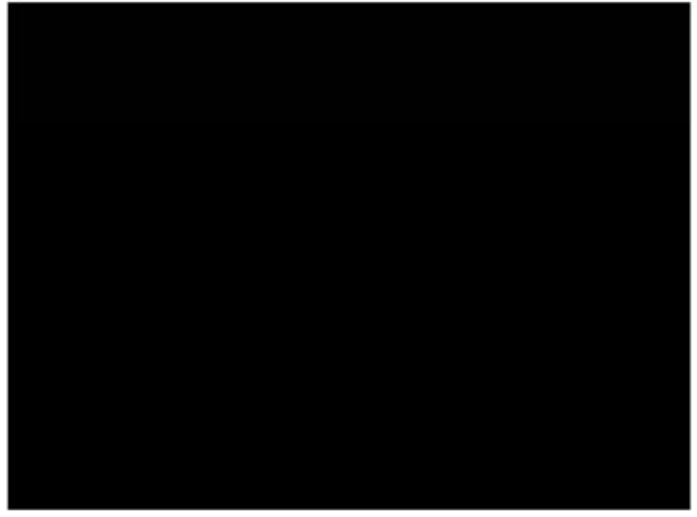

Master Automation 


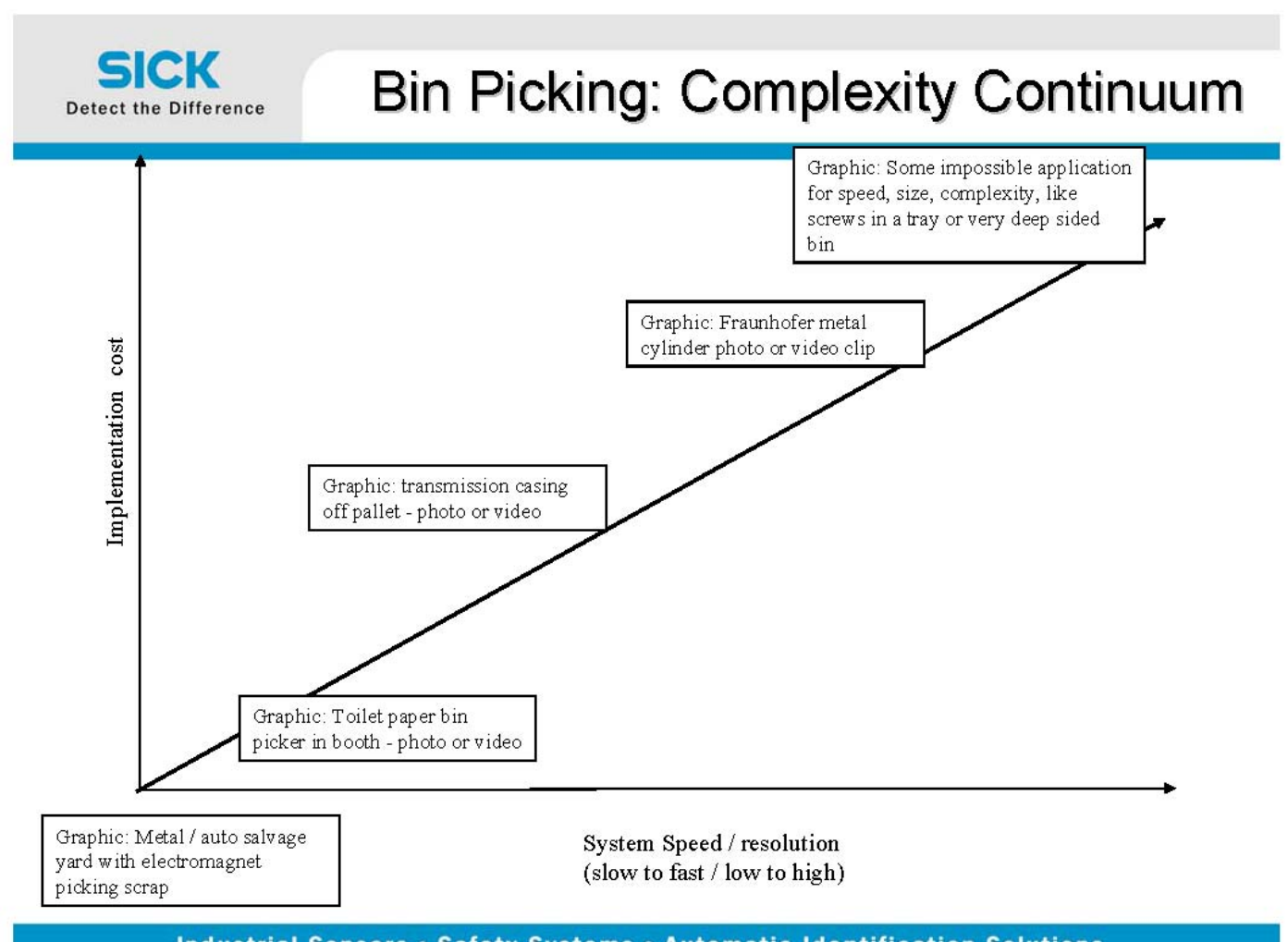

\section{Painting of Car Bodies: VGR}

- The 3D shape of the car body is measured and reported to the robot controller

- Optimal paint-stroke pattern is calculated

- Painting starts
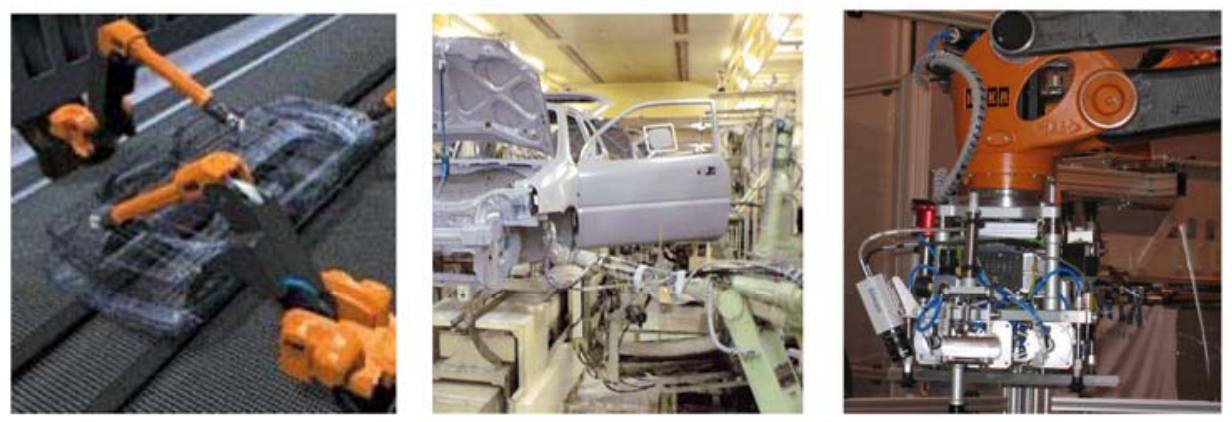
- A 3D system is used for contrast-independent palletizing

- Packages are located on the conveyor, then placed correctly on pallets
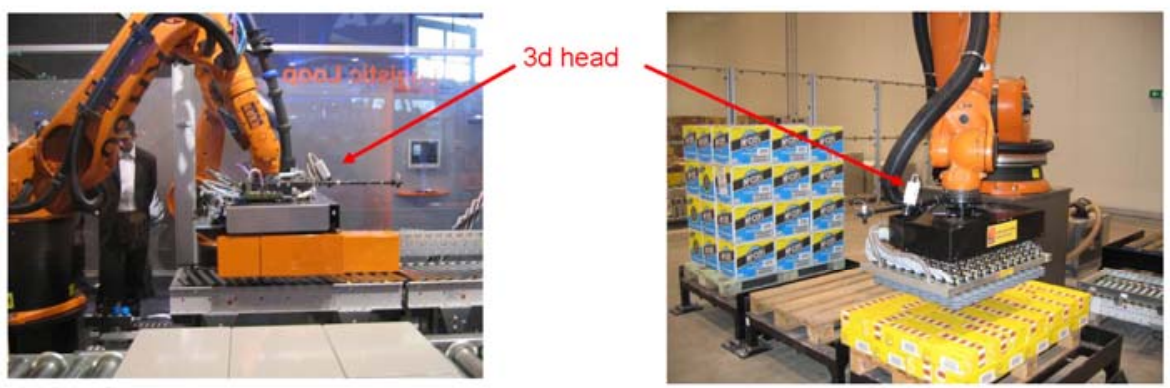

\section{Crate Handling}

- A 3D camera scans the crate and locates all bottle caps

- Contrast-
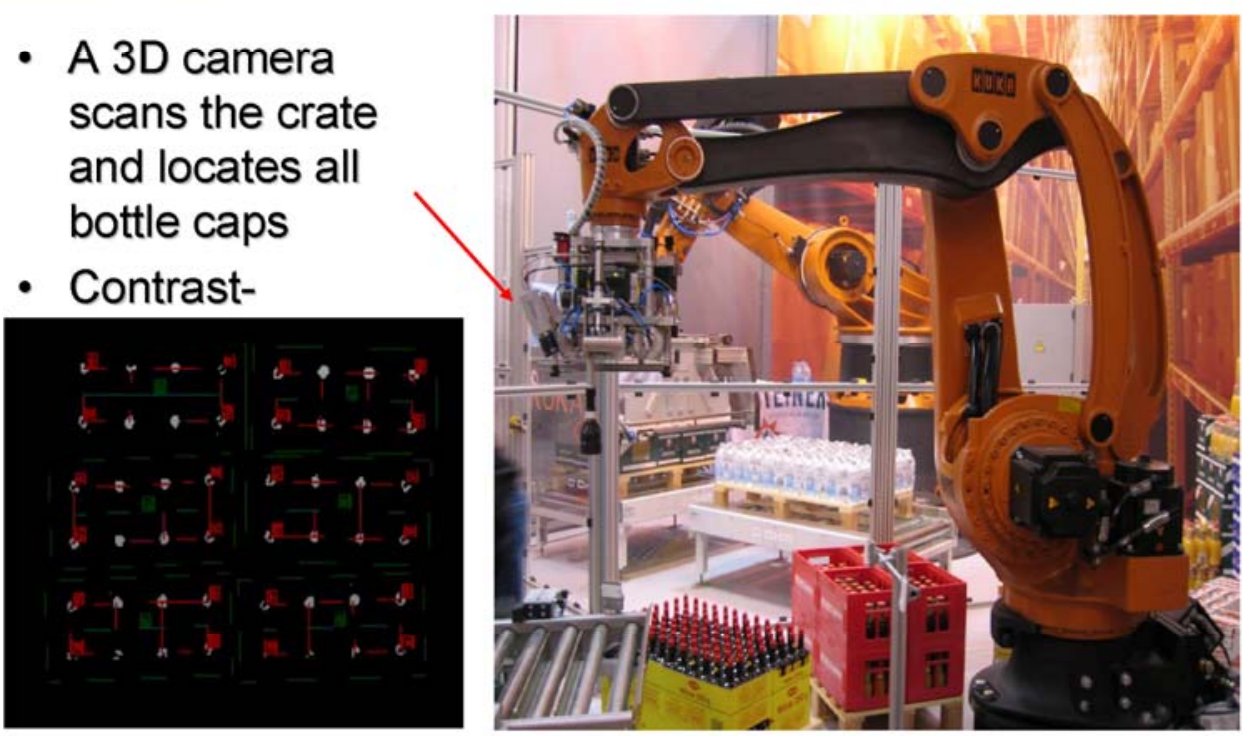


\section{Depalletizing in Automotive}

- A 3D camera is used for exact location of a gear box part on a pallet

-
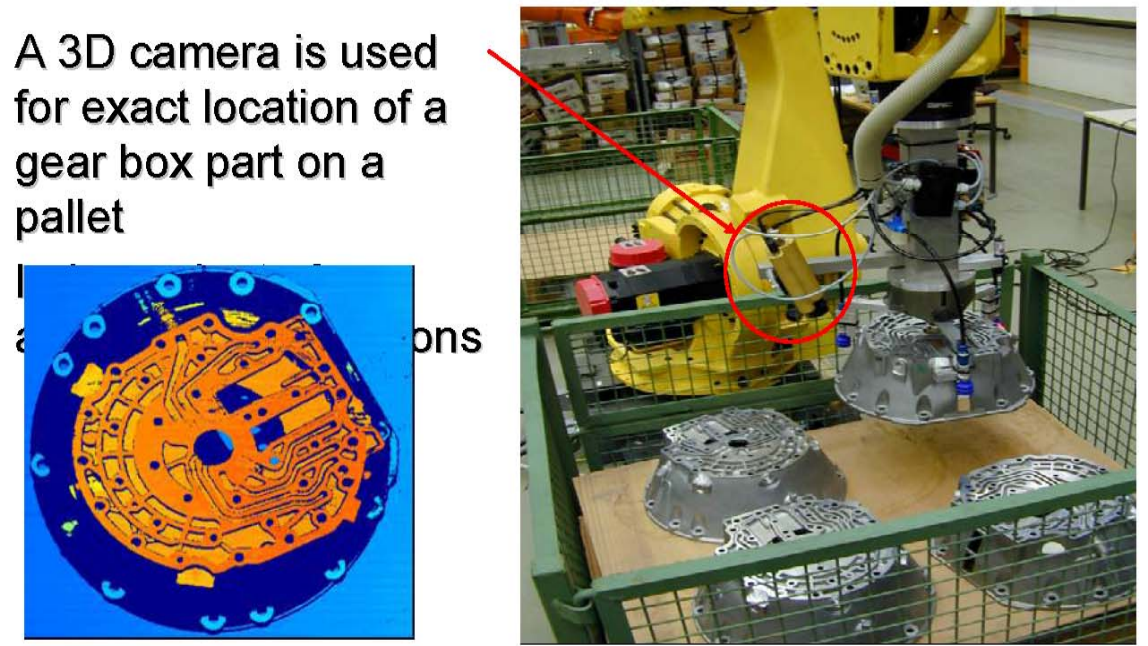

SICK

Detect the Difference

\section{Glue Dispensing/Weld Seam}

Direct robot control from 3D smart cameras (no PC or remote controller needed)

- Monitor volume, shape, height and width of weld/glue

- Feedback for dosage control and weld parameters

-Smart 3D device will control robot directly via ethernet/serial

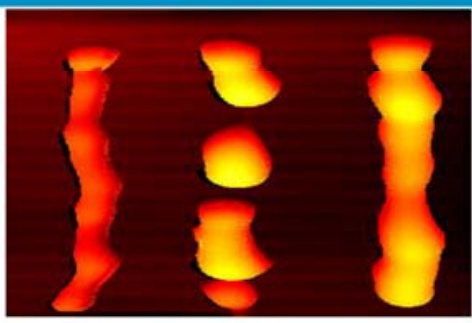

Glue beads

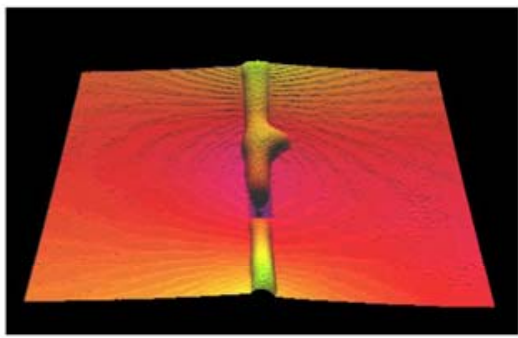

Weld seam 


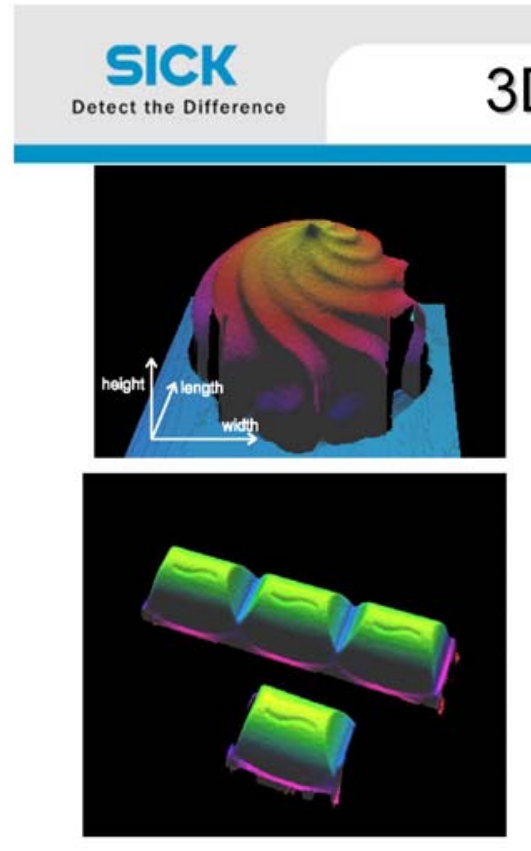

More chocolate

\section{D Images of Chocolates}

3D image of chocolate

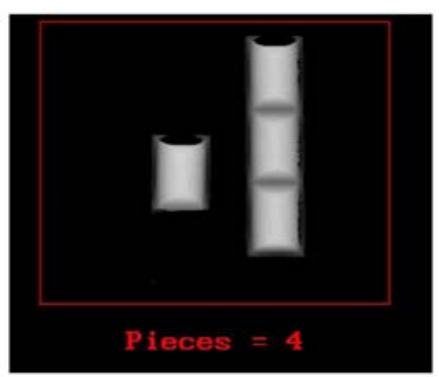

3D Smart Output-> OK product. Four pieces of perfectly shaped chocolate

\section{SICK}

Detect the Difference

\section{De-palletizing}

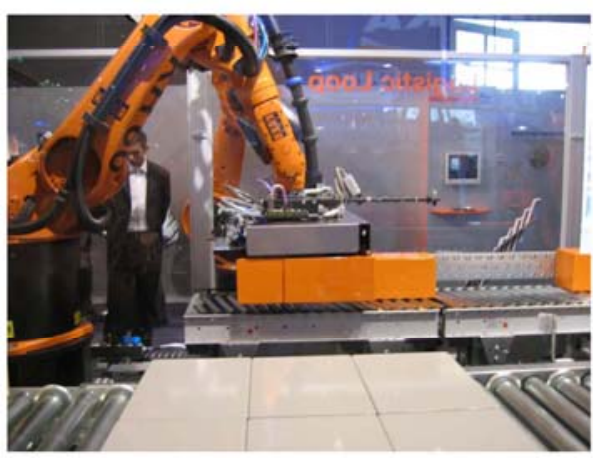

Handling non-contrast items

Handling various contrast items

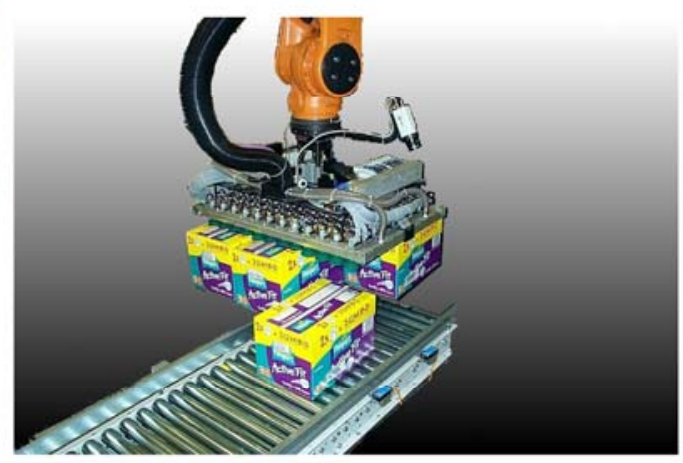

J

KUKA Courtesy of KUKA 

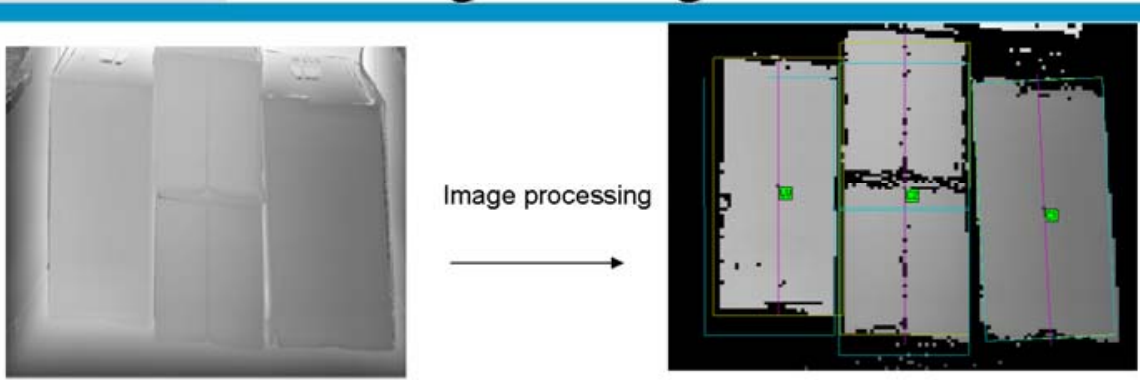

Filtering combinations of erosion and dilation (opening and closing)

Blob search with adaptive 3D height gray values

$3 \mathrm{D}$ gray values limits based on histogram data

Found blobs (connected areas of specific 3D gray height value) are evaluated regarding their length and width to match the searched articles

Blob matching

Unidentified blobs are reevaluated to match double sized packages 


\section{Profile Scanner}
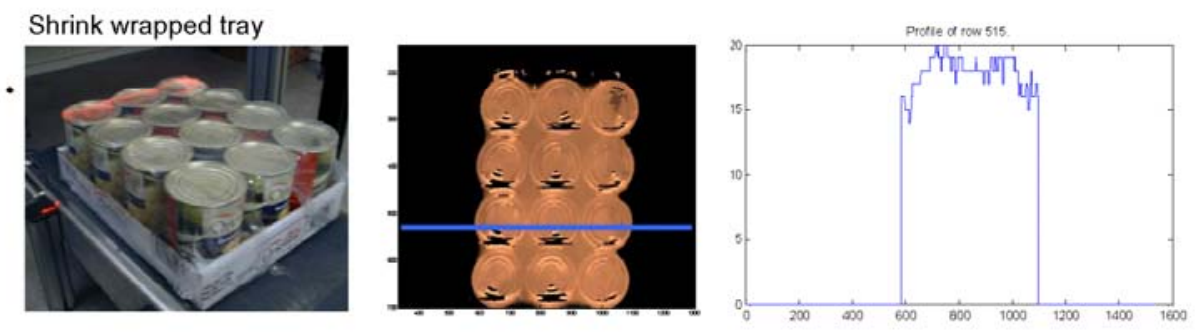

Open tray
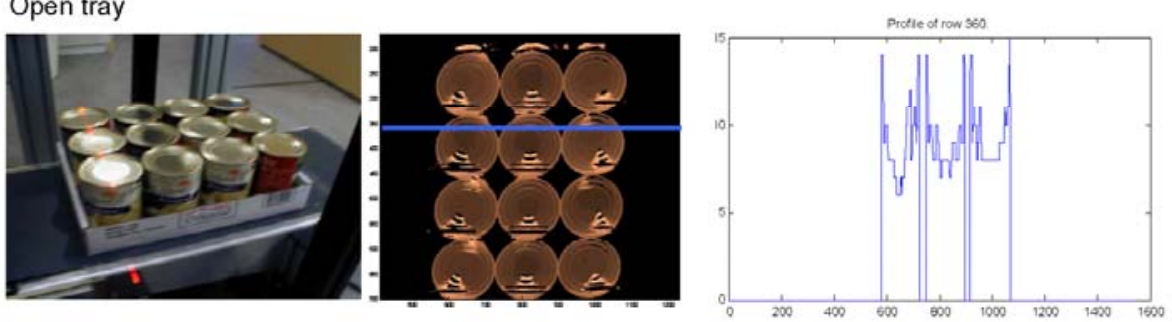


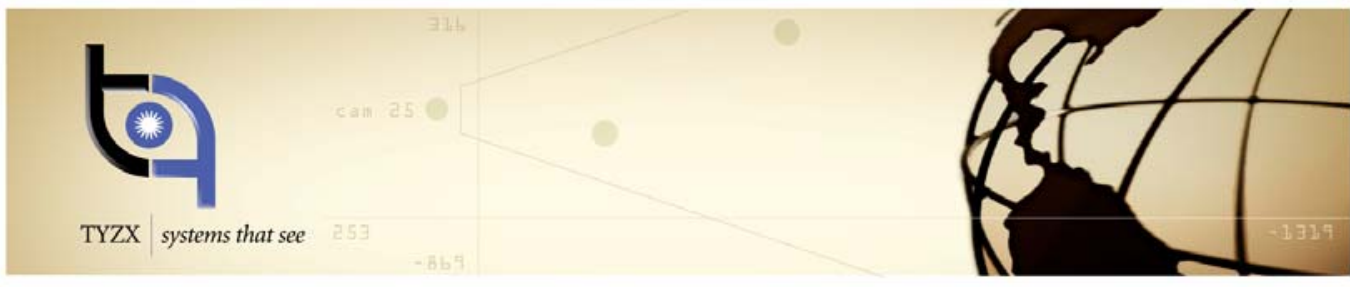

\title{
Company \& Technology Overview
}

\section{Gaile Gordon, VP of Advanced Development}

\author{
WWW.TYZX.COM
}

workshop slide \#205

\section{TYZX Overview}

$\rightarrow \rightarrow$ Moving the physical world online

- Systems that see, interpret and respond to the real world

- Real time Information and control

- Commodity 3D perception systems for smart products

$\rightarrow$ Applications

- Interactivity with users, real world environments

- Automation, mobile robot navigation and safety

- Vehicles, security systems, defense systems, entertainment

$\rightarrow$ Approach

- 3D passive optical sensing

Provides 3D data and registered 2D appearance image

- Common hardware/software platform for multiple applications

Cross market technology synergy

- Custom ASICs for performance and cost

- "3D Middleware" and development platforms to reduce OEM's TTM

workshop slide \#206 


\section{Stereo Vision}

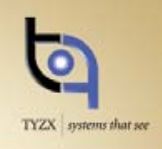

$\rightarrow$ Dense stereo vision

- Uses local texture to estimate depth for every pixel

- Expensive operations need custom hardware

.... Benefits

- Full frame of 3D data at high frame rates

- Operation in ambient light (passive)

- Works with a variety of sensors (IR, UV, color, ...)

- Flexible operating range through choice of baselines/lenses

$\rightarrow \cdot$ Use where

- Speed \& latency are important

- Environment is poorly constrained (natural scenes and objects)

\section{Markets}

$\rightarrow$ Automotive

- Pedestrian detection

- Lane keeping

- Occupant sensing

$\rightarrow$ (Person) Tracking \& counting

- Security and Surveillance

- Entertainment and Marketing

$\rightarrow$ Robots

- Autonomous systems Defense and consumer

- Vision guided vehicles

- Vision guided servoing

- Safety applications
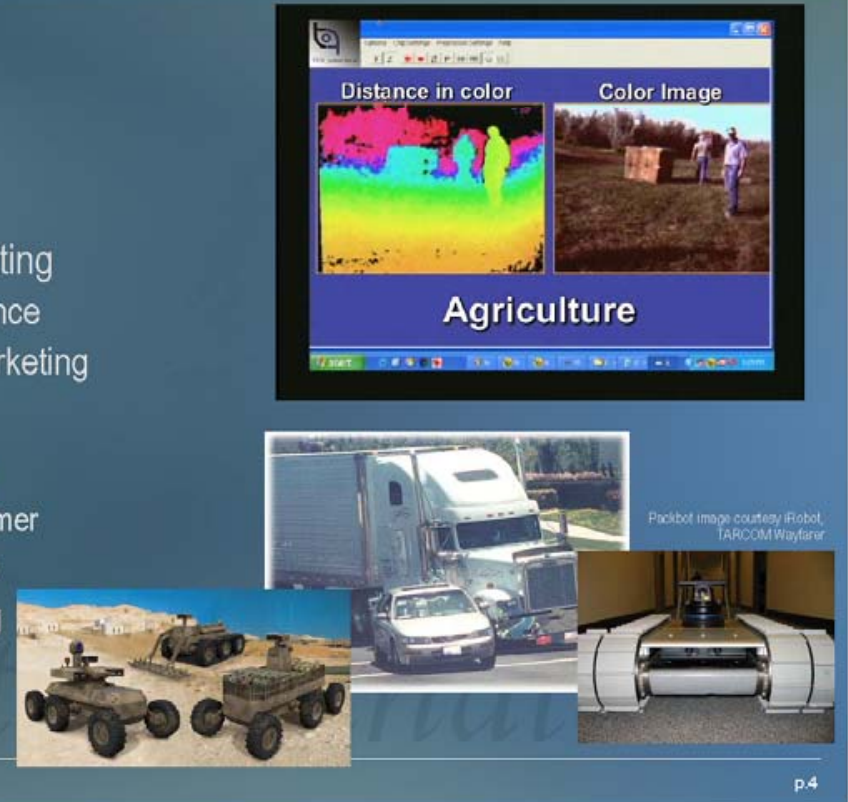


\section{Systems}

$\rightarrow$ Development Systems

- TYZX hardware hosted by PC

- Stereo camera

Configurations for different applications

- Software

Development libraries, Calibration software

Linux and Windows

Direct migration to G2 Vision System
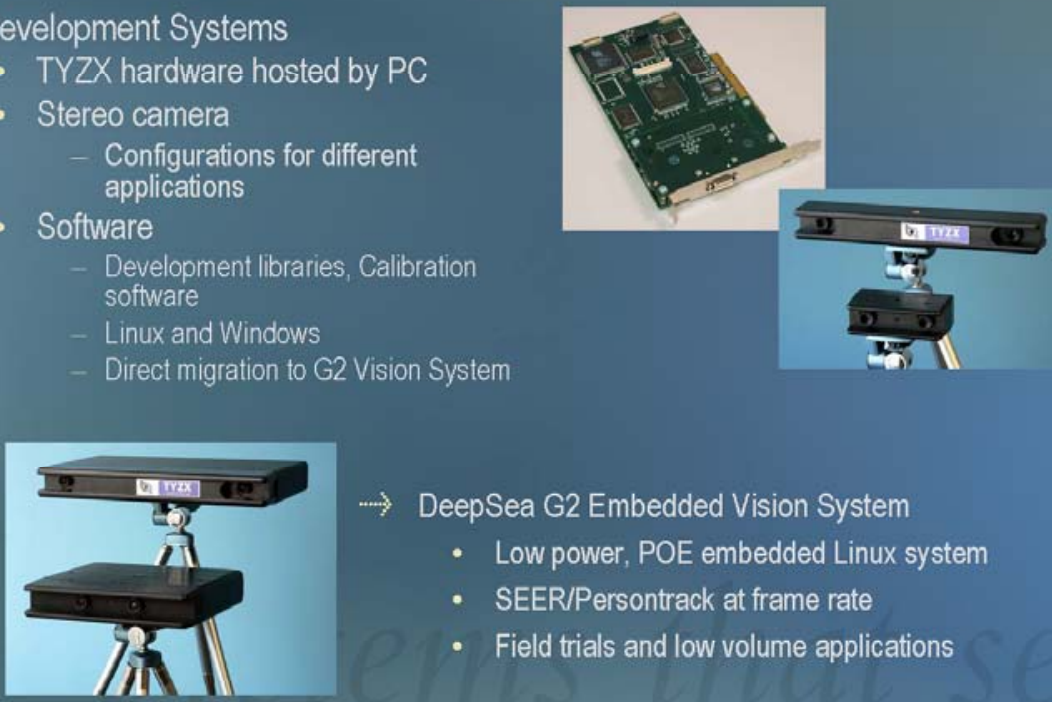

$\rightarrow$ DeepSea G2 Embedded Vision System

- Low power, POE embedded Linux system

- SEER/Persontrack at frame rate

- Field trials and low volume applications

\section{DeepSea G2 Hardware Processing}

.... High speed stereo is valuable, yet post-processing is still slow and expensive, so:

$\rightarrow \rightarrow$ Move more basic operations to fast, low cost hardware

- Hardware rectification

- Background modeling

- ProjectionSpace ${ }^{\mathrm{TM}}$

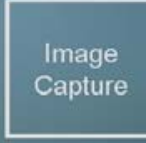

"on the CMOS

Imager curve"

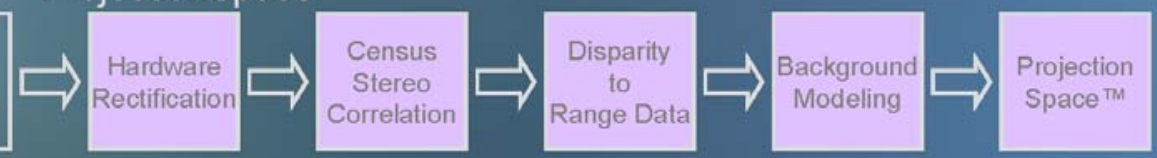

DeepSea G2 


\section{ProjectionSpace Interface}

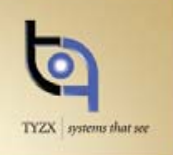

$\rightarrow$ Raw range data is row/column array of measurements (range "image")

$\rightarrow$ Convert to metric coordinates (xyz)

$\rightarrow 6$ DOF rotation to world coordinates

..$\rightarrow$ Quantize data into cells or "project" data onto a plane

$\rightarrow$ Also expensive and benefits from custom hardware

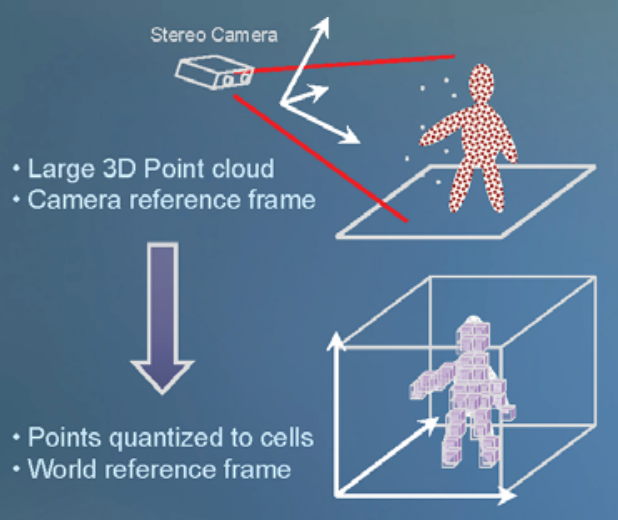

\section{Security \& Surveillance Example}
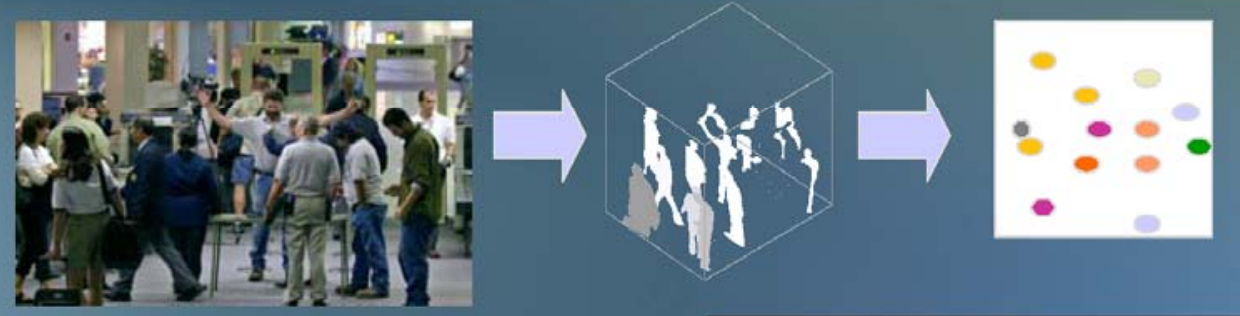

$\rightarrow 3 \mathrm{D}$ Vision excels at person tracking

- 3D Aids segmentation

- Color aids discrimination

$\rightarrow \rightarrow$ Places objects at a specific location

$\rightarrow$ Robust with respect to lighting changes

$\rightarrow$ Fast to provide direction and velocity vectors

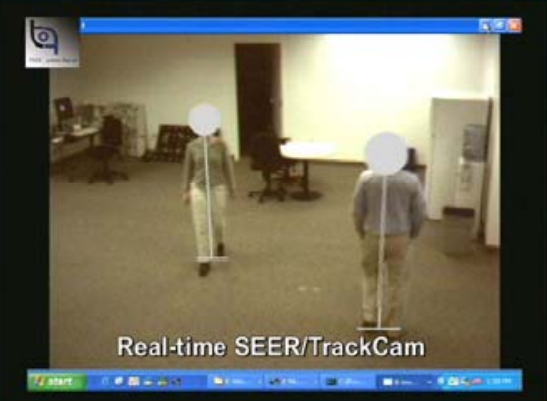




\section{Robust, Precision Stereo Cameras}

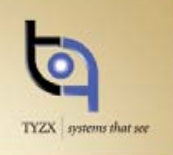

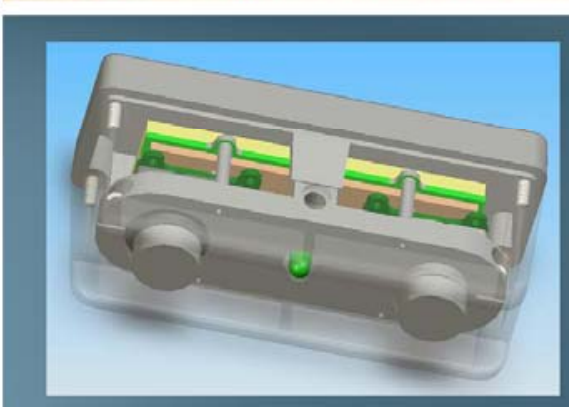

Standard Cameras

$\rightarrow 3 \mathrm{~cm}, 6 \mathrm{~cm}, 22 \mathrm{~cm}$ Baseline

$\rightarrow$ Kodak 9618 mono and color

$\rightarrow$ Micron MV022 mono

- Wide VGA format

- Full frame shutter

- HDR

- Color

- 60 fps

$\rightarrow$ Robust construction

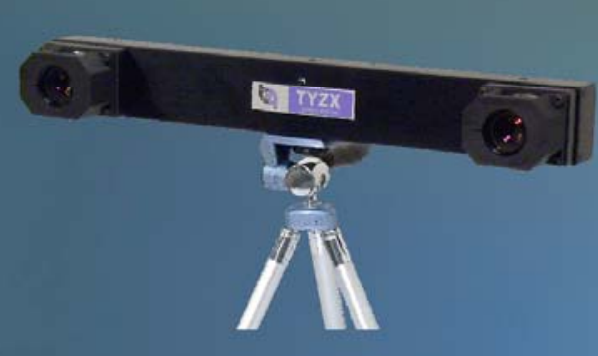

Custom Cameras

$\rightarrow 33 \mathrm{~cm}$ baseline

- Custom baselines available

$\rightarrow \rightarrow$ Rugged

- Stiff, aluminum construction

- C-mount with "Lens Cage"

$\rightarrow \rightarrow$ Exposure control

- Unified motor iris, integration time \& gain

- HW assist

\section{Quick Survey}

$\rightarrow$ Stereo vs. 2D video

- More reliable and easier segmentation and application processing

$\cdots$ Dense stereo vs. Feature-based and multi-camera

- Better for capturing unplanned events

- Imager pair maintains calibration/registration

- Less suited for photogrammetry, though std triangulation can still be performed

..$\rightarrow$ Stereo vs. range sensors (radar, lidar, sonar)

- Passive

- Higher resolution

- Faster, less noisy

- Wider FOV, flexibility with respect to operating distances

- Stereo vision provides "fused" appearance image for free

$\rightarrow$ One technology - many sensors

- Just change stereo baseline \& lens

- Millimeters to 100 meters 


\section{Deployment in the Real World}

$\rightarrow$ Four DeepSea G2s tracking people

$\rightarrow$ Two exterior glass walls

$\rightarrow$ Thousands of Watts of dynamically controlled flashing LEDS
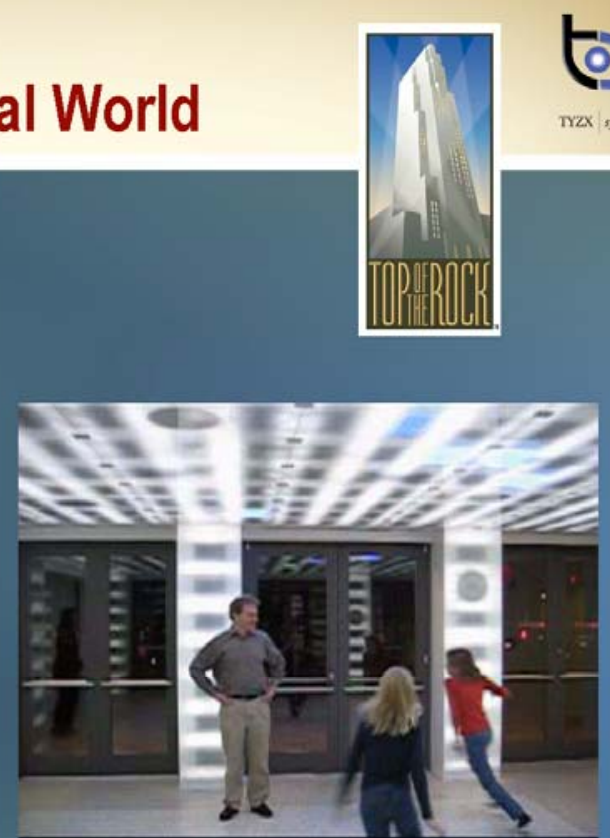


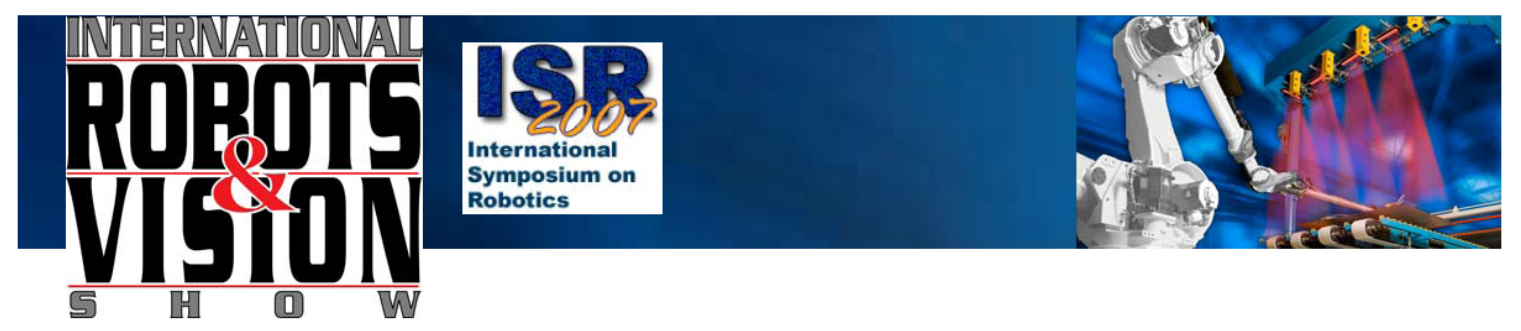

\section{How to Implement Bin Picking in your Manufacturing Operation \\ Adil Shafi \\ President \\ SHAFI, Inc.}




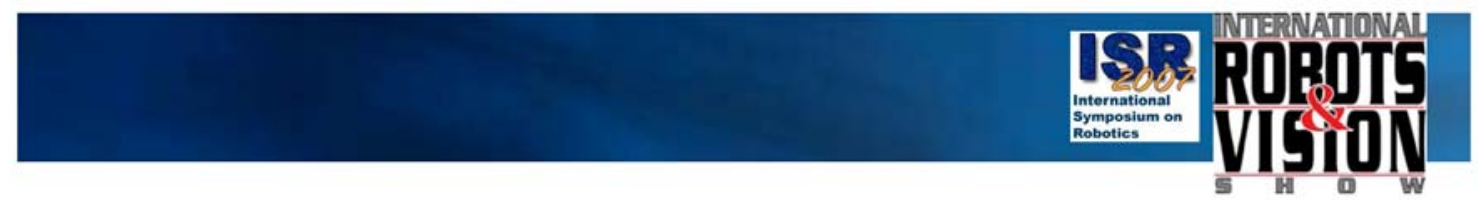

\begin{abstract}
This article is targeted towards the End - User manufacturing community. It is intended to provide a brief overview of Bin Picking's progress towards reliable and widespread use, with Vision and/or Light Guided Robotic techniques, and then to provide a methodology to consider, carefully test, and implement reliable Bin Picking.
\end{abstract}

\title{
Turning the Completely Impossible into the Obviously Possible
}

When Thomas Alva Edison began to work on inventing a light bulb, it was generally considered an unreliable and impossible task. When with self belief and perseverance he succeeded, he looked back and said that he had to succeed since he ran out of methods that could not succeed. Today, satellite images show impressive images of lights in industrialized regions on earth at night.

Learning from failures and the experience of others before him, Sir Edmund Hillary defied the conventional reservations of his time and summitted Mount Everest. Today, so many people summit Mount Everest each year that it is commonly joked that soon we will have a weather insulated escalator to go up to the top.

In our manufacturing community we have similar parallels. A generation ago, most welding was done by people, often with inspectors after welding stations. Today, manual welding is questioned and rare. Just six years ago, 3D Vision Guided Robotics performing AutoRacking (or pick or place stamped metal parts from or onto racks) was virtually unprecedented. Presently, we have hundreds of cells running AutoRacking reliably in our industry and some companies implement AutoRacking on every new manufacturing program.

I believe that the same is true of Bin Picking. A few solutions have been running in production for more than three years and more are being implemented each year. Within a decade or so, all Bin Picking will be automated. Our next generation will wonder why people would want to pick parts manually, more slowly and more expensively than a fast robot from a bin. Manual bin picking will then become questioned and rare.

\section{The Enablers}

Bin Picking, in the past three years, has quietly but steadily made advances in commercial production lines. A good review of successful solutions in our manufacturing industry was published in Automation World's February 2006 issue, unw.automationworld.com. The article was entitled "Vision Guided Robotics: In Search of the Holy Grail".

Page 2

workshop slide \#217 


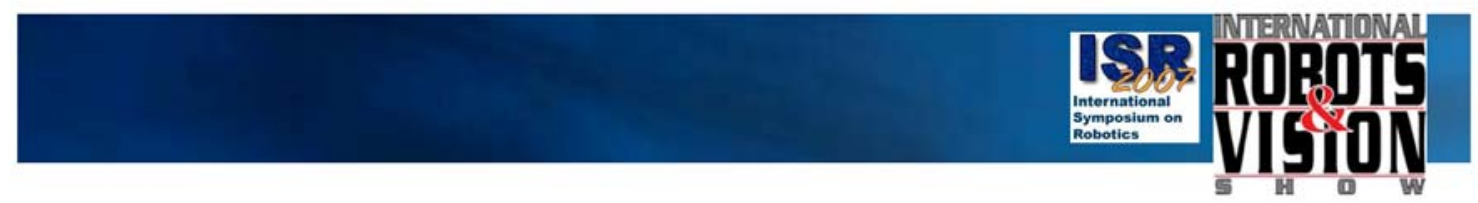

Ease of Bin Picking is driven and prioritized by two factors: 1) The geometry of the part, and 2) The degree or severity of randomness of parts in bins. The first, easiest, and financially most justifiable solutions have been in the automotive powertrain area; most notably engine blocks. These parts are well machined, are rich in geometric features, skewed slightly in $x, y, z$, yaw, pitch, roll directions and are heavy (thereby slow and hence expensive to manually handle). This has been a perfect first storm to enable Bin Picking.

There are many enablers currently driving more solutions into the fold of reliable Bin Picking. These include: Advances in computational processing power, vision recognition tools, mathematical algorithms,

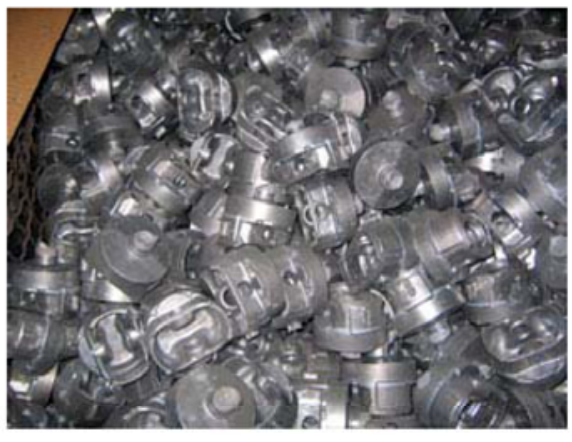
flexible lighting, a continuous reduction in commercial pricing, and a growing collection of techniques in handling, gripping and staging an overall problem into more easily handled steps.

A rough analogy is that $16=4 \times 4$, but 16 is also $4+4+4+4$. Addition is easier to do than multiplication. The same problem can be reduced into several smaller equivalent problems.

A tough bin picking challenge can be simplified by breaking the problem into individual retrieval only first, which may be imprecise in finding a part centroid, but then using a simpler 2D pick and place stage for precise final placement. Such two-stage operations can reliably run entire bins and meet a six second part-to-part, bin acquisition to precision pins placement cycle time. Fast, fixed mount camera solutions are now running in production at four second part-to-part cycle times.

\section{Good Applications That Are Ready for Reliable Bin Picking in Production Now}

The following applications have now become feasible for reliable Bin Picking:

\section{Automotive}

- PowerTrain (Engines, Cylinder Heads, Axle Shafts, Differential Carriers, Pinions, Round Parts with Stems, Connector Rods, Piston Heads, Brake Rotors and Stacks of Gears).

- Stamping (Flat or bent metal plates with multiple holes, roughly stacked stampings with a progressive skew).

- Final Assembly Products in Boxes in T/C/F (Trim Chassis Final) pick operations for placement into cars on moving lines; see related discussion about Vision Servoing at the Robotic Industries Association website http://mww.roboticsonline.com/ 


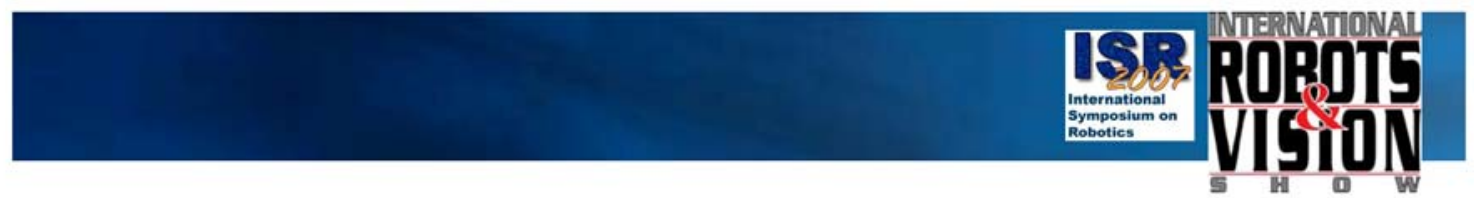

\section{Packaging}

Strips of medical tablets, flat but randomly arranged in boxes.

Bags of products e.g., chips, salsa, cheese, cement, etc.

Lateral or upright layers of tubes (copper, plastic, PVC).

Layers of products e.g., wooden planks, plastic sheets.

\section{How to Implement Bin Picking in your Manufacturing Operation}

The following steps are recommended to evaluate, justify and implement Bin Picking.

The instructions below are a bit precise but not difficult to follow.

1. Take pictures of your parts with a cell phone or a digital camera from an electronics store. You will need two cameras for your part and bin image analysis.

Individual Part Pictures (IPP)

2. Consider each part that you manufacture. Place each of your parts on a flat surface. Review the multiple stable resting positions in which each part can be placed on a flat surface (for example, a soft drink can has two stable resting positions: One "standing up" with its circular footprint on the flat surface, and one "lying on its side" with its circular planar ends perpendicular to the flat surface (the resting position in which it can roll on a flat surface).

3. Then for each of your parts, take a picture of each Stable Resting Position (SRP). The camera should be aimed at about a 45 degree angle to the flat surface, looking down towards the part. Collect this as your library of Individual Part Pictures (IPP). This is essentially a two-dimensional array of pictures, where the first index is your part number, and the second index is the part's SRP.

\section{Bin Randomness Pictures (BRP)}

4. The next step is to take each of your part types, and review how randomly they are found in actual bins in your manufacturing operation.

5. Using a tripod or a temporary structure, setup two fixed-mount cameras above each bin. Depending on the size of your bin, adjust the size of the view so that the Field of View (FOV) of your image is indeed the entire bin. Place the first camera directly above the bin pointing straight down or perpendicular to the flat horizontal plane of the bin below. Let's call this Camera 1 or C1. Place the second camera at a 45 degree angle above the bin, looking downward, so that it sees the C1 scene from an angle from any side (select one fixed side) of the bin. Again setup the FOV so that it has as much part content in it as possible as what $\mathrm{C} 1$ can also see. Let's call this camera at 45 degrees Camera 2 or $\mathrm{C} 2$. When looking at a bin, the planar 2D views of $\mathrm{C} 1$ and $\mathrm{C} 2$ will not be in the same direction nor scale and the $\mathrm{C} 2$ images will be skewed and that is fine.

Page 4

workshop slide \#219 


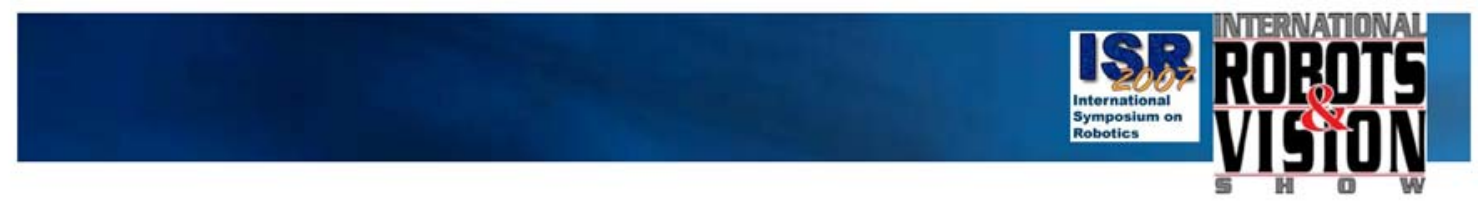

6. Then for each of your parts, place a bin of parts below C1 and C2 (as they normally occur in production to the level or randomness that you typically find them). Take multiple pictures of each bin and several examples of randomness of parts that you will see. Organize and maintain a pair of $C 1$ and $C 2$ image pairs for every scene.

Take This Pictorial Information to the Experts: Evaluate and Believe by Seeing Demos

1. Take this pictorial information to experts in the field of Bin Picking. You can use an Internet search engine (enter "Bin Picking"). Request examples of their past work as well.

You can also attend and meet speakers at the 3D Bin Picking Conference track at the International Robots \& Vision Show in Rosemont, Illinois (Chicago) on June 12 14, $2007 \mathrm{http}: / / \mathrm{www}$.robots-vision-show.info/robots_vision_show_info.html. There will be several Bin Picking demonstrations running at the show.

2. Request an evaluation of your parts from the pictorial information collected above. It is then possible to obtain a budgetary estimate to automate your Bin Picking operation. If the payback on investment is justifiable, then proceed with the following steps.

3. The first key to success is to insist on a pre - sale demonstration with exactly your parts. This is a critical step to not misunderstand and to not create failures. It is very important to ask for a completely reliable, uninterrupted retrieval of all parts, or negotiated manual intervention for certain cases of part randomness. It is the only way to adequately protect the risk in these projects for five parties : End - User, Systems Integrator, robot company, vision company, and software enabling company.

Sometimes these roles are provided by the same company, however Bin Picking experience and a single line of project responsibility from a Systems Integrator is critical to your success in this area.

\section{Seeing is Believing}

It is highly recommended that your factory floor personnel visit and review vendor demonstrations, since they often know of rare and exceptional cases that can stop production. It is critical to gain a comfort level by seeing several, continuous, uninterrupted and realistic demos running from completely full to completely empty bins before issuing a purchase order.

\section{Part Variation Management}

4. The second key to success is Part Variation Management (PVM) in your operations. It is very important to separately study, log, plan and manage manual-to-automatic

Page 5

workshop slide \#220 


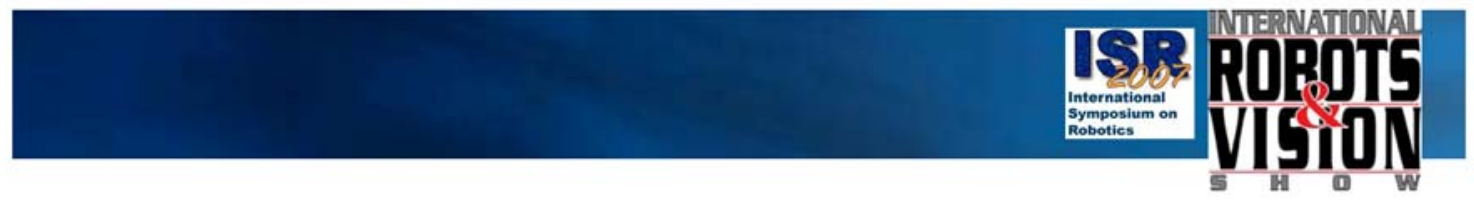

retrofits versus new part programs. In a retrofit situation, it is possible and recommended to take hundreds of unobtrusive pictures (see $\mathrm{C} 1$ and $\mathrm{C} 2$ image gathering methods above), and to be able to run simulated pickups of those images offline.

This process protects being caught off guard after good laboratory demos and runoffs at the vendor site, while remaining unaware of true variation in a plant. Sadly, this is often realized late in a project when the vendor arrives at the End - User plant for final implementation, only to discover that a number of variation cases were unexpected, misunderstood and unplanned for in advance.

These types of mistakes create disillusionment and delay in future confidence, and ultimately delay the time advantage in financial benefit to End - Users. It often takes a year or two for a typical End - User to recover, reinvestigate and reinvest. In the meantime, other global End - Users gain competitive advantage by avoiding these mistakes

5. Thirdly, it is recommended that you review and benchmark, through actual test, ease of use for non - technical operators, training at Operator, Technician and Engineering levels, a FMEA (Failure Mode Engineering Analysis), and rigorous procedures for backups, version control, and access to $24 / 7$ vendor support.

\section{Conclusion}

Bin Picking is a manufacturing solution whose time has now come. There are many examples of Bin Picking that are ripe for success and financial benefit to End - Users. The content above provides a methodology for analysis and evaluation. It also provides project management guidelines critical to protect End - User success.

Page 6 
The History and the State of the Art of Laser Tracker Technology

\section{and Applications}

Kam Lau, Ph.D.

\section{President}

Automated Precision, Inc.

Maryland USA

Metromeet 2008

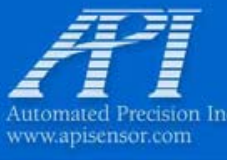

workshop ज्ञात 2222

\section{Topics of Discussion}

- Brief introduction

- History of Laser Tracker Development

- Theory of Laser Tracking and comparisons of different tracking techniques

- Evolutions of laser tracker designs

- Tracker Traceability (ASME B89.4.19 Vs USMN)

- Advances in tracker applications and accessories

- Summary of Discussion

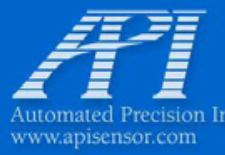


- Ph.D. in ME, U of Wisconsin -Madison 1982

- Worked for NIST (formerly NBS) between 82-87

- Founded Automated Precision, Inc. in 1987

- Major metrology system inventions:

- 3-/6-D Laser Tracker (Co-inventor Dr. Robert Hocken)

I-probe, Iscan, 6-D Smart Track, RTOF ADM

- RapidScan- a 3-D optical surface scanner

- 3-D locking high-precision CMM probe

Deep Bore Profiler for measuring profile of deep holes XD Laser Interferometer System for simultaneous multisaxis $\mathrm{CMM} / \mathrm{machine}$ tool error measurements

1 CNC Machine G/T Error Model and Compensation

\section{API Headquarter at Rockville, MD USA}

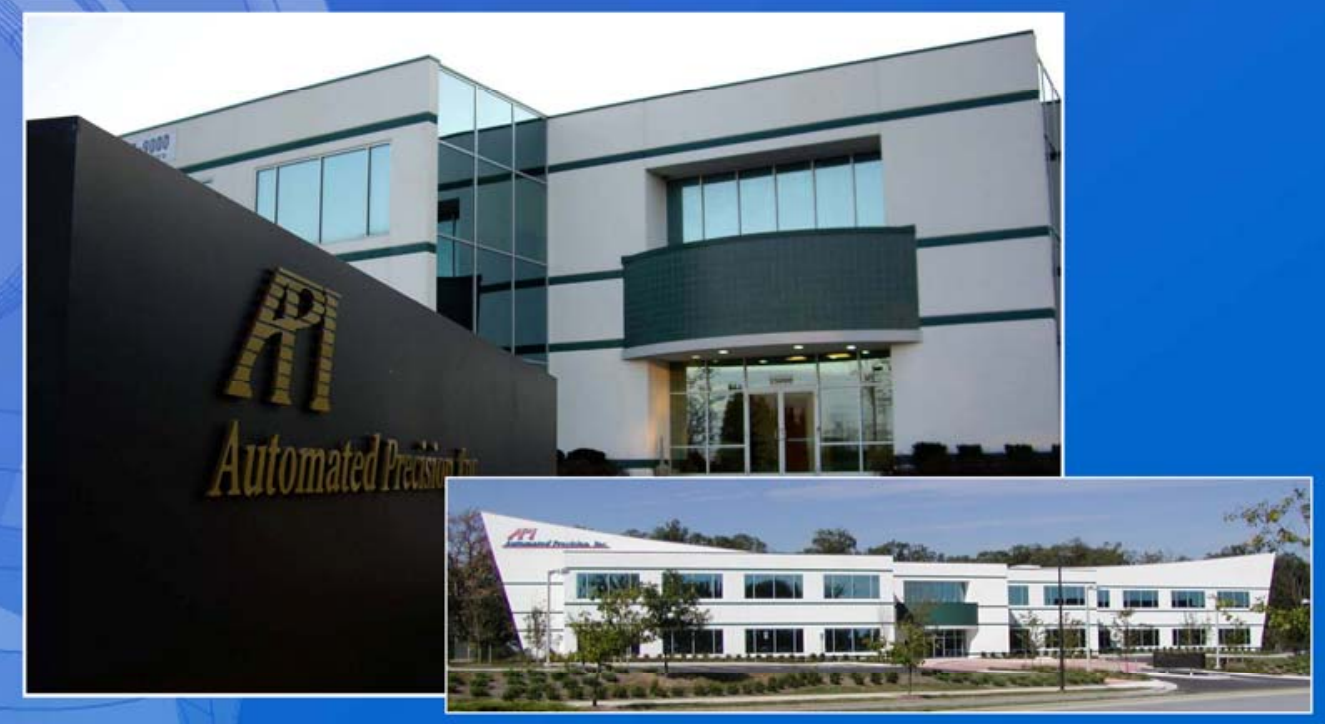

ISO 17025 metrology lab accreditation 46,000 sq. ft., temperature controlled environment $45^{\circ}$ by $90^{\circ}$ vibration isolated laboratory

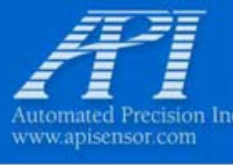




\section{History of Laser Tracker}

1979-80, Itek first demonstrated a 4-head

trilateration laser tracking system for large

optical surface measurement (Itek patented)

- In 1982, the single-beam laser tracker was

conceived and demonstrated. NIST eventually

patented a 3/5-D laser tracker (Kam Lau and

Bob Hocken as co-inventors)

- In 1983-85, Chesapeake Laser (CLS) adopted the Itek concept and built a prototype with funding from the US Navy

- $\quad 1988 / 89$, Boeing successfully conducted a full test and evaluation of the first API 3-D singlebeam laser tracker

- 1989, first commercial SB laser trackers introduced by API/Leica, CLS followed

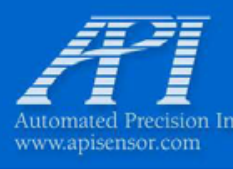

\section{History Cont'd}

- 1991 SMX acquired CLS

- 1994/5 Leica Tracker combined IFM+ADM capability, followed by SMX within a year

- 1999 API entered the market with the $2^{\text {nd }}$

Generation Laser Tracker T2 (on-shaft mounting laser)

- 2002 API introduced T2+ with IFM+ADM capability, Faro acquired SMX

- 2004/5 Faro introduced X Series (fiber-guided laser)

- 2005 APYintroduced T3 and OT with Turbo ADM

- 2005/6 Leica and API introduced handheld probes

- 2008 Leica introduced Absolute Tracker

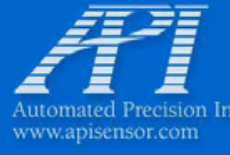




\section{Revolution of an industry}

Since we adopted the use of laser tracker, we estimate a corporate saving of 4.5 billion dollars..." quote from a senior manager at a major aircraft manufacturer in year 2000

The ease of use, accuracy and costeffectiveness of laser tracker have totally changed the ways how aircrafts are built. Other industries also experience the same magnitude of economic and productivity impacts. After 20 years of its introduction, the impact still continues ...

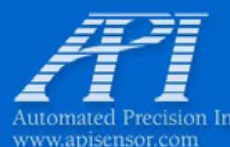

wrochop ज्ञात 2228

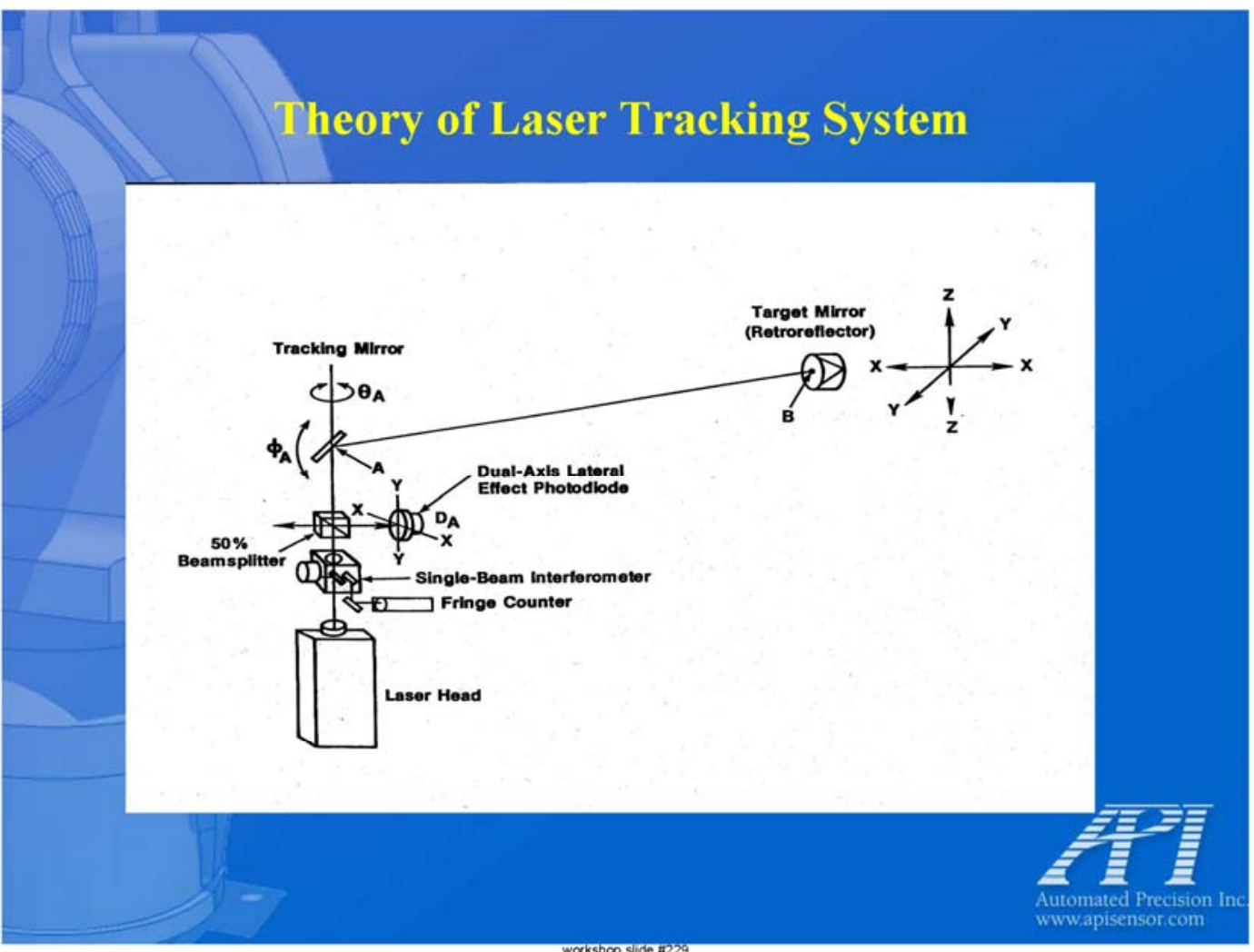



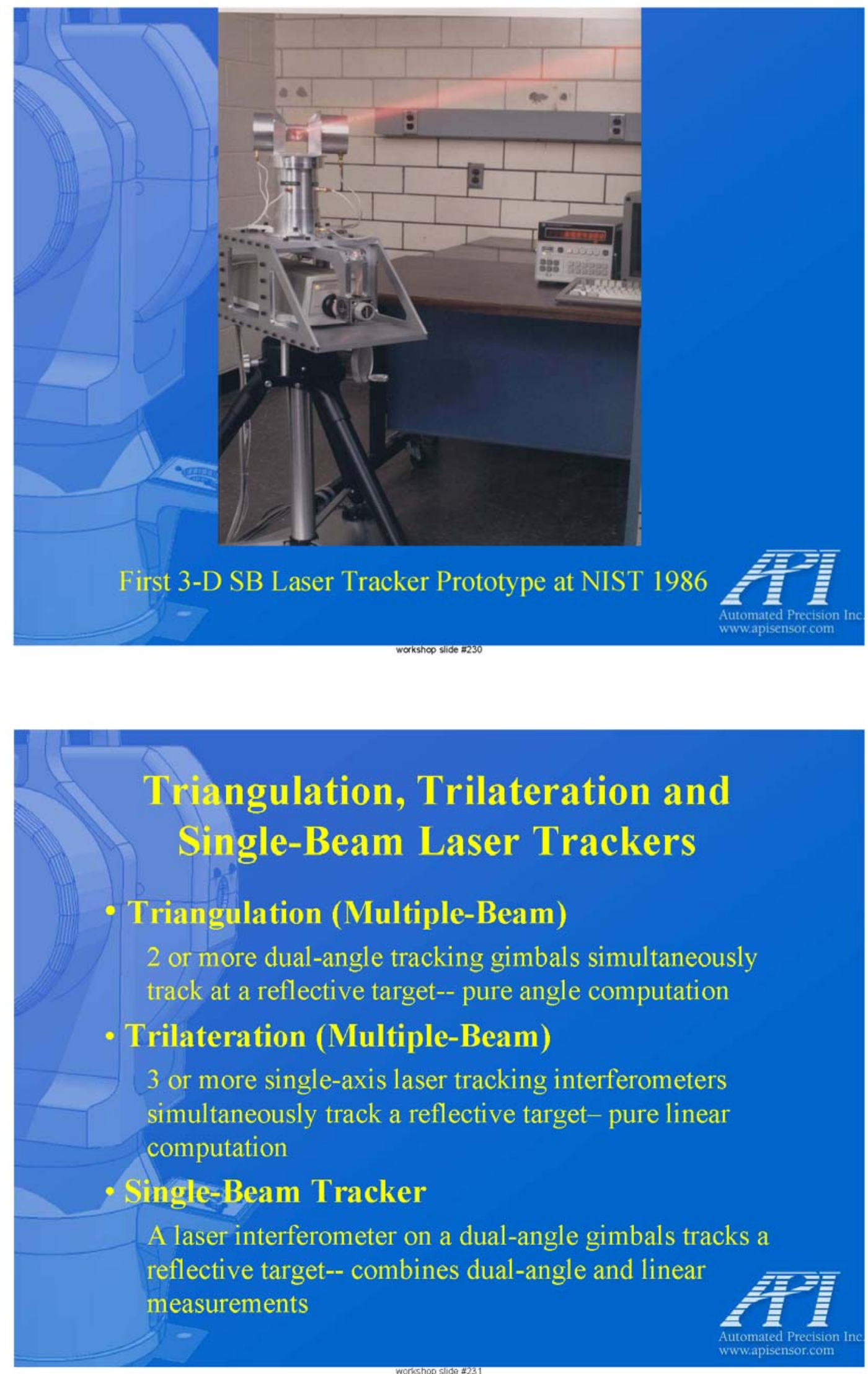


\section{4-Head Multiple-Beam Tracking System}

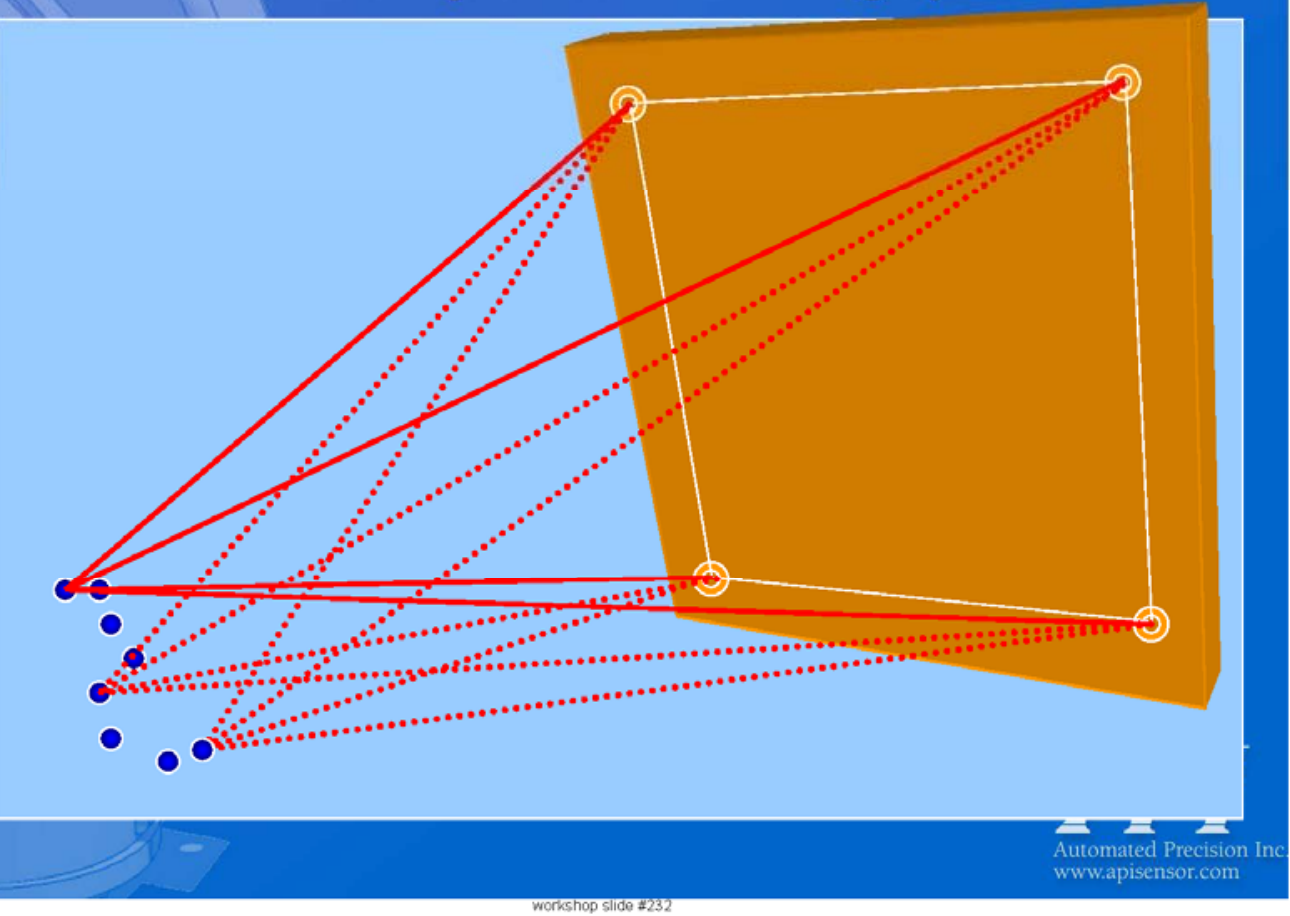

\section{Triangulation, Trilateration Vs}

Single-Beam Laser Trackers

Which is more accurate?

Primary Argument: IFM is more accurate than angular encoder

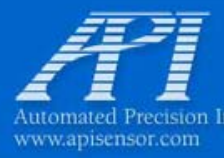




\section{Assumptions for Discussion}

- No environmental effects

- No consideration of metrology frame inaccuracy or instability

- No consideration of artifacts or setup errors

- Angutar uncertainty- 1 arc-second

- Linear uncertainty- 1 ppm

Measurement Uncertainty in

a Triangulation System

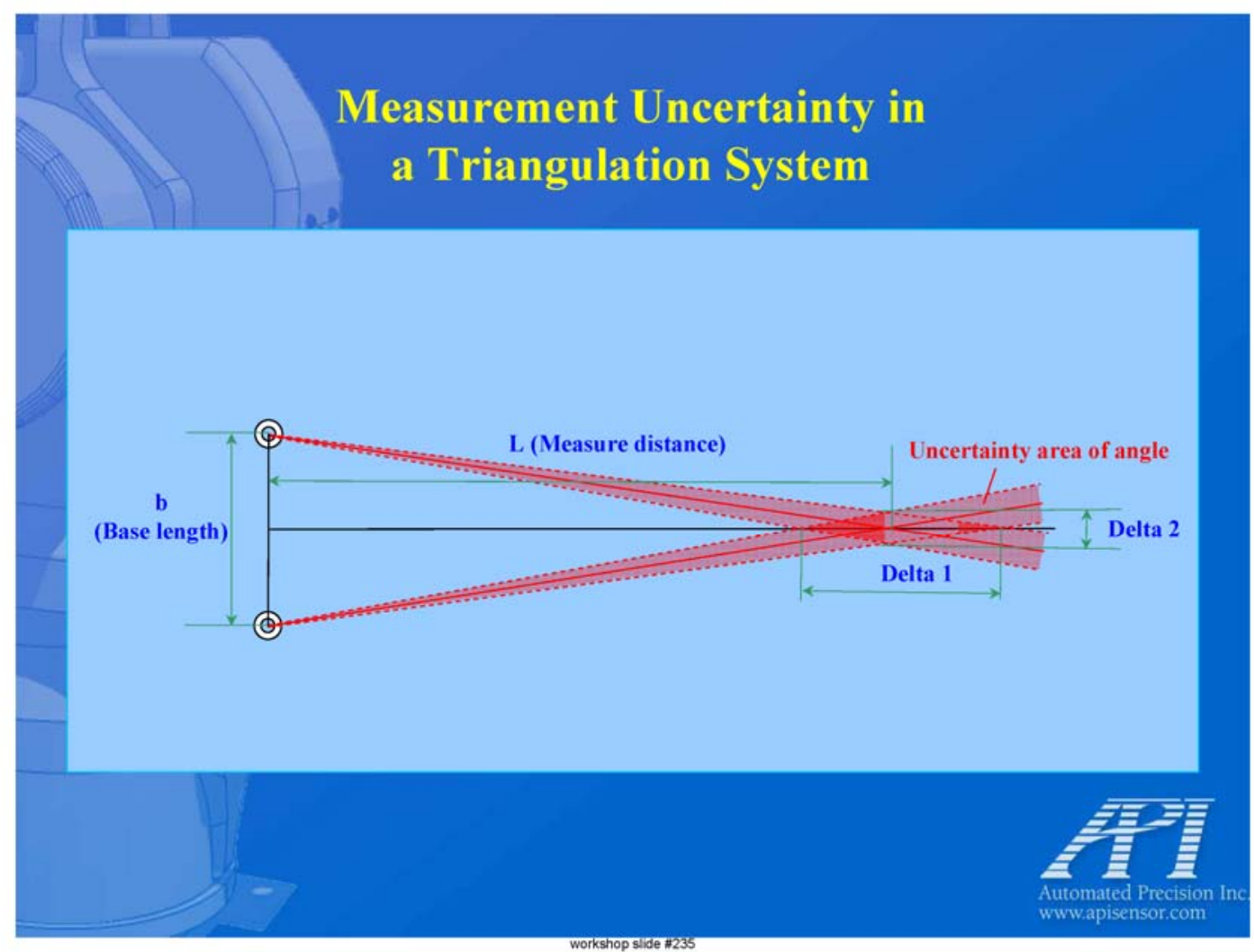




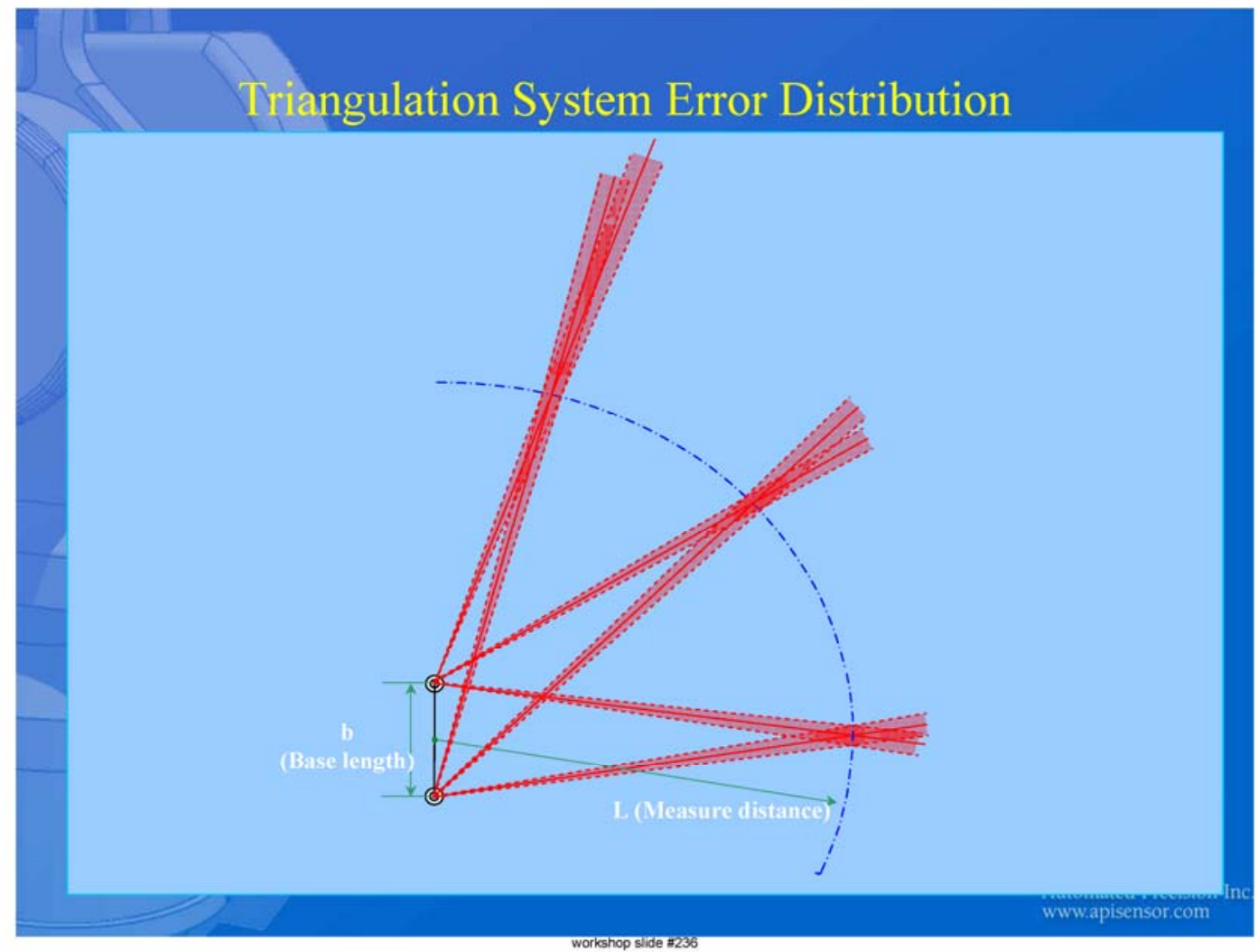

Workshop side

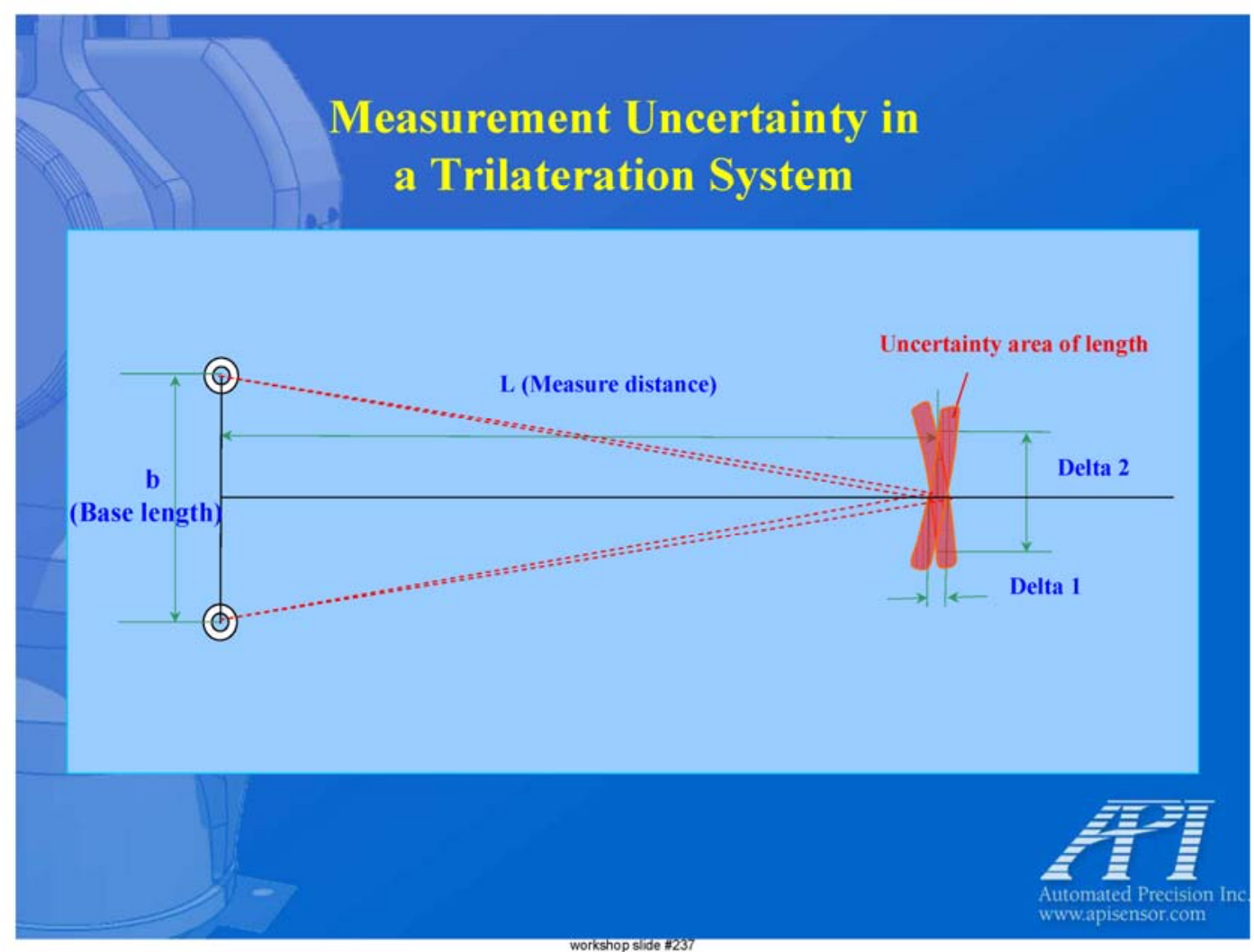




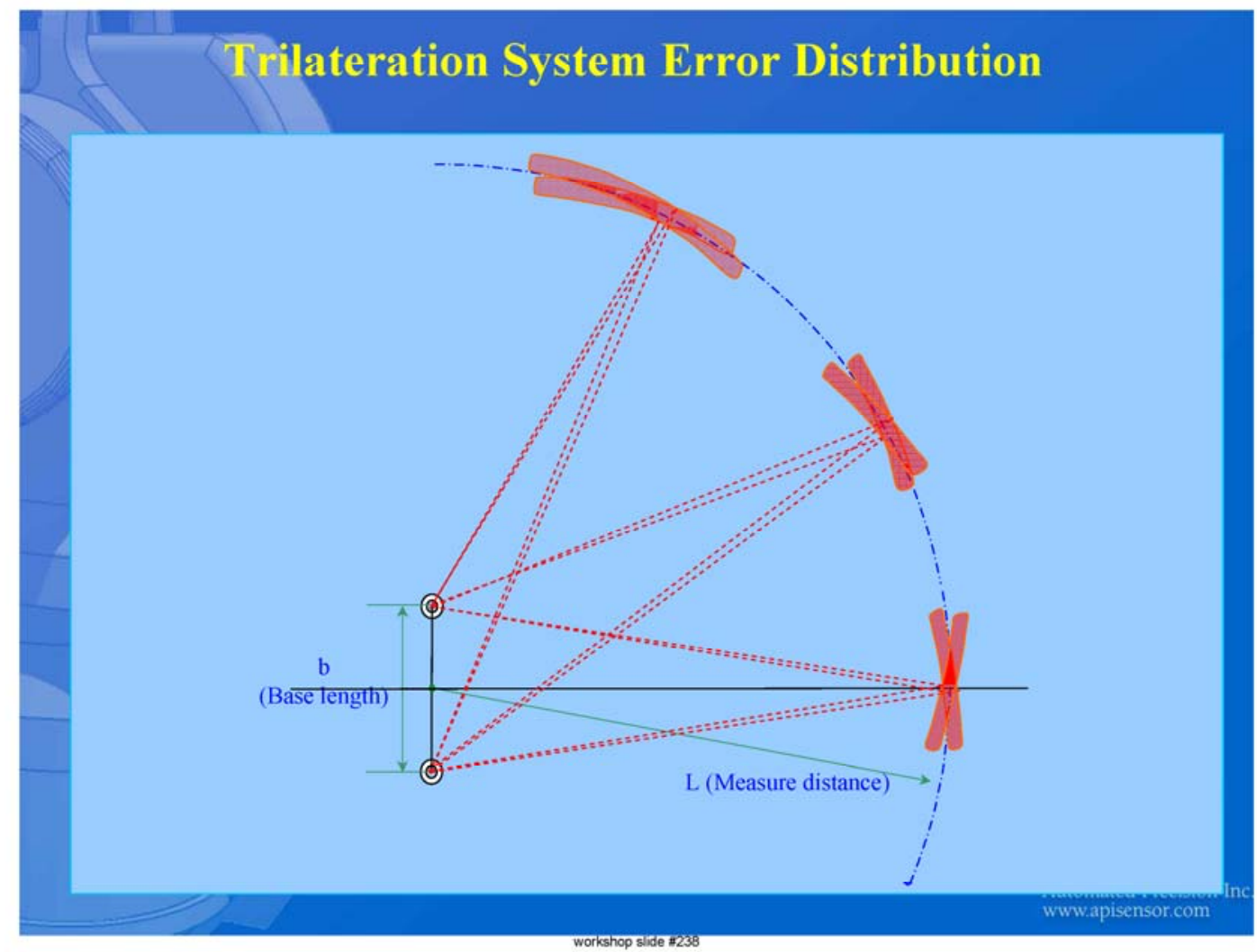

\section{Measurement uncertainty in Single-Beam system}

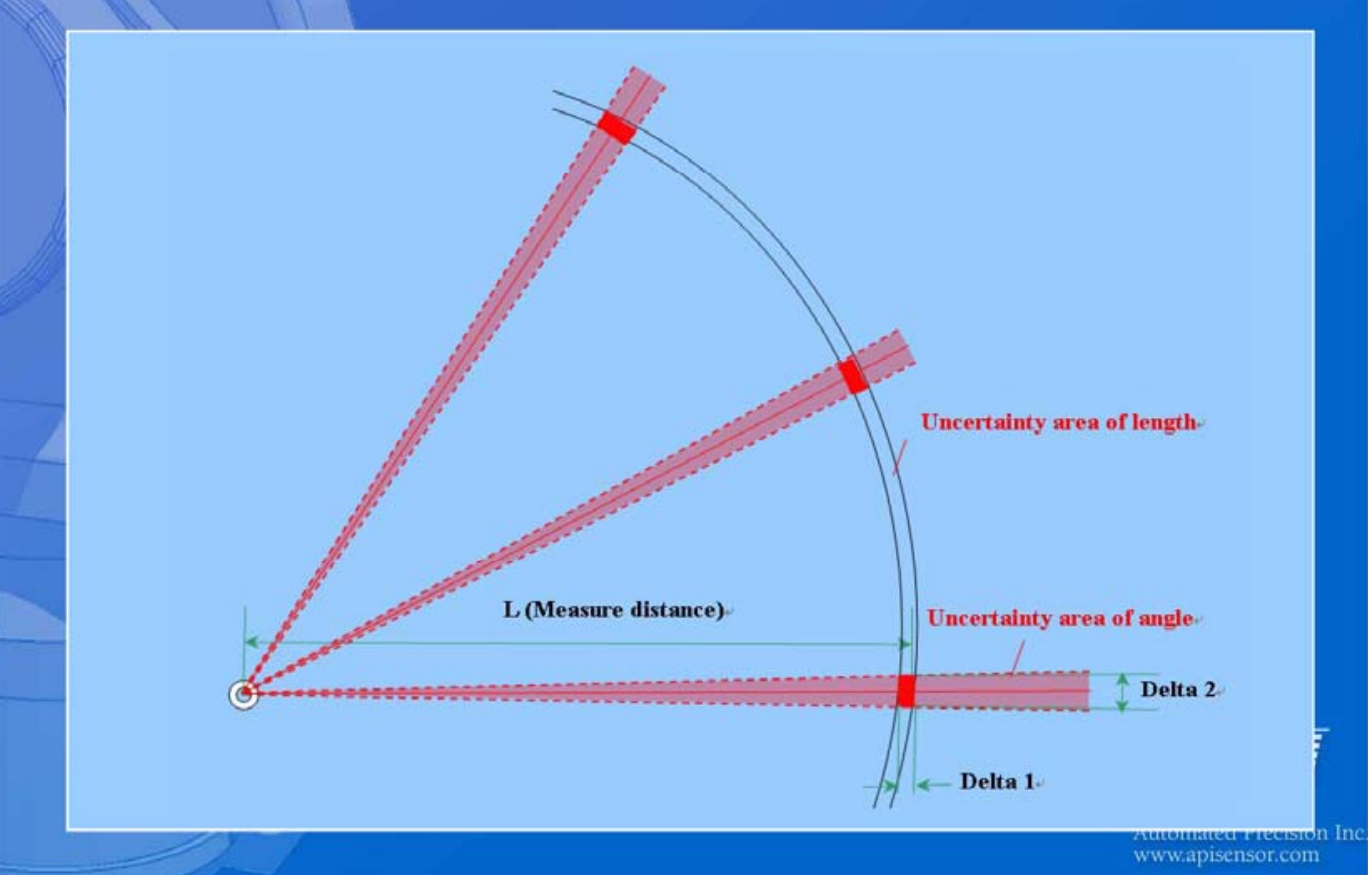




\section{Theoretical accuracy comparison of SB and MB}

tracking system

\begin{tabular}{|c|c|c|c|}
\hline $\begin{array}{c}\text { Measure } \\
\text { Distance } \\
(\mathbf{m})\end{array}$ & $\begin{array}{c}\text { Triangulation system } \\
\mathbf{b}=\mathbf{5 m} \\
\text { Delta 1 }(\mathbf{m m})\end{array}$ & $\begin{array}{c}\text { Trilateration system } \\
\mathbf{b}=\mathbf{3 m} \\
\text { Delta } \mathbf{2}(\mathbf{m m})\end{array}$ & $\begin{array}{c}\text { Single-Beam system } \\
\text { Delta 2 (mm) }\end{array}$ \\
\hline 1.5 & 0.0330 & 0.0030 & 0.0145 \\
\hline 2 & 0.0398 & 0.0042 & 0.0194 \\
\hline 2.5 & 0.0485 & 0.0057 & 0.0242 \\
\hline 3 & 0.0591 & 0.0075 & 0.0291 \\
\hline 3.5 & 0.0718 & 0.0097 & 0.0339 \\
\hline 4 & 0.0863 & 0.0122 & 0.0388 \\
\hline 4.5 & 0.1028 & 0.0150 & 0.0436 \\
\hline 5 & 0.1212 & 0.0182 & 0.0485 \\
\hline 10 & 0.4121 & 0.0682 & 0.0970 \\
\hline 20 & 1.5756 & 0.2682 & 0.1939 \\
\hline 30 & 3.5148 & 0.6015 & 0.2909 \\
\hline 40 & 6.2296 & 1.0682 & 0.3879 \\
\hline
\end{tabular}

\section{Theoretical Accuracy Comparison of SB and MB} Tracking Systems

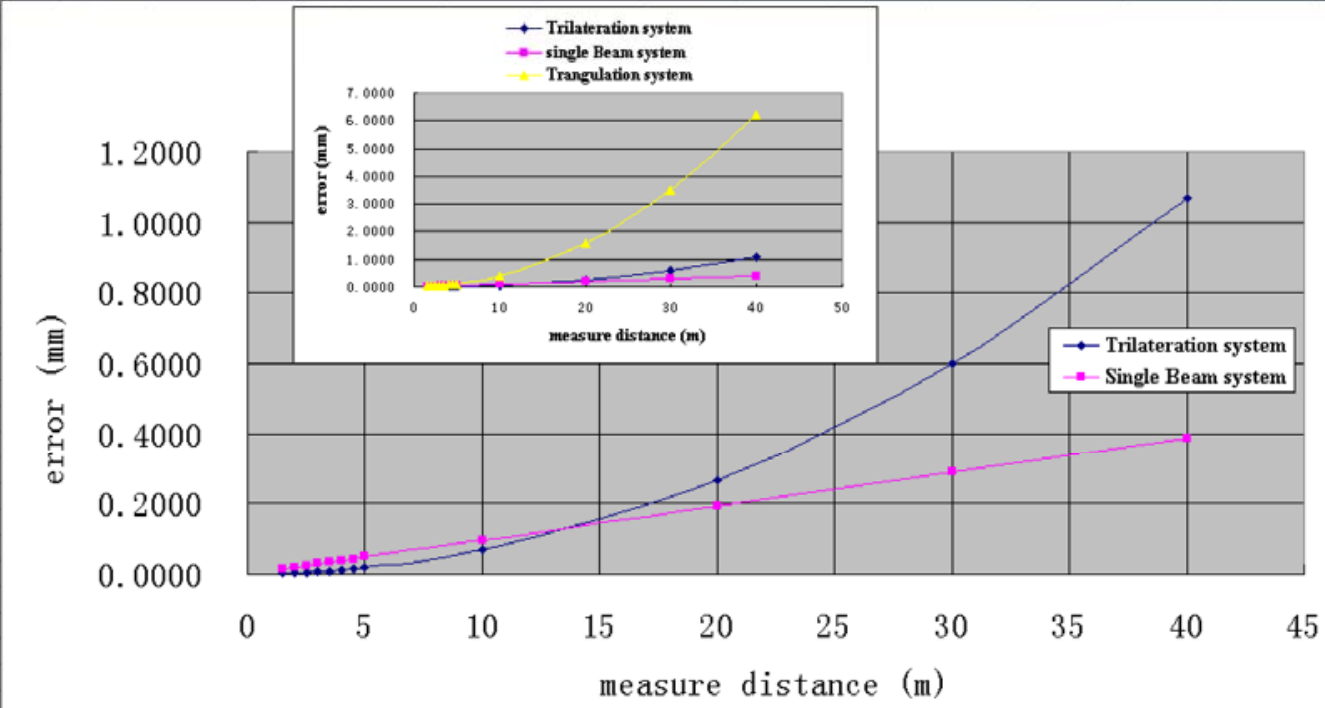




\section{Pros and Cons on SB Vs MB for Large-Scale Metrology}

- MB dominated by non-linear regions, optimum accuracy at $60^{\circ}$ envelop; SB is more linear

- $\mathrm{MB}$ requires artifact calibration to define base distances therefore reducing accuracy, SB does not

- Uncertainties in MBs crossing apex of SMR, metrology base frame stability, etc. compromise overall accuracy.

- Portability, cost, ease of use and accuracy is field certifiable make SB tracker more favorable for industrial applications

\section{Evolutions of Tracker Head Designs and Heat Management}

- Remote Vs On-shaft laser mounted

- Key principles to better head design

Axis symmetry for thermal stability

Shortest optical deadpath, minimum moving mirrors

Abbe' Principle compliance

Structural rigidity but no mass

- Heat Management

Remove (impossible) or minimize heat source

Incorporate heat source into the design

Workshop silde $\$ 243$

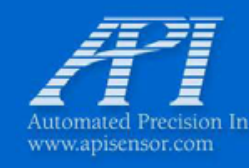



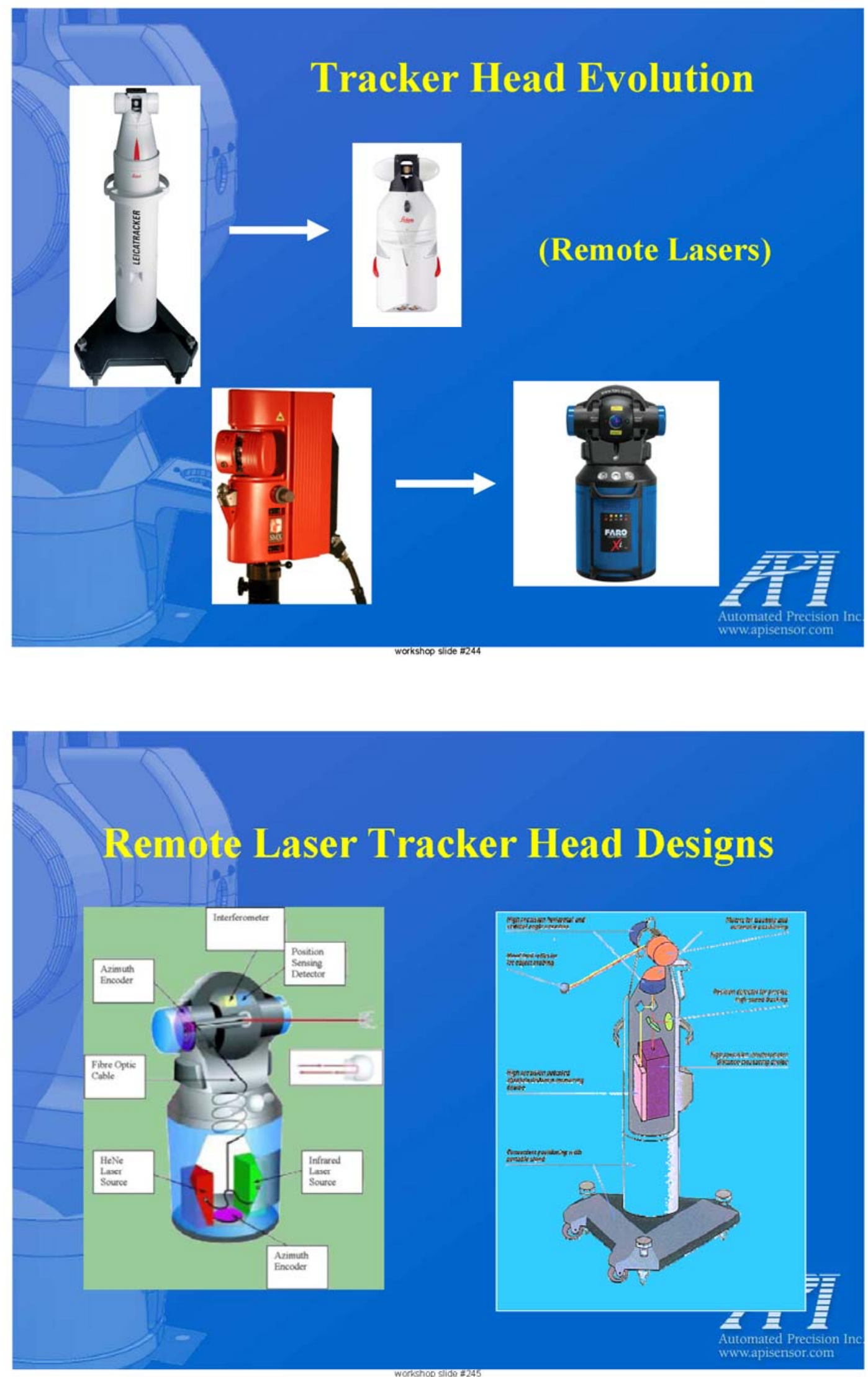

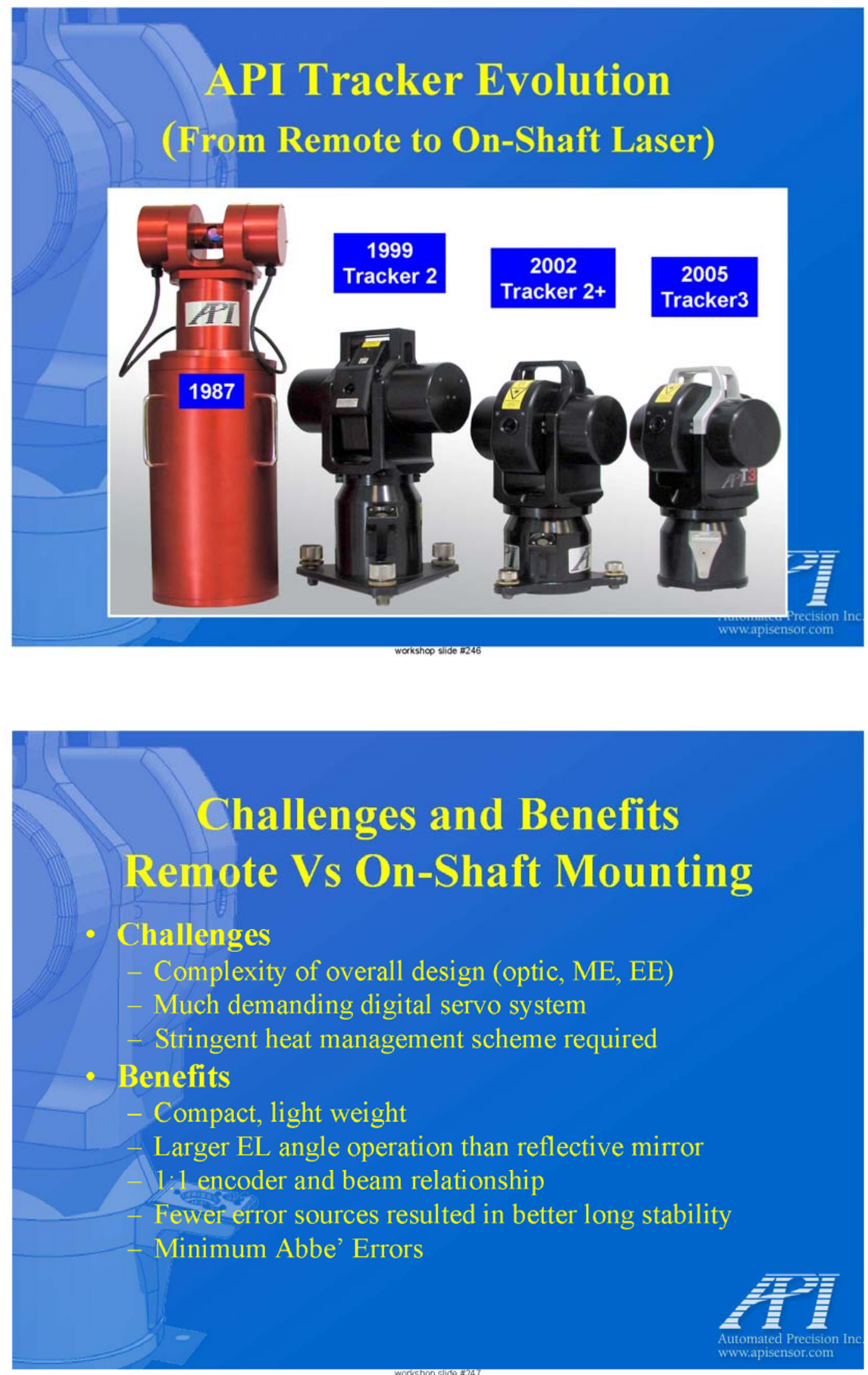

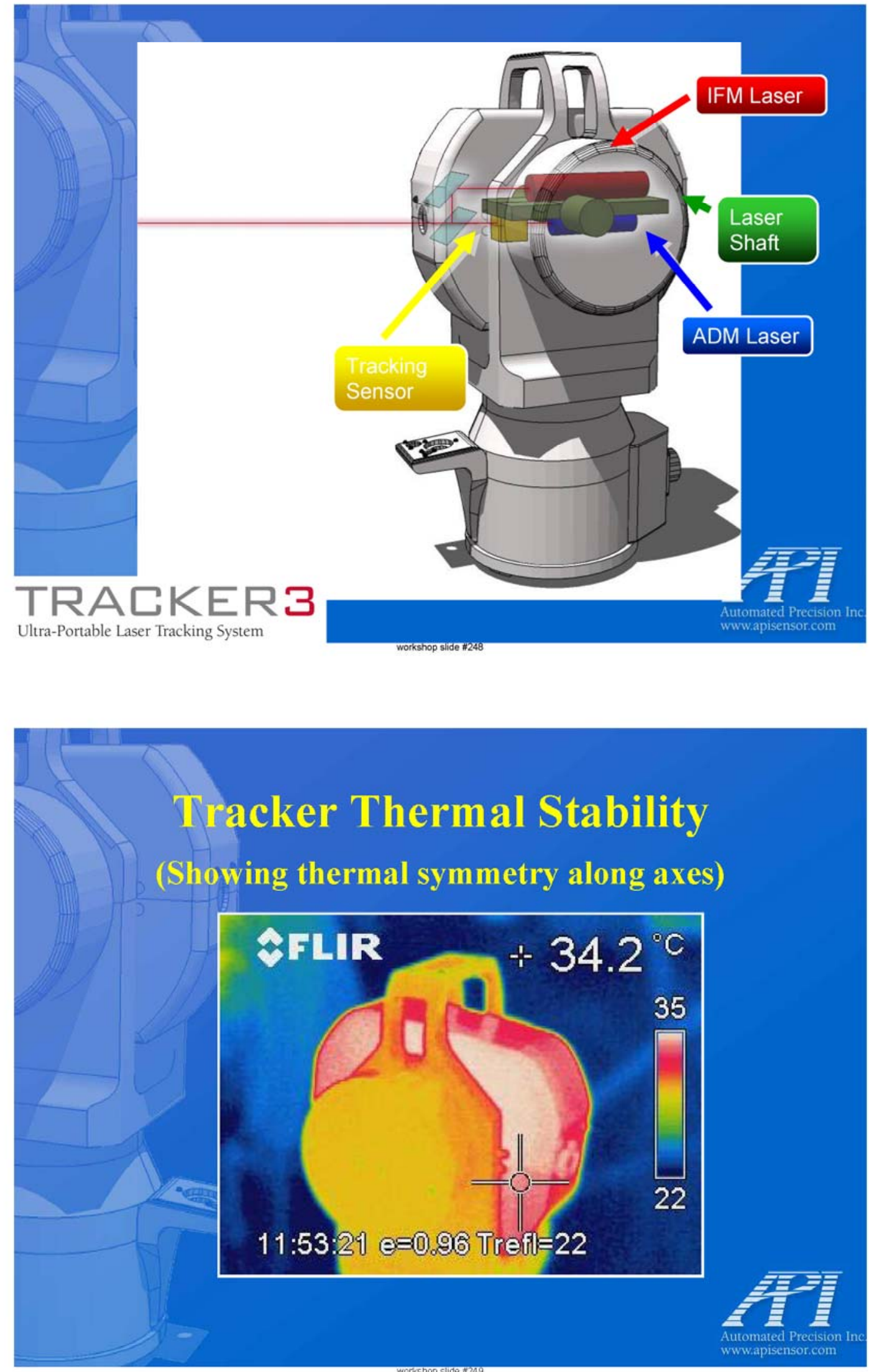


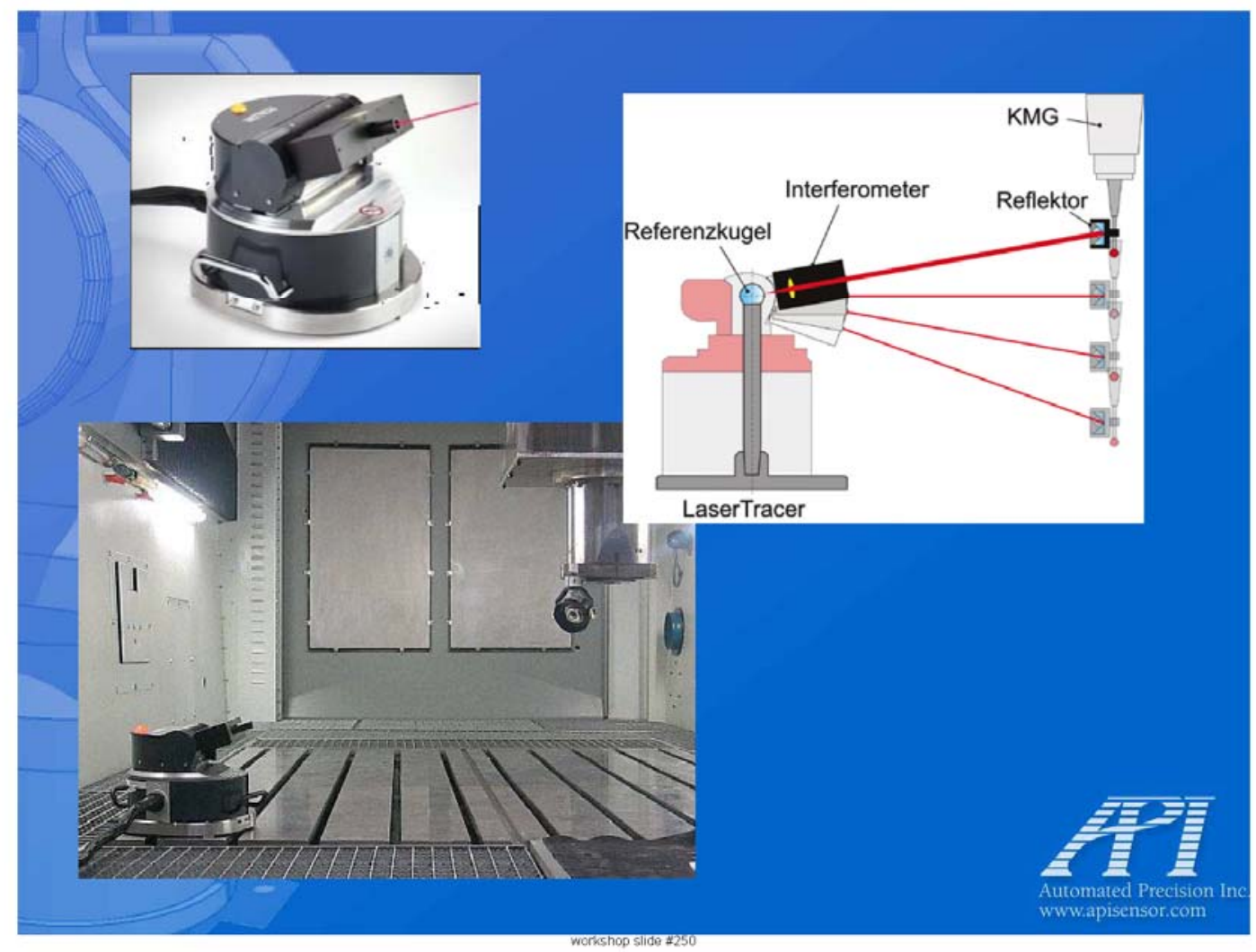

\section{Tracker Traceability}

New ASME B89.4.19 Tracker Calibration Standards calls for measurements against a traceable length artifact
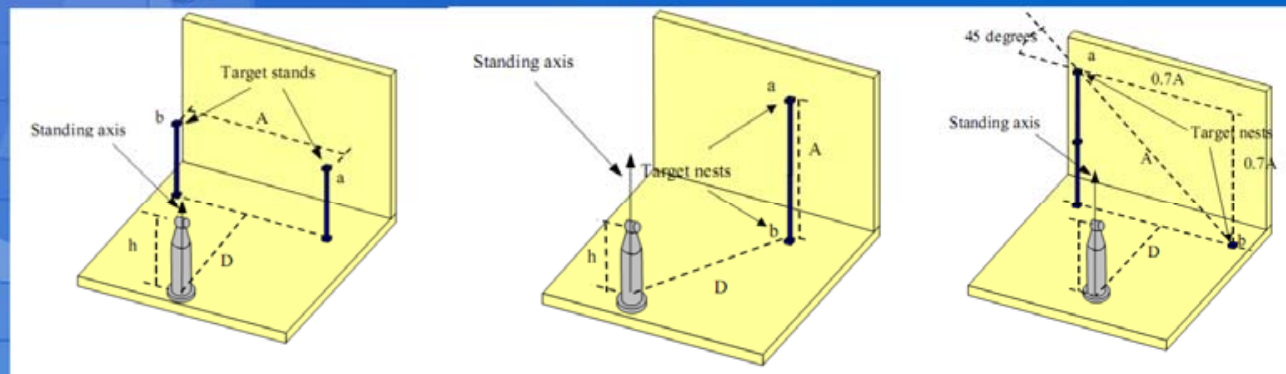

Figure 6.3.3. Measurement geometry for horizontal length measurement system tests

Figure 6.3.6. Measurement geometry for left diagonal length measurement 


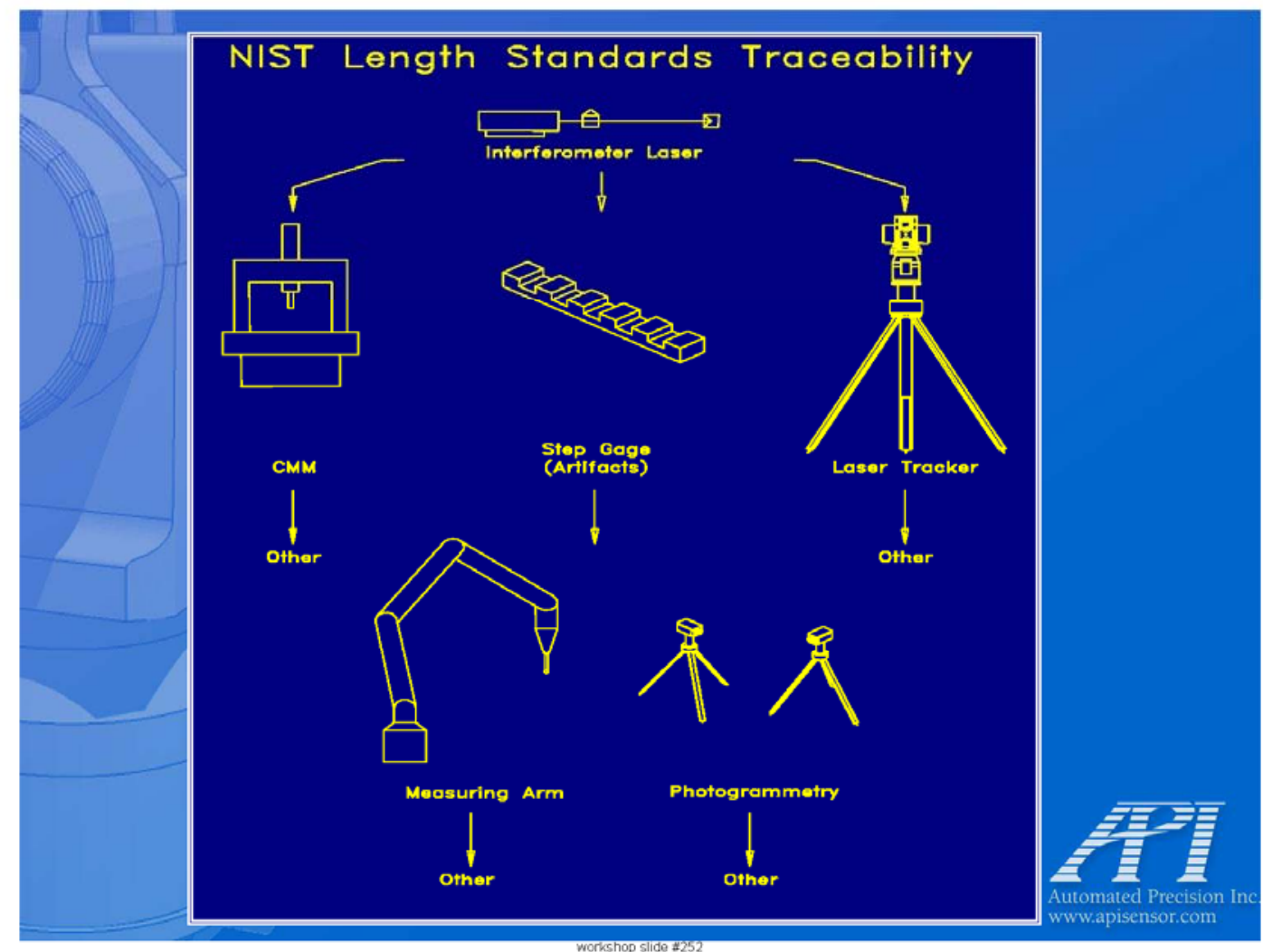

\section{USMN Vs ASME B89}

- Laser interferometer is the internationally accepted length standard

- Laser tracker is the only portable 3-D measuring instrument with built-in length standard

- One of the major reasons for the popularity of laser tracker!!

- USMN (Unified Spatial Metrology Network) utilizes the embedded interferometer for tracker self-certification

- Caution: ADM-only tracker is an exception 

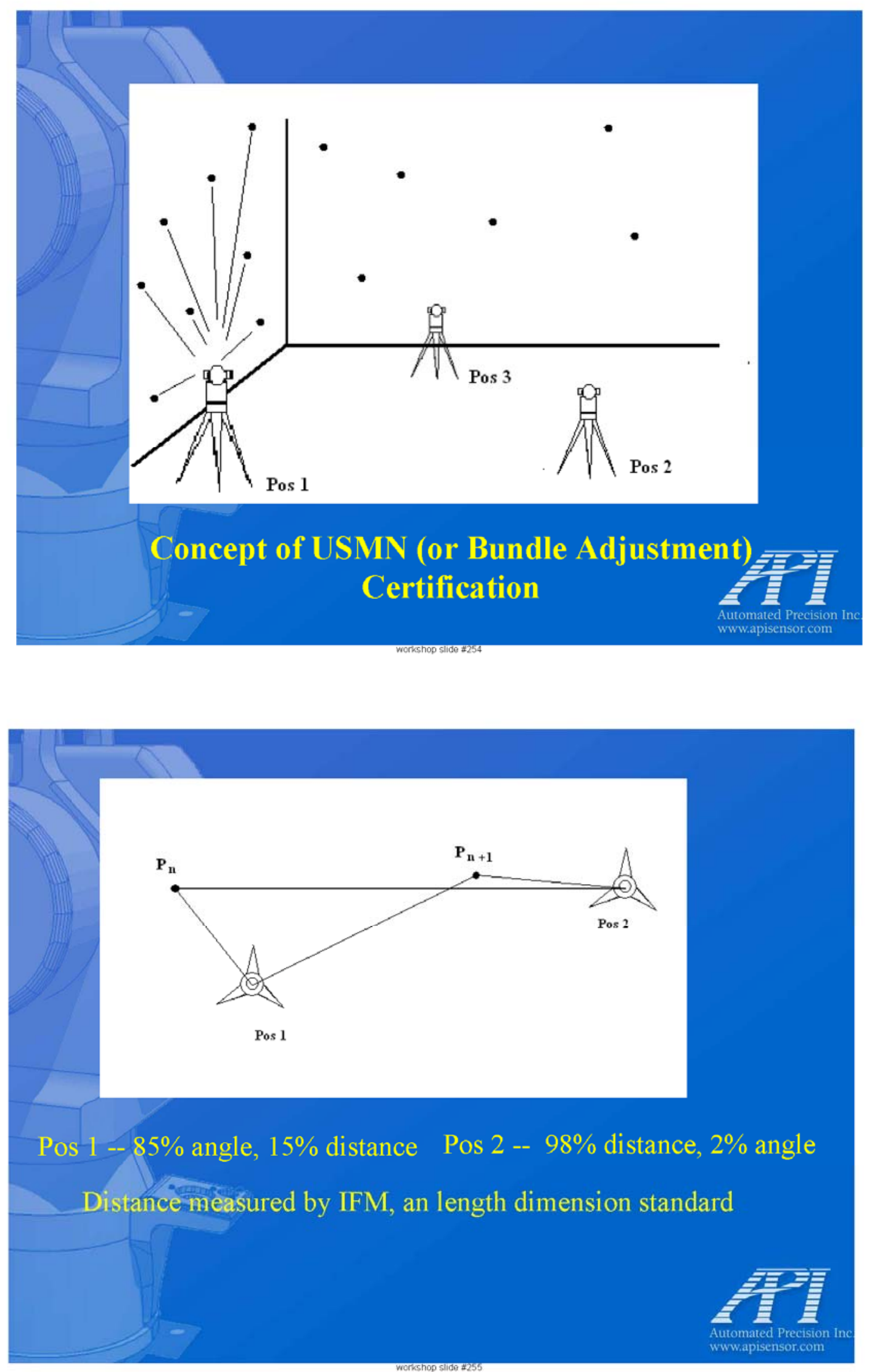


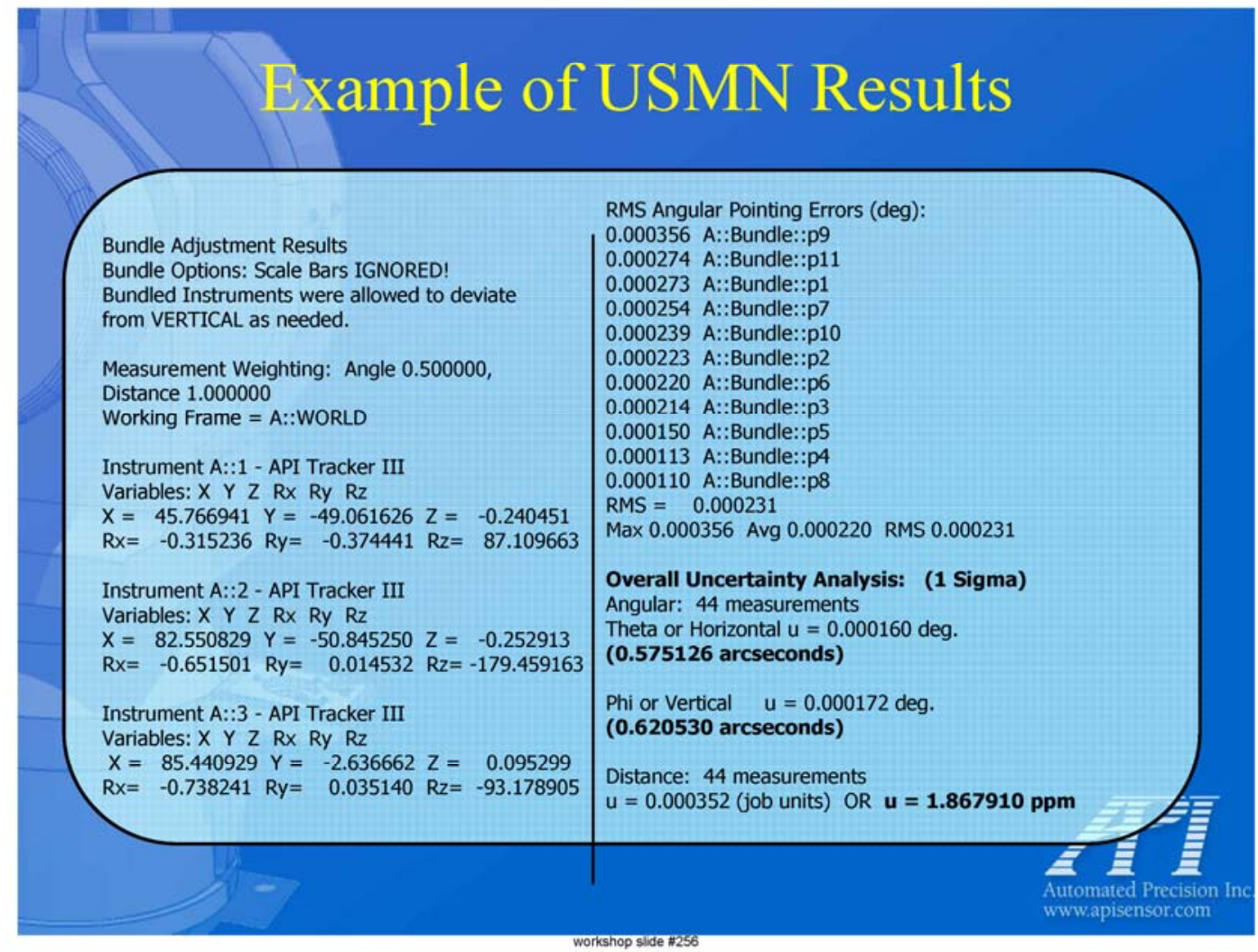

Workshop side 1235

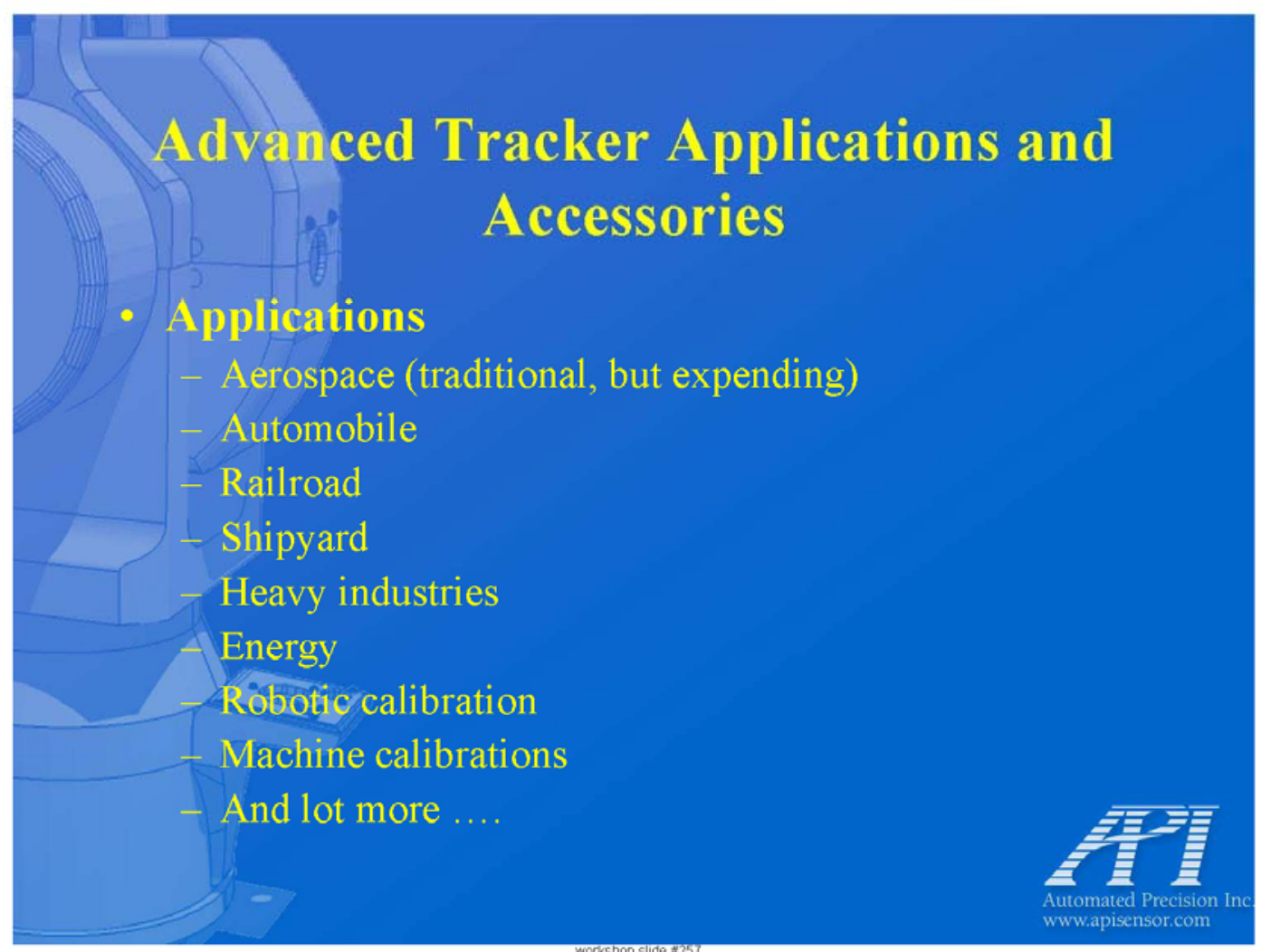

Workshop slide \$257 

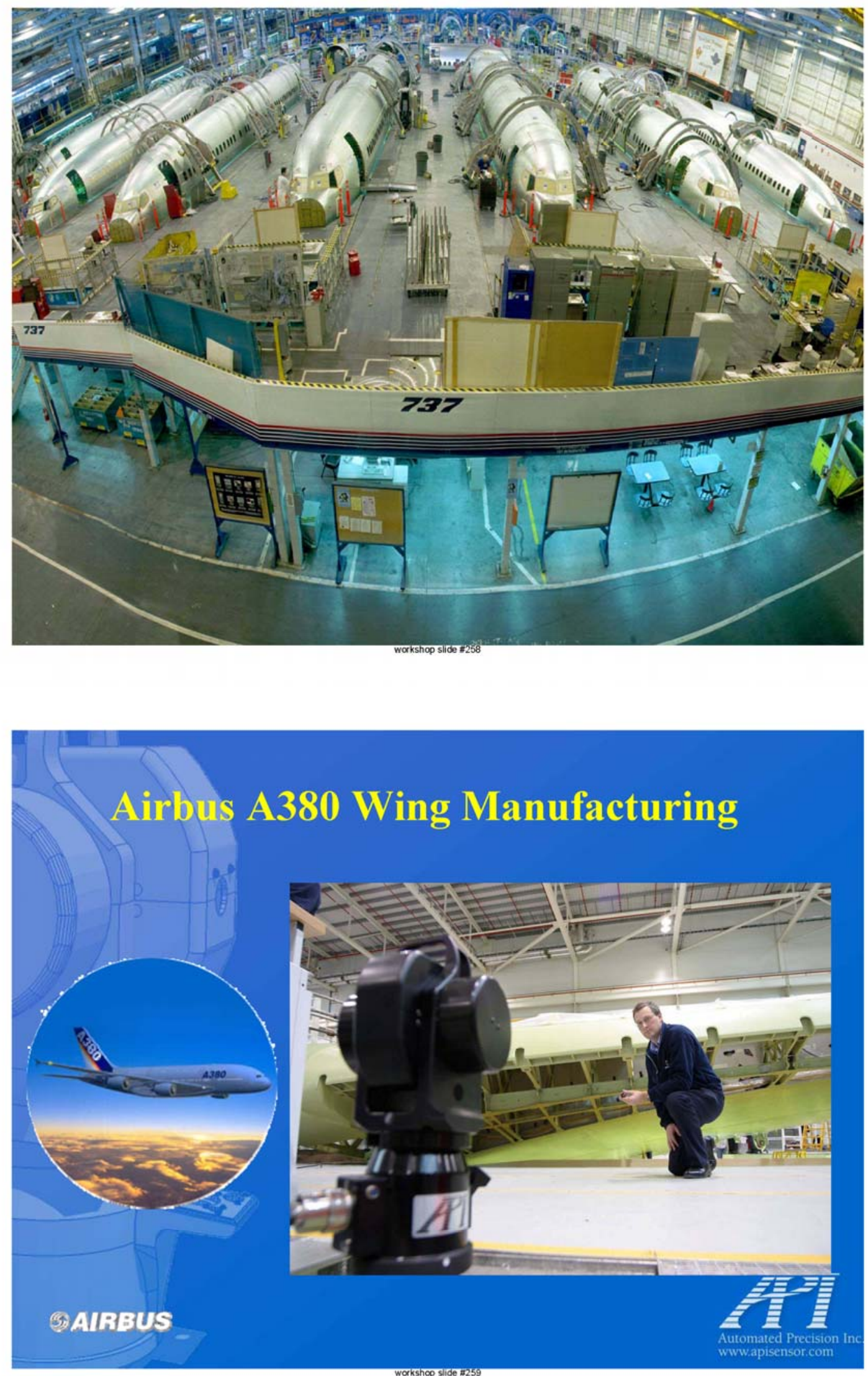

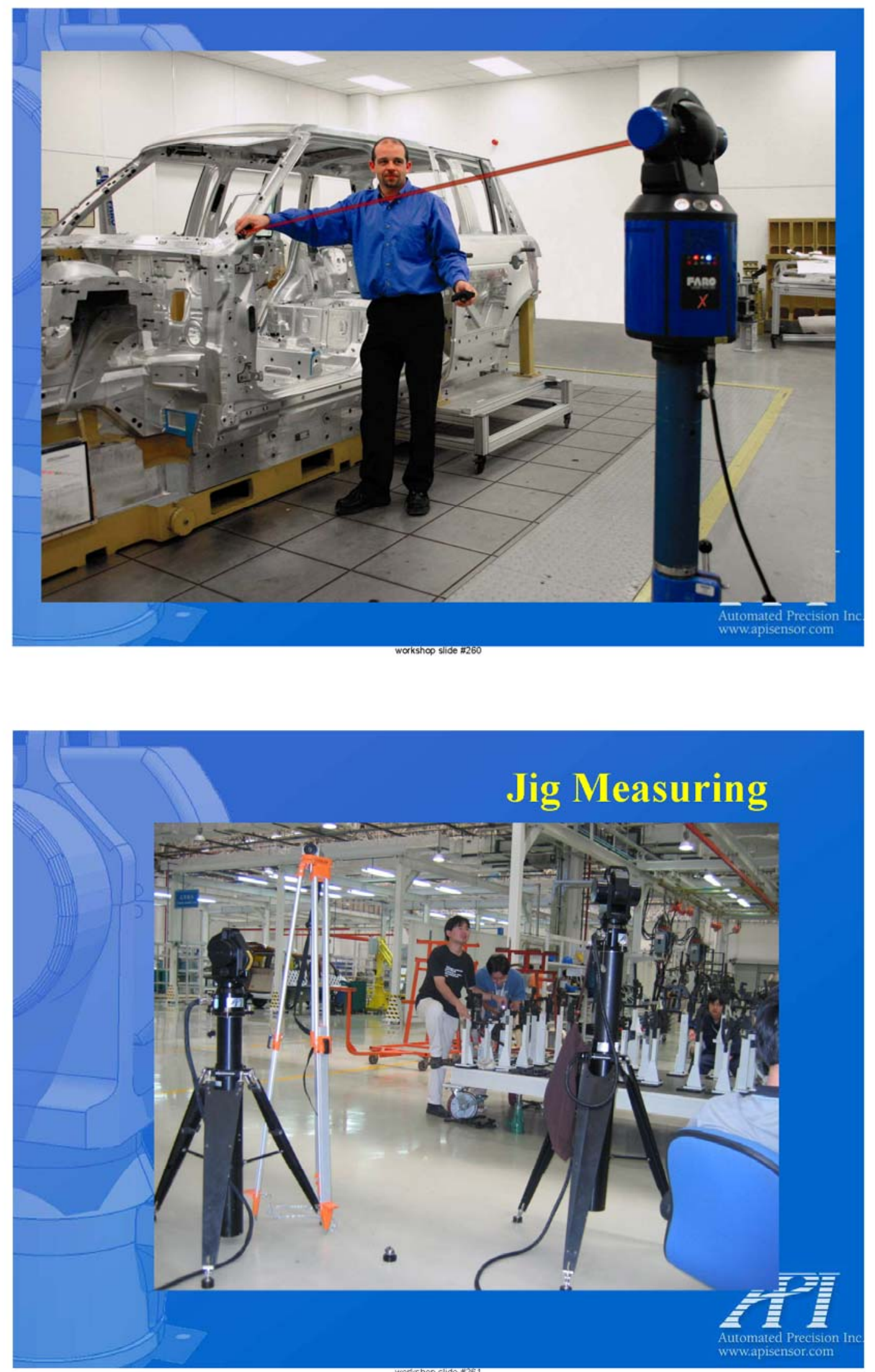


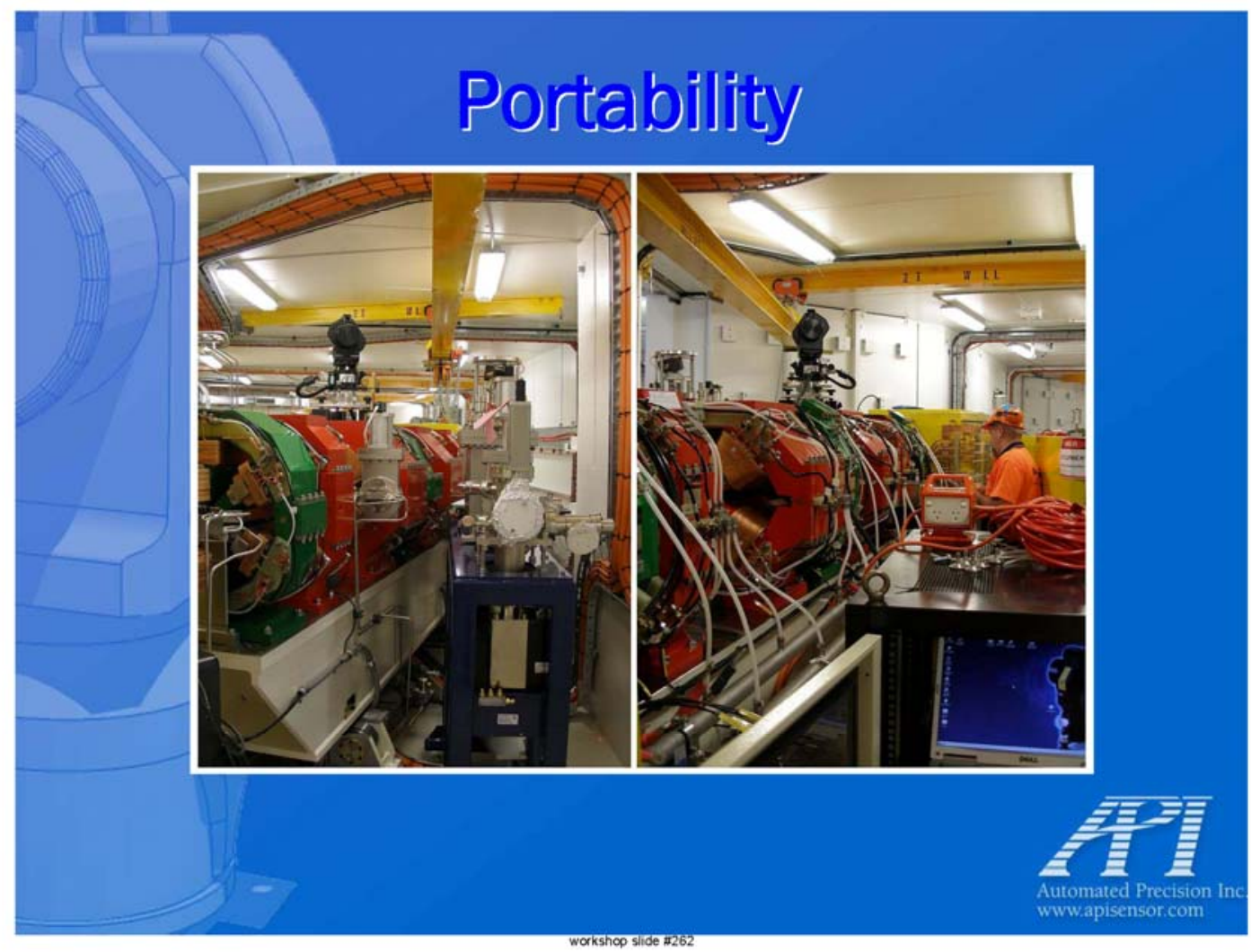

Workshop sirde "1262

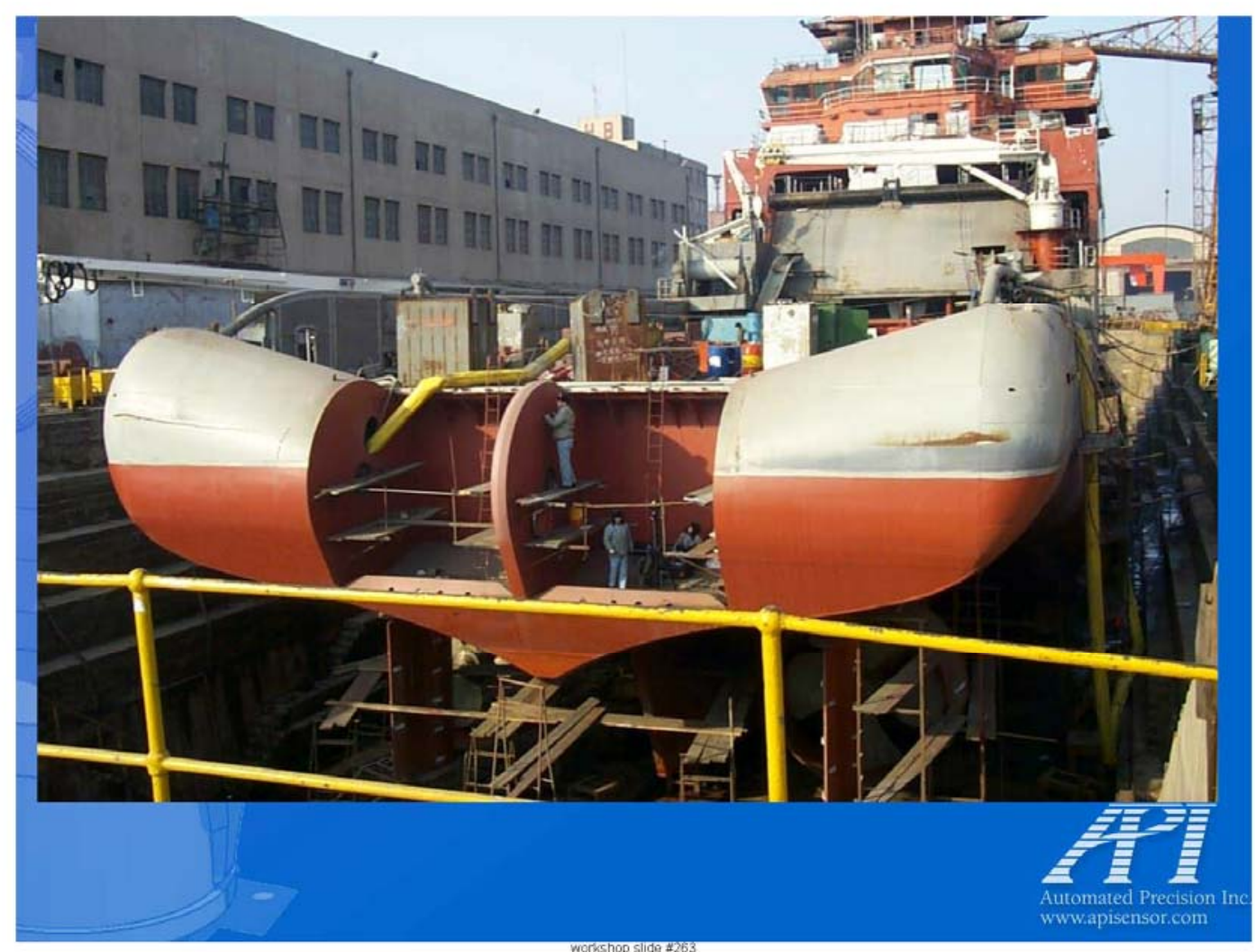



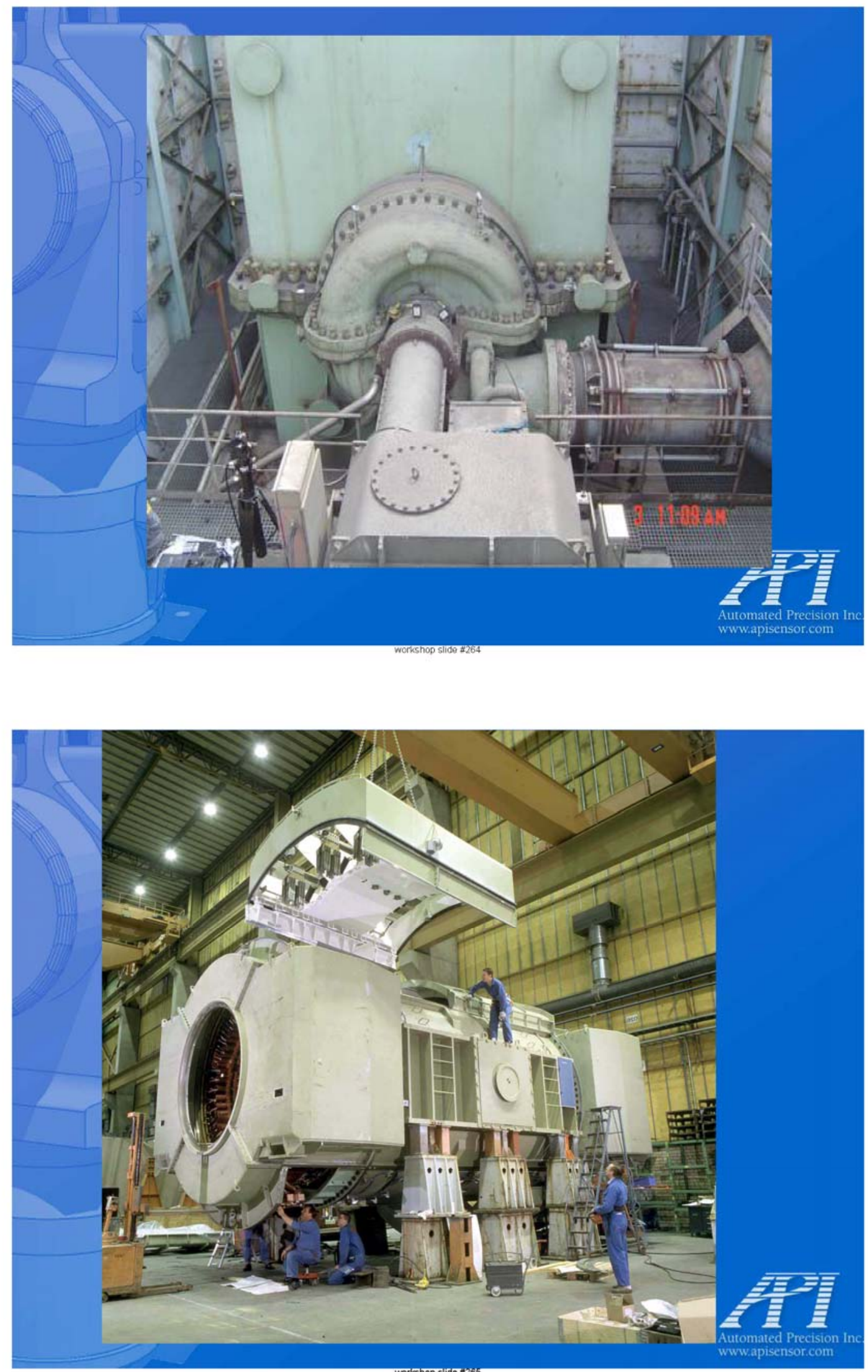


\section{Antenna Manufacturing and Installation}
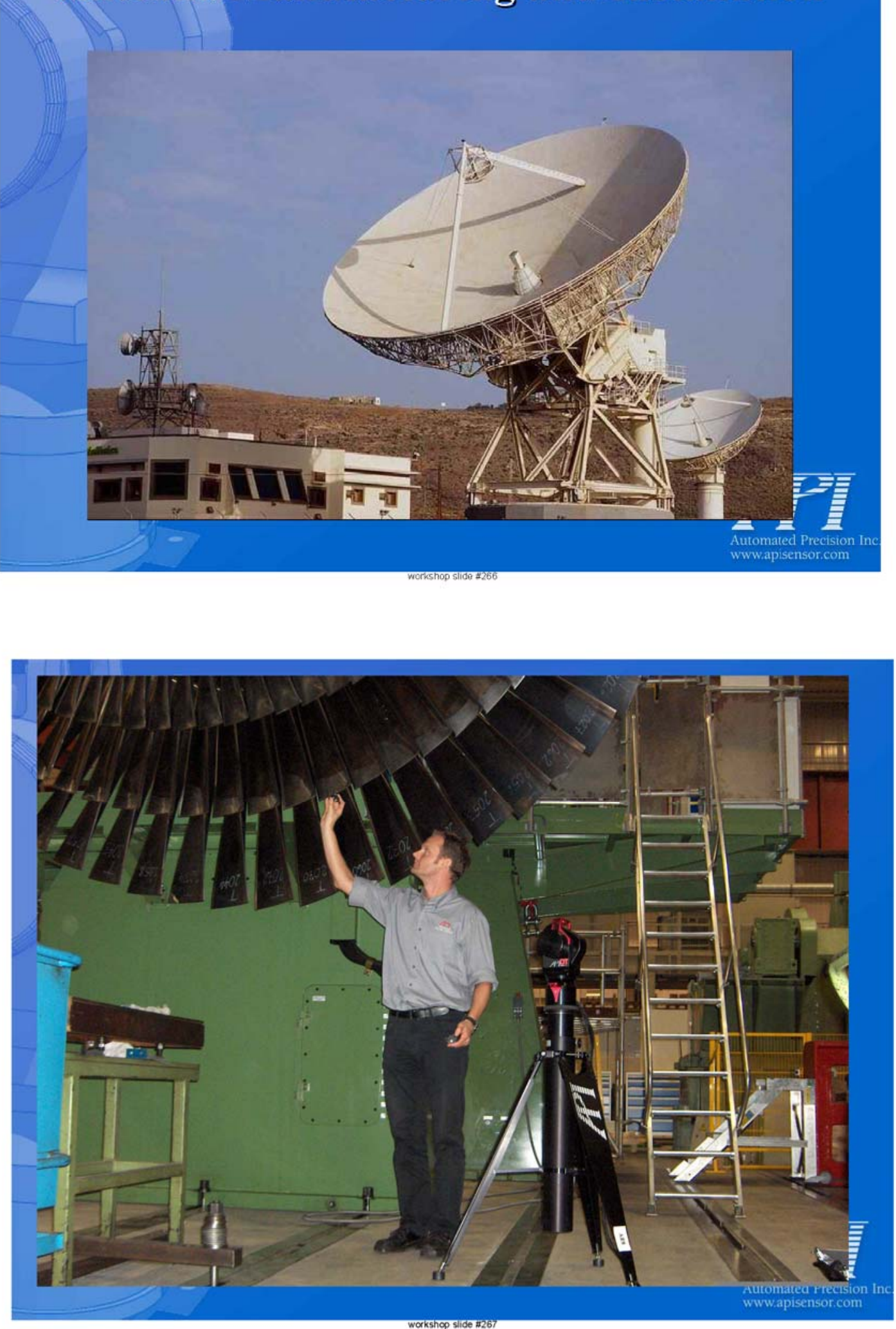

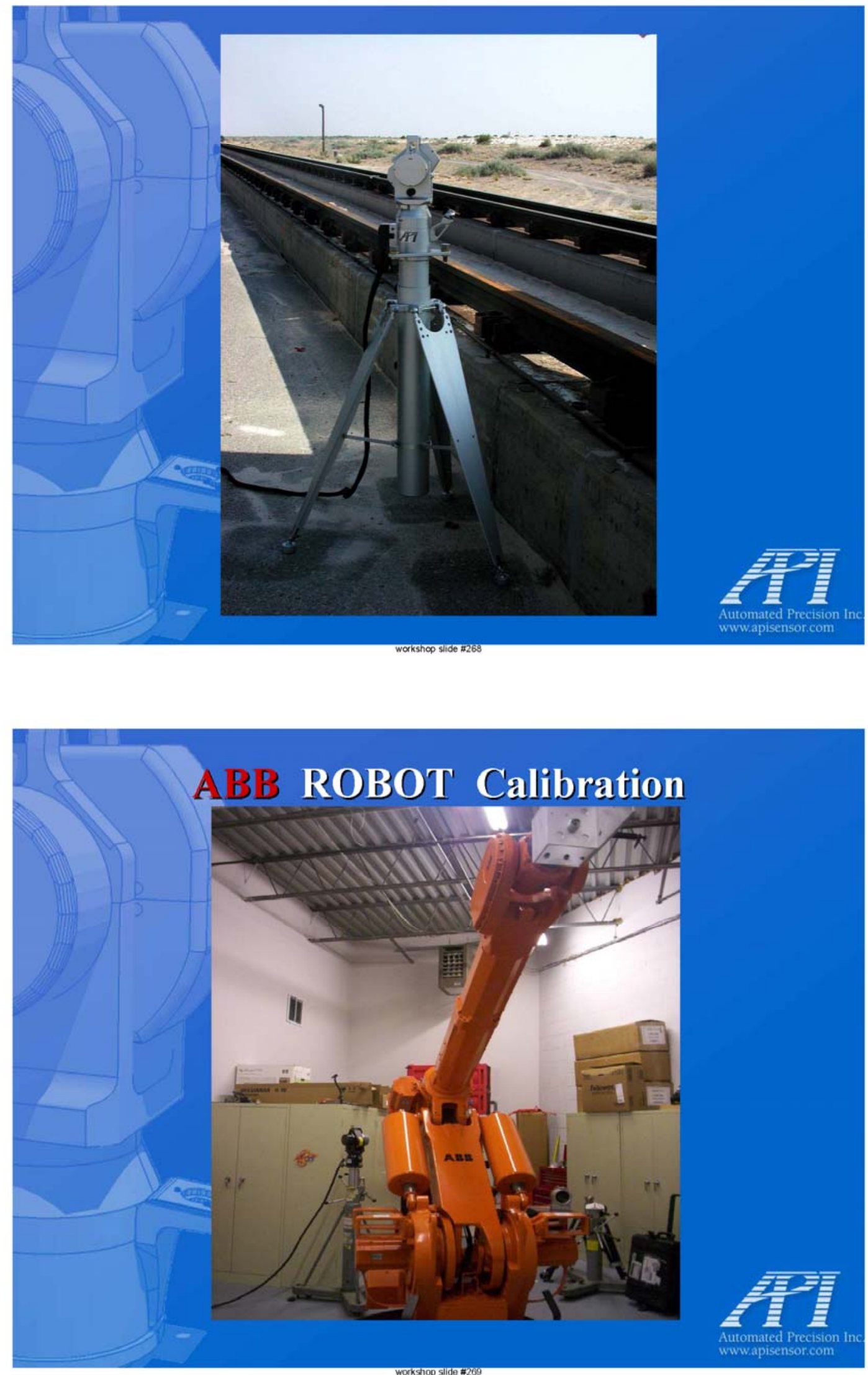


\section{New Development in Machine Tool Error Mapping with Laser Tracker}

- Traditional wisdom- tracker doesn't have enough precision

- Work at Boeing St. Louis successfully demonstrates it is possible

- Phil Freeman and Sam Easley successfully mapped 11 shop floor machines in various Boeing plants providing 3 to 4 times improvements of volumetric accuracy

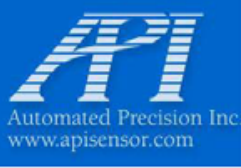

rikshop silde $\$ 270$

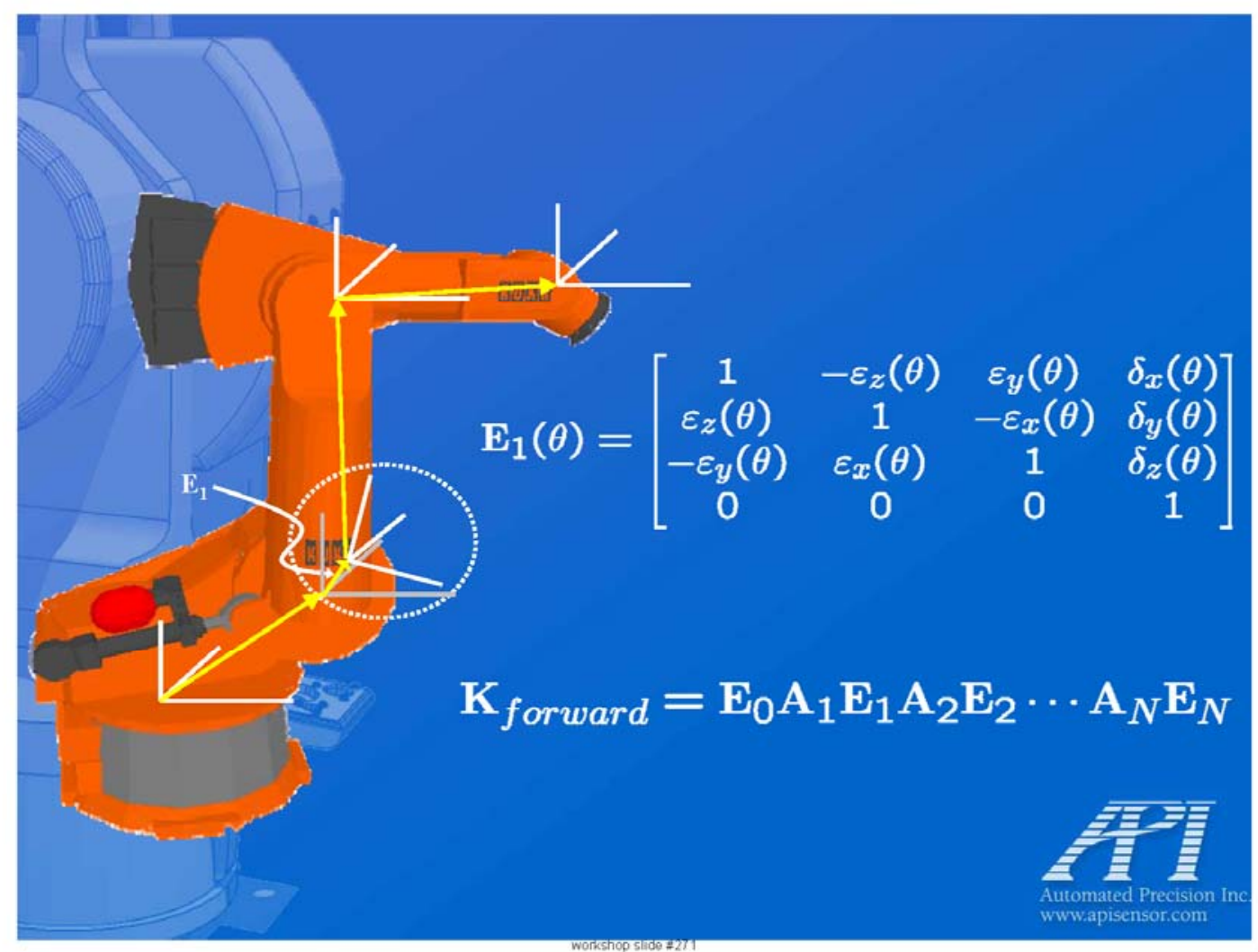




\section{Motion Errors}
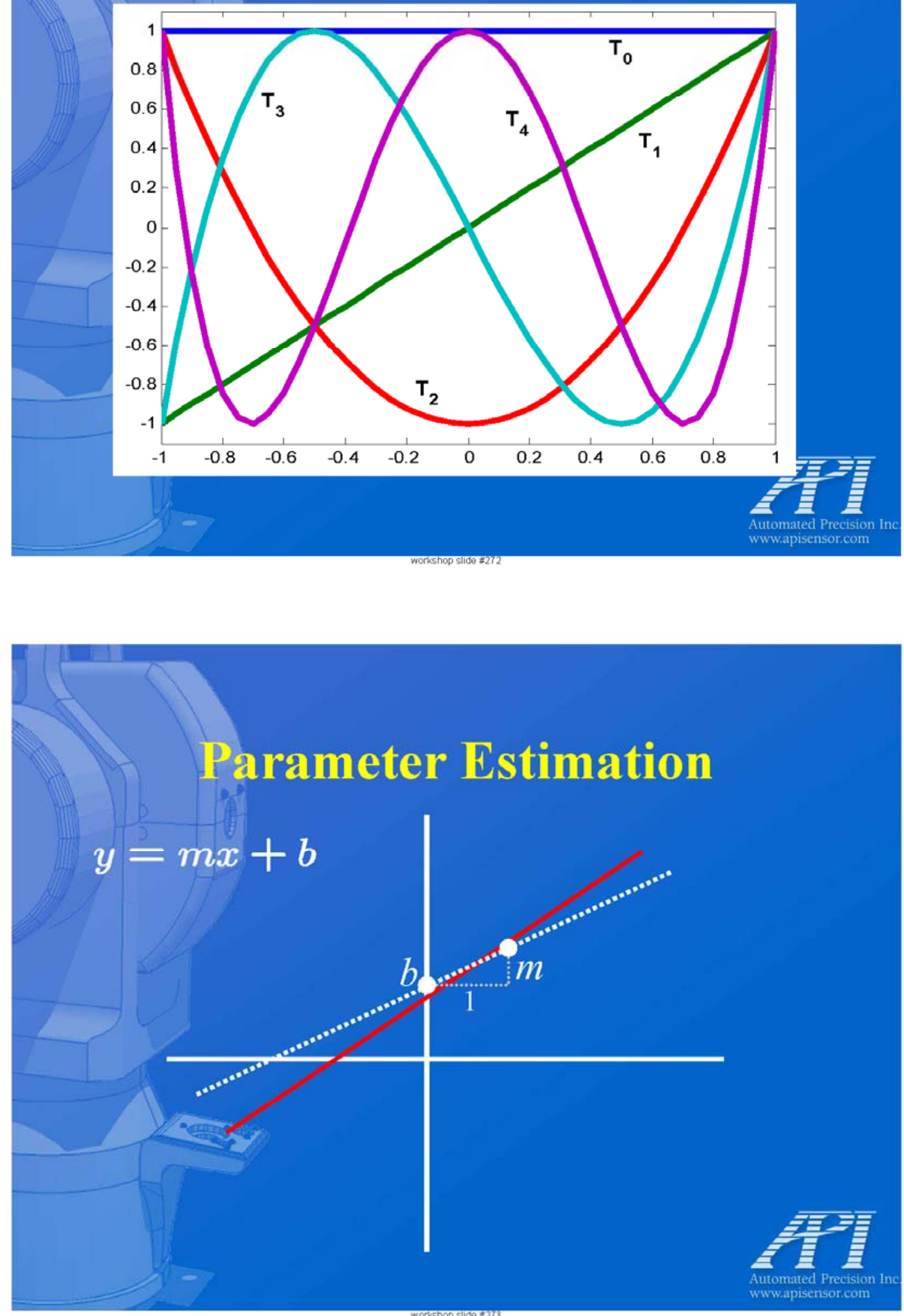


\section{Parameter Estimation}

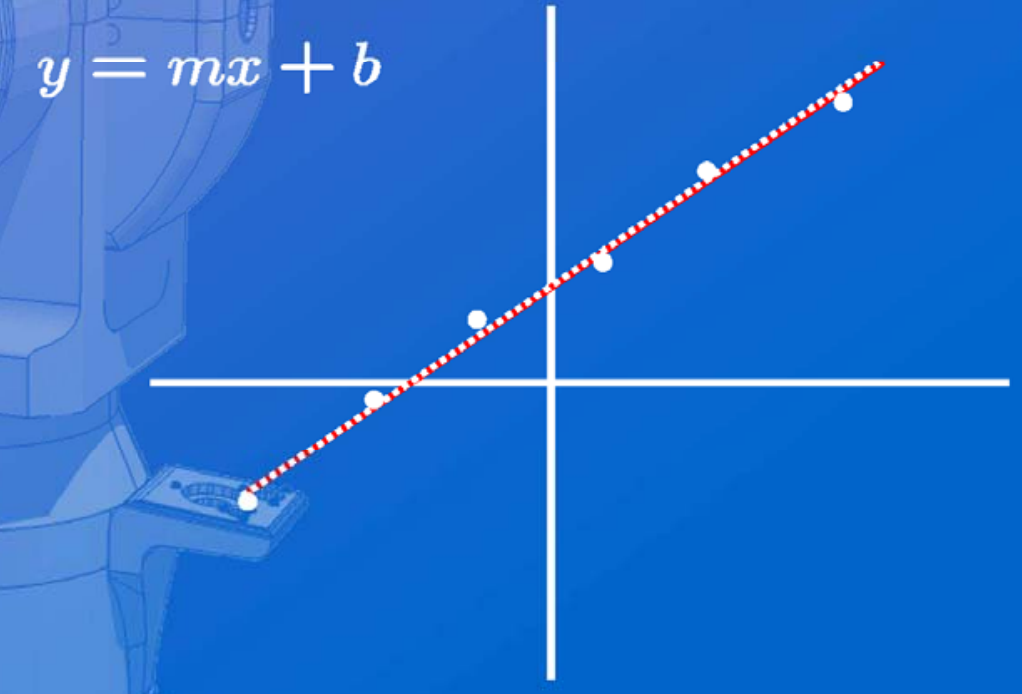

AI

Workehop ज्ञात $22 / 4$

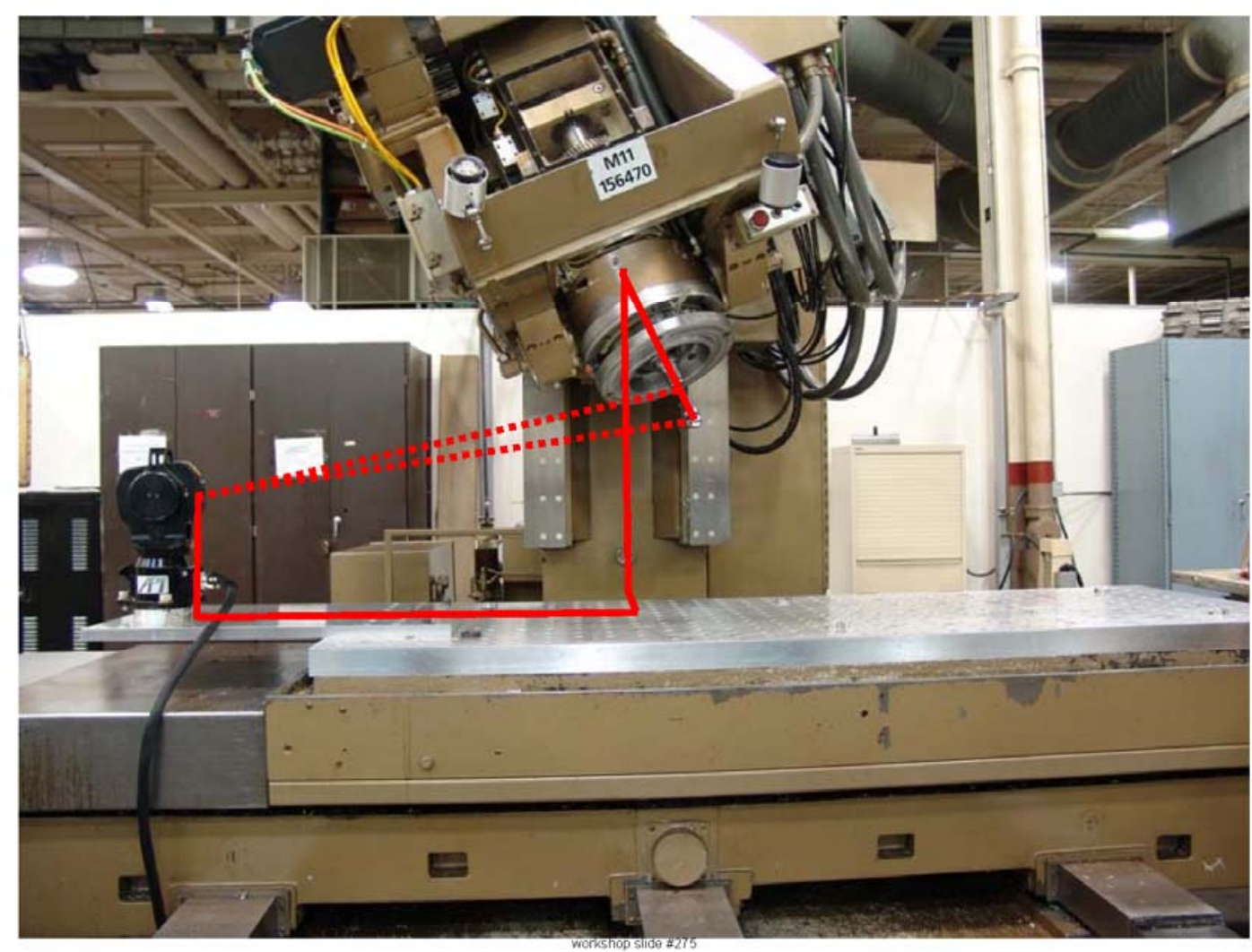




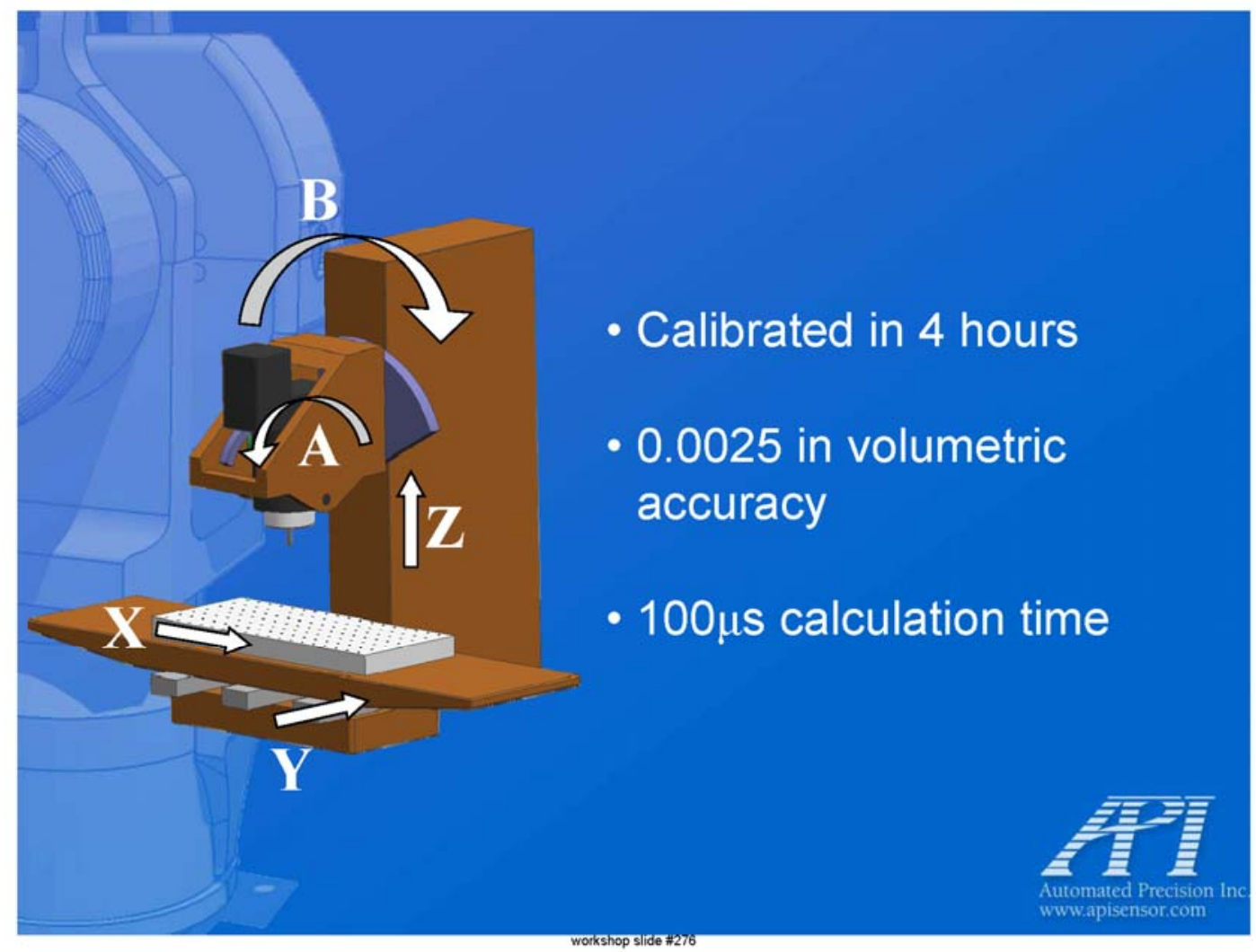

Every Measurement Improves the Estimate of Every Parameter
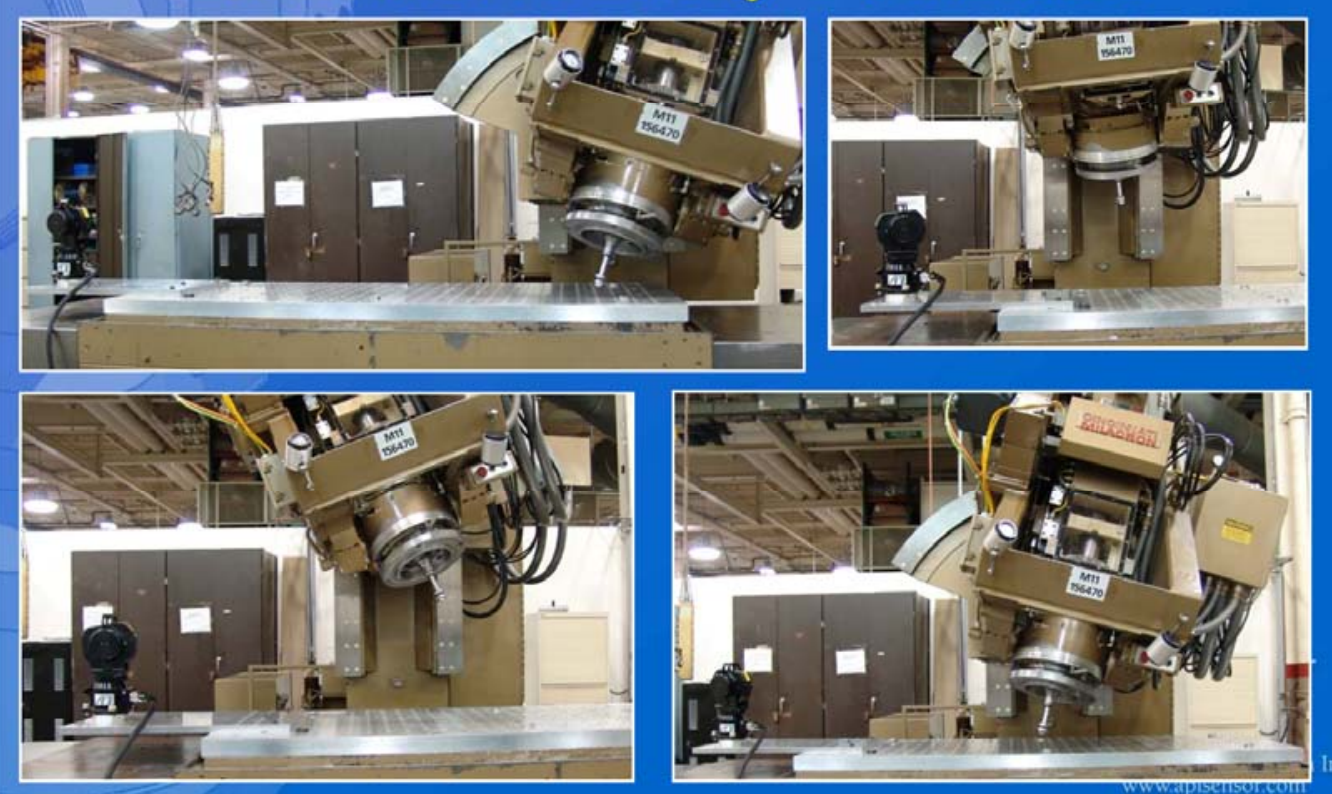

workshop stde $\$ 2$ ?7 


\section{Before and After Accuracy}
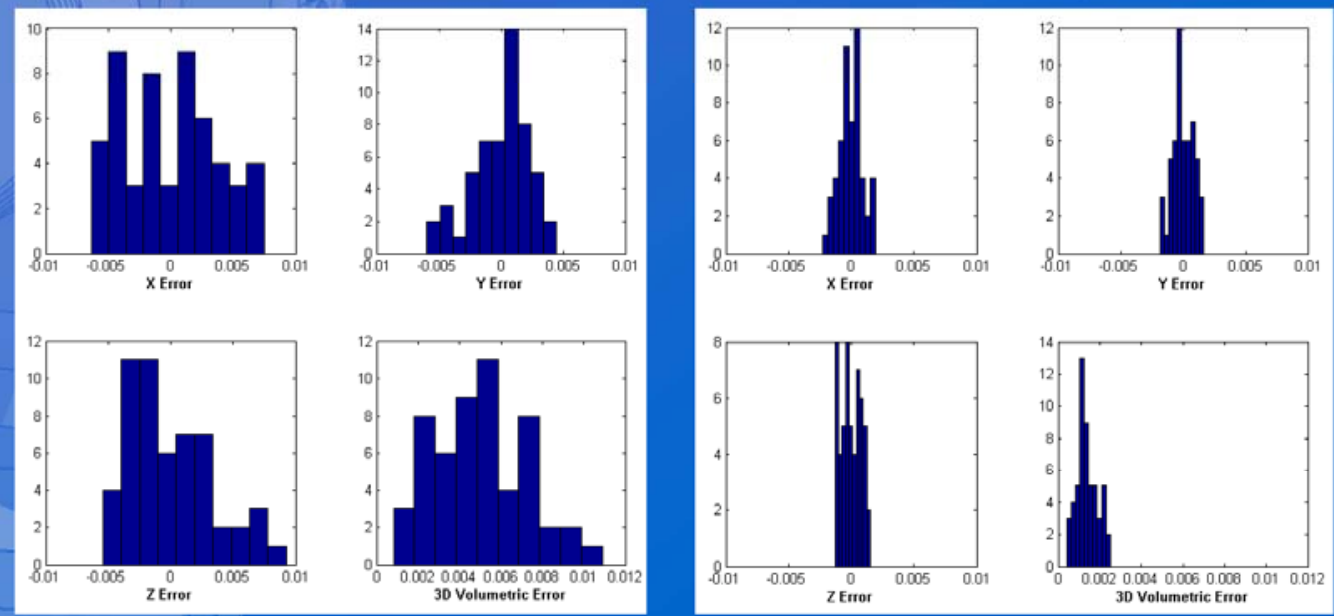

Before
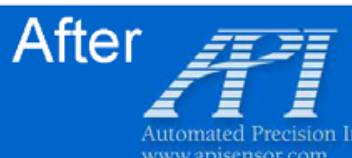

\section{Test Part Results}
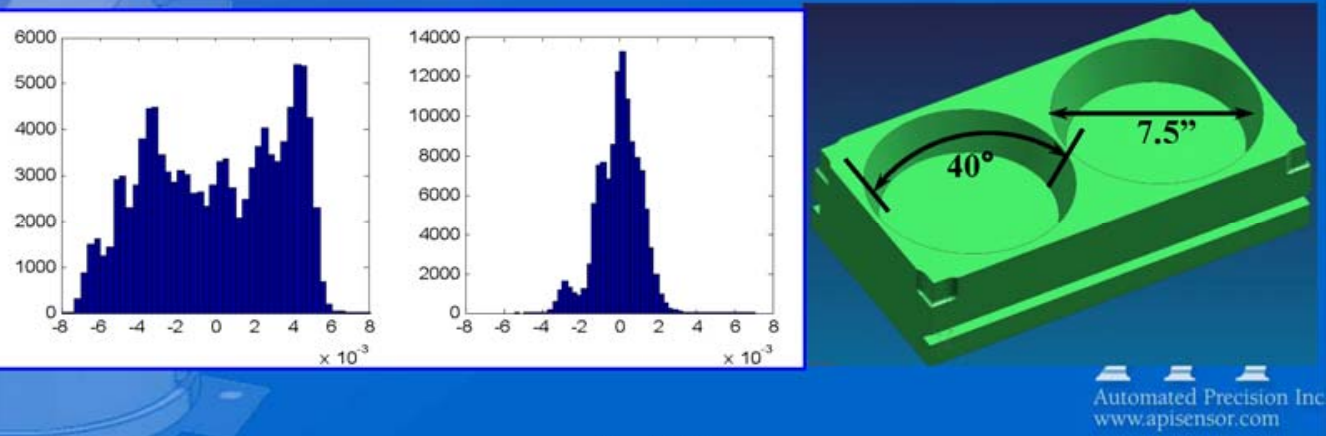

workshop sirde 1279 


\section{Advanced Tracker Accessories}

Advanced accessories give tracker more

versatilities in dealing with difference

measuring challenges

- Hidden points

- Surface scanning

- Non-contact

- High data-rate point-cloud

- 6-D measurements

- Programmable automation

workshop slide $\$ 280$
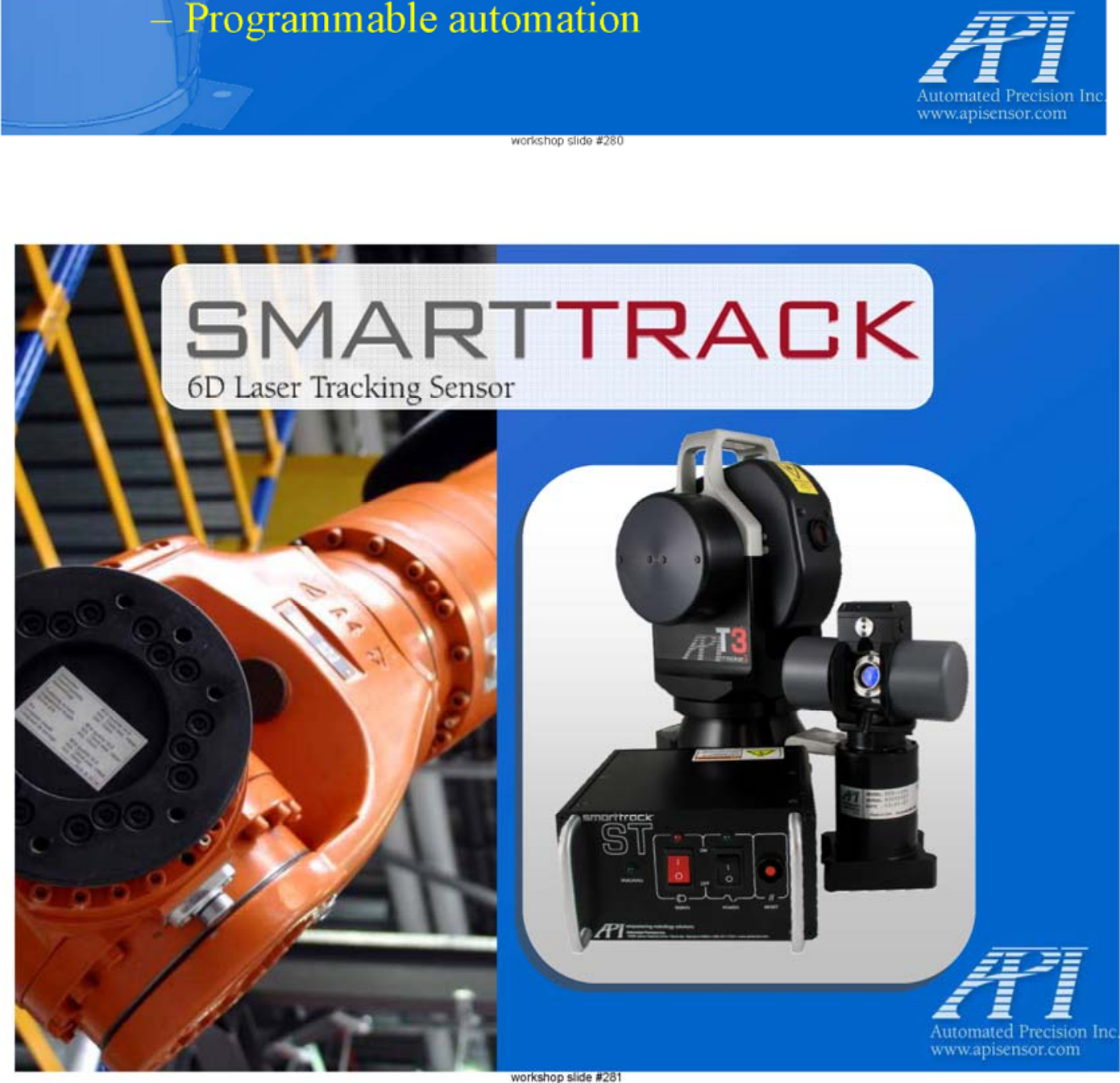

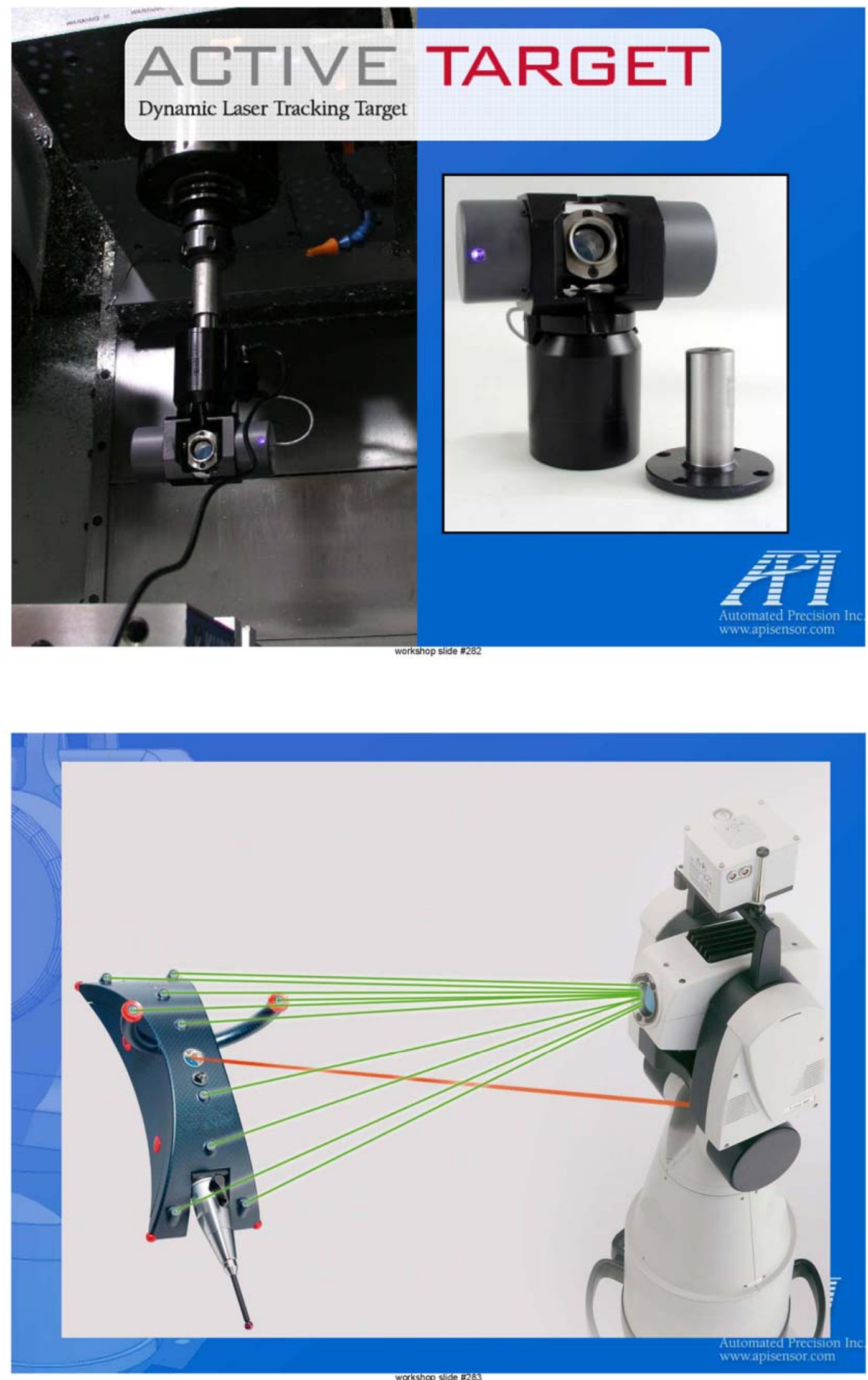

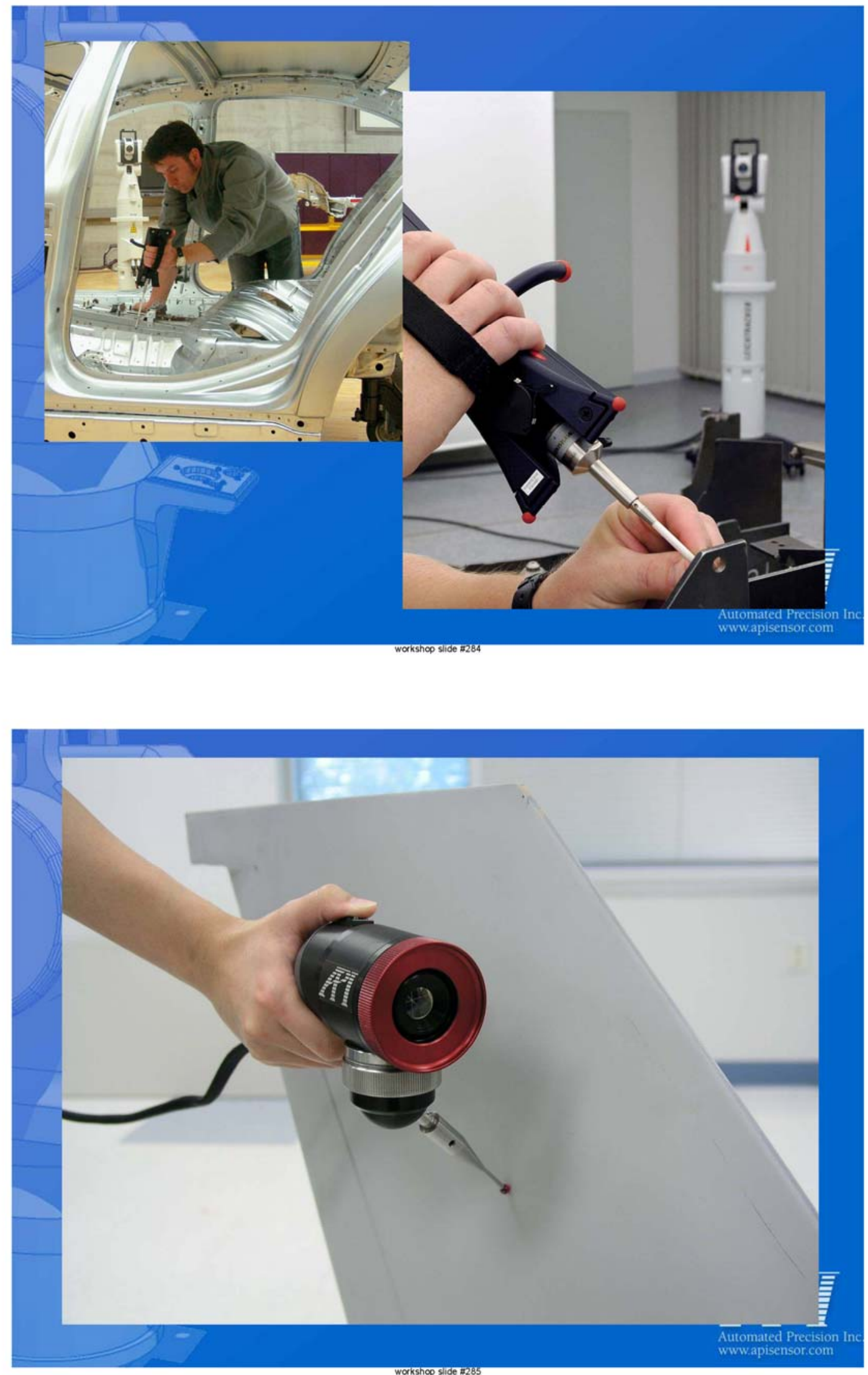


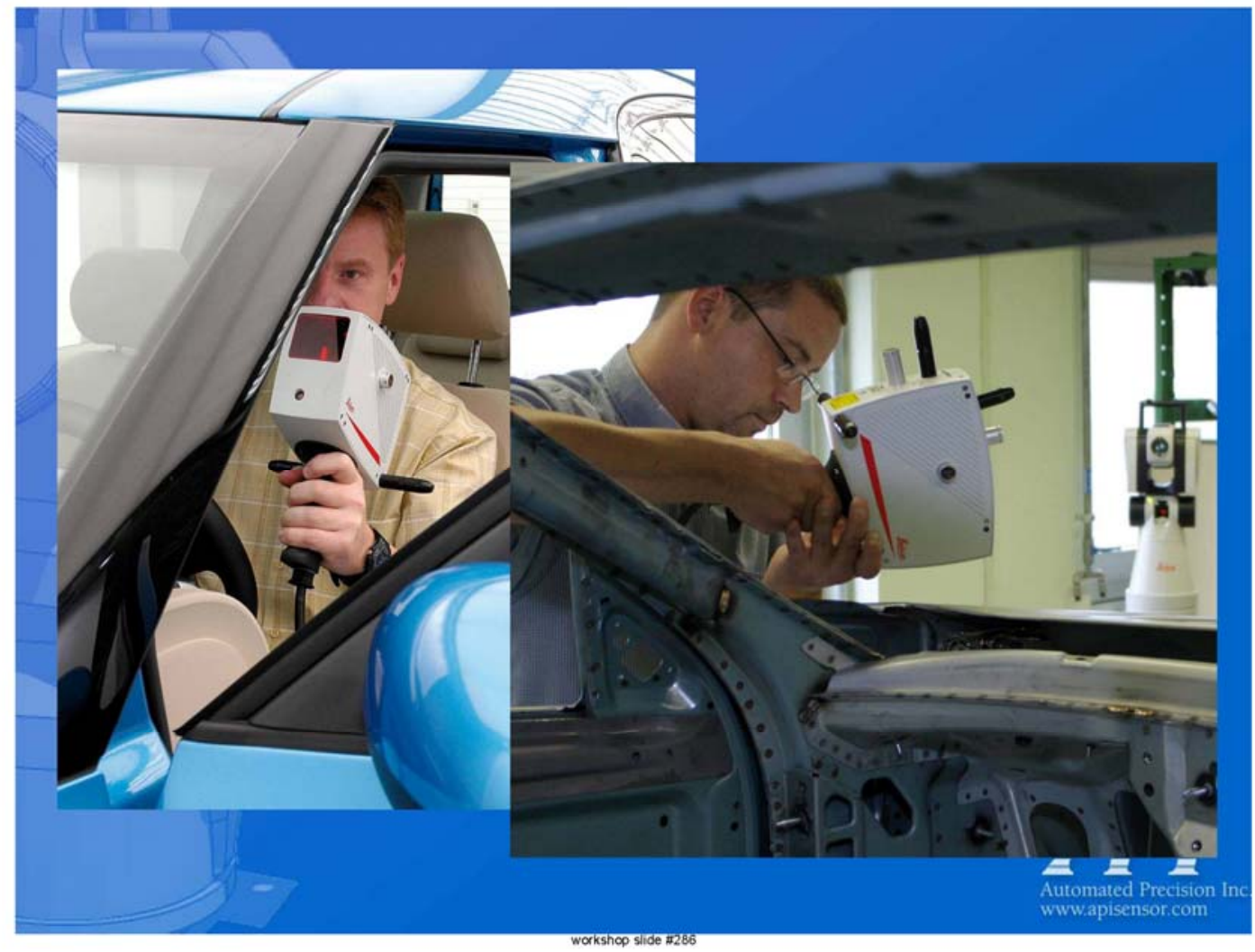

orkshop sirde स286

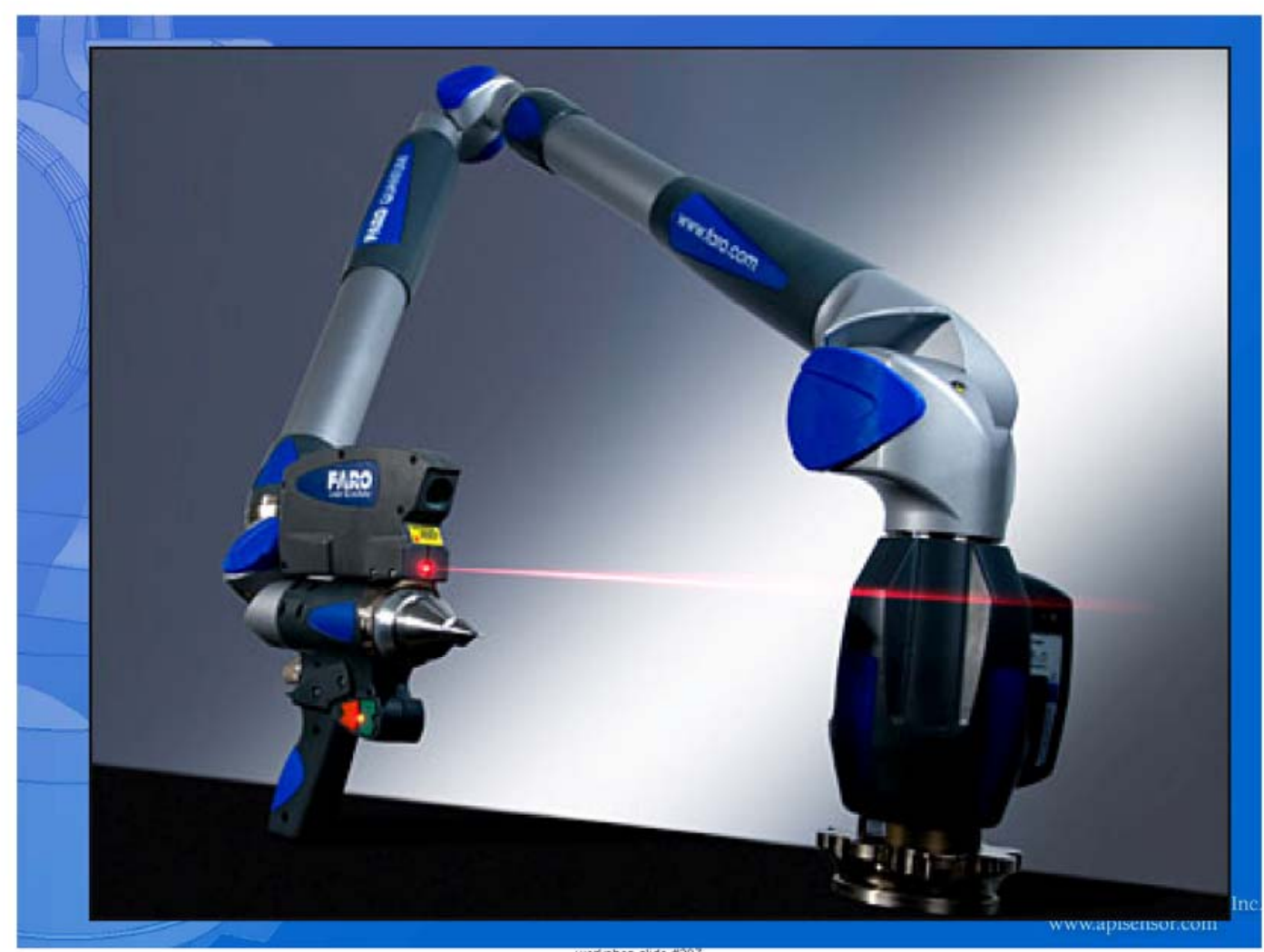




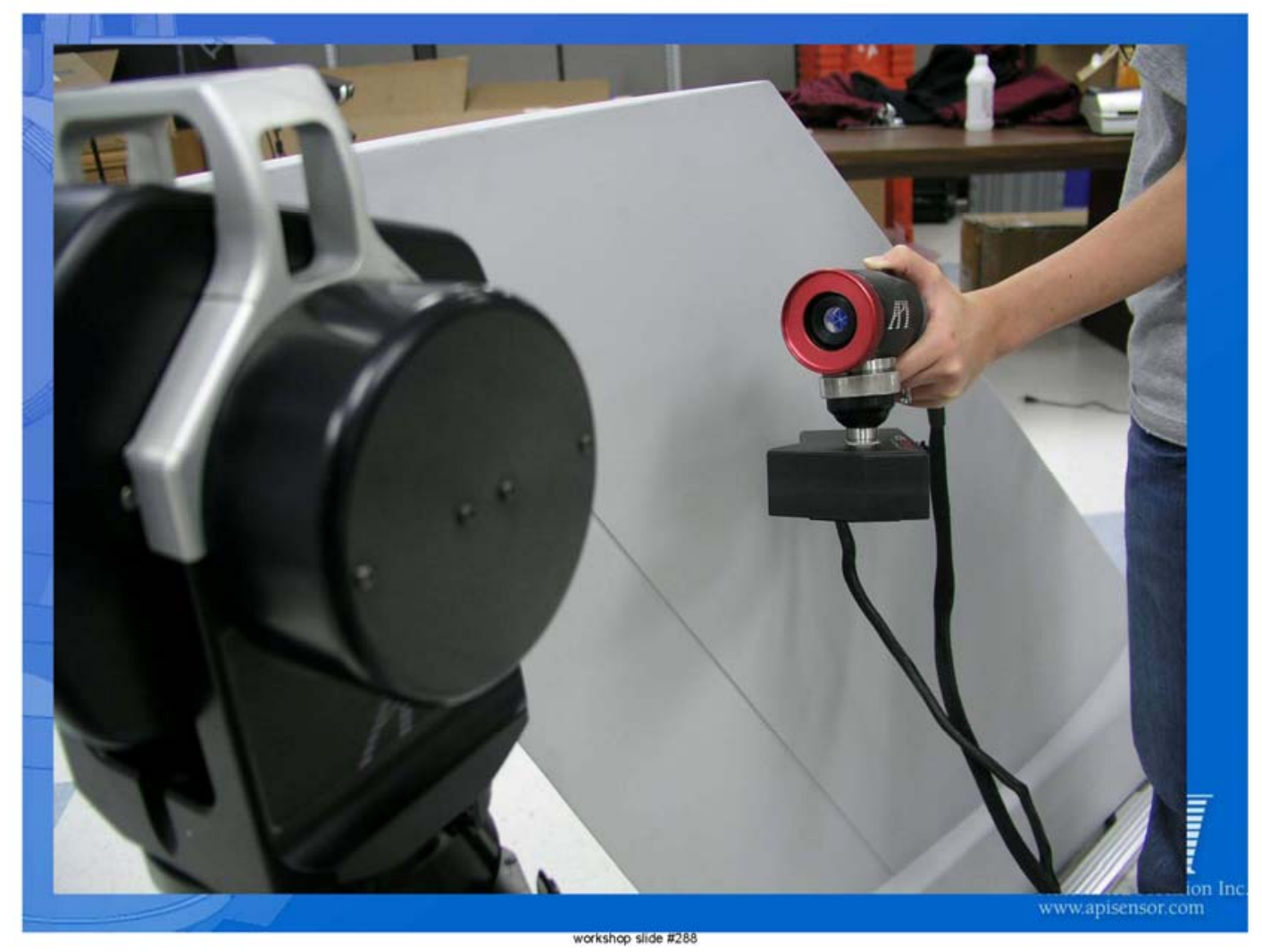

\section{Trends of Laser Tracker Technology and Applications}

- Applications from aerospace to automobiles, antenna, shipyard, machine tools, heavy industries, ...

- Smaller, lighter, lower cost, more precise, longer range, field certifiable, more features

- Advanced accessories like hand-held probes (contact or non-contact), multiple-degree of freedom tracking, integration with arms, cameras, electronic levels, photogrammetry, optical surface scanners

- New market continues to grow as applications expand

- Becomes a major threat to CMM and theodolite markets 